\title{
Acylative Dynamic Kinetic Resolution of Secondary Alcohols: Tandem Catalysis by HyperBTM and Bäckvall's Ruthenium Complex
}

\author{
Artis Kinens,${ }^{\dagger \S}$ Simonas Balkaitis, ${ }^{\dagger \S}$ Omar K. Ahmad, ${ }^{\ddagger}$ David W. Piotrowski ${ }^{\dagger *}$ and Edgars Suna ${ }^{\dagger \S *}$ \\ ${ }^{\dagger}$ Latvian Institute of Organic Synthesis, Aizkraukles 21, LV-1006, Riga, Latvia \\ $\S$ University of Latvia, Department of Chemistry, Jelgavas 1, LV-1004, Riga, Latvia \\ *Worldwide Medicinal Chemistry, Pfizer, Inc., Eastern Point Road, Groton, CT 06340, USA \\ david.w.piotrowski@pfizer.com,edgars@osi.lv
}

\section{Contents}

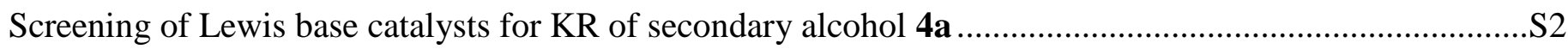



Screening of transition metal catalysts for the racemization of secondary alcohol $4 \mathbf{a}$................................S4

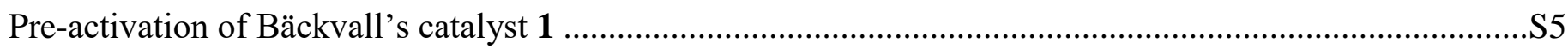

Screening of the compatibility of racemization conditions and acylating reagents.....................................S6

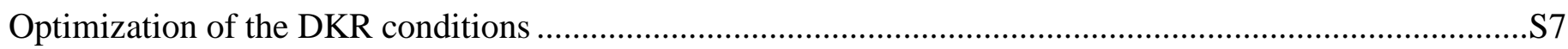

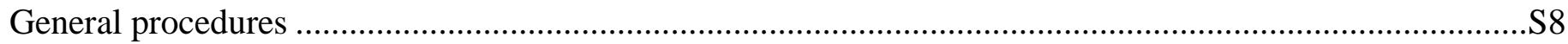

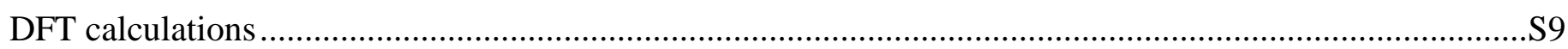

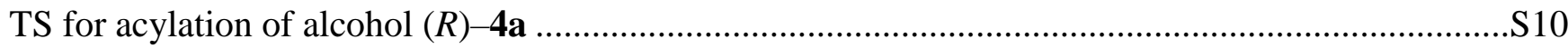

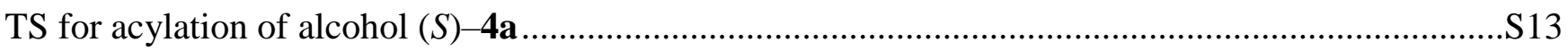

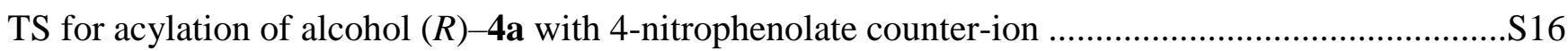

TS for acylation of alcohol $(S)-\mathbf{4 a}$ with 4-nitrophenolate counter-ion..............................................S19

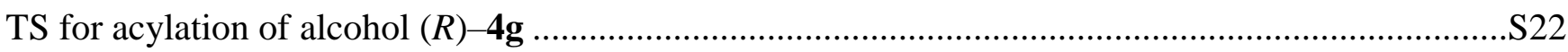

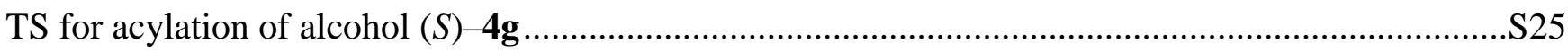

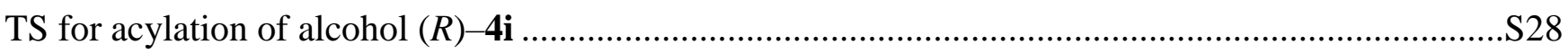

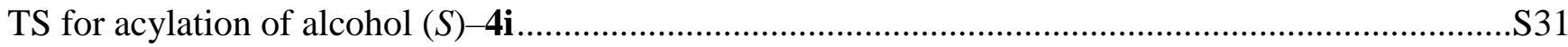

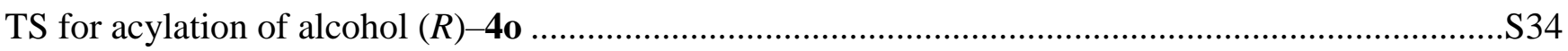

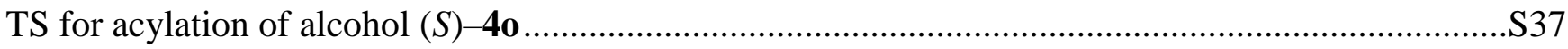

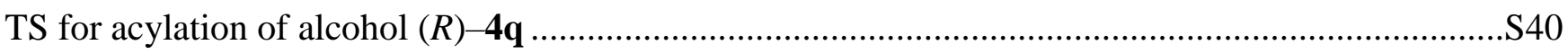

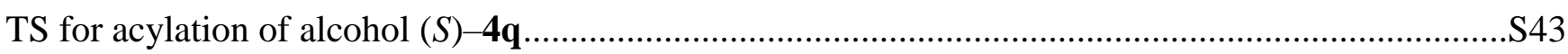

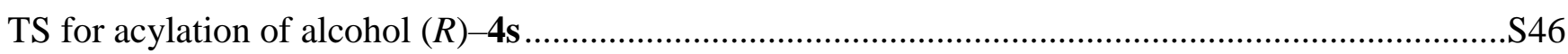

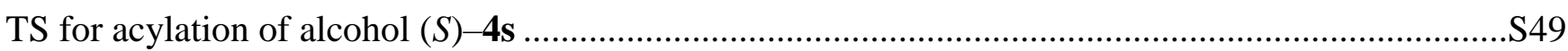

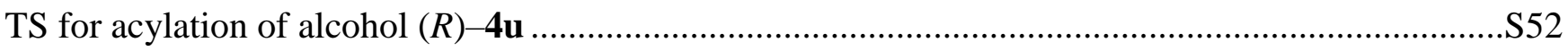

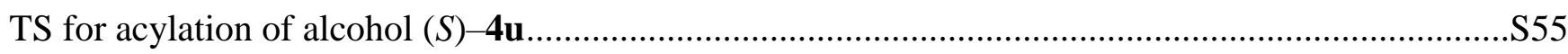

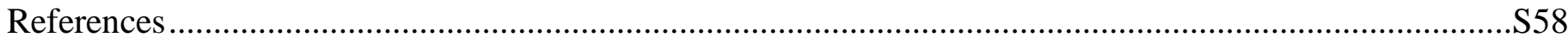

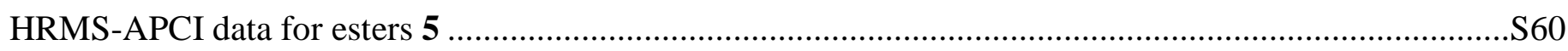



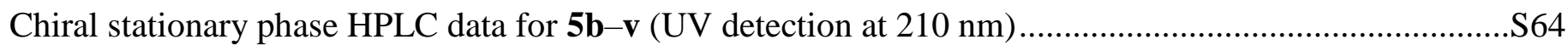






\section{Screening of Lewis base catalysts for KR of secondary alcohol 4a}

The screening of the influence of chiral LB catalysts on the selectivity of the KR of the secondary alcohol 4a was carried out by following General procedure A (Table S1). The KR was performed at room temperature with both acetic anhydride and isobutyric anhydride using a mixture of t-amyl alcohol and toluene $(1: 1 v / v)$ as the solvent. In most KR experiments, full consumption of the acylating reagent was observed by ${ }^{1} \mathrm{H}-\mathrm{NMR}$ assay within $4 \mathrm{~h}$. Therefore, the selectivity factor of the KR was determined by ${ }^{1} \mathrm{H}$ NMR and chiral GC assay after $4 \mathrm{~h}$. The NMR sample was prepared by diluting $50 \mathrm{uL}$ aliquot of the reaction mixture with $\mathrm{CDCl}_{3}$ without any additional workup. The conversion was determined by integrating the corresponding benzylic C-H signals for both alcohol $\mathbf{4 a}(\delta=4.9(1 \mathrm{H}, \mathrm{q}, J=6.4 \mathrm{~Hz}))$ and ester $5(\delta=$ $5.89(1 \mathrm{H}, \mathrm{q}, J=6.6 \mathrm{~Hz}))$. The chiral GC sample was prepared by diluting $20 \mathrm{uL}$ aliquot of the reaction mixture with $\mathrm{Et}_{2} \mathrm{O}(0.5 \mathrm{~mL})$. The solution was extracted with saturated solution of $\mathrm{NaHCO}_{3}(0.5 \mathrm{~mL})$. The organic layer was filtered through $\mathrm{MgSO}_{4}$ plug, diluted with $\mathrm{Et}_{2} \mathrm{O}$ to $1 \mathrm{~mL}$ and analyzed by chiral GC. The selectivity factor $(s)$ of the KR was calculated using the formula (1). ${ }^{1}$

$$
s=\frac{\ln (1-c(1+e e))}{\ln (1-c(1-e e))}
$$

Where:

c- conversion,

ee - enantiomeric excess of ester 5

Overall, the use of acetic anhydride effected KR with higher selectivity factor as compared to that using isobutyric anhydride. Use of Vedejs's catalysts $\mathbf{1 7}^{2}, \mathbf{1 8}^{3}$ and $\mathbf{1 9}^{4}$ in the KR provided the acylation of alcohol 4a with low selectivity $(s=1-2)$. Similarly, low selectivities $(s=1$ and 2$)$ of the acylation were achieved when Ishihara's $\mathbf{2 1}^{5}$, Mandai's $\mathbf{2 4}^{6}$ and Fu's $\mathbf{2 0}^{7}$ catalysts were employed in the KR of alcohol $4 \mathbf{a}$. Slightly higher selectivities of the acylation were achieved using Connon's catalyst $7^{8}(s=4)$, Vedejs's catalyst $\mathbf{6}^{3}(s=4)$ and HyperBTM catalyst $\mathbf{2 3}^{9}(s=9)$. The highest selectivities of the KR were achieved when BTM catalyst $\mathbf{2 2}^{10}(s=20)$, HyperBTM species $\mathbf{3}^{9}(s=20)$ and Fu's catalyst $\mathbf{8}^{7}(s=23)$ were used as chiral Lewis Base (LB) catalysts. However, the acylation of alcohol 4a using Fu's catalyst 8 was significantly slower (less than $2 \%$ conversion after $24 \mathrm{~h}$ ) as compared to the other catalysts (25\% conversion within 4 h). As a result, HyperBTM catalyst 3 was chosen for subsequent development of the DKR process. 


\section{Kinetic Resolution of secondary alcohol 4a}

Table S1. Kinetic resolution of secondary alcohol $\mathbf{4 a}$

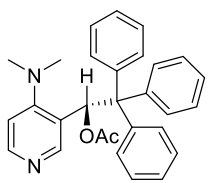

17

$s_{i-\operatorname{PrCO}}=2$

$s_{\mathrm{Ac}}=1$

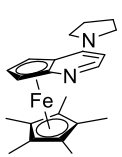

20

$s_{i-\operatorname{PrCO}}=1$
$s_{A C}=2$

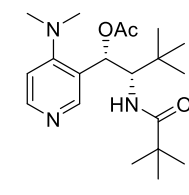

18

$s_{i-\operatorname{PrCO}}=1$

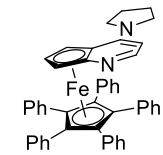

$s_{i-\mathrm{PrCO}}=5^{*}$

$s_{\mathrm{AC}}=23^{*}$

hen $2 \%$ conversion
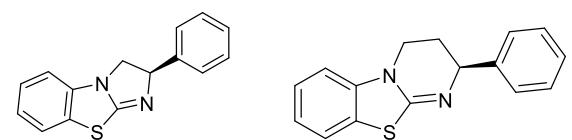

22

$s_{i-\operatorname{PrCO}}=6$
$s_{\mathrm{AC}}=20$

after $24 \mathrm{~h}$

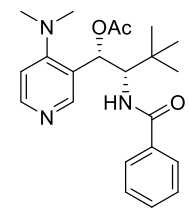

$s_{i-\mathrm{PrCO}}=3$

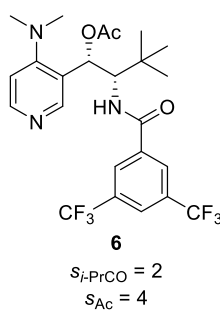

23

$s_{i-\operatorname{PrCO}}=4$
$s_{\mathrm{AC}}=9$
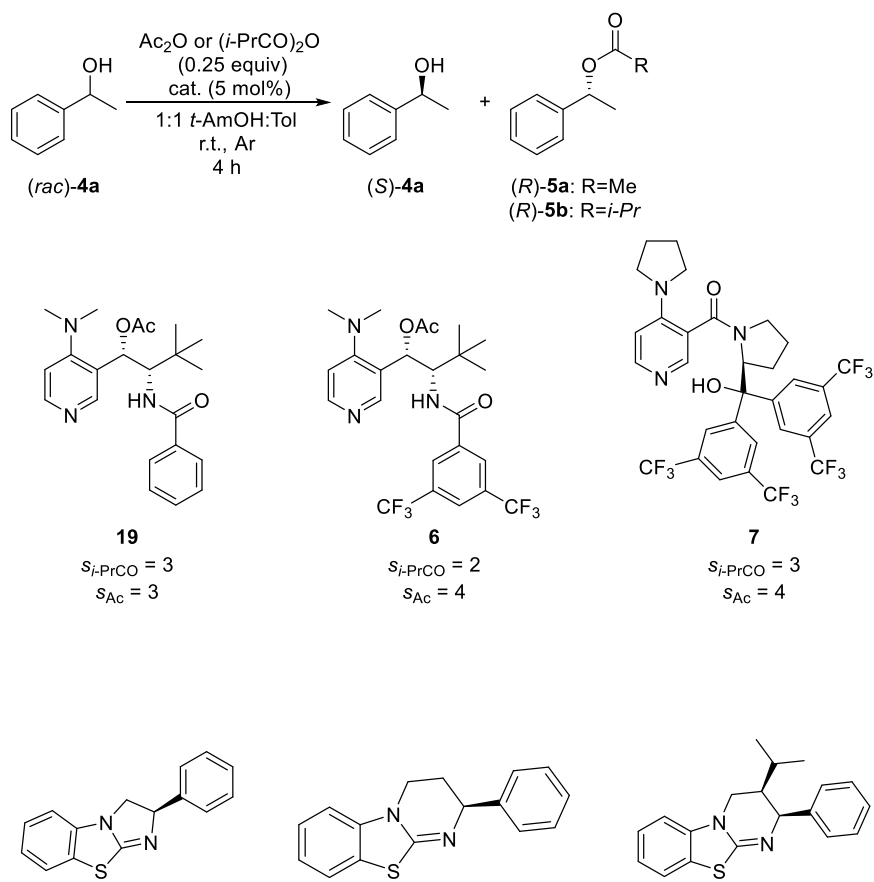

3

$s_{i-\operatorname{PrCO}}=10$
$s_{A C}=20$

7

$s_{i-\operatorname{PrCO}}=3$

$s_{\mathrm{AC}}=4$

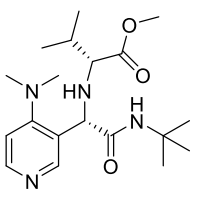

24

$s_{i-\mathrm{PrCO}}=2$

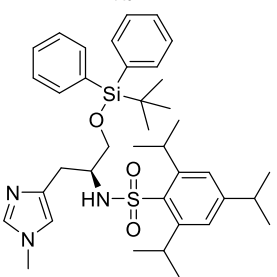

$s_{i-\operatorname{PrCO}}=1$

\begin{tabular}{ccc|cccc|cccc}
\hline \multirow{2}{*}{ entry } & \multirow{2}{*}{ catalyst } & \multirow{2}{*}{ time, h } & \multicolumn{4}{|c|}{ ester 5a } & \multicolumn{5}{c}{ ester 5b } \\
& & & $R: S$ & ee, \% & conv., \% & $s$ & $R: S$ & ee, \% & conv., \% & $s$ \\
\hline 1 & $\mathbf{1 7}$ & 4 & $58: 42$ & 16 & 25 & 1 & $67: 33$ & 34 & 25 & 2 \\
2 & $\mathbf{1 8}$ & 4 & $36: 64$ & 28 & 21 & 2 & $47: 53$ & 6 & 19 & 1 \\
3 & $\mathbf{1 9}$ & 4 & $72: 28$ & 44 & 25 & 3 & $69: 31$ & 38 & 25 & 3 \\
4 & $\mathbf{6}$ & 4 & $79: 21$ & 58 & 25 & 4 & $68: 32$ & 36 & 25 & 2 \\
5 & $\mathbf{7}$ & 4 & $23: 77$ & 54 & 25 & 4 & $27: 73$ & 46 & 25 & 3 \\
6 & $\mathbf{2 4}$ & 4 & $39: 61$ & 22 & 25 & 2 & $41: 59$ & 18 & 22 & 2 \\
7 & $\mathbf{2 0}$ & 4 & $64: 36$ & 28 & 23 & 2 & $53: 47$ & 6 & 13 & 1 \\
8 & $\mathbf{8}$ & 24 & $96: 4$ & 92 & 2 & 23 & $82: 18$ & 64 & 2 & 5 \\
9 & $\mathbf{2 2}$ & 4 & $94: 6$ & 88 & 25 & 20 & $84: 16$ & 68 & 25 & 6 \\
10 & $\mathbf{2 3}$ & 4 & $88: 12$ & 76 & 25 & 9 & $77: 23$ & 54 & 25 & 4 \\
11 & $\mathbf{3}$ & 4 & $94: 6$ & 88 & 25 & 20 & $88: 12$ & 76 & 25 & 10 \\
12 & $\mathbf{2 1}$ & 4 & $49: 51$ & 2 & 25 & 1 & $47: 53$ & 6 & 23 & 1 \\
\hline
\end{tabular}




\section{Screening of transition metal catalysts for the racemization of secondary alcohol 4a}

Racemization of enantiopure alcohol $(R)-\mathbf{4 a}$ was carried out by following General procedure B (Figure S1). The reaction was performed at room temperature using a 1:1 $(v / v)$ mixture of $t$-amyl alcohol and toluene as the solvent. For initial screening, the racemization was performed under an argon atmosphere. A variety of ruthenium ${ }^{11-16}$, iridium ${ }^{17,18}$ and $\operatorname{iron}^{19}$ complexes was tested as the racemization catalysts. However, only four catalysts - Bäckvall's catalyst $\mathbf{1},{ }^{20,21}$ Nolan's catalyst $\mathbf{9},{ }^{22}$ Park's catalyst $\mathbf{1 0}^{14}$ and catalyst $\mathbf{1 1}^{21}$ were able to fully racemize the alcohol $(R)-\mathbf{4 a}$. Out of these catalysts, only Bäckvall's catalyst $\mathbf{1}$ retained its activity when the racemization was performed without inert atmosphere. As a result, Bäckvall's catalyst 1 was chosen for subsequent development of the DKR process.

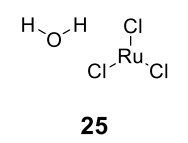

99:1 er after $24 \mathrm{~h}$

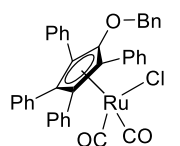

28

59:41 er afer $24 \mathrm{~h}$

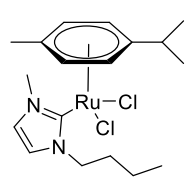

31

96:4 er after $24 \mathrm{~h}$

* Racemization of the alcohol was perormed withour ar

$85: 15$ er after $24 \mathrm{~h}$

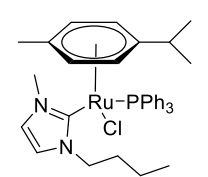

32

97:3 er after $24 \mathrm{~h}$


27

92:8 er after $24 \mathrm{~h}$

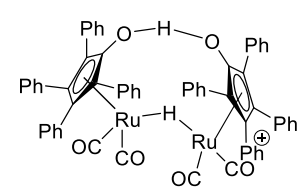

29

98:2 er after $24 \mathrm{~h}$

50:50 er after $30 \mathrm{~min}$

$75: 25$ er after $24 h^{*}$



95:5 er after $24 \mathrm{~h}$

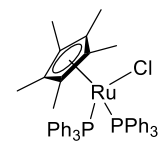

11

$55: 45$ er after $2 \mathrm{~h}$ $97: 3$ er after $2 h^{*}$

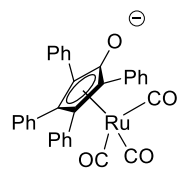

30

$85: 15$ er after $24 \mathrm{~h}$

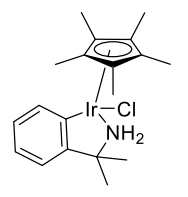

36

$89: 11$ er after $24 \mathrm{~h}$

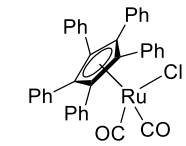

1

50:50 er after $30 \mathrm{~min}$ 50:50 er after $30 \mathrm{~min}^{*}$



34

98:2 er after $24 \mathrm{~h}$

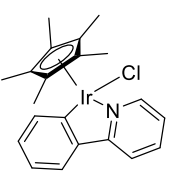

37

98:2 er after $24 \mathrm{~h}$

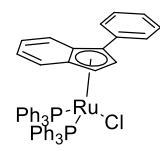

9

50:50 er after $30 \mathrm{~min}$ 94:6 er after $30 \mathrm{~min}^{*}$

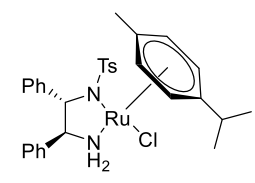

35 $75: 25$ er after $24 \mathrm{~h}$

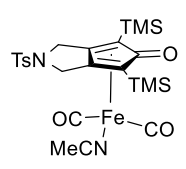

38

99:1 er after $24 \mathrm{~h}$

Figure S1. Racemization of secondary alcohol $(R)-\mathbf{4 a}$ 


\section{Pre-activation of Bäckvall's catalyst 1}

The influence of pre-activation of Bäckvall's catalyst $\mathbf{1}$ on the rate of racemization of alcohol $\mathbf{4 a}$ was determined by following General procedure B. The experiment was carried out by varying stirring time of the suspension comprising Bäckvall's catalyst $\mathbf{1}$ and $t$-BuOK before the addition of enantiopure alcohol $(R)-4 a$. The enantiomeric ratio of alcohol $\mathbf{4 a}$ was determined by chiral GC analysis 30 minutes after alcohol 4a was added to the suspension. The graph below clearly illustrates that at least 15 minutes of the preactivation of the ruthenium catalyst is required to achieve a maximum rate of alcohol $\mathbf{4 a}$ racemization.

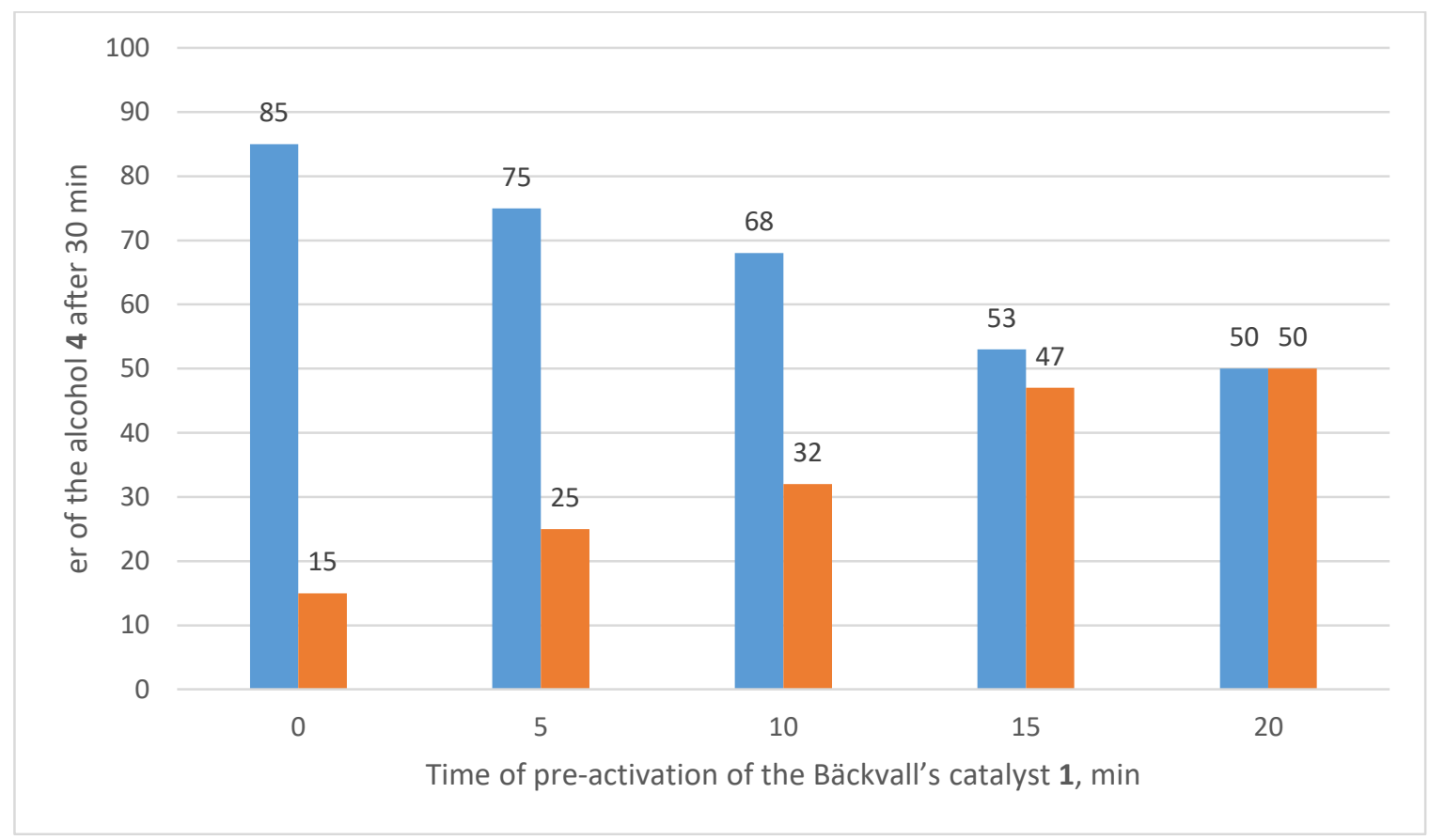

Figure S2. Influence of pre-activation of Bäckvall's catalyst 1 on the rate of racemization of alcohol $\mathbf{4 a}$ 


\section{Screening of the compatibility of racemization conditions and acylating reagents}

The influence of an acylating reagent on the racemization of alcohol $(R)-\mathbf{4 a}$ was assessed by adding an associated anion of the acylating reagent to the racemization reaction under conditions of General procedure C (Table S2). The racemization of $(R)-\mathbf{4 a}$ was not observed when pivalate (entry 3), pentafluorophenolate (entry 5) or $1 H$-benzo $[d][1,2,3]$ triazol-1-olate (entry 6) anions were added to the racemization mixture. In contrast, alcohol $(R)-\mathbf{4 a}$ was fully racemized in the presence of benzoate (entry 1 ), 4-nitrobenzoate (entry 2), 4-nitrophenolate (entry 4) and 2,5-dioxopyrrolidin-1-olate (entry 7) anions.

Table S2. Racemization of alcohol (R)-4a in the presence of additives

entry

${ }^{a}$ Formation of acylated alcohol 5a was not observed when 2,5-dioxopyrrolidin-1-yl acetate was employed as acylating reagent under KR conditions (General procedure A) 


\section{Optimization of the DKR conditions}

Table S3. Optimization of the DKR conditions for alcohol ( rac)-4a

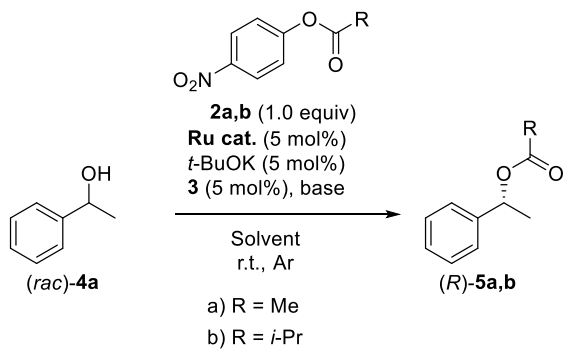

\begin{tabular}{|c|c|c|c|c|c|c|c|}
\hline entry & $\mathbf{R}$ & Base & Solvent & Ru cat. & Conv. after 24 h, \% & 4 (er) & 5 (er) \\
\hline 1 & $\mathrm{Me}$ & - & 1:1 $t$-AmOH:toluene & 1 & 22 & $53: 47$ & $84: 16$ \\
\hline 2 & $\mathrm{Me}$ & - & Toluene & 1 & 35 & $51: 49$ & $86: 14$ \\
\hline 3 & $\mathrm{Me}$ & - & $\mathrm{PhCF}_{3}$ & 1 & 47 & $56: 44$ & $87: 13$ \\
\hline 4 & $\mathrm{Me}$ & - & MTBE & 1 & 45 & $61: 39$ & $86: 14$ \\
\hline 5 & $\mathrm{Me}$ & - & DCM & 1 & 54 & $55: 45$ & $85: 15$ \\
\hline 6 & $\mathrm{Me}$ & - & EtOAc & 1 & 39 & $62: 38$ & $80: 20$ \\
\hline 7 & $\mathrm{Me}$ & - & $\mathrm{MeCN}$ & 1 & 41 & $66: 34$ & $73: 27$ \\
\hline 8 & $\mathrm{Me}$ & - & Benzene & 1 & 33 & $52: 48$ & $86: 14$ \\
\hline 9 & $\mathrm{Me}$ & - & neat & 1 & 59 & $60: 40$ & $77: 23$ \\
\hline 10 & $\mathrm{Me}$ & $\mathrm{K}_{2} \mathrm{CO}_{3}$ & 1:1 $t$-AmOH:toluene & 1 & 41 & $51: 49$ & $89: 11$ \\
\hline 11 & $\mathrm{Me}$ & $\mathrm{K}_{3} \mathrm{PO}_{4}$ & 1:1 $t$-AmOH:toluene & 1 & 49 & $58: 42$ & $82: 18$ \\
\hline 12 & $\mathrm{Me}$ & $\mathrm{Li}_{2} \mathrm{CO}_{3}$ & 1:1 t-AmOH:toluene & 1 & 39 & $54: 46$ & $87: 13$ \\
\hline 13 & $\mathrm{Me}$ & Proton Sponge & 1:1 $t$-AmOH:toluene & 1 & 31 & $52: 48$ & $88: 12$ \\
\hline 14 & $\mathrm{Me}$ & TMG & 1:1 $t$-AmOH:toluene & 1 & 13 & $52: 48$ & $78: 22$ \\
\hline 15 & $\mathrm{Me}$ & $\mathrm{NEt}_{3}$ & 1:1 t-AmOH:toluene & 1 & 29 & $51: 49$ & $87: 13$ \\
\hline 16 & $\mathrm{Me}$ & DIPEA & 1:1 $t$-AmOH:toluene & 1 & 26 & $52: 48$ & $87: 13$ \\
\hline 17 & $\mathrm{Me}$ & DABCO & 1:1 $t$-AmOH:toluene & 1 & 29 & $56: 44$ & $83: 17$ \\
\hline 18 & $\mathrm{Me}$ & $\mathrm{K}_{2} \mathrm{CO}_{3}$ & $\mathrm{PhCF}_{3}$ & 1 & 56 & $51: 49$ & $89: 11$ \\
\hline 19 & $\mathrm{Me}$ & $\mathrm{NEt}_{3}$ & $\mathrm{PhCF}_{3}$ & 1 & 36 & $53: 47$ & $87: 13$ \\
\hline 20 & $\mathrm{Me}$ & Proton Sponge & $\mathrm{PhCF}_{3}$ & 1 & 38 & $51: 49$ & $88: 12$ \\
\hline 21 & $\mathrm{Me}$ & $\mathrm{K}_{2} \mathrm{CO}_{3}$ & 1:1 $\mathrm{PhCF}_{3}:$ Toluene & 1 & 35 & $52: 48$ & $87: 13$ \\
\hline 22 & $\mathrm{Me}$ & $\mathrm{K}_{2} \mathrm{CO}_{3}$ & 1:1 $\mathrm{PhNO}_{2}:$ Toluene & 1 & 55 & $52: 48$ & $87: 13$ \\
\hline 23 & $\mathrm{Me}$ & $\mathrm{K}_{2} \mathrm{CO}_{3}$ & 1:1 Pyridine:Toluene & 1 & 15 & $55: 45$ & $77: 13$ \\
\hline 24 & $\mathrm{Me}$ & $\mathrm{K}_{2} \mathrm{CO}_{3}$ & 1,2-difluorobenzene & 1 & 47 & $53: 47$ & $88: 12$ \\
\hline 25 & $\mathrm{Me}$ & $\mathrm{K}_{2} \mathrm{CO}_{3}$ & 1,2-dichlorobenzene & 1 & 52 & $58: 42$ & $87: 13$ \\
\hline 26 & $\mathrm{Me}$ & $\mathrm{K}_{2} \mathrm{CO}_{3}$ & 1:9 DMA:Toluene & 1 & 31 & $51: 49$ & $84: 16$ \\
\hline 27 & $i$-Pr & $\mathrm{K}_{2} \mathrm{CO}_{3}$ & $\mathrm{PhCF}_{3}$ & 1 & 50 & $51: 49$ & $96: 4$ \\
\hline 28 & $i$-Pr & $\mathrm{K}_{2} \mathrm{CO}_{3}$ & $\mathrm{PhCF}_{3}$ & 11 & 42 & $66: 34$ & $84: 16$ \\
\hline 29 & $i$-Pr & $\mathrm{K}_{2} \mathrm{CO}_{3}$ & $\mathrm{PhCF}_{3}$ & 10 & 46 & $53: 47$ & $95: 5$ \\
\hline $30^{b}$ & $i$-Pr & $\mathrm{K}_{2} \mathrm{CO}_{3}$ & $\mathrm{PhCF}_{3}$ & 1 & 55 & $54: 46$ & $96: 4$ \\
\hline $31^{b}$ & $i$-Pr & $\mathrm{K}_{2} \mathrm{CO}_{3}$ & $\mathrm{PhCF}_{3}$ & 10 & 47 & $67: 33$ & $82: 18$ \\
\hline 32 & $t-\mathrm{Bu}$ & $\mathrm{K}_{2} \mathrm{CO}_{3}$ & $\mathrm{PhCF}_{3}$ & 1 & $<1$ & $50: 50$ & $\mathrm{n} / \mathrm{a}$ \\
\hline
\end{tabular}

${ }^{a}$ Determined by ${ }^{l} H$-NMR assay after $24 \mathrm{~h}$; reactions were performed on 0.4 mmol scale ${ }^{b}$ The DKR was performed under ambient atmosphere. 


\section{General procedures}

General procedure A. Racemic alcohol 4a (1.0 equiv) and an acylating reagent ( 0.25 equiv) were added to a solution of Lewis base catalyst ( 0.05 equiv) in $t-\mathrm{AmOH} /$ toluene mixture (1/1 (v/v), $0.8 \mathrm{M}$ solution relative to the alcohol $\mathbf{4 a}$ ). The solution was stirred at room temperature under an Ar atmosphere. The reaction progress was monitored by ${ }^{1} \mathrm{H}-\mathrm{NMR}$ analysis and chiral GC analysis.

General procedure B. Racemization catalyst ( 0.02 equiv) and $t$ - $\mathrm{BuOK}$ (stored in a glovebox, 0.02 equiv) were suspended in $t-\mathrm{AmOH} /$ toluene mixture (1/1 (v/v), $0.8 \mathrm{M}$ solution relative to the alcohol 4a). The light-yellow suspension was stirred under an Ar atmosphere for 15 minutes, then the enantiopure alcohol $4 \mathbf{a}$ (1.0 equiv) was added to the dark brown suspension. The suspension was stirred at room temperature. Progress of the racemization was monitored by chiral GC analysis.

General procedure C. Bäckvall's catalyst (0.05 equiv) and $t$-BuOK (stored in a glovebox, 1.05 equiv) were suspended in 1:1 $t$-AmOH:toluene mixture, $0.8 \mathrm{M}$ solution relative to the alcohol 4a). The light-yellow suspension was stirred under an Ar atmosphere for 15 minutes, then the additive (1.0 equiv) and enantiopure alcohol 4a (1.0 equiv) was added to the dark brown suspension. The suspension was stirred at room temperature. Progress of the racemization was monitored by chiral GC analysis. 


\section{DFT calculations}

All calculations were performed using Gaussian 09 software package. ${ }^{23}$ Transition state (TS) geometries were located using the Berny algorithm. Intrinsic reaction coordinates (IRC) were calculated for the transition states to confirm that the saddle point connected the correct reactants and products on the potential energy surface. Optimizations of stationary points were performed without any restrictions using density-functional theory method B3LYP/6-31g(d). For stationary points that had multiple conformations, the lowest-energy conformation was identified by comparing energies of the structures optimized from different starting points. Stationary points were verified to be real minima (zero imaginary frequency) or TS (one imaginary frequency) by performing frequency calculations at the same level of theory. The thermochemical analysis was done at 298.15 K. Single-point energy calculations were performed on the stationary points using B3LYP/6-311++g(2df,2p) method. Thermal correction to Gibbs free energy from lower-level frequency calculations combined with single-point energies was used to describe the energetics of the acylation reaction. For all calculations, a superfine integral grid was applied (integral=grid=superfine) ${ }^{24}$

To simplify the computational model, two approximations were made: (a) the formation of acetic acid ester was calculated instead of isobutyric acid ester; (b) acetate was used as the counter-ion instead of 4-nitrophenolate or carbonate. The validity of the approximation for the counter-ion choice was tested by performing DFT calculations for the acylation of alcohol 4a with both acetate and 4-nitrophenolate as counter-ions. Calculated Gibbs free energy difference $(\Delta \Delta \mathrm{G})$ for the diastereomeric TS with acetate counterion was $2.5 \mathrm{kcal} / \mathrm{mol}$, and free energy difference $(\Delta \Delta \mathrm{G})$ for the diastereomeric TS with 4-nitrophenolate counter-ion was $2.3 \mathrm{kcal} / \mathrm{mol}$. Apparently, counter-ion has small influence on the overall selectivity of the acylation. Consequently, all subsequent calculations were performed using acetate as the counter-ion. 


\section{TS for acylation of alcohol $(R)-4 a$}

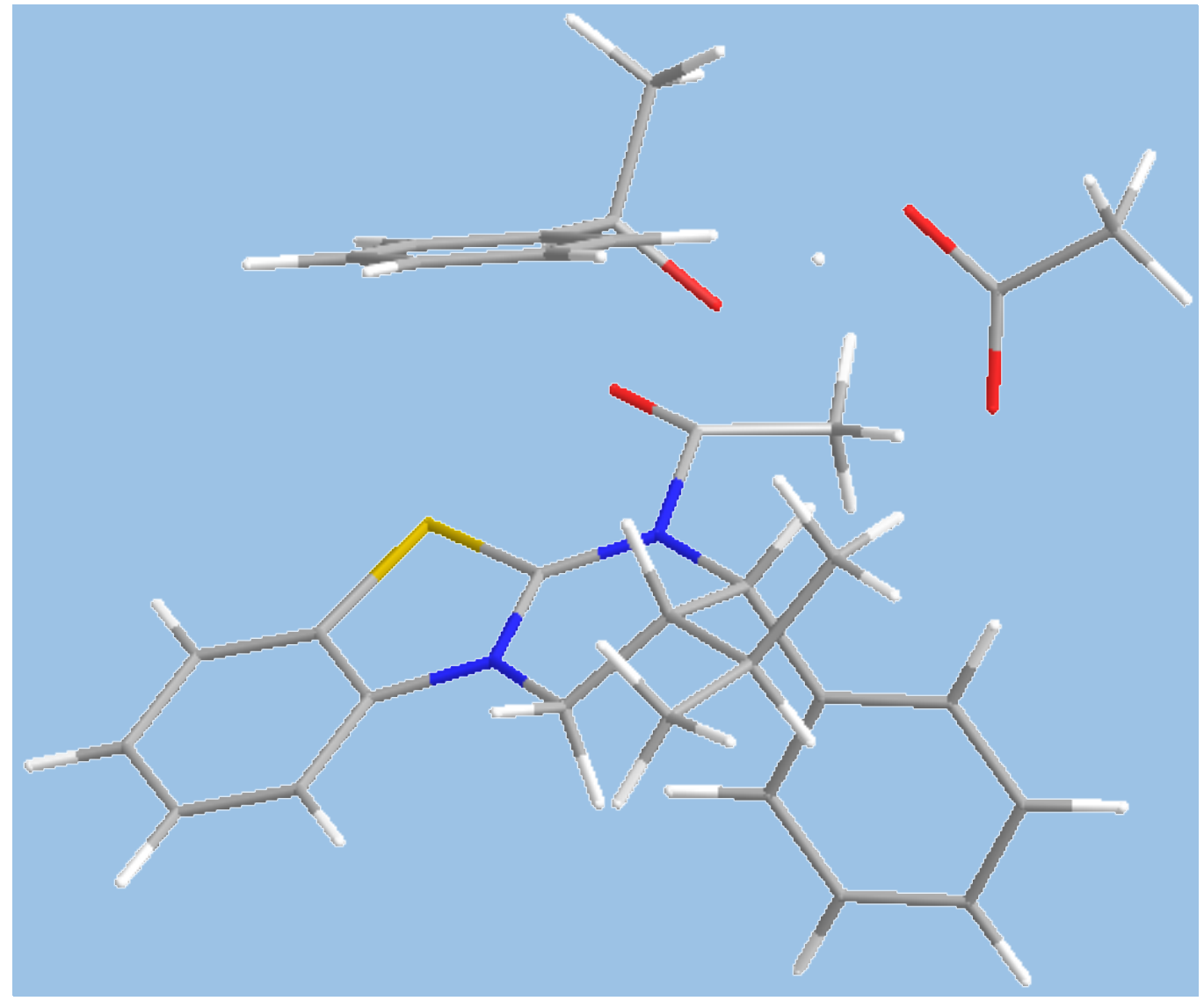

Zero-point correction=

Thermal correction to Energy=

Thermal correction to Enthalpy=

Thermal correction to Gibbs Free Energy=

Sum of electronic and zero-point Energies=

Sum of electronic and thermal Energies=

Sum of electronic and thermal Enthalpies=

Sum of electronic and thermal Free Energies=

$E(R B 3 L Y P)=-2012.16183472$

Number of imaginary frequencies: 1

Charge $=0$ Multiplicity $=1$

$\begin{array}{lccc}\mathrm{C} & -0.52016 & 0.09247 & -2.20603 \\ \mathrm{C} & -1.74001 & 0.86606 & -2.64225 \\ \mathrm{H} & -2.47499 & 1.02667 & -1.85478 \\ \mathrm{O} & 0.22054 & -0.46814 & -3.01126 \\ \mathrm{~N} & 0.14795 & 0.57326 & -0.9634 \\ \mathrm{C} & 1.44552 & 0.339 & -0.81375 \\ \mathrm{~N} & 2.1171 & 0.64466 & 0.32257 \\ \mathrm{C} & -0.04113 & 0.70949 & 1.49789\end{array}$

0.612442 (Hartree/Particle)

0.649215

0.650159

0.541210

$-2010.981033$

$-2010.944260$

$-2010.943316$

$-2011.052265$ 


$$
\begin{aligned}
& \begin{array}{lll}
-0.64397 & 1.18546 & 0.14505
\end{array} \\
& \begin{array}{llll}
-0.7559 & 2.69901 & -0.02265
\end{array} \\
& \begin{array}{lll}
-1.99759 & 3.31175 & 0.20854
\end{array} \\
& \begin{array}{lll}
-2.13434 & 4.69721 & 0.10755
\end{array} \\
& \begin{array}{lll}
-1.04127 & 5.49279 & -0.23811
\end{array} \\
& \begin{array}{lll}
0.19167 & 4.89155 & -0.49341
\end{array} \\
& \begin{array}{llll}
0.33204 & 3.50683 & -0.38749
\end{array} \\
& \begin{array}{llll}
1.29619 & 3.06196 & -0.61781
\end{array} \\
& \begin{array}{lll}
1.04626 & 5.49761 & -0.78265
\end{array} \\
& \begin{array}{lll}
-1.15162 & 6.5706 & -0.3222
\end{array} \\
& \begin{array}{lll}
-3.1049 & 5.15241 & 0.28609
\end{array} \\
& \begin{array}{lll}
-2.86222 & 2.69437 & 0.43233
\end{array} \\
& \begin{array}{lll}
-1.63783 & 0.74544 & 0.05824
\end{array} \\
& \begin{array}{lll}
-0.10815 & -0.38684 & 1.47985
\end{array} \\
& \begin{array}{lll}
-1.55797 & -1.34686 & -1.21184
\end{array} \\
& \begin{array}{llll}
-0.98006 & -2.62107 & -1.37918
\end{array} \\
& \begin{array}{lll}
-0.51038 & -3.19363 & -0.04304
\end{array} \\
& \begin{array}{lll}
0.75275 & -3.78236 & 0.08762
\end{array} \\
& \begin{array}{lll}
1.17435 & -4.321 & 1.30674
\end{array} \\
& \begin{array}{lll}
0.33308 & -4.27531 & 2.41872
\end{array} \\
& \begin{array}{lll}
-0.93128 & -3.68944 & 2.30111
\end{array} \\
& \begin{array}{lll}
-1.34757 & -3.15229 & 1.08254
\end{array} \\
& \begin{array}{lll}
-2.32904 & -2.69509 & 0.99128
\end{array} \\
& \begin{array}{lll}
-1.5963 & -3.65627 & 3.16121
\end{array} \\
& \begin{array}{lll}
0.65649 & -4.69411 & 3.36847
\end{array} \\
& \begin{array}{lll}
-0.09543 & -2.49889 & -2.02488
\end{array} \\
& \begin{array}{lll}
-2.80255 & -1.34686 & -1.05312
\end{array} \\
& \begin{array}{lll}
-3.95867 & -1.43168 & -0.85926
\end{array} \\
& \begin{array}{lll}
-4.49134 & -0.37192 & -0.33157
\end{array} \\
& \begin{array}{lll}
-5.97919 & -0.50631 & -0.04473
\end{array} \\
& \begin{array}{lll}
-3.88431 & 0.67837 & -0.06782
\end{array} \\
& \begin{array}{lll}
2.49741 & -0.33983 & -2.05536
\end{array} \\
& \begin{array}{lll}
3.50296 & 0.432 & 0.25469
\end{array} \\
& \begin{array}{lll}
3.89 & -0.09848 & -0.98014
\end{array} \\
& \begin{array}{lll}
5.22761 & -0.38964 & -1.23921
\end{array} \\
& \begin{array}{lll}
5.52846 & -0.80685 & -2.19574
\end{array} \\
& \begin{array}{lll}
4.44096 & 0.69758 & 1.25374
\end{array} \\
& \begin{array}{lll}
4.14679 & 1.12315 & 2.20704
\end{array} \\
& \begin{array}{lll}
5.77944 & 0.40813 & 0.98727
\end{array} \\
& \begin{array}{lll}
6.52592 & 0.60811 & 1.74996
\end{array} \\
& \begin{array}{lll}
6.17122 & -0.13081 & -0.24423
\end{array} \\
& \begin{array}{lll}
7.21872 & -0.3482 & -0.42958
\end{array} \\
& \begin{array}{lll}
-1.93598 & -3.58871 & -2.10138
\end{array}
\end{aligned}
$$


H

$\begin{array}{ccc}-6.37944 & 0.42928 & 0.35019 \\ -6.14495 & -1.31285 & 0.6782 \\ -6.51134 & -0.78306 & -0.96094 \\ 2.16013 & -4.77344 & 1.38667 \\ 1.41359 & -3.82071 & -0.77659 \\ -2.19841 & 0.29707 & -3.45279 \\ -1.40915 & 1.8345 & -3.03852 \\ -1.4568 & -4.56151 & -2.26282 \\ -2.21375 & -3.17012 & -3.07483 \\ -2.85147 & -3.745 & -1.52197 \\ 1.44026 & 1.08092 & 1.55217 \\ 1.58149 & 2.16174 & 1.6752 \\ 1.9363 & 0.57046 & 2.37915 \\ -0.81128 & 1.21456 & 2.7489 \\ -0.94964 & 2.299 & 2.64462 \\ -0.03057 & 0.96685 & 4.05387 \\ -0.64534 & 1.25706 & 4.91269 \\ -0.89803 & 1.54584 & 4.11569 \\ -0.21852 & -0.09569 & 4.17338 \\ -2.1954 & 0.55337 & 2.85846 \\ -2.81919 & 0.70762 & 1.97469 \\ -2.08849 & -0.52892 & 3.00602\end{array}$




\section{TS for acylation of alcohol $(S)-4 a$}

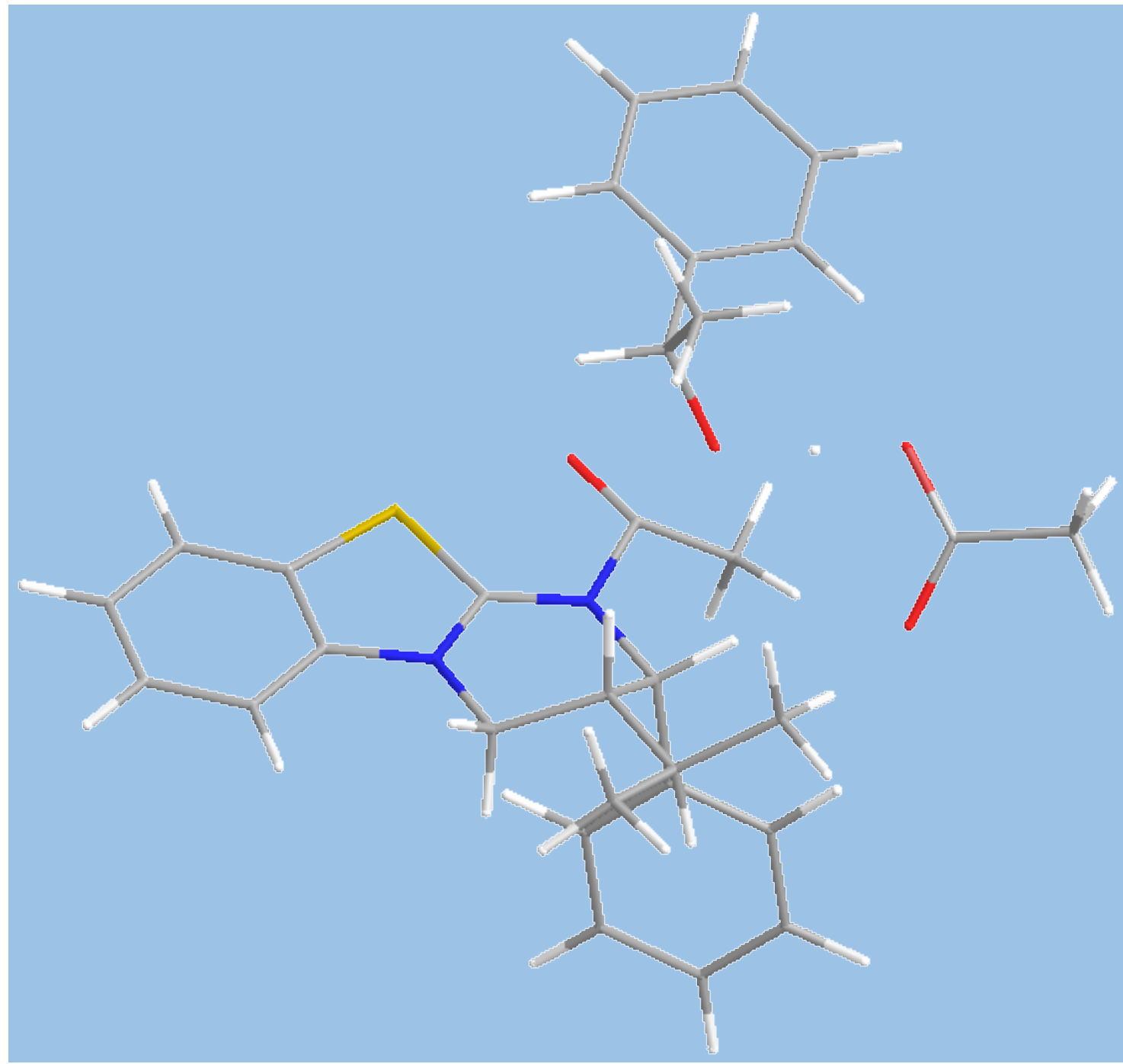

Zero-point correction=

Thermal correction to Energy=

Thermal correction to Enthalpy=

Thermal correction to Gibbs Free Energy=

Sum of electronic and zero-point Energies=

Sum of electronic and thermal Energies=

Sum of electronic and thermal Enthalpies=

Sum of electronic and thermal Free Energies=

$E(R B 3 L Y P)=-2012.15725555$

Number of imaginary frequencies: 1
0.612311 (Hartree/Particle)

0.649115

0.650059

0.540668

$-2010.976537$

$-2010.939733$

$-2010.938789$

$-2011.048180$

Charge $=0$ Multiplicity $=1$

$$
-0.8313
$$$$
-0.35508-1.22856
$$

C

$-1.42406$

0.77142

$-2.0407$

$\mathrm{H}$

$$
-1.47346
$$$$
1.72474
$$

$-1.51657$

O

$$
-0.96402-1.53289-1.55423
$$


$0.46621-0.04621-0.539$

$1.30653-1.04619-0.31218$

$\begin{array}{lll}2.45961 & -0.88941 & 0.38443\end{array}$

$1.53344 \quad 1.18724 \quad 1.31761$

$0.76534 \quad 1.32327 \quad-0.02933$

$\begin{array}{lll}1.46489 & 2.17231 & -1.08927\end{array}$

$1.07065 \quad 3.51028 \quad-1.24826$

$1.71197 \quad 4.33308-2.17565$

$2.74628 \quad 3.83395 \quad-2.96828$

$\begin{array}{lll}3.12999 & 2.49919 & -2.83487\end{array}$

$2.49375 \quad 1.67597 \quad-1.90428$

$2.79388 \quad 0.63377 \quad-1.84049$

$3.92056 \quad 2.09225-3.45986$

$3.23975 \quad 4.47464 \quad-3.69416$

$1.38908 \quad 5.36476 \quad-2.28724$

$\begin{array}{llll}0.23608 & 3.89159 & -0.6681\end{array}$

$\begin{array}{lll}-0.20711 & 1.76576 & 0.18999\end{array}$

$\begin{array}{lll}0.86633 & 0.61545 & 1.9783\end{array}$

$\begin{array}{lll}-1.91411 & -0.00851 & 0.41189\end{array}$

$-2.38082-1.20475 \quad 1.00065$

$\begin{array}{lll}-3.59279 & -1.82224 & 0.2999\end{array}$

$\begin{array}{lll}-4.66437 & -1.04093 & -0.15375\end{array}$

$\begin{array}{lll}-5.77841 & -1.63935 & -0.74544\end{array}$

$\begin{array}{lll}-5.84435 & -3.02617 & -0.89226\end{array}$

$\begin{array}{lll}-4.7826 & -3.813-0.44356\end{array}$

$\begin{array}{lll}-3.66855 & -3.21162 & 0.14311\end{array}$

$\begin{array}{lll}-2.83774 & -3.83047 & 0.47714\end{array}$

$\begin{array}{lll}-4.81644 & -4.89388 & -0.55914\end{array}$

$\begin{array}{lll}-6.71262 & -3.48868 & -1.35518\end{array}$

$\begin{array}{lll}-1.56895 & -1.94582 & 0.93533\end{array}$

$\begin{array}{lll}-2.67597 & 0.99061 & 0.57657\end{array}$

$\begin{array}{lll}-3.4343 & 1.85606 & 0.7794\end{array}$

$\begin{array}{lll}-2.9636 & 3.05117 & 0.58661\end{array}$

$\begin{array}{lll}-3.95937 & 4.16175 & 0.88021\end{array}$

$\begin{array}{lll}-1.81397 & 3.3085 & 0.19872\end{array}$

$1.09702-2.69424-0.90655$

$\begin{array}{lll}3.27436 & -2.03231 & 0.42782\end{array}$

$2.68671-3.11617-0.23221$

$3.3363-4.34743-0.28885$

$2.87886-5.19168-0.79638$

$\begin{array}{lll}4.52712 & -2.14544 & 1.03294\end{array}$

$4.99355-1.30232 \quad 1.53115$

$\begin{array}{lll}5.1746 & -3.37981 & 0.96914\end{array}$

\section{S14}


$\mathrm{H}$

C

$\mathrm{H}$

C

$\mathrm{H}$

$\mathrm{H}$

$\mathrm{H}$

$\mathrm{H}$

$\mathrm{H}$

$\mathrm{H}$

$\mathrm{H}$

$\mathrm{H}$

$\mathrm{H}$

$\mathrm{H}$

C

$\mathrm{H}$

H

C

$\mathrm{H}$

C

$\mathrm{H}$

H

H

C

H

$\mathrm{H}$

H

\begin{tabular}{|c|c|c|}
\hline 6.15073 & -3.48979 & 1.431 \\
\hline .58728 & -4.47093 & 0.3174 \\
\hline 10934 & -5.42221 & 0.2801 \\
\hline .67534 & -0.95741 & 2.49194 \\
\hline 51685 & 5.13749 & 0.67 \\
\hline 56 & 4.11213 & 6 \\
\hline 3 & 4.02398 & 0 \\
\hline 9999 & -1.01712 & 06 \\
\hline 2998 & 0.03816 & -0 \\
\hline 43167 & 0.45711 & $\begin{array}{ll}-2 & 31\end{array}$ \\
\hline 82828 & 0.88587 & -295 \\
\hline 7786 & -0.57229 & 8 \\
\hline ) & -1.88297 & \\
\hline .4750 & -0.21844 & 50624 \\
\hline .79354 & 0.35052 & 1 \\
\hline 年 & 0.904 & \\
\hline 2. & 0.054 & \\
\hline 8468 & 2.5403 & \\
\hline 307 & 3.20767 & \\
\hline 363 & 2.3897 & 7 \\
\hline & 3.3510 & 2 \\
\hline 85662 & 2.07769 & \\
\hline .51755 & 1.66635 & \\
\hline .56796 & 3.19958 & 255 \\
\hline 0.19211 & 3.36222 & 1.78847 \\
\hline ar & 4.1712 & 4 \\
\hline 208 & .577 & \\
\hline
\end{tabular}




\section{TS for acylation of alcohol $(\boldsymbol{R})-4$ a with 4-nitrophenolate counter-ion}

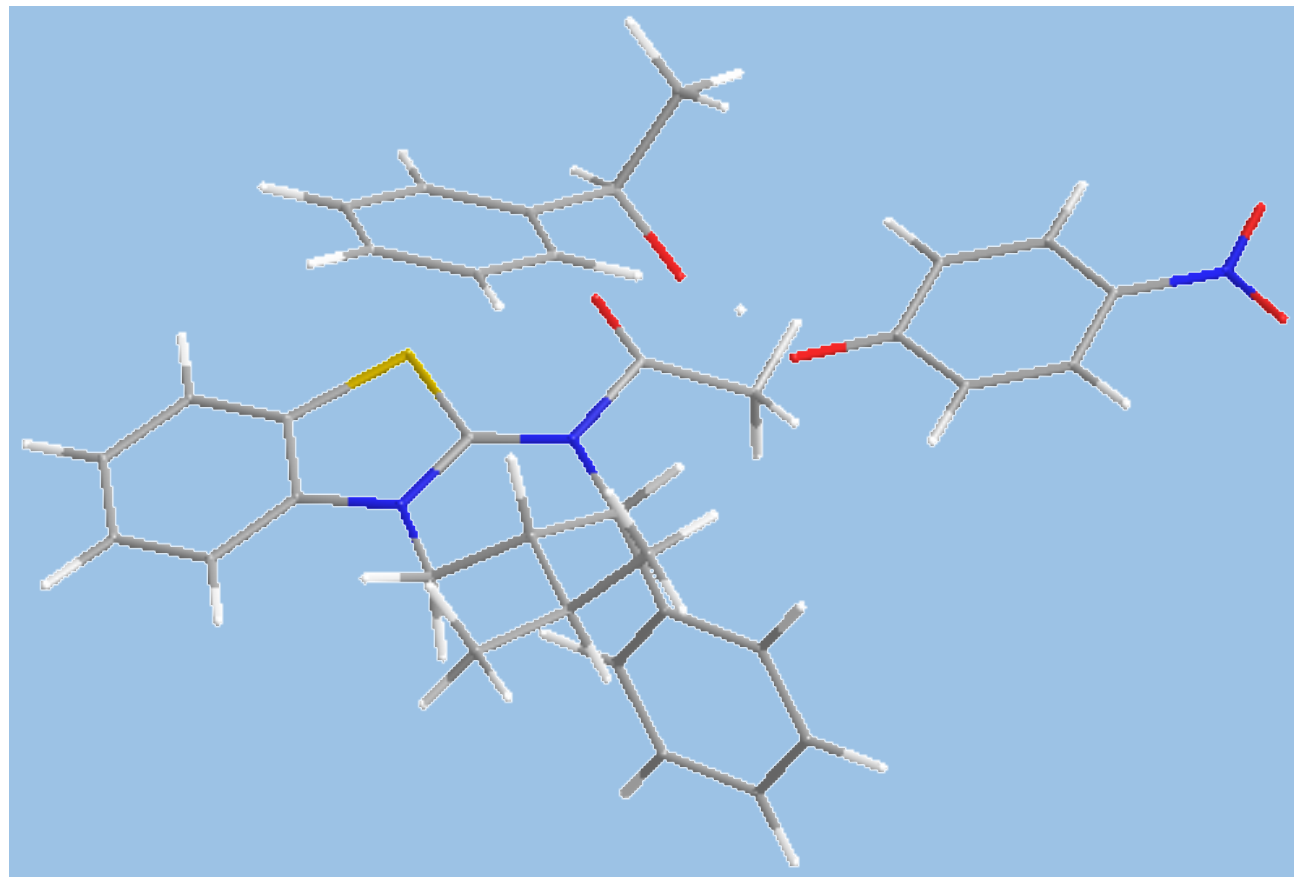

Zero-point correction=

Thermal correction to Energy=

Thermal correction to Enthalpy=

Thermal correction to Gibbs Free Energy=

Sum of electronic and zero-point Energies=

Sum of electronic and thermal Energies=

Sum of electronic and thermal Enthalpies=

Sum of electronic and thermal Free Energies=

\begin{abstract}
0.657629 (Hartree/Particle)
\end{abstract}
0.698212

0.699156

0.580175

$-2293.820760$

$-2293.780176$

$-2293.779232$

$-2293.898213$

$E(R B 3 L Y P)=-2295.13946807$

Number of imaginary frequencies: 1

Charge $=0$ Multiplicity $=1$

$\begin{array}{lrrr}\text { C } & 0.22396800 & 0.00666100 & -2.03610900 \\ \mathrm{C} & -1.01682800 & 0.81347700 & -2.32700900 \\ \mathrm{H} & -1.63345000 & 1.01222800 & -1.45312300 \\ \mathrm{O} & 0.79856100 & -0.64651500 & -2.89796900 \\ \mathrm{~N} & 1.07283000 & 0.48230000 & -0.91758400 \\ \mathrm{C} & 2.37885100 & 0.21878300 & -0.95792100 \\ \mathrm{~N} & 3.23077900 & 0.60358900 & 0.01950400 \\ \mathrm{C} & 1.31038700 & 0.84571600 & 1.52738900 \\ \mathrm{C} & 0.47256200 & 1.17998900 & 0.25907900 \\ \mathrm{C} & 0.27775100 & 2.66759400 & -0.01381800 \\ \mathrm{C} & -0.91009100 & 3.28028300 & 0.41178900 \\ \mathrm{C} & -1.12166600 & 4.64463200 & 0.21258100\end{array}$




\begin{tabular}{|c|c|c|}
\hline-0.15197600 & 5.41893300 & -0.42650400 \\
\hline 1.02662900 & 4.81777400 & -0.86951000 \\
\hline 1.23916700 & 3.45297700 & -0.66568200 \\
\hline 2.15252000 & 3.00220800 & -1.0445720 \\
\hline 1.78109600 & 5.40796800 & -1.3825310 \\
\hline .31872200 & 6.48001600 & -0.58905800 \\
\hline .05100200 & 5.09797500 & 0.54 \\
\hline 1.67965400 & 2.67944700 & 0.88 \\
\hline 0.50167600 & 0.70998300 & 0.3 \\
\hline 1.25804200 & -0.24483200 & 1.6 \\
\hline .86153600 & -1.37221700 & -0.8 \\
\hline .33918400 & -2.67802600 & -0.89997000 \\
\hline 0.50043200 & -3.00018700 & 0.3 \\
\hline 1.78490700 & -3.54280800 & 0.20750200 \\
\hline 2.54965600 & -3.86814200 & 1.3 \\
\hline 2.03577800 & -3.65437900 & 2.61 \\
\hline 0.75434900 & -3.11329000 & 2.7 \\
\hline 0.00515900 & -2.78642700 & 1.62 \\
\hline 0.99452400 & -2.35304900 & 1.7 \\
\hline 0.34157400 & -2.95283800 & 3.74821500 \\
\hline 2.62357400 & -3.91194900 & 3.48837300 \\
\hline 0.32607400 & -2.74192400 & -1.77626900 \\
\hline 1.71591400 & -1.04307600 & -0.06821000 \\
\hline 2.44099800 & -0.54541600 & 0.73205200 \\
\hline 3.19752300 & -0.60466600 & -2.28013 \\
\hline 4.58210500 & 0.32384000 & -0.24269 \\
\hline 4.74961500 & -0.33416800 & -1.46489900 \\
\hline 6.01881100 & -0.70492500 & -1.90510300 \\
\hline 6.14894300 & -1.21982600 & -2.85230900 \\
\hline 5.67672200 & 0.64067400 & 0.56 \\
\hline 5.55481900 & 1.16613600 & 1.50 \\
\hline 6.94486600 & 0.26959300 & 0.11 \\
\hline 7.81051300 & 0.50651500 & 0.72 \\
\hline 7.11691700 & -0.39742100 & -1.10 \\
\hline 8.11408800 & -0.67640700 & -1.42803900 \\
\hline-1.45930600 & -3.71551000 & -1.09208 \\
\hline 3.54396000 & -4.29032800 & 1.20616300 \\
\hline 2.18868700 & -3.71845700 & -0.78781400 \\
\hline-1.59943200 & 0.24530200 & -3.05214000 \\
\hline-0.71676000 & 1.76674000 & -2.77920900 \\
\hline-1.05065500 & -4.73119800 & -1.15088000 \\
\hline .00423600 & -3.50418500 & -2.018218 \\
\hline 4440 & -3.68248400 & \\
\hline
\end{tabular}


2.77291900

2.92276600

3.40732300

0.75794900

0.56580500

1.76444100

1.29627900

2. 66320300

2.07537800

$-0.55909400$

$-1.30186100$

$-1.00257600$

$-0.36899400$

$-3.71826800$

$-4.64909900$

$-4.20388500$

$-5.99071400$

$-4.28196600$

$-5.54691600$

$-3.50011200$

$-6.43955500$

$-6.70612700$

$-5.92453700$

$-7.84359600$

$-8.21239700$

$-8.60809200$
1.20816200

2.29377500

0.80446300

1. 49185000

2. 55257400

1. 42248000

1.80264900

2.02536100

0.38841900

0.82825200

0.75045800

1.38994500

$-0.19256900$

$-0.46909700$

0.07253900

$-0.90282900$

0.18217100

0.39510100

$-0.79450300$

$-1.32149100$

$-0.25006000$

0.59492400

$-1.12258600$

$-0.13095800$

$-0.52143900$

0.35777400
1.27858200

1.23198200

2.06834000

2.82878600

2.62245700

3.99281400

4.90704300

3.81958700

4.19027000

3.26740500

2.46945500

4.09750000

3.62036400

0.39561300

1.31855400

$-0.86563900$

0.99983400

2.28844000

$-1.18480000$

$-1.57734800$

$-0.25446100$

1.70113900

$-2.14603900$

$-0.59239700$

$-1.70703800$

0.24916700 


\section{TS for acylation of alcohol $(S)$-4a with 4-nitrophenolate counter-ion}

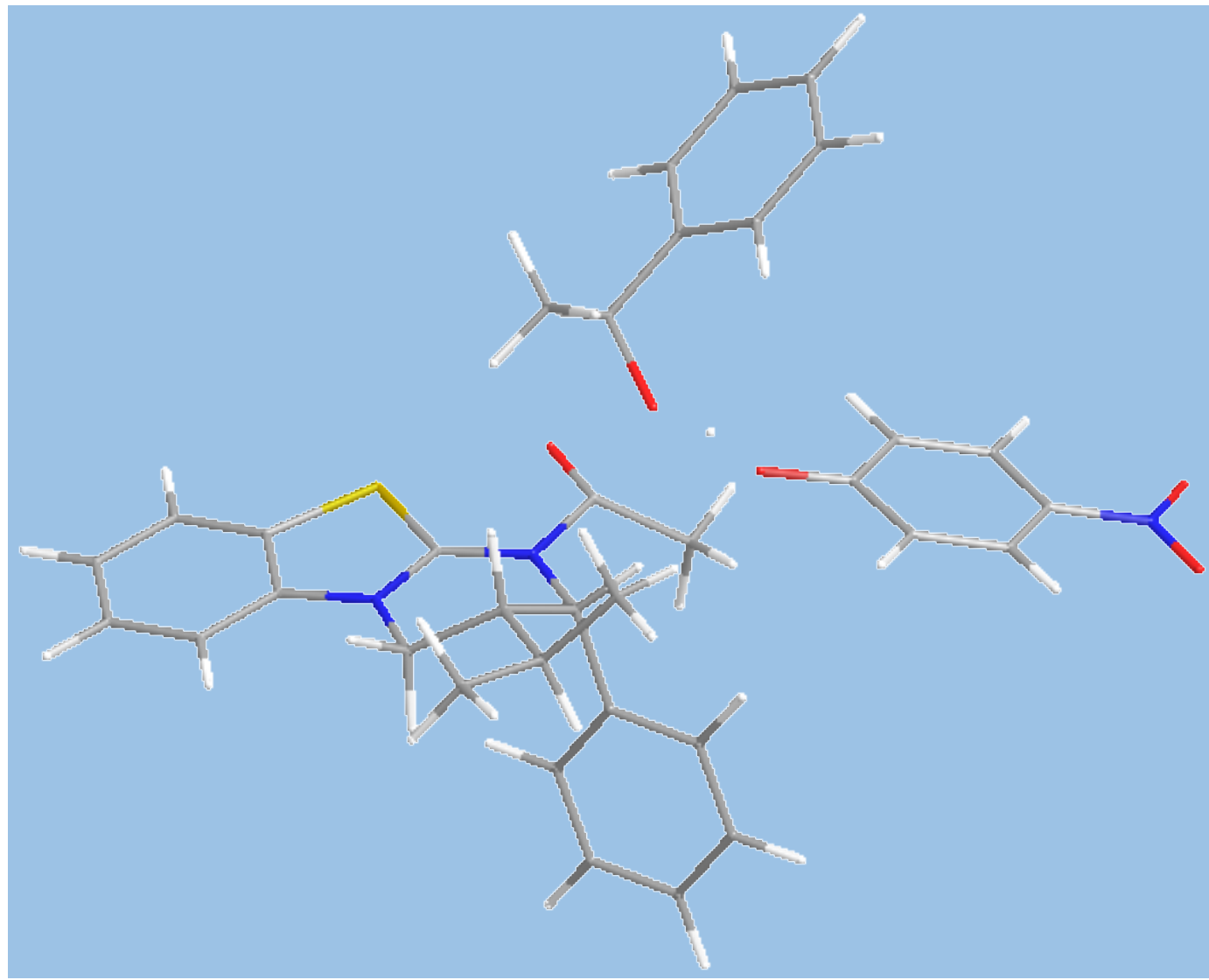

Zero-point correction=

Thermal correction to Energy=

Thermal correction to Enthalpy=

Thermal correction to Gibbs Free Energy=

Sum of electronic and zero-point Energies=

Sum of electronic and thermal Energies=

Sum of electronic and thermal Enthalpies=

Sum of electronic and thermal Free Energies=
0.657317 (Hartree/Particle)

0.697960

0.698904

0.578996

$-2293.816167$

$-2293.775524$

$-2293.774579$

$-2293.894488$

$E($ RB3LYP $)=-2295.13455547$

Number of imaginary frequencies: 1

Charge $=0$ Multiplicity $=1$

$\begin{array}{lrrr}\text { C } & -0.58692800 & 0.74339800 & -1.50065600 \\ \mathrm{C} & 0.52823300 & -0.04421300 & -2.14066900 \\ \mathrm{H} & 1.09206000 & -0.66404000 & -1.44724900 \\ \mathrm{O} & -1.07289200 & 1.73888200 & -2.02336200 \\ \mathrm{~N} & -1.51215600 & -0.03095100 & -0.62350900 \\ \mathrm{C} & -2.79016800 & 0.33962800 & -0.57173900 \\ \mathrm{~N} & -3.70757500 & -0.30391300 & 0.18892200 \\ \mathrm{C} & -1.86983100 & -1.21885100 & 1.52266200\end{array}$




$$
\begin{array}{r}
-1.01343400 \\
-0.93315700 \\
0.18381600 \\
0.29158200 \\
-0.71162900 \\
-1.81885700
\end{array}
$$$$
-1.92811800
$$$$
-2.78520700
$$$$
-2.59701400
$$$$
-0.62475600
$$$$
1.16802600
$$$$
0.98250900
$$$$
-0.00737400
$$$$
-1.75275600
$$$$
0.64161400
$$$$
0.35905700
$$$$
1.58893900
$$$$
2.73302900
$$$$
3.84621500
$$$$
3.83386300
$$$$
2.70211600
$$$$
1.59117400
$$$$
0.71408700
$$$$
2.68399800
$$$$
4.70215200
$$$$
-0.37408300
$$$$
1.41286600
$$$$
2.02576000
$$$$
-3.49184900
$$$$
-5.02351300
$$$$
-5.09232200
$$$$
-6.31175900
$$$$
-6.36513500
$$$$
-6.16999500
$$$$
-6.12557700
$$$$
-7.38833100
$$$$
-8.29306600
$$$$
-7.46112400
$$$$
-8.42084800
$$$$
-0.27995700
$$

4.72657900

2.75748900

1.20185500
$-1.15590200$

$-2.46204900$

$-3.28938500$

$-4.50825800$

$-4.91602800$

$-4.09357200$

$-2.87564900$

$-2.23904900$

$-4.39355700$

$-5.86122400$

$-5.13063200$

$-2.96631300$

$-0.85651300$

$-0.23917000$

1.41850600

2.72810500

3.63179200

3.33756300

4.17694800

5.32989600

5.63273800

4.78669200

5.02314500

6.52419100

5.98322600

3.14380100

0.71297800

$-0.13475400$

1.66667400

0.16425800

1.23998000

1.85259600

2.69116500

$-0.33618100$

$-1.18227100$

0.27875600

$-0.09472500$

1.36231000

1.82501400

2.71861500

3.92879300

2.43785600

0.67726800
0.22374400

$-0.55863000$

$-0.36833800$

$-1.03831600$

$-1.91895600$

$-2.12981800$

$-1.45588500$

$-1.65897600$

$-2.82639800$

$-2.44730500$

$-0.88171000$

0.29437900

0.51692700

2.00699400

0.02446000

0.46836300

0.42312800

1.17977300

1.14475100

0.35553000

$-0.40168700$

$-0.36710800$

$-0.96600600$

$-1.02396700$

0.32845400

$-0.24035500$

0.65578800

1.23618900

$-1.49265800$

0.03530400

$-0.85522800$

$-1.13736100$

$-1.82521600$

0.65509900

1.33223600

0.36720300

0.83687000

$-0.51720200$

$-0.72548700$

1.86889700

1.73216500

1.78893900

$-2.60292500$ 


\begin{tabular}{|c|c|c|}
\hline 0.09796200 & -0.68325800 & -2.92184200 \\
\hline 0.39677500 & 2.26720200 & 2.60311300 \\
\hline 1.20889400 & 2.13472300 & 1.85667000 \\
\hline 0.51958100 & 3.73444200 & 2.20456400 \\
\hline 3.34283100 & -1.35974900 & 1.14464800 \\
\hline 3.55854700 & -2.33982700 & 0.70281700 \\
\hline 3.98122500 & -1.22659300 & 2.01891200 \\
\hline 1.39700300 & -2.30096600 & 2.5340 \\
\hline 1.19230400 & -3.21989900 & 1.97075300 \\
\hline 2.46521700 & -2.63962000 & 3.59028200 \\
\hline 2.05031300 & -3.34587200 & 4.3171 \\
\hline 3.35868800 & -3.10838500 & 3.16209700 \\
\hline 2.77765000 & -1.746 & 4.14763900 \\
\hline 0.10593200 & -1.85853800 & 3.24481800 \\
\hline 0.67643000 & -1.51642200 & 2.56154000 \\
\hline 0.29564200 & -2.68457600 & 3.84288600 \\
\hline 0.31711200 & -1.02836500 & 3.93164500 \\
\hline 3.20348400 & -0.47212400 & 0.73739500 \\
\hline 3.90901600 & -1.56494200 & 1.30503400 \\
\hline 3.80132200 & 0.21435300 & -0.35237000 \\
\hline 5.13582000 & -1.96285000 & 0.80420100 \\
\hline 3.46429400 & -2.07939300 & 2.15204800 \\
\hline 5.02971900 & -0.18338500 & -0.85272800 \\
\hline 3.28144700 & 1.06418400 & -0.78110900 \\
\hline 5.69441400 & -1.27383100 & -0.27936000 \\
\hline 5.67768200 & -2.79742500 & 1.23308600 \\
\hline 49141000 & 0.33641200 & -1.68388300 \\
\hline 6.97502400 & -1.69454500 & -0.81085 \\
\hline 7.44621700 & -1.06766800 & -1.76773 \\
\hline .53670500 & -2.66375600 & -0 \\
\hline
\end{tabular}




\section{TS for acylation of alcohol $(R)-4 g$}

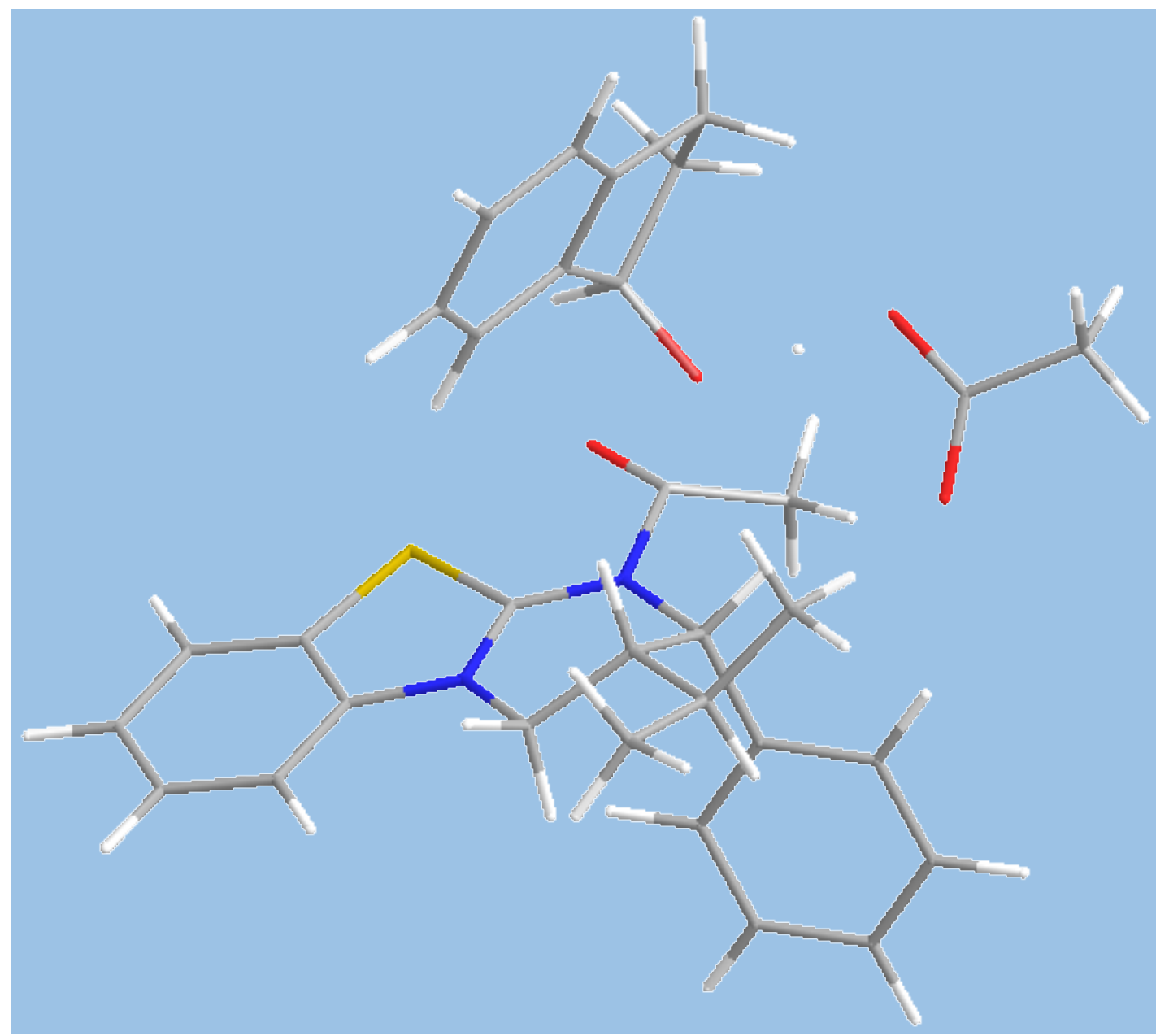

Zero-point correction=

Thermal correction to Energy=

Thermal correction to Enthalpy=

Thermal correction to Gibbs Free Energy=

Sum of electronic and zero-point Energies=

Sum of electronic and thermal Energies=

Sum of electronic and thermal Enthalpies=

Sum of electronic and thermal Free Energies=
0.620025 (Hartree/Particle)

0.656407

0.657351

0.549952

$-2049.079399$

$-2049.043017$

$-2049.042073$

$-2049.149473$

$E(R B 3 L Y P)=-2050.27586905$

Number of imaginary frequencies: 1

$\begin{array}{lccc}\text { Charge }=0 \text { Multiplicity }=1 & \\ \text { C } & -0.33162 & 0.29979 & -2.10978 \\ \mathrm{C} & -1.0808 & 1.53096 & -2.56197 \\ \mathrm{H} & -1.59702 & 2.06239 & -1.76409 \\ \mathrm{O} & 0.06052 & -0.55074 & -2.90911 \\ \mathrm{~N} & 0.54339 & 0.48607 & -0.91017 \\ \mathrm{C} & 1.61569 & -0.28334 & -0.78703 \\ & & & \mathrm{~S} 22\end{array}$




$$
\begin{aligned}
& 2.40264-0.27005 \quad 0.3173 \\
& \begin{array}{lll}
0.53554 & 0.75164 & 1.54579
\end{array} \\
& 0.150391 .41403 \quad 0.19182 \\
& \begin{array}{lll}
0.71677 & 2.81484 & -0.03197
\end{array} \\
& \begin{array}{lll}
-0.1053 & 3.92234 & 0.23039
\end{array} \\
& \begin{array}{lll}
0.38504 & 5.22062 & 0.08069
\end{array} \\
& \begin{array}{lll}
1.69615 & 5.43745 & -0.34498
\end{array} \\
& 2.51404 \quad 4.34398-0.63129 \\
& \begin{array}{lll}
2.02765 & 3.04469 & -0.477
\end{array} \\
& 2.67746 \quad 2.21226 \quad-0.73227 \\
& 3.53094 \quad 4.49861 \quad-0.98244 \\
& \begin{array}{lll}
2.07364 & 6.4492 & -0.4667
\end{array} \\
& \begin{array}{lll}
-0.26836 & 6.0648 & 0.28447
\end{array} \\
& \begin{array}{lll}
-1.14022 & 3.76062 & 0.51561
\end{array} \\
& \begin{array}{lll}
-0.93829 & 1.46477 & 0.15047
\end{array} \\
& 0.00025-0.20789 \quad 1.565 \\
& \begin{array}{lll}
-1.81606 & -0.49429 & -1.03449
\end{array} \\
& \begin{array}{lll}
-1.98741 & -1.8773-1.30195
\end{array} \\
& \begin{array}{lll}
-1.97524 & -2.68356 & -0.00877
\end{array} \\
& \begin{array}{lll}
-0.89118 & -3.01993 & 0.80075
\end{array} \\
& \begin{array}{lll}
-1.1117 & -3.73924 & 1.97977
\end{array} \\
& \begin{array}{lll}
-2.40959-4.11411 & 2.34202
\end{array} \\
& \begin{array}{lll}
-3.49638 & -3.78111 & 1.52744
\end{array} \\
& \begin{array}{lll}
-3.27432 & -3.06823 & 0.34926
\end{array} \\
& \begin{array}{lll}
-4.50245 & -4.08443 & 1.80969
\end{array} \\
& \begin{array}{lll}
-2.57317 & -4.67759 & 3.25746
\end{array} \\
& \begin{array}{lll}
-1.18111 & -2.17212-1.98808
\end{array} \\
& \begin{array}{lll}
-2.87963 & 0.12516 & -0.93964
\end{array} \\
& \begin{array}{lll}
-3.95317 & 0.64828 & -0.87157
\end{array} \\
& \begin{array}{lll}
-3.95825 & 1.80625 & -0.2904
\end{array} \\
& \begin{array}{lll}
-5.34431 & 2.40728 & -0.10918
\end{array} \\
& \begin{array}{lll}
-2.94757 & 2.41246 & 0.1025
\end{array} \\
& 2.21212-1.38876-2.02558 \\
& \begin{array}{lll}
3.55684 & -1.06346 & 0.2187
\end{array} \\
& 3.61971-1.74431-1.00149 \\
& 4.686-2.5952-1.28439 \\
& 4.73275-3.12931-2.22886 \\
& 4.56128-1.20324 \quad 1.17789 \\
& \begin{array}{lll}
4.52394 & -0.6644 & 2.11851
\end{array} \\
& \begin{array}{lll}
5.62903 & -2.05291 & 0.88707
\end{array} \\
& \begin{array}{lll}
6.42226 & -2.1751 & 1.61834
\end{array} \\
& \begin{array}{llll}
5.69255 & -2.74363 & -0.32918
\end{array} \\
& \begin{array}{llll}
6.53291 & -3.39991 & -0.53397
\end{array}
\end{aligned}
$$




$\begin{array}{rrc}-3.3774 & -2.23952 & -1.89796 \\ -5.92152 & 1.79184 & 0.5904 \\ -5.88365 & 2.40276 & -1.06189 \\ -5.27329 & 3.42588 & 0.27692 \\ -0.27207 & -4.01997 & 2.61147 \\ 0.12105 & -2.74548 & 0.50929 \\ -1.80789 & 1.20488 & -3.30756 \\ -0.36385 & 2.20614 & -3.04569 \\ -3.26087 & -3.11887 & -2.54356 \\ -3.78095 & -1.42572 & -2.50526 \\ 2.03317 & 0.44663 & 1.54705 \\ 2.63146 & 1.36285 & 1.62196 \\ 2.29579 & -0.20704 & 2.3805 \\ 0.10111 & 1.56307 & 2.79707 \\ 0.42255 & 2.60309 & 2.6525 \\ 0.76334 & 1.04726 & 4.08887 \\ -0.36566 & 1.59387 & 4.95067 \\ -1.85101 & 1.183 & 4.09831 \\ -1.54847 & -0.01729 & 4.25039 \\ -1.71271 & 1.54218 & 2.97028 \\ -1.77581 & 0.51903 & 3.16127 \\ -1.02338 & -3.36437 & -0.92975 \\ -1.91889 & -1.71313 & -0.33405\end{array}$




\section{TS for acylation of alcohol $(S)-4 g$}

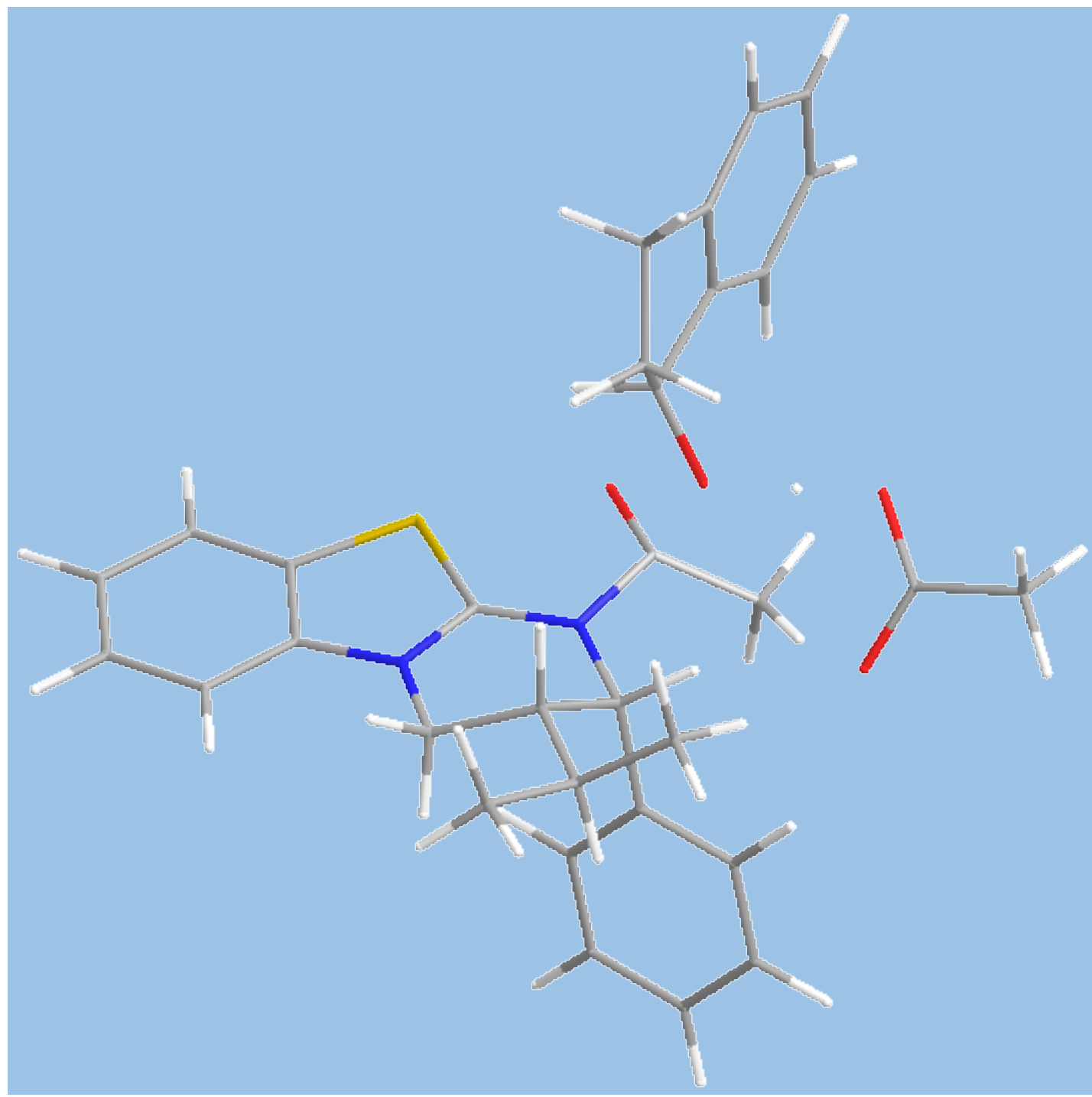

Zero-point correction=

Thermal correction to Energy=

Thermal correction to Enthalpy=

Thermal correction to Gibbs Free Energy=

Sum of electronic and zero-point Energies=

Sum of electronic and thermal Energies=

Sum of electronic and thermal Enthalpies=

Sum of electronic and thermal Free Energies=
0.619524 (Hartree/Particle)

0.656150

0.657094

0.548402

$-2049.078493$

$-2049.041867$

$-2049.040923$

$-2049.149614$

$E(R B 3 L Y P)=-2050.27512608$

Number of imaginary frequencies: 1

Charge $=0$ Multiplicity $=1$

$\begin{array}{llll}\text { C } & -0.50353 & -0.08889 & -1.60824 \\ \text { C } & -0.9021 & 1.18083 & -2.31867 \\ \text { H } & -0.97189 & 2.05396 & -1.6716\end{array}$




$$
\begin{aligned}
& \begin{array}{lll}
-0.70023 & -1.20143 & -2.08901
\end{array} \\
& \begin{array}{lll}
0.67561 & 0.01242 & -0.70695
\end{array} \\
& \begin{array}{lll}
1.3965 & -1.08042 & -0.48598
\end{array} \\
& \begin{array}{lll}
2.43224 & -1.10695 & 0.38771
\end{array} \\
& \begin{array}{lll}
1.51011 & 0.91513 & 1.43704
\end{array} \\
& \begin{array}{lll}
0.97554 & 1.28114 & 0.02313
\end{array} \\
& \begin{array}{lll}
1.88219 & 2.195 & -0.79756
\end{array} \\
& \begin{array}{lll}
1.58968 & 3.5675 & -0.84085
\end{array} \\
& \begin{array}{lll}
2.41585 & 4.44568 & -1.54416
\end{array} \\
& \begin{array}{lll}
3.53682 & 3.97032 & -2.22599
\end{array} \\
& \begin{array}{lll}
3.82251 & 2.60472 & -2.20941
\end{array} \\
& \begin{array}{lll}
3.00129 & 1.72516 & -1.50195
\end{array} \\
& \begin{array}{lll}
3.23103 & 0.66341 & -1.52803
\end{array} \\
& \begin{array}{lll}
4.68104 & 2.21885 & -2.75282
\end{array} \\
& \begin{array}{lll}
4.17467 & 4.65532 & -2.77809
\end{array} \\
& 2.17078 \quad 5.50413 \quad-1.56988 \\
& \begin{array}{llll}
0.69249 & 3.93561 & -0.3522
\end{array} \\
& \begin{array}{lll}
0.00721 & 1.76611 & 0.15598
\end{array} \\
& \begin{array}{lll}
0.71095 & 0.3258 & 1.90853
\end{array} \\
& \begin{array}{lll}
-1.81347 & 0.18367 & -0.04635
\end{array} \\
& \begin{array}{lll}
-2.36555 & -0.99807 & 0.47045
\end{array} \\
& \begin{array}{lll}
-3.71156 & -1.42889 & -0.10267
\end{array} \\
& \begin{array}{lll}
-4.13708 & -1.40672 & -1.42889
\end{array} \\
& \begin{array}{lll}
-5.4181 & -1.87312 & -1.73688
\end{array} \\
& \begin{array}{lll}
-6.25615 & -2.36255 & -0.72908
\end{array} \\
& \begin{array}{lll}
-5.82091 & -2.39674 & 0.59904
\end{array} \\
& \begin{array}{lll}
-4.54429 & -1.92749 & 0.90558
\end{array} \\
& \begin{array}{lll}
-6.47547 & -2.77725 & 1.3808
\end{array} \\
& \begin{array}{lll}
-7.25309 & -2.71696 & -0.98002
\end{array} \\
& \begin{array}{lll}
-1.64119 & -1.81101 & 0.28423
\end{array} \\
& \begin{array}{lll}
-2.57248 & 1.1606 & 0.04259
\end{array} \\
& \begin{array}{lll}
-3.3129 & 2.07701 & 0.19758
\end{array} \\
& \begin{array}{lll}
-2.71232 & 3.22043 & 0.26956
\end{array} \\
& \begin{array}{lll}
-3.63883 & 4.3934 & 0.55617
\end{array} \\
& \begin{array}{lll}
-1.48958 & 3.40175 & 0.12265
\end{array} \\
& 1.16786-2.62314 \quad-1.30816 \\
& \begin{array}{lll}
3.14793 & -2.31501 & 0.40444
\end{array} \\
& \begin{array}{lll}
2.59811 & -3.2591 & -0.46888
\end{array} \\
& \begin{array}{lll}
3.16033 & -4.52854 & -0.58638
\end{array} \\
& \begin{array}{lll}
2.73055 & -5.26447 & -1.25951
\end{array} \\
& \begin{array}{lll}
4.27847 & -2.60848 & 1.16888
\end{array} \\
& \begin{array}{lll}
4.71796 & -1.87356 & 1.83465
\end{array} \\
& \begin{array}{lll}
4.83986 & -3.87951 & 1.04364
\end{array}
\end{aligned}
$$




\begin{tabular}{|c|c|c|}
\hline 5.72056 & -4.12871 & 1.62769 \\
\hline .28804 & -4.83146 & 0.1776 \\
\hline .74164 & -5.81456 & 0.0966 \\
\hline .66964 & -0.90497 & 1.993 \\
\hline .11301 & 4.25785 & 1.534 \\
\hline 44237 & 4.42292 & -0.187 \\
\hline 0845 & 5.33385 & 0.543 \\
\hline 76798 & -1.85409 & -2.76 \\
\hline .47584 & -1.03918 & -2.20 \\
\hline .87447 & 0.99741 & -2.778 \\
\hline .16964 & 1.36958 & -3.111 \\
\hline .99267 & 0.12211 & 2.19 \\
\hline .78954 & -1.11083 & 2.61394 \\
\hline 73219 & 0.00775 & 1.29 \\
\hline 60084 & 0.55878 & $0.91^{7}$ \\
\hline 99831 & -0.43596 & 2.25874 \\
\hline 79261 & 2.14363 & 2.345 \\
\hline 38964 & 2.85898 & 1.76434 \\
\hline 6047 & 1.77224 & 3.600 \\
\hline 70746 & 2.65093 & 4.24 \\
\hline 61827 & 1.42463 & 3.36 \\
\hline 10046 & 0.9936 & 4.18 \\
\hline 48609 & 2.83273 & 2.773 \\
\hline 13629 & 3.1425 & 1.93028 \\
\hline 368 & 3.72343 & 3.370 \\
\hline 11315 & 2.15942 & 3.400 \\
\hline 842 & -1.88094 & 2.24 \\
\hline 49743 & -1.55808 & 3.06 \\
\hline .46824 & -2.87958 & 2. \\
\hline
\end{tabular}




\section{TS for acylation of alcohol $(R)-4 i$}

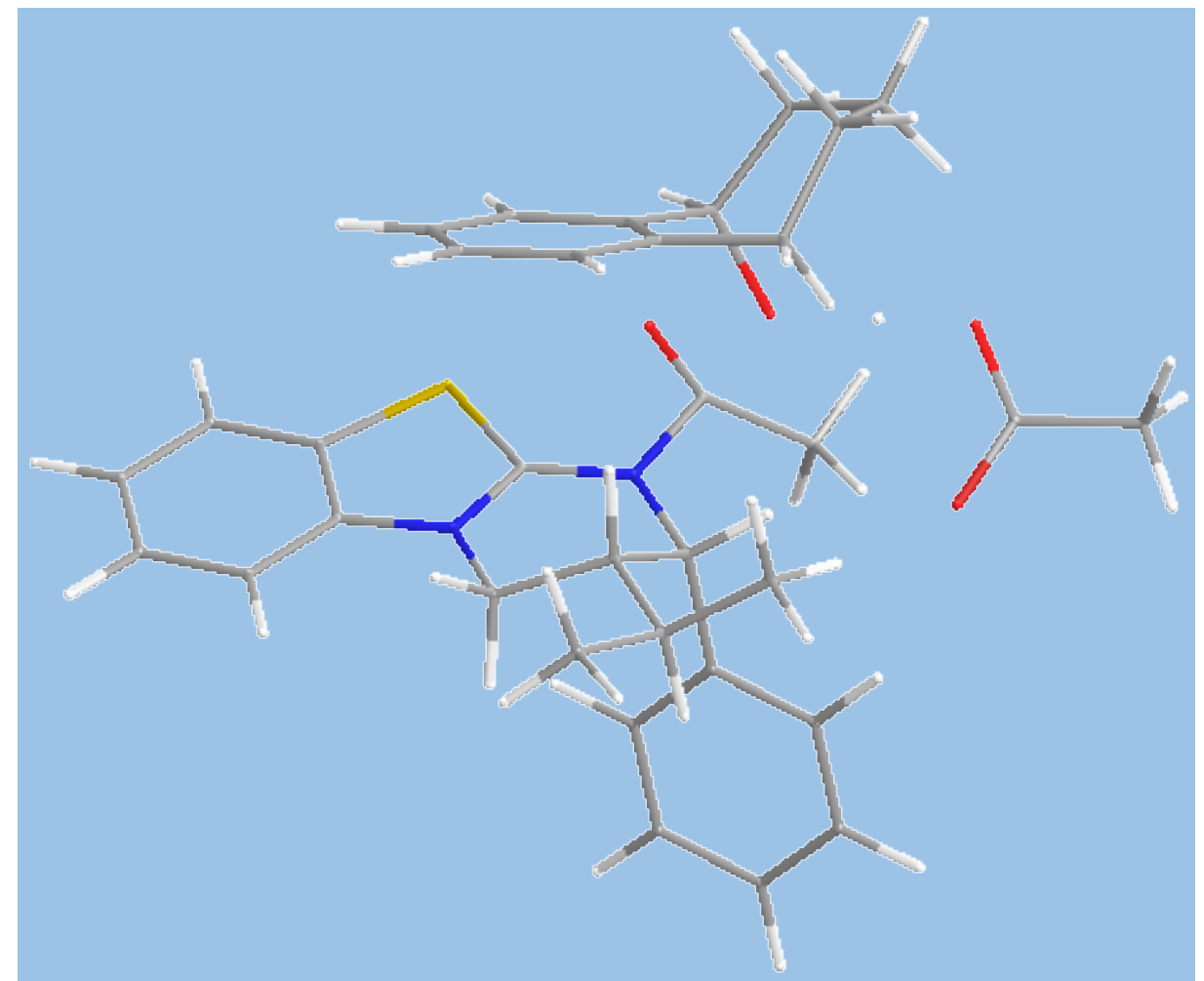

Zero-point correction=

Thermal correction to Energy=

Thermal correction to Enthalpy=

Thermal correction to Gibbs Free Energy=

Sum of electronic and zero-point Energies=

Sum of electronic and thermal Energies=

Sum of electronic and thermal Enthalpies=

Sum of electronic and thermal Free Energies=

$E(R B 3 L Y P)=-2128.92499703$

Number of imaginary frequencies: 1

Charge $=0$ Multiplicity $=1$

C

C

$\mathrm{H}$

O

N

C

$\mathrm{N}$

C

C

C

$$
\begin{array}{rrr}
-0.3924 & 0.3769 & -2.1317 \\
-1.3035 & 1.5099 & -2.5343 \\
-1.8389 & 1.9701 & -1.7071 \\
0.0912 & -0.3941 & -2.9571 \\
0.4451 & 0.6284 & -0.9266 \\
1.651 & 0.0784 & -0.8659 \\
2.4355 & 0.1601 & 0.2344 \\
0.4252 & 0.7317 & 1.5389 \\
-0.0854 & 1.4053 & 0.2335 \\
0.233 & 2.8941 & 0.1032
\end{array}
$$

0.679242 (Hartree/Particle)

0.717635

0.718579

0.607403

$-2127.646163$

$-2127.607771$

$-2127.606827$

$-2127.718002$ 


\begin{tabular}{|c|c|c|}
\hline 0.7647 & 3.8264 & 0.429 \\
\hline-0.5047 & 5.1964 & 0.367 \\
\hline 0.7483 & 5.6616 & -0.032 \\
\hline 1.74 & 4.7445 & -0.38 \\
\hline 1.4838 & 3.374 & -0.31 \\
\hline 2.2672 & 2.6844 & -0.61 \\
\hline 2.7146 & 5.0928 & -0.71 \\
\hline 0.9468 & 6.7287 & -0.086 \\
\hline 1.2923 & 5.9011 & 0.62 \\
\hline-1.7562 & 3.4736 & 0.69 \\
\hline-1.1658 & 1.2647 & 0.193 \\
\hline 0.059 & -0.3024 & 1.50 \\
\hline 1.83 & -0.675 & -1.06 \\
\hline-1.6382 & -2.0631 & -1.24 \\
\hline-0.941 & -2.6739 & -0.02 \\
\hline 0.3648 & -3.1589 & -0.17 \\
\hline 1.0869 & -3.6774 & 0.90 \\
\hline 0.4955 & -3.7163 & 2.16 \\
\hline 0.8155 & -3.2579 & 2.32 \\
\hline-1.5511 & -2.7436 & 1.24 \\
\hline-1.2894 & -3.3147 & 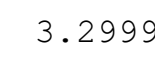 \\
\hline 1.0372 & -4 & \\
\hline 44 & -2.1 & -2 \\
\hline 08 & -0 & \\
\hline 318 & 0. & -1 \\
\hline-4.2175 & 1.3897 & -0 \\
\hline-5.6315 & 1.9453 & -0 \\
\hline-3.3525 & 1.9143 & 0.1 \\
\hline 2.4465 & -0.7575 & -2.19 \\
\hline 3.7288 & -0.3569 & 0.06 \\
\hline 3.9174 & -0.8882 & -1.21 \\
\hline 5.1363 & -1.4596 & -1.573 \\
\hline 5.2813 & -1.8791 & -2.565 \\
\hline 4.7555 & -0.3682 & 1.011 \\
\hline 4.6172 & 0.0553 & 1.99 \\
\hline 5.9762 & -0.9355 & 0.64 \\
\hline 6.7888 & -0.9536 & 1.36 \\
\hline 6.167 & -1.4768 & -0.63 \\
\hline 7.1255 & -1.9134 & -0.89 \\
\hline 2.9037 & -2.8408 & -1.68 \\
\hline 5.7053 & 2.8657 & -0.03 \\
\hline 5.3355 & 1.2044 & -0.21 \\
\hline .916 & 2.1347 & -1.65 \\
\hline
\end{tabular}


$\mathrm{H}$

$\begin{array}{rrr}2.0983 & -4.0483 & 0.7585 \\ 0.8234 & -3.1311 & -1.1573 \\ -2.0208 & 1.1156 & -3.2548 \\ -0.6862 & 2.2697 & -3.0307 \\ -2.5914 & -3.886 & -1.8306 \\ -3.1822 & -2.4648 & -2.6821 \\ 1.9529 & 0.6818 & 1.5218 \\ 2.3914 & 1.6736 & 1.6879 \\ 2.3241 & 0.0033 & 2.2918 \\ -0.116 & 1.3826 & 2.8406 \\ 0.0627 & 2.4645 & 2.7765 \\ 0.6112 & 0.8607 & 4.0947 \\ 0.1401 & 1.2716 & 4.9942 \\ 1.6687 & 1.147 & 4.1283 \\ -0.5494 & -0.2333 & 4.1612 \\ -1.6272 & 1.1451 & 3.0015 \\ -2.2156 & 1.4856 & 2.1461 \\ -1.9933 & 1.6691 & 3.8923 \\ -1.8306 & 0.0758 & 3.1388 \\ -4.1599 & -2.7966 & -0.8014 \\ -4.9126 & -3.4501 & -1.2651 \\ -4.578 & -1.7861 & -0.8298 \\ -3.9901 & -3.214 & 0.6655 \\ -3.6795 & -4.2661 & 0.737 \\ -3.9226 & -2.4089 & 2.5279\end{array}$




\section{TS for acylation of alcohol $(S)-4 i$}

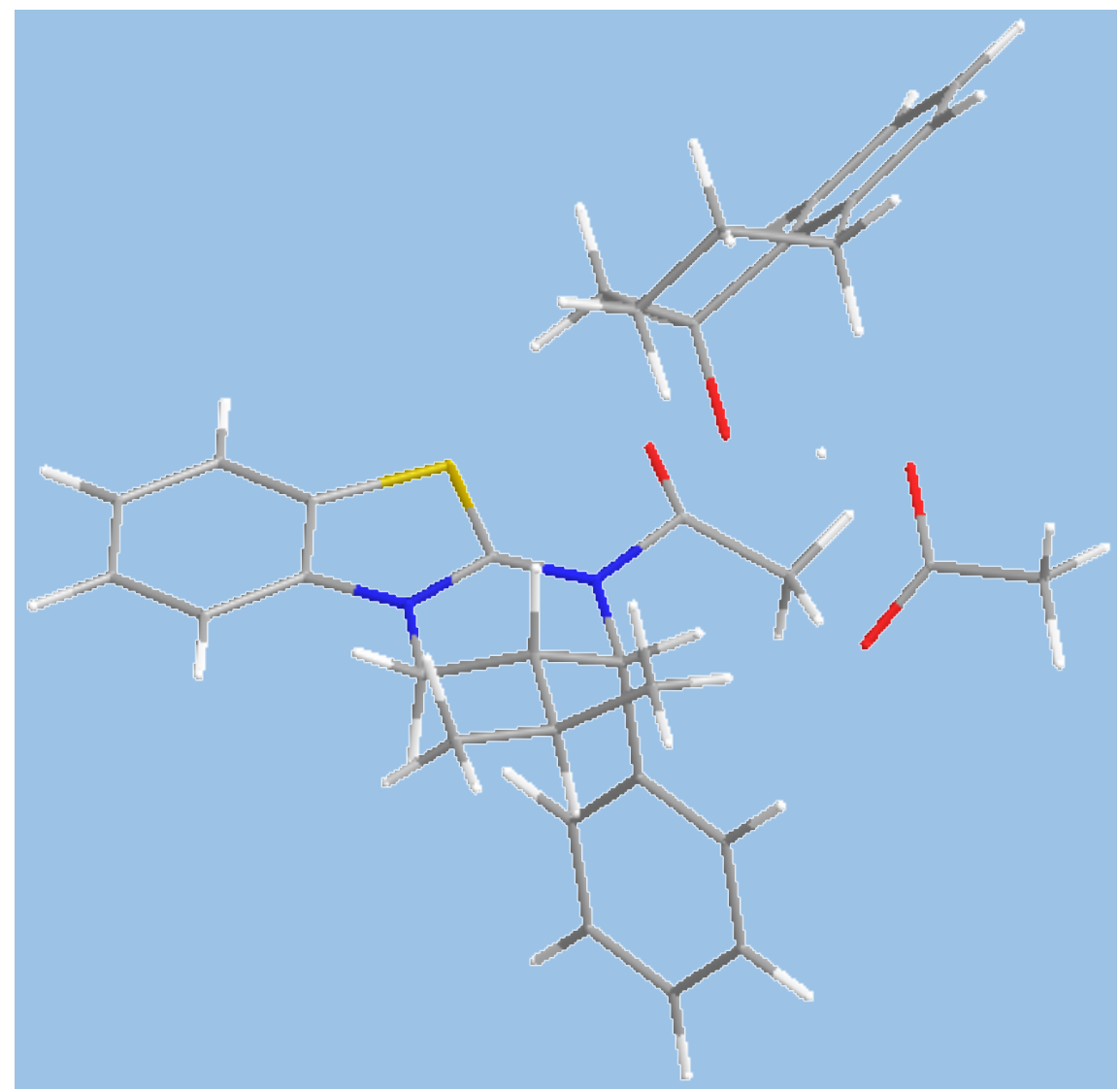

Zero-point correction=

Thermal correction to Energy=

Thermal correction to Enthalpy=

Thermal correction to Gibbs Free Energy=

Sum of electronic and zero-point Energies=

Sum of electronic and thermal Energies=

Sum of electronic and thermal Enthalpies=

Sum of electronic and thermal Free Energies=

$E(R B 3 L Y P)=-2128.92352085$

Number of imaginary frequencies: 1

$\begin{array}{lrrr}\mathrm{C} & -0.23475 & -0.18214 & -1.68003 \\ \mathrm{C} & -0.73733 & 1.03883 & -2.41027 \\ \mathrm{H} & -0.85727 & 1.92032 & -1.78392 \\ \mathrm{O} & -0.27828 & -1.30294 & -2.18893 \\ \mathrm{~N} & 0.91426 & 0.04092 & -0.75348 \\ \mathrm{C} & 1.75236 & -0.9631 & -0.53237 \\ \mathrm{~N} & 2.76522 & -0.88609 & 0.36621 \\ \mathrm{C} & 1.59234 & 0.99499 & 1.42257\end{array}$

0.678896 (Hartree/Particle)

0.717362

0.718306

0.606635

$-2127.644584$

$-2127.606118$

$-2127.605174$

$-2127.716846$ 
1.05568

1. 32204

$-0.00057$

1.88239

2.33955

$-0.78484$

1.46415

3.6797

$-0.79886$

2.21365

$4.64949-1.46681$

3.38352

$4.29914-2.14202$

3.7954

$2.96622-2.1543$

3.05035

1. 9955

3.37955

0.961

4.69381

2.6762

3.96194

5.0549

1.87074

5.6807

0.52981

3.9500

0.03931

1. 70307

$-0.31661$

0.85779

0.30573

0.11088

$-1.6064$

$-0.1378$

1.86195

$-2.12362$

$-1.44414$

$-0.17462$

$-3.60222$

$-1.5984$

0.02399

$-4.6929$

$-1.19954$

$-0.35181$

$-5.99227$

$-6.2388$

$-1.44823$

0.45455

$-5.1644$

$-2.05844$

$-0.01127$

$-3.8660$

$-2.43287$

$-1.24035$

$-3.0229$

$-2.2016$

$-2.04281$

$-5.3305$

$-2.48861$

$-1.59061$

$-7.26159$

$-2.9036$

$-2.21483$

$-1.59031$

$-2.2341$

$-3.00862$

$-2.43973$

$-2.09406$

$-1.56512$

$-3.23748$

0.8062

$-0.68553$

$-2.78541$

1.6655

$-0.2589$

$-3.86675$

2.8634

$-0.31285$

$-1.58985$

3.9319

$-0.10623$

1.7218

3.1622

$-0.06076$

3. 6154

$-2.50867$

0.04701

3.1973

$-2.00329$

$-1.38059$

3. 9051

$-2.98992$

0.38197

3.57787

$-4.18373$

$-0.51682$

4.75599

$-4.95303$

$-0.6392$

5.0932

$-2.17706$

$-1.3324$

5.46331

$-1.40754$

1.16787

1.85389

$6.35432-3.52809$

1.03786

$5.04396-4.36734$

1.63853

$5.60988-5.29007$

0.14617

$-1.74154-1.9812$ 


\begin{tabular}{|c|c|c|}
\hline-3.42038 & 4.92757 & -0.02451 \\
\hline-4.4924 & 3.78183 & 0.82666 \\
\hline-4.52096 & 3.84212 & -0.9336 \\
\hline-6.83359 & -1.14648 & 0.60936 \\
\hline-1.70072 & 0.7751 & -2.84909 \\
\hline-0.031 & 1.26144 & -3.22006 \\
\hline-0.64341 & -2.03886 & 1.44458 \\
\hline-2.10539 & -3.01587 & 1.50541 \\
\hline 2.91488 & 0.2392 & 1.30003 \\
\hline 3.72183 & 0.8936 & 0.94855 \\
\hline 3.20931 & -0.18767 & 2.26019 \\
\hline 1.7027 & 2.22975 & 2.35883 \\
\hline 2.21604 & 3.02645 & 1.80465 \\
\hline 2.5312 & 1.9369 & 3.62405 \\
\hline 2.51231 & 2.80857 & 4.28691 \\
\hline 3.58406 & 1.72201 & 3.40806 \\
\hline 2.11686 & 1.09028 & 4.18741 \\
\hline 0.31164 & 2.74002 & 2.772 \\
\hline-0.32977 & 2.98296 & 1.9212 \\
\hline 0.4128 & 3.6418 & 3.38717 \\
\hline-0.2073 & 1.98472 & 3.37711 \\
\hline-2.20837 & -1.16712 & 2.6313 \\
\hline-1.70461 & -1.55418 & 3.52868 \\
\hline-1.87357 & -0.128 & 2.50615 \\
\hline-3.72193 & -1.1816 & 2.87108 \\
\hline-4.071 & -2.21823 & 2.98472 \\
\hline-3.92764 & -0.68003 & 3.827 \\
\hline-4.54351 & -0.47359 & 1.77979 \\
\hline-4.10544 & 0.51402 & 1.58917 \\
\hline-5.55069 & -0.28814 & 2.17303 \\
\hline
\end{tabular}




\section{TS for acylation of alcohol $(\boldsymbol{R})-40$}

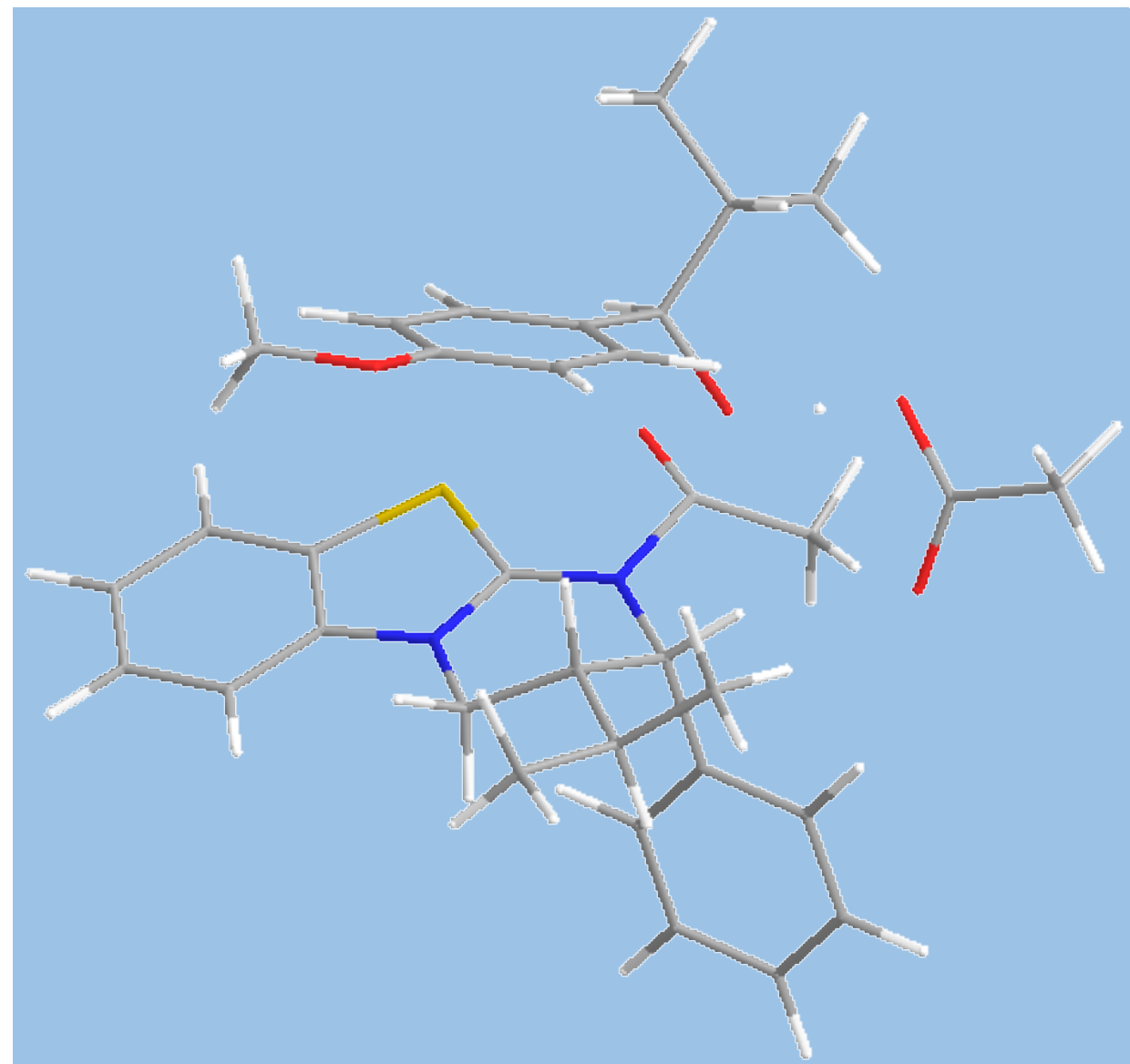

Zero-point correction=

Thermal correction to Energy=

Thermal correction to Enthalpy=

Thermal correction to Gibbs Free Energy=

Sum of electronic and zero-point Energies=

Sum of electronic and thermal Energies=

Sum of electronic and thermal Enthalpies=

Sum of electronic and thermal Free Energies=
0.701960 (Hartree/Particle)

0.744100

0.745044

0.624859

$-2204.040916$

$-2203.998775$

$-2203.997831$

$-2204.118016$

$E(R B 3 L Y P)=-2205.37662273$

Number of imaginary frequencies: 1

$\begin{array}{lrrr}\text { Charge }=0 \text { Multiplicity }=1 & \\ \text { C } & -0.99705900 & 0.58654500 & -2.10407100 \\ \mathrm{C} & -2.38297900 & 1.16561900 & -2.24620500 \\ \mathrm{H} & -2.98875400 & 1.10389900 & -1.34308900 \\ \mathrm{O} & -0.32712600 & 0.24475100 & -3.07667300 \\ \mathrm{~N} & -0.20658000 & 1.07003400 & -0.93484600 \\ \mathrm{C} & 1.11687400 & 1.06898200 & -1.02490500 \\ \mathrm{~N} & 1.92084500 & 1.39659900 & 0.01528700\end{array}$


0.02592100

$-0.87810600$

$-1.26626600$

$-2.53304200$

$-2.91228200$

$-2.04230300$

$-0.79004400$

$-0.40659700$

0.56321900

$-0.11044200$

$-2.34209600$

$-3.89896500$

$-3.23186200$

$-1.78522500$

0.13664600

$-1.59862400$

$-0.81670100$

$-0.02109400$

1.36483100

2.10085300

1.43511600

0.04099400

$-0.66982900$

$-1.74710600$

$-0.46032700$

$-0.09815500$

$-2.74889200$

$-3.79887500$

$-4.41766200$

$-5.76895000$

$-3.98476800$

2.02740600

3.28734000

3.52993100

4.82888300

5.01880300

4.33133500

4.14562200

5.62957300

6.45634600

5.87881000

6.89728400

$-1.66786200$
0.97381000

1.43805000

2. 91490000

3.27173700

4.61298200

5.61894100

5.27383600

3. 93355000

3.69128200

6.04617400

6.66210800

4.86860200

2.49202800

0.83367900

$-0.11181000$

$-1.09966000$

$-2.22360500$

$-2.69902900$

$-2.85771300$

$-3.27956900$

$-3.55141400$

$-3.39539000$

$-2.97466200$

$-2.84797800$

$-3.61720900$

$-1.89989100$

$-1.35863900$

$-1.68785900$

$-0.77300500$

$-1.21519700$

0.36847100

0.70623000

1.45107100

1.10867000

1.09432900

0.82368000

1.80063200

2.08230800

1.78879800

2.05936900

1.43854900

1.43673800

$-3.35600300$
1.52562200

0.34753800

0.36702000

0.85536600

0.93032300

0.50799600

$-0.00144100$

$-0.07196200$

$-0.49769100$

$-0.35207200$

0.56148200

1.30739400

1.14240900

0.37378800

1.40240500

$-1.14905800$

$-1.48962000$

$-0.27580700$

$-0.32018800$

0.79573100

1.99353400

2. 05917700

0.94216200

1.00839100

2.99698200

$-2.26220000$

$-0.71354200$

$-0.30579200$

0.37898500

0.91884900

0.59977700

$-2.49114500$

$-0.29979500$

$-1.63401900$

$-2.13761300$

$-3.17214700$

0.55840600

1.58923800

0.04748700

0.69727500

$-1.28506800$

$-1.66139800$

$-2.13518300$ 


\begin{tabular}{|c|c|c|}
\hline-6.26469700 & -0.38964700 & 1.43276700 \\
\hline-5.63320900 & -2.05249100 & 1.61256400 \\
\hline-6.39780900 & -1.57717900 & 0.09876800 \\
\hline 3.17662100 & -3.39094600 & 0.71406600 \\
\hline 1.89425500 & -2.65310900 & -1.2487 \\
\hline-2.87233300 & 0.61217400 & -3.04921000 \\
\hline-2.28779000 & 2.21498700 & -2.552 \\
\hline-2.36269500 & -3.73029300 & -1.37 \\
\hline 1.41287300 & 1.59865300 & 1.379 \\
\hline 1.39723300 & 2.67308800 & 1.60 \\
\hline 2.12462400 & 1.11720700 & 2.05 \\
\hline-0.57790900 & 1.23638600 & 2.93 \\
\hline-0.89286200 & 2.28777600 & 2.97 \\
\hline 0.45080200 & 1.01164400 & $4.05^{\circ}$ \\
\hline-0.03776000 & 1.12070500 & 5.03 \\
\hline 1.27824200 & 1.73013400 & 4.033 \\
\hline 0.87251200 & -0.00096100 & 4.014 \\
\hline-1.80559900 & 0.34743900 & 3.191 \\
\hline-2.59388000 & 0.47102200 & 2.445 \\
\hline-2.23256000 & 0.57970700 & 4.1744 \\
\hline-1.51400800 & -0.71034400 & 3.19627100 \\
\hline 2.04221800 & -3.96594300 & 3.15005200 \\
\hline 3.43923500 & -4.19504100 & 3.12222500 \\
\hline 3.70830200 & -4.54137000 & 4.12226100 \\
\hline 3.70950800 & -4.96527700 & 2.38717000 \\
\hline 3.99873500 & -3.27633500 & 2.89631200 \\
\hline-0.78438200 & -4.52487400 & -2.59663000 \\
\hline-0.06577700 & -4.19598700 & -3.35981900 \\
\hline-0.21689400 & -4.96361800 & -1.76951900 \\
\hline-1.39674600 & -5.31775300 & -3.0423 \\
\hline-2.49736400 & -2.81035300 & -3.3063 \\
\hline-3.05205600 & -3.62054800 & -3.7956 \\
\hline-3.21968800 & -2.06496000 & -2.9657 \\
\hline 35035100 & 012400 & -4.0 \\
\hline
\end{tabular}




\section{TS for acylation of alcohol $(S)-40$}

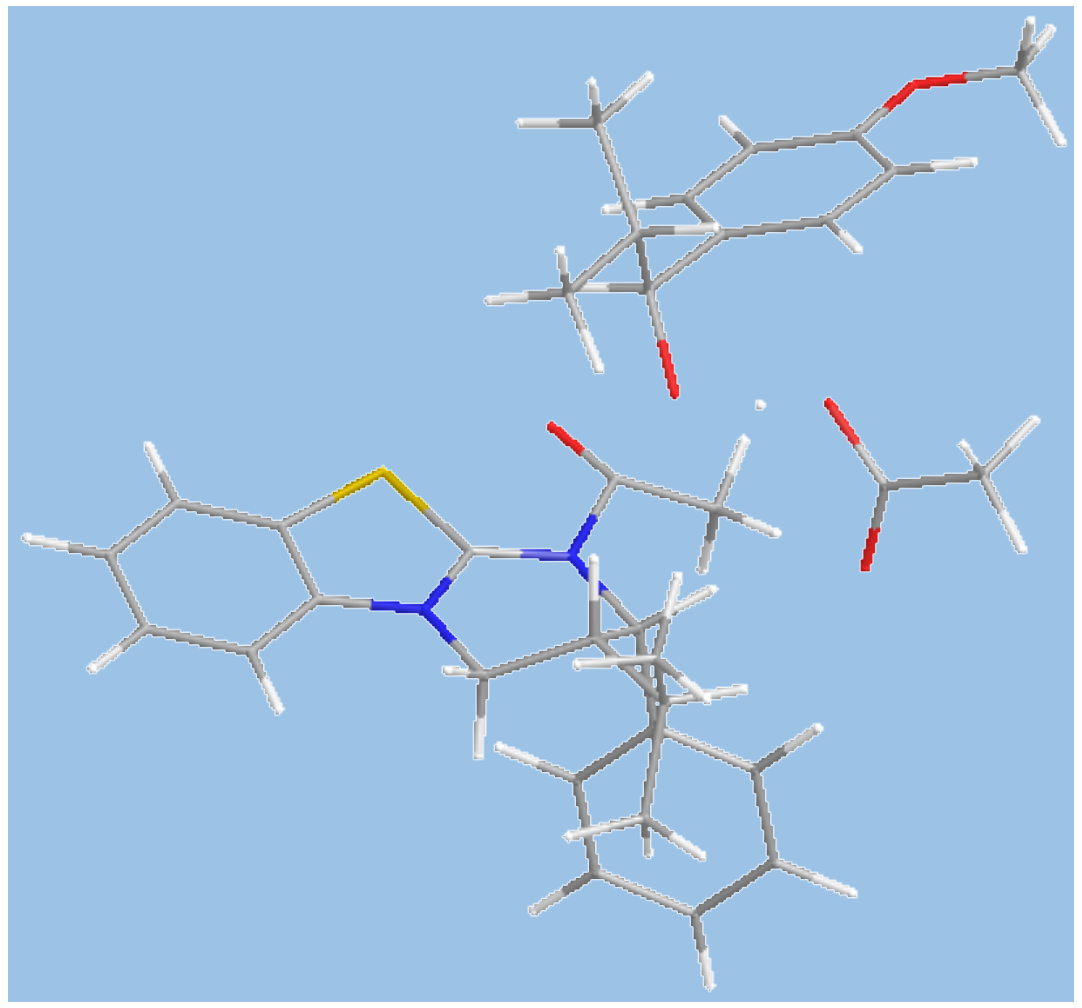

Zero-point correction=

Thermal correction to Energy=

Thermal correction to Enthalpy=

Thermal correction to Gibbs Free Energy=

Sum of electronic and zero-point Energies=

Sum of electronic and thermal Energies=

Sum of electronic and thermal Enthalpies=

Sum of electronic and thermal Free Energies=
0.702128 (Hartree/Particle)

0.744456

0.745400

0.623031

$-2204.035231$

$-2203.992903$

$-2203.991959$

$-2204.114328$

$E(R B 3 L Y P)=-2205.37202076$

Number of imaginary frequencies: 1

$\begin{array}{lrrr}\text { Charge }=0 \text { Multiplicity }=1 & \\ \text { C } & -0.39049400 & -0.29298400 & -1.24592500 \\ \text { C } & -1.09879000 & 0.89567300 & -1.85473300 \\ \text { H } & -1.13375900 & 1.77274900 & -1.21034900 \\ \text { O } & -0.54717100 & -1.43429500 & -1.68611000 \\ \text { N } & 0.97424700 & -0.02466700 & -0.69610500 \\ \text { C } & 1.80157800 & -1.04880400 & -0.53740300 \\ \text { N } & 3.00041300 & -0.93758700 & 0.09001200 \\ \text { C } & 2.18897600 & 1.13388500 & 1.10020700 \\ \text { C } & 1.33472900 & 1.32280800 & -0.17834100 \\ \text { C } & 1.92787000 & 2.20344700 & -1.27214100\end{array}$


1.51957900

2.06418100

3.01318500

3.40856200

2.86814200

3.17110300

4.13304700

3.43179500

1.73527300

0.76328500

0.40135800

1.54728100

$-1.26827500$

$-2.11317100$

$-3.49394600$

$-4.38171000$

$-5.63947200$

$-6.03346200$

$-5.15568900$

$-3.90756100$

$-3.22894000$

$-5.47019500$

$-1.64646700$

$-1.64527200$

$-2.01920700$

$-1.67145800$

$-2.31525200$

$-0.89986600$

1.50434700

3.77499400

3.11033400

3.71031900

3.19316000

5.05648800

5.58175300

5.65346100

6.65035500

4.98928300

5.47304800

$-2.18446500$

$-1.89486500$

$-2.17176000$

$-3.39569700$
3.54455700

4.39947900

3.92800800

2.59062000

1.73487100

0.69121000

2.20794100

4.59350400

5.43438900

3.90584200

1.77729900

0.53211000

$-0.20552900$

$-1.32867500$

$-1.12365000$

$-0.14292000$

0.03841500

$-0.77307200$

$-1.75360900$

$-1.91833900$

$-2.67080500$

$-2.37008200$

$-2.17134600$

0.83183600

1.60218800

2.83792300

3.83419600

3.20857400

$-2.67752600$

$-2.10824400$

$-3.16482800$

$-4.41808300$

$-5.24088600$

$-2.27193500$

$-1.45075200$

$-3.52816500$

$-3.67729500$

$-4.59198500$

$-5.56122700$

$-1.73107200$

4.83014000

3.51525800

3.86501600
$-1.34479200$

$-2.30396500$

$-3.21278800$

$-3.16278600$

$-2.20126500$

$-2.19852200$

$-3.87683800$

$-3.96294200$

$-2.34757200$

$-0.65389400$

0.15589500

1.75681700

0.53886600

0.71975800

0.09299500

0.55098000

$-0.03132600$

$-1.10134900$

$-1.57955300$

$-0.98762900$

$-1.38073800$

$-2.41654900$

0.18641900

1.26333100

2.01027400

1.78285700

2.73272100

0.88965900

$-1.14136500$

0.07531900

$-0.55549400$

$-0.65861800$

$-1.14355800$

0.60415200

1.07995800

0.49447400

0.89812400

$-0.12811900$

$-0.20196600$

2.22035900

2.58252800

3.77002300

2. 55166400 


\begin{tabular}{|c|c|c|}
\hline-6.29613500 & 0.81058800 & 0.35472200 \\
\hline-4.08702700 & 0.50810700 & 1.36881800 \\
\hline 2.11808200 & 0.57068000 & -2.06801200 \\
\hline 0.60548800 & 1.15312100 & -2.80061000 \\
\hline 2.70984300 & -0.93064900 & 2.75932500 \\
\hline 3.41968500 & 0.28946300 & 0.78438700 \\
\hline 4.13269100 & 0.82761300 & 0.15 \\
\hline 3.93258400 & -0.01417800 & 1.70 \\
\hline 2.51035000 & 2.44901200 & 1.86 \\
\hline 1.66013400 & 3.11841100 & 1.69 \\
\hline 3.79079700 & 3.17473300 & 1.42 \\
\hline 3.86764400 & 4.13785900 & 1.93 \\
\hline 3.80627900 & 3.37458800 & 0.34 \\
\hline 4.69240100 & 2.60305200 & 1.67 \\
\hline 2.56285000 & 2.17612800 & 3.383 \\
\hline 1.61916900 & 1.75406400 & 3.74667000 \\
\hline 2.75038200 & 3.10373900 & 3.93606600 \\
\hline 3.36752200 & 1.47493100 & 3.64294600 \\
\hline-7.24044700 & -0.68503700 & -1.74532600 \\
\hline-8.16082400 & 0.29488700 & -1.30196000 \\
\hline-7.75536700 & 1.31087100 & -1.40543800 \\
\hline-9.04080100 & 0.19312200 & -1.94081500 \\
\hline-8.45426000 & 0.13409300 & -0.25537300 \\
\hline 0.77998100 & -1.85771700 & 2.82470200 \\
\hline 0.23928300 & -0.91033600 & 2.75547800 \\
\hline 0.83458300 & -2.14823500 & 3.88124100 \\
\hline-0.19472900 & -2.62497400 & 2.29877100 \\
\hline-2.97364800 & -3.03543700 & 2.40861200 \\
\hline 3.99488700 & -2.95208300 & 2.02437 \\
\hline .48577400 & -3.86757300 & 1.8829 \\
\hline 14000 & -3.30425000 & 3 \\
\hline
\end{tabular}




\section{TS for acylation of alcohol $(R)-4 q$}



Zero-point correction=

Thermal correction to Energy=

Thermal correction to Enthalpy=

Thermal correction to Gibbs Free Energy=

Sum of electronic and zero-point Energies=

Sum of electronic and thermal Energies=

Sum of electronic and thermal Enthalpies=

Sum of electronic and thermal Free Energies=
0.611317 (Hartree/Particle)

0.649919

0.650864

0.537253

$-2103.229003$

$-2103.190401$

$-2103.189457$

$-2103.303068$

$E(R B 3 L Y P)=-2104.43717661$

Number of imaginary frequencies: 1

$\begin{array}{lccc}\text { Charge }=0 \text { Multiplicity }=1 & & \\ \text { C } & 0.94611 & -0.41505 & -2.21247 \\ \mathrm{C} & 2.4315 & -0.53511 & -2.43934 \\ \mathrm{H} & 3.04309 & -0.17029 & -1.61575 \\ \mathrm{O} & 0.13957 & -0.40863 & -3.13797 \\ \mathrm{~N} & 0.42989 & -0.92879 & -0.92149 \\ \mathrm{C} & -0.83914 & -1.32035 & -0.85817\end{array}$




$$
\begin{aligned}
& \begin{array}{llll}
-1.42246 & -1.72286 & 0.29538
\end{array} \\
& 0.36772-0.57514 \quad 1.52692 \\
& \begin{array}{lll}
1.27819 & -0.90207 & 0.30838
\end{array} \\
& \begin{array}{lll}
2.10598 & -2.17829 & 0.43781
\end{array} \\
& \begin{array}{lll}
3.45219 & -2.07233 & 0.82108
\end{array} \\
& \begin{array}{lll}
4.23286 & -3.21677 & 0.99163
\end{array} \\
& \begin{array}{lll}
3.68958 & -4.48295 & 0.77048
\end{array} \\
& 2.35908-4.59823 \quad 0.36638 \\
& \begin{array}{lll}
1.57502 & -3.45538 & 0.20065
\end{array} \\
& 0.55008-3.57186-0.14084 \\
& \begin{array}{lll}
1.92937 & -5.5774 & 0.1722
\end{array} \\
& \begin{array}{lll}
4.30093 & -5.3722 & 0.89792
\end{array} \\
& \begin{array}{lll}
5.27423 & -3.11415 & 1.28477
\end{array} \\
& 3.89326-1.08881 \quad 0.95125 \\
& \begin{array}{lll}
1.95051 & -0.0553 \quad 0.16832
\end{array} \\
& \begin{array}{lll}
-0.08261 & 0.402 & 1.30403
\end{array} \\
& 1.05525 \quad 1.54849-1.49079 \\
& \begin{array}{lll}
0.01337 & 2.32594 & -2.01085
\end{array} \\
& \begin{array}{lll}
-0.90227 & 2.83835 & -0.89927
\end{array} \\
& \begin{array}{lll}
-2.29392 & 2.86591 & -1.05933
\end{array} \\
& \begin{array}{lll}
-3.12864 & 3.3365 & -0.04904
\end{array} \\
& \begin{array}{lll}
-2.57349 & 3.79468 & 1.15697
\end{array} \\
& \begin{array}{lll}
-1.17662 & 3.77917 & 1.32687
\end{array} \\
& \begin{array}{lll}
-0.35818 & 3.30236 & 0.30897
\end{array} \\
& \begin{array}{lll}
0.71958 & 3.28372 & 0.43854
\end{array} \\
& \begin{array}{lll}
-0.74796 & 4.14231 & 2.25627
\end{array} \\
& \begin{array}{lll}
-0.59431 & 1.68462 & -2.67215
\end{array} \\
& \begin{array}{lll}
2.15359 & 2.19814 & -1.25037
\end{array} \\
& 3.0617 \quad 2.84991-1.0123 \\
& \begin{array}{llll}
3.96868 & 2.25371 & -0.29299
\end{array} \\
& \begin{array}{lll}
5.16535 & 3.13125 & 0.03314
\end{array} \\
& \begin{array}{lll}
3.90203 & 1.08286 & 0.10767
\end{array} \\
& \begin{array}{lll}
-1.93051 & -1.4284 & -2.2373
\end{array} \\
& \begin{array}{lll}
-2.73414 & -2.20287 & 0.14832
\end{array} \\
& \begin{array}{lll}
-3.17386 & -2.12141 & -1.17657
\end{array} \\
& \begin{array}{lll}
-4.45603 & -2.53991 & -1.5265
\end{array} \\
& \begin{array}{lll}
-4.80023 & -2.47214 & -2.55428
\end{array} \\
& \begin{array}{lll}
-3.55644 & -2.71565 & 1.15313
\end{array} \\
& \begin{array}{lll}
-3.21497 & -2.79436 & 2.17952
\end{array} \\
& \begin{array}{lll}
-4.83768 & -3.13636 & 0.79682
\end{array} \\
& \begin{array}{lll}
-5.49416 & -3.53849 & 1.56232
\end{array} \\
& \begin{array}{lll}
-5.28563 & -3.04952-0.52719
\end{array} \\
& \begin{array}{llll}
-6.2873 & -3.38277 & -0.78075
\end{array}
\end{aligned}
$$




\begin{tabular}{|c|c|c|}
\hline 0.53161 & 3.4955 & -2.87306 \\
\hline 4.83802 & 4.00789 & 0.6028 \\
\hline 5.61771 & 3.50155 & -0.89288 \\
\hline 5.90371 & 2.57124 & 06 \\
\hline .20633 & 3.34682 & -0 \\
\hline .73049 & 2.50773 & -1.9 \\
\hline .65787 & 0.03898 & -3.3 \\
\hline .66616 & -1.59037 & -2 \\
\hline .29879 & 4.06569 & -3.3 \\
\hline .1446 & 3.09801 & -3 \\
\hline .15019 & 4.17755 & -2 \\
\hline .76569 & -1.59689 & 1 . \\
\hline .40077 & -2.58212 & 1 . \\
\hline .53249 & -1.27053 & 2 . \\
\hline 148 & -0.45364 & 2 . \\
\hline 1.77646 & -1.33644 & 2 . \\
\hline 0.18091 & -0.42938 & \\
\hline .75588 & -0.25675 & \\
\hline 0.36344 & -1.37051 & 4 \\
\hline .55329 & 0.38266 & 4 \\
\hline 74 & 0.80286 & 2 \\
\hline 627 & 0.84416 & 2 \\
\hline 883 & 0.8412 & 3 \\
\hline 734 & 1.70739 & 9 \\
\hline 419 & 4.26228 & 2.211 \\
\hline 56 & .63293 & \\
\hline
\end{tabular}




\section{TS for acylation of alcohol $(S)-4 q$}

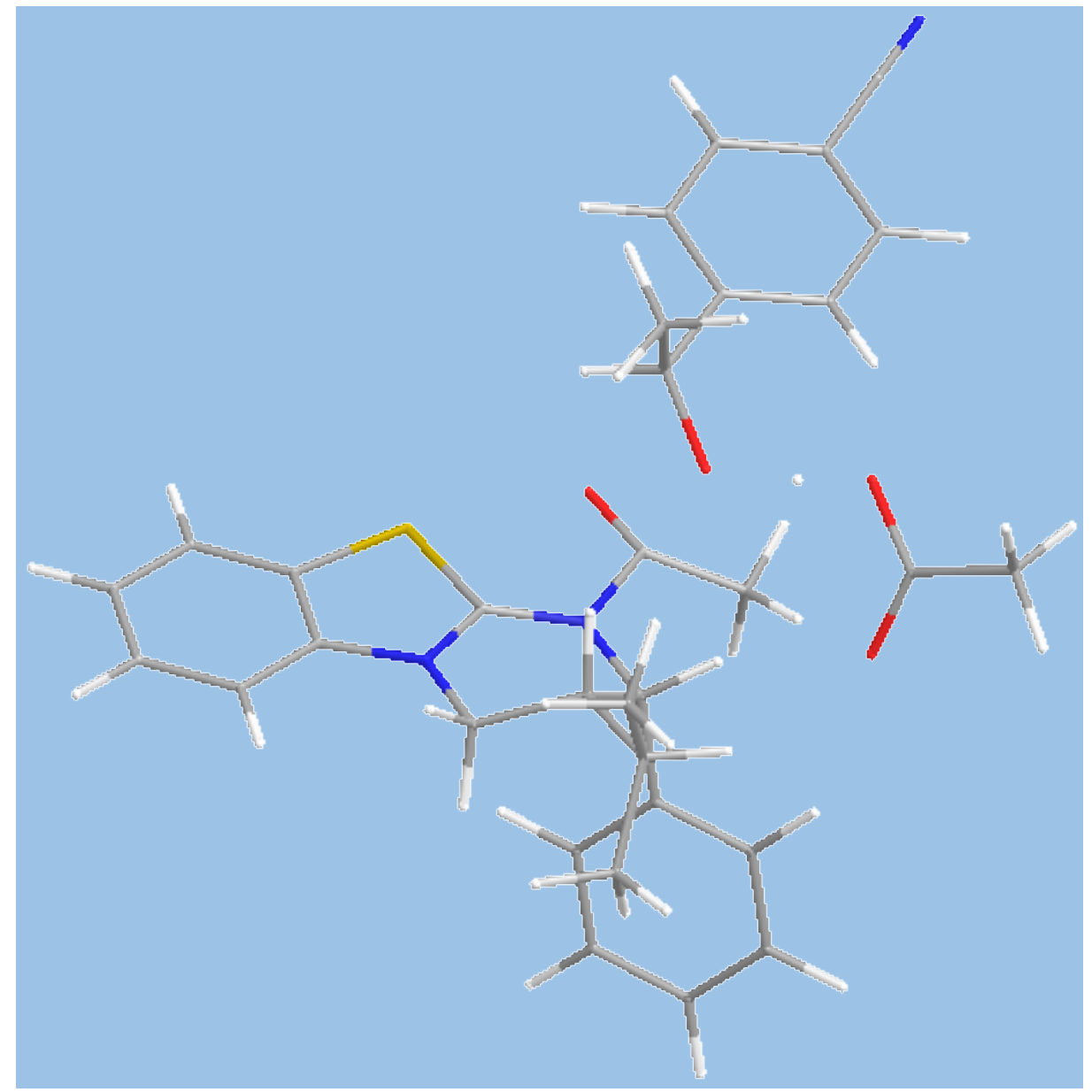

Zero-point correction=

Thermal correction to Energy=

Thermal correction to Enthalpy=

Thermal correction to Gibbs Free Energy=

Sum of electronic and zero-point Energies=

Sum of electronic and thermal Energies=

Sum of electronic and thermal Enthalpies=

Sum of electronic and thermal Free Energies=

$E(R B 3 L Y P)=-2104.43303333$

Number of imaginary frequencies: 1
0.611885 (Hartree/Particle)

0.650671

0.651615

0.536451

$-2103.223911$

$-2103.185124$

$-2103.184180$

$-2103.299344$

Charge $=0$ Multiplicity $=1$

$\begin{array}{lrrr}\mathrm{C} & -0.62613 & 0.22436 & 1.21776 \\ \mathrm{C} & -1.09417 & -0.99425 & 1.97526 \\ \mathrm{H} & -0.95839 & -1.93292 & 1.44109 \\ \mathrm{O} & -1.02417 & 1.35461 & 1.48575 \\ \mathrm{~N} & 0.7358 & 0.15558 & 0.63308 \\ \mathrm{C} & 1.35767 & 1.29228 & 0.33779\end{array}$


$2.52664 \quad 1.33277-0.34857$

$2.04568-0.93197-1.13502$

$\begin{array}{lll}1.31801 & -1.15079 & 0.21444\end{array}$

$2.12361-1.7895 \quad 1.33962$

$\begin{array}{lll}1.98441 & -3.16873 & 1.56166\end{array}$

$2.72873-3.80444 \quad 2.55641$

$3.61233-3.07429 \quad 3.35312$

$3.74162-1.69916 \quad 3.15432$

$3.00155-1.06208 \quad 2.15673$

$\begin{array}{lll}3.10067 & 0.01361 & 2.03819\end{array}$

$4.41343-1.11735 \quad 3.77981$

$\begin{array}{lll}4.18642 & -3.56993 & 4.13124\end{array}$

$2.60728-4.87257 \quad 2.71573$

$\begin{array}{lll}1.28102 & -3.73454 & 0.95771\end{array}$

$0.4698 \quad-1.79457-0.01615$

$1.26039-0.52046-1.78275$

$\begin{array}{lll}-1.50947 & -0.34941 & -0.57608\end{array}$

$\begin{array}{lll}-2.25367 & 0.71438 & -1.09637\end{array}$

$\begin{array}{lll}-3.66507 & 0.82749 & -0.50978\end{array}$

$\begin{array}{lll}-4.40368 & -0.30651 & -0.14399\end{array}$

$\begin{array}{lll}-5.69716 & -0.1875 & 0.35511\end{array}$

$\begin{array}{lll}-6.28232 & 1.08127 & 0.50392\end{array}$

$\begin{array}{lll}-5.54988 & 2.22512 & 0.14384\end{array}$

$\begin{array}{lll}-4.25887 & 2.088 & -0.35333\end{array}$

$\begin{array}{lll}-3.6934 & 2.97911 & -0.61663\end{array}$

$\begin{array}{lll}-5.99517 & 3.20784 & 0.26549\end{array}$

$\begin{array}{lll}-1.74415 & 1.65826 & -0.83894\end{array}$

$\begin{array}{lll}-1.87989 & -1.58325 & -1.04466\end{array}$

$\begin{array}{lll}-2.27172 & -2.52273 & -1.47279\end{array}$

$\begin{array}{lll}-1.55787 & -3.58364 & -1.19634\end{array}$

$-2.12867-4.86176-1.78205$

$\begin{array}{lll}-0.52094 & -3.58189 & -0.52562\end{array}$

$\begin{array}{lll}0.80713 & 2.89338 & 0.82616\end{array}$

$\begin{array}{lll}3.09122 & 2.61358 & -0.45897\end{array}$

$\begin{array}{llll}2.28527 & 3.59201 & 0.13231\end{array}$

$\begin{array}{lll}2.66691 & 4.93201 & 0.11326\end{array}$

$2.03921 \quad 5.6934 \quad 0.56676$

$\begin{array}{lll}4.30229 & 2.94524 & -1.06889\end{array}$

$\begin{array}{lll}4.93762 & 2.1875 & -1.51466\end{array}$

$4.68194 .28753-1.08009$

$\begin{array}{llll}5.62093 & 4.56789 & -1.54744\end{array}$

$3.87444 \quad 5.27224-0.4975$

$\begin{array}{lll}4.19033 & 6.31076 & -0.5181\end{array}$

\section{S44}




\begin{tabular}{rrr}
-2.32154 & 0.62746 & -2.63586 \\
-1.47749 & -5.7073 & -1.5549 \\
-2.24359 & -4.75743 & -2.86602 \\
-3.12706 & -5.04326 & -1.36948 \\
-6.26266 & -1.07192 & 0.63293 \\
-3.96254 & -1.29049 & -0.25908 \\
-2.15565 & -0.84543 & 2.17754 \\
-0.55695 & -1.03903 & 2.93136 \\
-1.30529 & 0.60941 & -3.04686 \\
-2.85663 & 1.48383 & -3.06317 \\
-2.83328 & -0.28912 & -2.94743 \\
3.11825 & 0.14134 & -0.97883 \\
3.95369 & -0.20412 & -0.36106 \\
3.51154 & 0.45023 & -1.95137 \\
2.5567 & -2.23308 & -1.82048 \\
\hline 1.84824 & -3.02074 & -1.53743 \\
3.96685 & -2.68438 & -1.40099 \\
4.18687 & -3.66138 & -1.8455 \\
4.07255 & -2.7836 & -0.31765 \\
4.73876 & -1.99082 & -1.75989 \\
2.48364 & -2.08732 & -3.35118 \\
1.46314 & -1.8671 & -3.68368 \\
2.80337 & -3.01355 & -3.84162 \\
3.13831 & -1.28422 & -3.71588 \\
-7.61224 & 1.20893 & 1.02213 \\
-893 & 1.31332 & 1.44203
\end{tabular}




\section{TS for acylation of alcohol $(R)-4 s$}

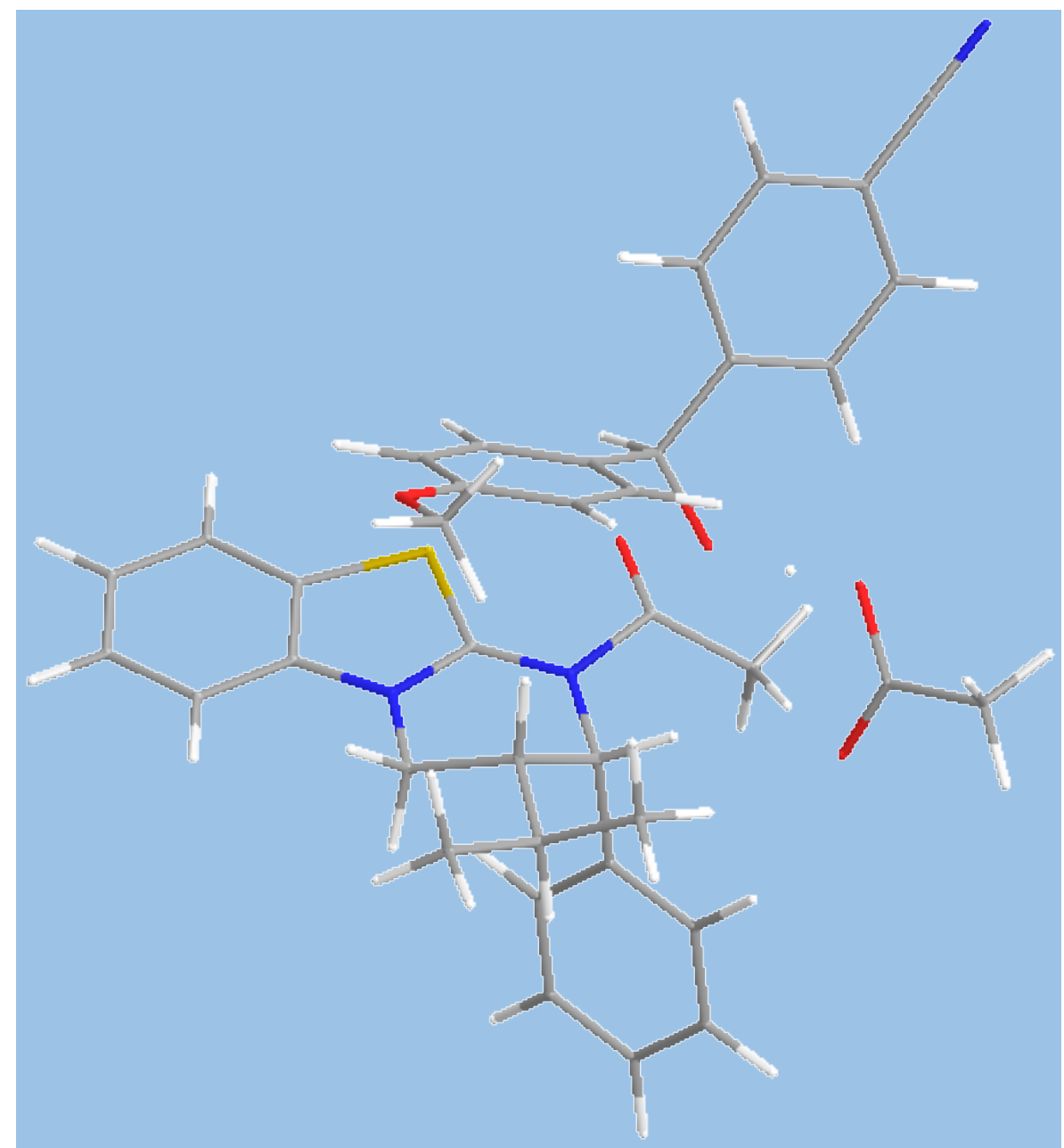

Zero-point correction=

Thermal correction to Energy=

Thermal correction to Enthalpy=

Thermal correction to Gibbs Free Energy=

Sum of electronic and zero-point Energies=

Sum of electronic and thermal Energies=

Sum of electronic and thermal Enthalpies=

Sum of electronic and thermal Free Energies=

$E(R B 3 L Y P)=-2410.79164640$

Number of imaginary frequencies: 1
0.697111 (Hartree/Particle)

0.741531

0.742476

0.615369

$-2409.399355$

$-2409.354935$

$-2409.353991$

$-2409.481098$

Charge $=0$ Multiplicity $=1$

$\begin{array}{lrll}\mathrm{C} & 0.09911 & 0.94126 & -2.12907 \\ \mathrm{C} & -0.13479 & 2.41582 & -2.31934 \\ \mathrm{H} & -0.24793 & 2.97416 & -1.39124 \\ \mathrm{O} & -0.19963 & 0.1062 & -2.97298 \\ \mathrm{~N} & 1.16612 & 0.54846 & -1.18985\end{array}$


$1.73813-0.64416-1.34665$

$\begin{array}{lll}2.67377 & -1.1212 & -0.49492\end{array}$

$\begin{array}{lll}1.91003 & 0.52182 & 1.17081\end{array}$

$\begin{array}{lll}1.54726 & 1.42768 & -0.04092\end{array}$

$\begin{array}{lll}2.61917 & 2.43565 & -0.44778\end{array}$

$\begin{array}{llll}2.48168 & 3.77001 & -0.03459\end{array}$

$\begin{array}{lll}3.4601 & 4.71437 & -0.34978\end{array}$

$\begin{array}{lll}4.58331 & 4.3484 & -1.0924\end{array}$

$\begin{array}{lll}4.7182 & 3.02984 & -1.52844\end{array}$

$\begin{array}{lll}3.74398 & 2.08267 & -1.20934\end{array}$

$\begin{array}{lll}3.86094 & 1.06955 & -1.58428\end{array}$

$\begin{array}{lll}5.5785 & 2.73605 & -2.12399\end{array}$

$\begin{array}{llll}5.34055 & 5.08665 & -1.342\end{array}$

$\begin{array}{lll}3.33348 & 5.74342 & -0.02433\end{array}$

$\begin{array}{lll}1.58931 & 4.07188 & 0.50504\end{array}$

$\begin{array}{lll}0.632 & 1.96127 & 0.21549\end{array}$

$\begin{array}{lll}1.00772 & -0.06766 & 1.37979\end{array}$

$\begin{array}{lll}-1.43347 & 0.80847 & -0.59911\end{array}$

$\begin{array}{lll}-1.98362 & -0.47038 & -0.54041\end{array}$

$\begin{array}{lll}-1.64987 & -1.17602 & 0.77867\end{array}$

$\begin{array}{lll}-0.93603 & -2.38244 & 0.7997\end{array}$

$\begin{array}{lll}-0.58925 & -3.0054 & 1.99733\end{array}$

$\begin{array}{lll}-0.95916 & -2.42749 & 3.21796\end{array}$

$\begin{array}{lll}-1.67817 & -1.22359 & 3.21924\end{array}$

$\begin{array}{lll}-2.01372 & -0.61355 & 2.00834\end{array}$

$\begin{array}{lll}-2.56182 & 0.32453 & 2.02289\end{array}$

$\begin{array}{lll}-1.98526 & -0.75927 & 4.14998\end{array}$

$\begin{array}{lll}-1.52415 & -1.07123 & -1.34937\end{array}$

$\begin{array}{lll}-2.02959 & 1.75542 & 0.12447\end{array}$

$\begin{array}{lll}-2.59586 & 2.52481 & 0.71413\end{array}$

$\begin{array}{lll}-1.88935 & 3.57923 & 1.016\end{array}$

$\begin{array}{lll}-2.65953 & 4.61921 & 1.81037\end{array}$

$\begin{array}{lll}-0.70368 & 3.75124 & 0.70539\end{array}$

$\begin{array}{lll}1.42388 & -1.74405 & -2.68648\end{array}$

$\begin{array}{lll}3.2449 & -2.34396 & -0.88276\end{array}$

$2.68414 \quad-2.82933 \quad-2.06828$

$\begin{array}{llll}3.11101 & -4.0386 & -2.61372\end{array}$

$2.67186-4.419 \quad-3.53117$

$\begin{array}{lll}4.2541 & -3.04489 & -0.21996\end{array}$

$\begin{array}{lll}4.70199 & -2.66537 & 0.69192\end{array}$

$\begin{array}{lll}4.68076 & -4.25235 & -0.77236\end{array}$

$\begin{array}{llll}5.4651 & -4.81393 & -0.27423\end{array}$

$4.11643-4.74676-1.95511$ 


$$
\begin{aligned}
& 4.46456-5.68946-2.36595 \\
& \begin{array}{lll}
-2.97975 & 4.19126 \quad 2.76681
\end{array} \\
& \begin{array}{lll}
-3.56648 & 4.9062 & 1.26828
\end{array} \\
& \begin{array}{lll}
-2.03813 & 5.4981 & 1.99009
\end{array} \\
& \begin{array}{lll}
-0.04095 & -3.94288 & 2.01008
\end{array} \\
& \begin{array}{lll}
-0.6495 & -2.84924-0.14115
\end{array} \\
& \begin{array}{lll}
-1.04246 & 2.51339 & -2.91652
\end{array} \\
& \begin{array}{lll}
0.70661 & 2.82693 & -2.89235
\end{array} \\
& 3.0144 \quad-0.45502 \quad 0.77031 \\
& \begin{array}{lll}
3.98139 & 0.05026 & 0.65759
\end{array} \\
& 3.11847-1.2428 \quad 1.51767 \\
& 2.28267 \quad 1.3033 \quad 2.46092 \\
& 3.05112 \quad 2.04243 \quad 2.1973 \\
& \begin{array}{lll}
2.8714 & 0.38092 & 3.54598
\end{array} \\
& 3.03246 \quad 0.95093 \quad 4.4673 \\
& 3.83898-0.05055 \quad 3.2654 \\
& \begin{array}{lll}
2.18457 & -0.4419 & 3.78338
\end{array} \\
& \begin{array}{lll}
1.06819 & 2.04662 \quad 3.0404
\end{array} \\
& 0.61963 \quad 2.75184 \quad 2.33744 \\
& 1.36686 \quad 2.60704 \quad 3.93404 \\
& \begin{array}{lll}
0.28999 & 1.33212 & 3.3367
\end{array} \\
& \begin{array}{lll}
-3.49209 & -0.49213-0.85007
\end{array} \\
& \begin{array}{lll}
-4.27746 & -1.62083 & -0.57034
\end{array} \\
& \begin{array}{lll}
-4.09458 & 0.59963 & -1.48589
\end{array} \\
& \begin{array}{lll}
-5.62465 & -1.66202-0.91128
\end{array} \\
& \begin{array}{lll}
-3.83047 & -2.47676 & -0.07289
\end{array} \\
& \begin{array}{lll}
-5.44243 & 0.57568 & -1.82972
\end{array} \\
& \begin{array}{lll}
-3.49272 & 1.47498 & -1.69685
\end{array} \\
& \begin{array}{lll}
-6.22051 & -0.55838 & -1.54546
\end{array} \\
& \begin{array}{lll}
-6.22324 & -2.53987 & -0.68787
\end{array} \\
& \begin{array}{lll}
-5.90079 & 1.4307 & -2.31728
\end{array} \\
& \begin{array}{lll}
-7.60966 & -0.59109 & -1.8962
\end{array} \\
& \begin{array}{llll}
-8.73796 & -0.61939 & -2.18143
\end{array} \\
& \begin{array}{lll}
-0.57434 & -3.10678 & 4.3421
\end{array} \\
& \begin{array}{lll}
-0.96967 & -2.59184 & 5.60283
\end{array} \\
& \begin{array}{lll}
-0.58274 & -3.29067 & 6.34709
\end{array} \\
& \begin{array}{lll}
-0.54394 & -1.59595 & 5.78683
\end{array} \\
& \begin{array}{lll}
-2.06253 & -2.53551 & 5.69268
\end{array}
\end{aligned}
$$




\section{TS for acylation of alcohol $(S)-4 s$}



Zero-point correction=

Thermal correction to Energy=

Thermal correction to Enthalpy=

Thermal correction to Gibbs Free Energy=

Sum of electronic and zero-point Energies=

Sum of electronic and thermal Energies=

Sum of electronic and thermal Enthalpies=

Sum of electronic and thermal Free Energies=

$E(R B 3 L Y P)=-2410.79020766$

Number of imaginary frequencies: 1

Charge $=0$ Multiplicity $=1$

$\begin{array}{llll}\mathrm{C} & 0.1401 & 0.9226 & -2.0459 \\ \mathrm{C} & -0.1044 & 2.3942 & -2.2437 \\ \mathrm{H} & -0.2039 & 2.9594 & -1.3182 \\ \mathrm{O} & -0.1751 & 0.0789 & -2.8751 \\ \mathrm{~N} & 1.2281 & 0.5412 & -1.1261 \\ \mathrm{C} & 1.7947 & -0.6545 & -1.2792 \\ & & & \mathrm{~S} 49\end{array}$

0.741530

0.742474

0.615006

$-2409.397670$

$-2409.353223$

$-2409.352279$

$-2409.479747$ 
$2.7524-1.1198 \quad-0.4454$

$2.0408 \quad 0.5524 \quad 1.2131$

$\begin{array}{lll}1.639 & 1.4382 & -0.0014\end{array}$

$\begin{array}{lll}2.6956 & 2.4428 & -0.454\end{array}$

$2.5672 \quad 3.7823 \quad-0.0549$

$3.5335 \quad 4.7241 \quad-0.4119$

$\begin{array}{lll}4.635 & 4.3501 & -1.1827\end{array}$

$\begin{array}{lll}4.7599 & 3.0261 & -1.6048\end{array}$

$\begin{array}{lll}3.7978 & 2.0815 & -1.2441\end{array}$

$3.9056 \quad 1.0636 \quad-1.6089$

$\begin{array}{lll}5.6028 & 2.7259 & -2.2217\end{array}$

$5.3828 \quad 5.0863-1.4645$

$3.4144 \quad 5.7572 \quad-0.0966$

$\begin{array}{lll}1.6905 & 4.0895 & 0.5071\end{array}$

$\begin{array}{lll}0.7304 & 1.9743 & 0.2734\end{array}$

$\begin{array}{lll}1.1461 & -0.035 & 1.4591\end{array}$

$\begin{array}{lll}-1.3625 & 0.7936 & -0.4753\end{array}$

$\begin{array}{lll}-1.9847 & -0.4551 & -0.4757\end{array}$

$\begin{array}{lll}-1.6145 & -1.2379 & 0.7891\end{array}$

$\begin{array}{lll}-1.0087 & -2.4983 & 0.7102\end{array}$

$\begin{array}{lll}-0.6165 & -3.1881 & 1.8567\end{array}$

$\begin{array}{lll}-0.8302 & -2.6152 \quad 3.1197\end{array}$

$\begin{array}{lll}-1.4496 & -1.353 & 3.2129\end{array}$

$\begin{array}{lll}-1.8329 & -0.6785 & 2.0602\end{array}$

$\begin{array}{lll}-2.3005 & 0.2989 & 2.1347\end{array}$

$\begin{array}{lll}-1.6246 & -0.916 & 4.1915\end{array}$

$\begin{array}{lll}-1.583 & -1.0275 & -1.3334\end{array}$

$\begin{array}{lll}-1.9394 & 1.7443 & 0.2616\end{array}$

$\begin{array}{lll}-2.4715 & 2.5049 & 0.8925\end{array}$

$\begin{array}{lll}-1.7594 & 3.566 & 1.1521\end{array}$

$\begin{array}{lll}-2.4919 & 4.5989 & 1.9901\end{array}$

$\begin{array}{lll}-0.5948 & 3.7494 & 0.7738\end{array}$

$\begin{array}{lll}1.441 & -1.7762 & -2.5907\end{array}$

$3.3084-2.352 \quad-0.8265$

$2.7133-2.856 \quad-1.9872$

$3.1202-4.0768-2.5221$

$2.6549-4.4715-3.4205$

$\begin{array}{lll}4.3324 & -3.0455 & -0.1787\end{array}$

$\begin{array}{lll}4.8067 & -2.6524 & 0.7138\end{array}$

$4.7383-4.2649-0.7203$

$\begin{array}{llll}5.533 & -4.8214 & -0.233\end{array}$

$\begin{array}{lll}4.14 & -4.7779 & -1.8782\end{array}$

$\begin{array}{lll}4.4727 & -5.7296 & -2.2809\end{array}$ 


\begin{tabular}{|c|c|c|}
\hline-2.749 & 4.1692 & 2.9646 \\
\hline-3.4329 & 4.8742 & 1.503 \\
\hline-1.8706 & 5.4849 & 2.1312 \\
\hline-0.1461 & -4.164 & 1.7821 \\
\hline-0.8413 & -2.9474 & -0.2662 \\
\hline-1.0236 & 2.4819 & -2.8247 \\
\hline 0.7241 & 2.8049 & -2.8355 \\
\hline 3.1346 & -0.4285 & 0.7941 \\
\hline 4.0945 & 0.0787 & 0.6385 \\
\hline 3.2694 & -1.2006 & 1.5527 \\
\hline 2.4503 & 1.3545 & 2.4796 \\
\hline 3.2033 & 2.0952 & 2.1789 \\
\hline 3.0829 & 0.4541 & 3.5583 \\
\hline 3.272 & 1.0406 & 4.4636 \\
\hline 4.0432 & 0.0246 & 3.2512 \\
\hline 2.4121 & -0.369 & 3.8367 \\
\hline 1.2503 & 2.0985 & 3.0874 \\
\hline 0.7738 & 2.7896 & 2.3888 \\
\hline 1.5736 & 2.674 & 3.9624 \\
\hline 0.4878 & 1.3839 & 3.4225 \\
\hline-3.5066 & -0.3814 & -0.693 \\
\hline-4.3633 & -1.4213 & -0.3244 \\
\hline-4.0652 & 0.7226 & -1.3528 \\
\hline-5.7353 & -1.3752 & -0.5937 \\
\hline-3.9677 & -2.2939 & 0.1888 \\
\hline-5.4251 & 0.7866 & -1.6277 \\
\hline-3.4173 & 1.5431 & -1.6392 \\
\hline-6.2725 & -0.2637 & -1.2497 \\
\hline-6.3647 & -2.2023 & -0.2843 \\
\hline-5.8575 & 1.6452 & -2.133 \\
\hline-7.5967 & -0.1055 & -1.5627 \\
\hline-8.4945 & -1.1368 & -1.1955 \\
\hline-9.4813 & -0.8117 & -1.5321 \\
\hline-8.2423 & -2.0886 & -1.6833 \\
\hline-8.5161 & -1.2889 & -0.1076 \\
\hline-0.4106 & -3.3057 & 4.3034 \\
\hline .0 & -3.8637 & 5 . \\
\hline
\end{tabular}




\section{TS for acylation of alcohol $(R)-4 u$}



Zero-point correction=

Thermal correction to Energy=

Thermal correction to Enthalpy=

Thermal correction to Gibbs Free Energy=

Sum of electronic and zero-point Energies=

Sum of electronic and thermal Energies=

Sum of electronic and thermal Enthalpies=

Sum of electronic and thermal Free Energies=

$E(R B 3 L Y P)=-2318.51538418$

Number of imaginary frequencies: 1

$\begin{array}{lrrr}\text { Charge }=\text { 0 Multiplicity }=1 & & \\ \text { C } & 0.01598 & 0.98722 & -2.16455 \\ \mathrm{C} & -0.29866 & 2.44865 & -2.34828 \\ \mathrm{H} & -0.57891 & 2.96445 & -1.43088 \\ \mathrm{O} & -0.06727 & 0.17203 & -3.07592 \\ \mathrm{~N} & 0.97971 & 0.64467 & -1.09303 \\ \mathrm{C} & 1.67253 & -0.48481 & -1.2064 \\ \mathrm{~N} & 2.51485 & -0.924 & -0.24276 \\ \mathrm{C} & 1.37482 & 0.56882 & 1.34884\end{array}$




$$
\begin{aligned}
& \begin{array}{lll}
1.11483 & 1.49729 & 0.12767
\end{array} \\
& 2.14566 \quad 2.60417 \quad-0.08185 \\
& \begin{array}{lll}
1.83736 & 3.90324 & 0.35146
\end{array} \\
& \begin{array}{lll}
2.76774 & 4.93517 & 0.21719
\end{array} \\
& \begin{array}{lll}
4.01377 \quad 4.69356 & -0.36251
\end{array} \\
& \begin{array}{lll}
4.32145 & 3.41119 & -0.81831
\end{array} \\
& \begin{array}{llll}
3.39488 & 2.37659 & -0.67971
\end{array} \\
& 3.65037 \quad 1.39419-1.06718 \\
& \begin{array}{llll}
5.28082 & 3.21365 & -1.28929
\end{array} \\
& \begin{array}{lll}
4.73406 & 5.49987 & -0.47167
\end{array} \\
& 2.50849 \quad 5.93502 \quad 0.55501 \\
& \begin{array}{lll}
0.85343 & 4.10826 & 0.76193
\end{array} \\
& \begin{array}{lll}
0.13113 & 1.94722 & 0.26396
\end{array} \\
& 0.50587-0.09993 \quad 1.40174 \\
& \begin{array}{lll}
-1.66369 & 0.66503 & -0.91586
\end{array} \\
& \begin{array}{lll}
-2.11903-0.65826 & -0.97879
\end{array} \\
& \begin{array}{lll}
-1.91433 & -1.37919 & 0.35683
\end{array} \\
& \begin{array}{lll}
-1.09887 & -2.5151 & 0.45548
\end{array} \\
& \begin{array}{lll}
-0.86917 & -3.14674 & 1.67685
\end{array} \\
& \begin{array}{lll}
-1.46375 & -2.64928 \quad 2.84256
\end{array} \\
& \begin{array}{lll}
-2.28893 & -1.51828 \quad 2.76543
\end{array} \\
& \begin{array}{lll}
-2.50466 & -0.8987 & 1.53222
\end{array} \\
& \begin{array}{lll}
-3.13828 & -0.01731 & 1.48556
\end{array} \\
& \begin{array}{lll}
-2.77018 & -1.11836 & 3.65133
\end{array} \\
& \begin{array}{lll}
-1.49348 & -1.18129-1.72566
\end{array} \\
& \begin{array}{lll}
-2.42741 & 1.51333 & -0.29384
\end{array} \\
& \begin{array}{lll}
-3.15065 & 2.2231 & 0.23691
\end{array} \\
& \begin{array}{lll}
-2.59459 & 3.30287 & 0.70224
\end{array} \\
& \begin{array}{lll}
-3.56582 & 4.23484 & 1.40835
\end{array} \\
& \begin{array}{lll}
-1.39133 & 3.58529 & 0.60536
\end{array} \\
& \begin{array}{lll}
1.65511 & -1.54048 & -2.61787
\end{array} \\
& 3.24351-2.07375-0.58604 \\
& 2.90583-2.54543-1.85854 \\
& 3.51435-3.68653-2.37746 \\
& 3.24816-4.05706-3.36298 \\
& 4.20511-2.71955 \quad 0.19316 \\
& \begin{array}{lll}
4.47989 & -2.34909 & 1.17474
\end{array} \\
& \begin{array}{lll}
4.81437 & -3.85868 & -0.33301
\end{array} \\
& \begin{array}{llll}
5.56562 & -4.37641 & 0.25554
\end{array} \\
& 4.47385-4.33987-1.60336 \\
& \begin{array}{lll}
4.96099 & -5.22891 & -1.99205
\end{array} \\
& \begin{array}{lll}
-3.98844 & 3.73162 & 2.28522
\end{array} \\
& \begin{array}{lll}
-4.40172 & 4.47591 & 0.74362
\end{array}
\end{aligned}
$$




\begin{tabular}{|c|c|c|}
\hline 3 . & 23 & 1 \\
\hline-0.24139 & -4.03025 & 171851 \\
\hline 3803 & -2 & 0 \\
\hline 1 . & 2 . & -3 \\
\hline 0 . & 2 . & 2 \\
\hline 2 . & -0 . & \\
\hline 3. & & \\
\hline 2 . & -1 & \\
\hline 1. & 1. & \\
\hline 2 . & & \\
\hline 1 . & & \\
\hline 1. & & \\
\hline 3. & & \\
\hline 1.35106 & -0 & 3 \\
\hline 0 & 1.9335 & \\
\hline 0.25 & 2.630 & 2 \\
\hline 0.26308 & 2 . & $A$ \\
\hline 0.60313 & 1 . & 3. \\
\hline-3.56144 & -0.77591 & -1.5034 \\
\hline-4.29965 & -1.95644 & -1 \\
\hline-4.13762 & 0.274 & -2 \\
\hline-5.5798 & -2.08225 & $-1 \cdot 8$ \\
\hline-3.87234 & -2.78397 & -0.77 \\
\hline-5.41971 & 0.15299 & -2.7 \\
\hline-3.57507 & 1.19223 & -2.35 \\
\hline-6.14708 & -1.025 & -2.5 \\
\hline-6.13593 & -3.00 & $-1 \cdot 7$ \\
\hline-5.85148 & 0.98454 & $-3 \cdot 3$ \\
\hline-1.17948 & -3.33 & - 9 \\
\hline-1.799 & -2.85 & 5.1 \\
\hline-1.457 & $-3.5 \varepsilon$ & 5.9 \\
\hline-1.50296 & -1.87329 & 5.4 \\
\hline 89392 & -2 & 5.127 \\
\hline 19 & 2015 & \\
\hline
\end{tabular}




\section{TS for acylation of alcohol $(S)-4 u$}

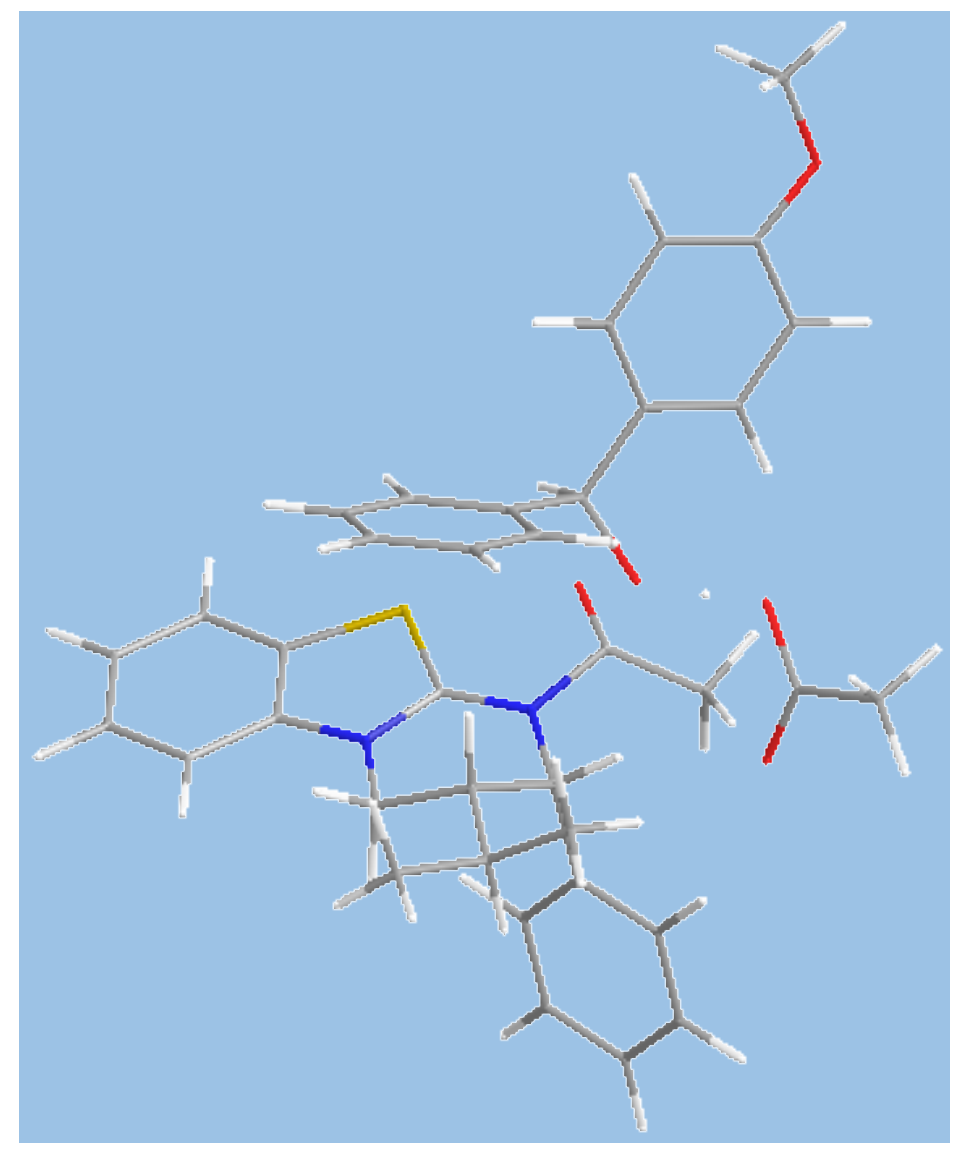

Zero-point correction=

Thermal correction to Energy=

Thermal correction to Enthalpy=

Thermal correction to Gibbs Free Energy=

Sum of electronic and zero-point Energies=

Sum of electronic and thermal Energies=

Sum of electronic and thermal Enthalpies=

Sum of electronic and thermal Free Energies=
0.698127 (Hartree/Particle)

0.740717

0.741661

0.619189

$-2317.150126$

$-2317.107536$

$-2317.106592$

$-2317.229064$

$E(R B 3 L Y P)=-2318.51509673$

Number of imaginary frequencies: 1

$\begin{array}{lccc}\text { Charge }=0 \text { Multiplicity }=1 & & \\ \text { C } & 0.1048 & 0.36446 & -2.01132 \\ \mathrm{C} & -0.09954 & 1.75752 & -2.5452 \\ \mathrm{H} & -0.1726 & 2.52515 & -1.776 \\ \mathrm{O} & -0.21576 & -0.63896 & -2.63728 \\ \mathrm{~N} & 1.19861 & 0.1807 & -1.03187 \\ \mathrm{C} & 1.74632 & -1.02589 & -0.91837 \\ \mathrm{~N} & 2.6947 & -1.31276 & 0.00309 \\ \mathrm{C} & 1.99659 & 0.68918 & 1.25472\end{array}$




$$
\begin{aligned}
& \begin{array}{lll}
1.62361 & 1.29461 & -0.12895
\end{array} \\
& 2.70593 \quad 2.1523 \quad-0.78034 \\
& 2.60254 \quad 3.5494 \quad-0.69229 \\
& \begin{array}{llll}
3.59191 & 4.36944 & -1.23744
\end{array} \\
& \begin{array}{lll}
4.69217 & 3.81254 & -1.89035
\end{array} \\
& \begin{array}{lll}
4.7926 & 2.42554 & -2.00462
\end{array} \\
& 3.80747 \quad 1.60355-1.45513 \\
& \begin{array}{llll}
3.8971 & 0.52816 & -1.582
\end{array} \\
& \begin{array}{llll}
5.63436 & 1.97934 & -2.52768
\end{array} \\
& \begin{array}{llll}
5.45797 & 4.45314 & -2.31938
\end{array} \\
& 3.4916 \quad 5.44895-1.16255 \\
& \begin{array}{llll}
1.72692 & 3.99034 & -0.22589
\end{array} \\
& \begin{array}{lll}
0.72632 & 1.89796 & 0.01086
\end{array} \\
& \begin{array}{lll}
1.08794 & 0.18761 & 1.61211
\end{array} \\
& \begin{array}{lll}
-1.36114 & 0.61313 & -0.48449
\end{array} \\
& \begin{array}{lll}
-1.95999 & -0.60288 & -0.13079
\end{array} \\
& \begin{array}{lll}
-1.57503-1.01 & 1.29553
\end{array} \\
& \begin{array}{lll}
-0.92324 & -2.22234 & 1.54982
\end{array} \\
& \begin{array}{lll}
-0.53264 & -2.57607 & 2.84648
\end{array} \\
& \begin{array}{lll}
-0.79325 & -1.7146 \quad 3.91165
\end{array} \\
& \begin{array}{lll}
-1.4504 & -0.50215 \quad 3.6709
\end{array} \\
& \begin{array}{lll}
-1.83676 & -0.15329 & 2.37728
\end{array} \\
& \begin{array}{lll}
-2.34055 & 0.79154 & 2.19327
\end{array} \\
& \begin{array}{lll}
-1.6673 & 0.17002 & 4.49793
\end{array} \\
& \begin{array}{lll}
-1.54901 & -1.3749 & -0.80733
\end{array} \\
& \begin{array}{lll}
-1.92403 & 1.69149 & -0.0279
\end{array} \\
& \begin{array}{lll}
-2.46881 & 2.60994 & 0.38281
\end{array} \\
& \begin{array}{lll}
-1.73885 & 3.68478 & 0.43545
\end{array} \\
& \begin{array}{lll}
-2.4731 & 4.89594 & 0.98695
\end{array} \\
& \begin{array}{lll}
-0.55198 & 3.76045 & 0.08404
\end{array} \\
& 1.38159-2.40018-1.95978 \\
& 3.23648-2.60338-0.10157 \\
& 2.63949-3.33856-1.13065 \\
& 3.03454-4.64981-1.38808 \\
& 2.56737-5.22339-2.18317 \\
& \begin{array}{lll}
4.25054 & -3.15238 & 0.68536
\end{array} \\
& \begin{array}{lll}
4.72645 & -2.58053 & 1.47452
\end{array} \\
& \begin{array}{lll}
4.64526 & -4.46375 & 0.42035
\end{array} \\
& \begin{array}{lll}
5.43315 & -4.91029 & 1.01926
\end{array} \\
& 4.04475 \quad-5.2071 \quad-0.60343 \\
& \begin{array}{lll}
4.36827 & -6.22633-0.7913
\end{array} \\
& \begin{array}{lll}
-2.76842 \quad 4.70652 & 2.02507
\end{array} \\
& \begin{array}{lll}
-3.39302 & 5.06557 & 0.41812
\end{array}
\end{aligned}
$$




\begin{tabular}{|c|c|c|}
\hline-1.83666 & 5.78155 & 0.94255 \\
\hline-0.0309 & -3.52523 & 3.02102 \\
\hline-0.72419 & -2.90187 & 0.72292 \\
\hline-1.02206 & 1.73636 & -3.12724 \\
\hline 0.73383 & 1.99314 & -3.21994 \\
\hline 3.07279 & -0.38049 & 1.07449 \\
\hline 4.04718 & 0.0623 & 0.83404 \\
\hline 3.17634 & -0.97639 & 1.98241 \\
\hline 2.41486 & 1.73971 & 2.32006 \\
\hline 3.20013 & 2.37045 & 1.88172 \\
\hline 2.99416 & 1.07716 & 3.58521 \\
\hline 3.1863 & 1.84027 & 4.34708 \\
\hline 3.94402 & 0.56091 & 3.4046 \\
\hline 2.28588 & 0.3557 & 4.01287 \\
\hline 1.23414 & 2.63799 & 2.72306 \\
\hline 0.78941 & 3.17042 & 1.87961 \\
\hline 1.56751 & 3.38369 & 3.45433 \\
\hline 0.44332 & 2.03926 & 3.19166 \\
\hline-3.48107 & -0.61157 & -0.35983 \\
\hline-4.32197 & -1.52388 & 0.28196 \\
\hline-4.05643 & 0.259 & -1.29669 \\
\hline-5.69363 & -1.5768 & 0.01213 \\
\hline-3.91147 & -2.21271 & 1.01539 \\
\hline-5.41667 & 0.22269 & -1.57603 \\
\hline-3.42184 & 0.98032 & -1.7986 \\
\hline-6.24718 & -0.69754 & -0.92304 \\
\hline-6.31025 & -2.29761 & 0.53788 \\
\hline-5.86172 & 0.90271 & -2.2965 \\
\hline-7.57421 & -0.65406 & -1.26548 \\
\hline-8.45406 & -1.56035 & -0.6276 \\
\hline-9.44581 & -1.35835 & -1.0382 \\
\hline-8.18378 & -2.60513 & -0.8352 \\
\hline-8.4763 & -1.40876 & 0.46054 \\
\hline-0.49757 & -1.98595 & 4.92212 \\
\hline
\end{tabular}




\section{References}

(1) Greenhalgh, M. D.; Taylor, J. E.; Smith, A. D. Best Practice Considerations for Using the Selectivity Factor, s, as a Metric for the Efficiency of Kinetic Resolutions. Tetrahedron 2018, 74 (38), 55545560. https://doi.org/10.1016/j.tet.2018.05.069.

(2) Shaw, S. A.; Aleman, P.; Christy, J.; Kampf, J. W.; Va, P.; Vedejs, E. Enantioselective TADMAPCatalyzed Carboxyl Migration Reactions for the Synthesis of Stereogenic Quaternary Carbon. J. Am. Chem. Soc. 2006, 128 (3), 925-934. https://doi.org/10.1021/ja056150x

(3) Kinens, A.; Sejejs, M.; Kamlet, A. S.; Piotrowski, D. W.; Vedejs, E.; Suna, E. Development of a Chiral DMAP Catalyst for the Dynamic Kinetic Resolution of Azole Hemiaminals. J. Org. Chem. 2017, 82 (2), 869-886. https://doi.org/10.1021/acs.joc.6b02955.

(4) Duffey, T. A.; Shaw, S. A.; Vedejs, E. AcOLeDMAP and BnOLeDMAP: Conformationally Restricted Nucleophilic Catalysts for Enantioselective Rearrangement of Indolyl Acetates and Carbonates. J. Am. Chem. Soc. 2009, 131 (1), 14-15. https://doi.org/10.1021/ja805541u.

(5) Ishihara, K.; Kosugi, Y.; Akakura, M. Rational Design of an L -Histidine-Derived Minimal Artificial Acylase for the Kinetic Resolution of Racemic Alcohols. J. Am. Chem. Soc. 2004, 126 (39), 1221212213. https://doi.org/10.1021/ja045850j.

(6) Mandai, H.; Irie, S.; Mitsudo, K.; Suga, S. Studies on the Synthesis of DMAP Derivatives by Diastereoselective Ugi Reactions. Molecules 2011, 16 (10), 8815-8832. https://doi.org/10.3390/molecules16108815.

(7) Fu, G. C. Asymmetric Catalysis with "Planar-Chiral" Derivatives of 4-(Dimethylamino)Pyridine. Acc. Chem. Res. 2004, 37 (8), 542-547. https://doi.org/10.1021/ar030051b.

(8) Gleeson, O.; Gun'ko, Y.; Connon, S. (S)-Proline-Derived Catalysts for the Acylative Kinetic Resolution of Alcohols: A Remote Structural Change Allows a Complete Selectivity Switch. Synlett 2013, 24 (13), 1728-1734. https://doi.org/10.1055/s-0033-1339286.

(9) Li, X.; Jiang, H.; Uffman, E. W.; Guo, L.; Zhang, Y.; Yang, X.; Birman, V. B. Kinetic Resolution of Secondary Alcohols Using Amidine-Based Catalysts. J. Org. Chem. 2012, 77 (4), 1722-1737. https://doi.org/10.1021/jo202220x.

(10) Birman, V. B.; Li, X. Benzotetramisole: A Remarkably Enantioselective Acyl Transfer Catalyst. Org. Lett. 2006, 8 (7), 1351-1354. https://doi.org/10.1021/o1060065s.

(11) Wolfson, A.; Yehuda, C.; Shokin, O.; Tavor, D. Simple and Recyclable Oxidation, Racemization and Dynamic Kinetic Resolution of Activated Alcohols Catalyzed by Hydrated Ruthenium Chloride in Aqueous Medium. Lett. Org. Chem. 2006, 3 (2), 107-110.

https://doi.org/10.2174/157017806775224260.

(12) Cao, H.; Cai, L.-H.; Wang, C.-X.; Zhu, X.-H.; Li, Z.-M.; Hou, X.-F. Ligand Effect in Racemization and Dynamic Kinetic Resolution of Alcohols: Mechanism on Cymene Ruthenium Complexes. J. Organomet. Chem. 2015, 775, 60-66. https://doi.org/10.1016/j.jorganchem.2014.10.011.

(13) Menashe, N.; Salant, E.; Shvo, Y. Efficient Catalytic Reduction of Ketones with Formic Acid and Ruthenium Complexes. J. Organomet. Chem. 1996, 514 (1-2), 97-102. https://doi.org/10.1016/0022328X(95)05995-2.

(14) Kim, N.; Ko, S.-B.; Kwon, M. S.; Kim, M.-J.; Park, J. Air-Stable Racemization Catalyst for Dynamic Kinetic Resolution of Secondary Alcohols at Room Temperature. Org. Lett. 2005, 7 (20), 4523-4526. https://doi.org/10.1021/o1051889x.

(15) Marozsán, N.; Horváth, H.; Kováts, É.; Udvardy, A.; Erdei, A.; Purgel, M.; Joó, F. Catalytic Racemization of Secondary Alcohols with New (Arene)Ru(II)-NHC and (Arene)Ru(II)-NHC-Tertiary Phosphine Complexes. Mol. Catal. 2018, 445, 248-256. https://doi.org/10.1016/j.mcat.2017.11.040.

(16) Dijksman, A.; Elzinga, J. M.; Li, Y.-X.; Arends, I. W. C. E.; Sheldon, R. A. Efficient RutheniumCatalyzed Racemization of Secondary Alcohols: Application to Dynamic Kinetic Resolution.

Tetrahedron Asymmetry 2002, 13 (8), 879-884. https://doi.org/10.1016/S0957-4166(02)00187-8. 
(17) Wu, W.; You, C.; Yin, C.; Liu, Y.; Dong, X.-Q.; Zhang, X. Enantioselective and Diastereoselective Construction of Chiral Amino Alcohols by Iridium-f-Amphox-Catalyzed Asymmetric Hydrogenation via Dynamic Kinetic Resolution. Org. Lett. 2017, 19 (10), 2548-2551. https://doi.org/10.1021/acs.orglett.7b00844.

(18) Arita, S.; Koike, T.; Kayaki, Y.; Ikariya, T. Synthesis and Reactivities of Cp*Ir Amide and Hydride Complexes Bearing C-N Chelate Ligands. Organometallics 2008, 27 (12), 2795-2802. https://doi.org/10.1021/om800124f.

(19) Moulin, S.; Dentel, H.; Pagnoux-Ozherelyeva, A.; Gaillard, S.; Poater, A.; Cavallo, L.; Lohier, J.-F.; Renaud, J.-L. Bifunctional (Cyclopentadienone)Iron-Tricarbonyl Complexes: Synthesis, Computational Studies and Application in Reductive Amination. Chem. - Eur. J. 2013, 19 (52), 17881-17890. https://doi.org/10.1002/chem.201302432.

(20) Martín-Matute, B.; Edin, M.; Bogár, K.; Kaynak, F. B.; Bäckvall, J.-E. Combined Ruthenium(II) and Lipase Catalysis for Efficient Dynamic Kinetic Resolution of Secondary Alcohols. Insight into the Racemization Mechanism. J. Am. Chem. Soc. 2005, 127 (24), 8817-8825. https://doi.org/10.1021/ja051576x.

(21) Csjernyik, G.; Bogár, K.; Bäckvall, J.-E. New Efficient Ruthenium Catalysts for Racemization of Alcohols at Room Temperature. Tetrahedron Lett. 2004, 45 (36), 6799-6802. https://doi.org/10.1016/j.tetlet.2004.07.013.

(22) Manzini, S.; Blanco, C. A. U.; Nolan, S. P. Ruthenium Phenylindenyl Complex as an Efficient Transfer Hydrogenation Catalyst. Adv. Synth. Catal. 2012, 354 (16), 3036-3044. https://doi.org/10.1002/adsc.201200411.

(23) Frisch, M. J.; Trucks, G. W.; Schlegel, H. B.; Scuseria, G. E.; Robb, M. A.; Cheeseman, J. R.; Scalmani, G.; Barone, V.; Mennucci, B.; Petersson, G. A.; Nakatsuji, H.; Caricato, M.; Li, X.; Hratchian, H. P.; Izmaylov, A. F.; Bloino, J.; Zheng, G.; Sonnenberg, J. L.; Hada, M.; Ehara, M.; Toyota, K.; Fukuda, R.; Hasegawa, J.; Ishida, M.; Nakajima, T.; Honda, Y.; Kitao, O.; Nakai, H.; Vreven, T.; Jr., J. A. M.; Peralta, J. E.; Ogliaro, F.; Bearpark, M.; Heyd, J. J.; Brothers, E.; Kudin, K. N.; Staroverov, V. N.; Keith, T.; Kobayashi, R.; Normand, J.; Raghavachari, K.; Rendell, A.; Burant, J. C.; Iyengar, S. S.; Tomasi, J.; Cossi, M.; Rega, N.; Millam, J. M.; Klene, M.; Knox, J. E.; Cross, J. B.; Bakken, V.; Adamo, C.; Jaramillo, J.; Gomperts, R.; Stratmann, R. E.; Yazyev, O.; Austin, A. J.; Cammi, R.; Pomelli, C.; Ochterski, J. W.; Martin, R. L.; Morokuma, K.; Zakrzewski, V. G.; Voth, G. A.; Salvador, P.; Dannenberg, J. J.; Dapprich, S.; Daniels, A. D.; Farkas, O.; Foresman, J. B.; Ortiz, J. V.; Cioslowski, J.; Fox, D. J. Gaussian 09, Revision D.01; Gaussian, Inc., Wallingford CT, 2013.

(24) Bootsma, A. N.; Wheeler, S. Popular Integration Grids Can Result in Large Errors in DFTComputed Free Energies; preprint; 2019. https://doi.org/10.26434/chemrxiv.8864204.v4. 


\section{HRMS-APCI data for esters 5}

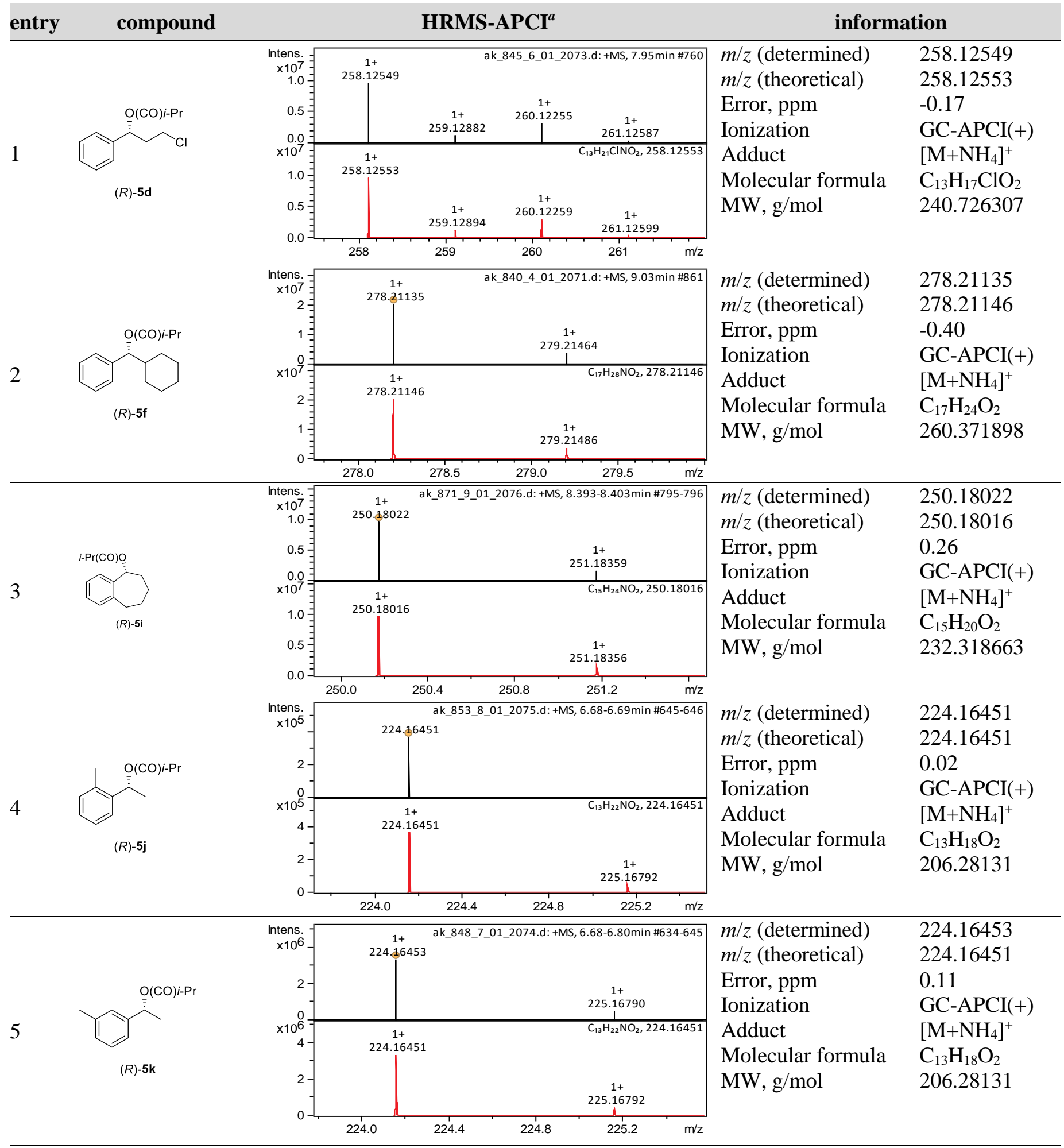




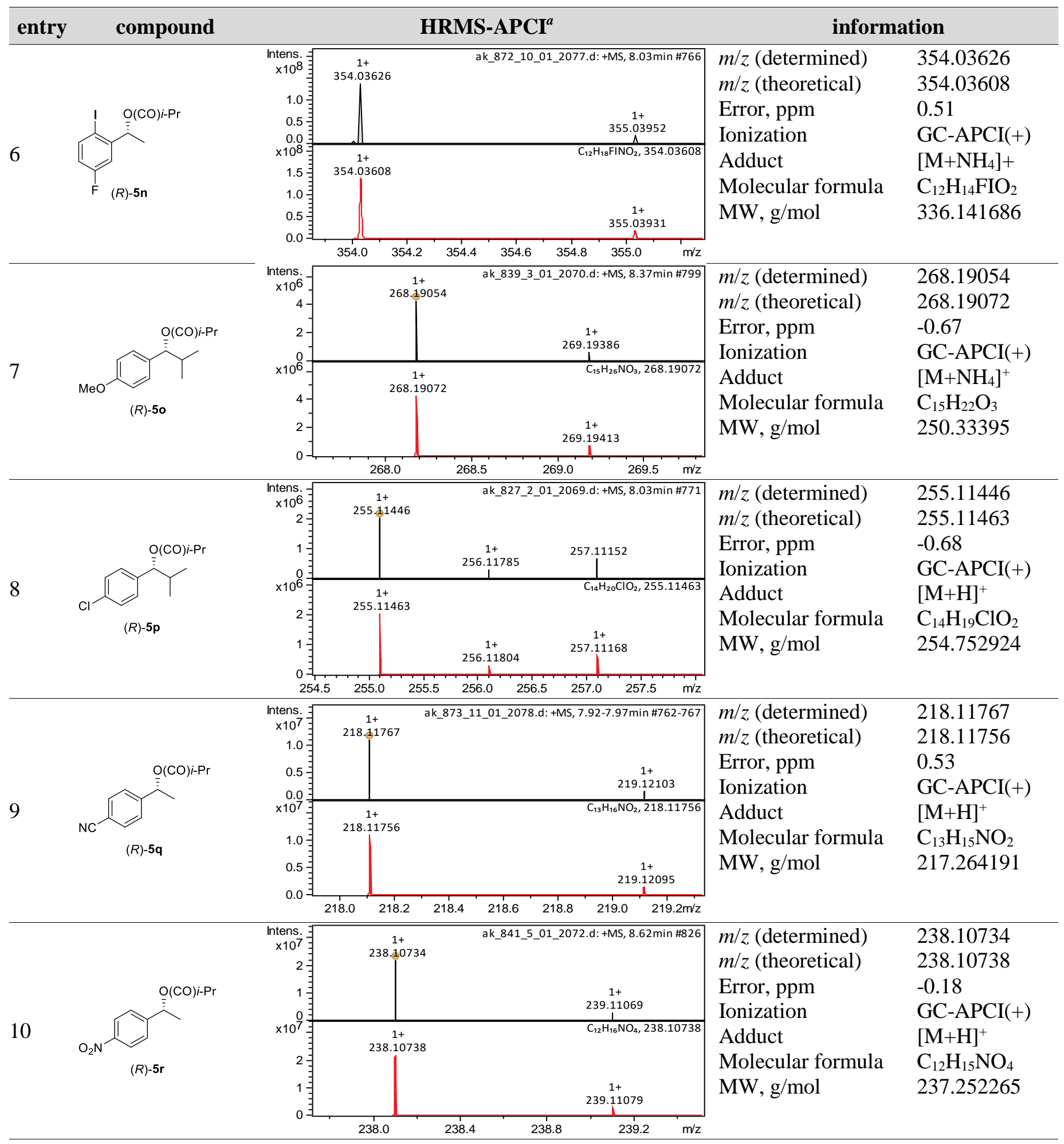




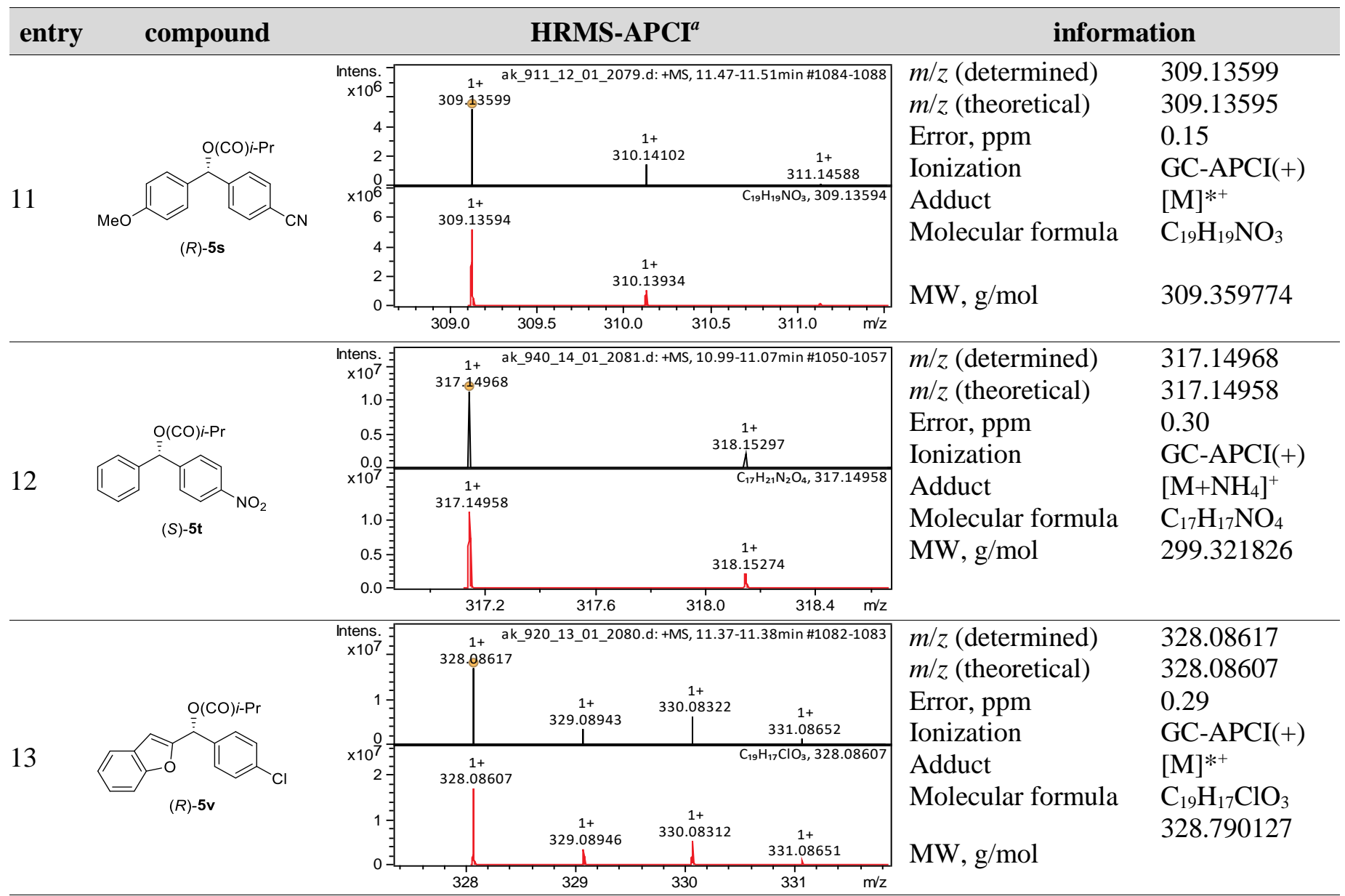

${ }^{a}$ Upper trace: measured mass spectrum, lower trace: theoretically calculated mass spectrum. 


\section{Chiral stationary phase GC data for 5a}



Area Percent Report


$* * *$ End of Report ***

*** End of Report *** 


\section{Chiral stationary phase HPLC data for $5 b-v(U V$ detection at $210 \mathrm{~nm}$ )}

Name: ak_782

Column: Chiralpak IC $4.6 \mathrm{~mm} \times 250 \mathrm{~mm}, 5 \mathrm{um}$

Flow: $1.0 \mathrm{~mL} / \mathrm{min}$

. $15 \%$ Meptane

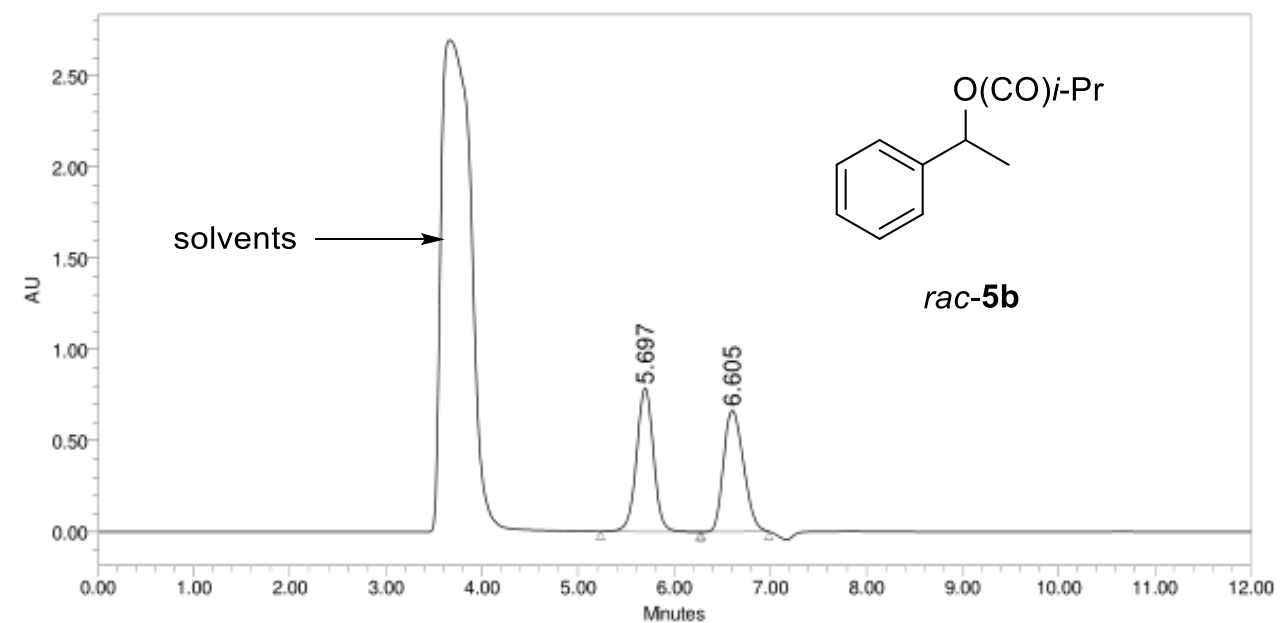

\begin{tabular}{|c|c|c|c|r|}
\hline & RT & Height & Area & \% Area \\
\hline 1 & 5.697 & 789676 & 9728927 & 50.22 \\
\hline 2 & 6.605 & 666748 & 9642814 & 49.78 \\
\hline
\end{tabular}
Name: ak_785

Column: Chiralpak IC $4.6 \mathrm{~mm} \times 250 \mathrm{~mm}, 5 \mathrm{um}$

Flow: $1.0 \mathrm{~mL} / \mathrm{mi}$

Eluent: $15 \%$ MTBE, $85 \%$ Heptane

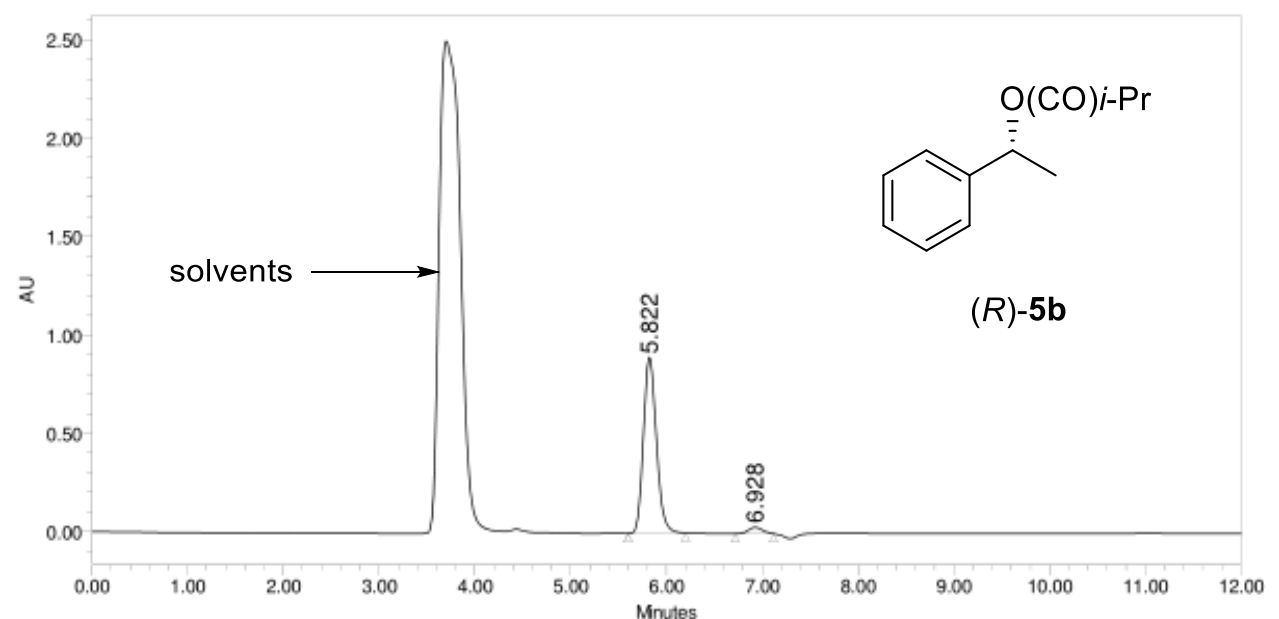

\begin{tabular}{|c|c|r|r|r|}
\hline & RT & Height & Area & \% Area \\
\hline 1 & 5.822 & 898379 & 8451900 & 96.23 \\
\hline 2 & 6.928 & 32540 & 331336 & 3.77 \\
\hline
\end{tabular} 
Name: ak_836

Column: Chiralpak IC $4.6 \mathrm{~mm}$ x $250 \mathrm{~mm}, 5$ um

.

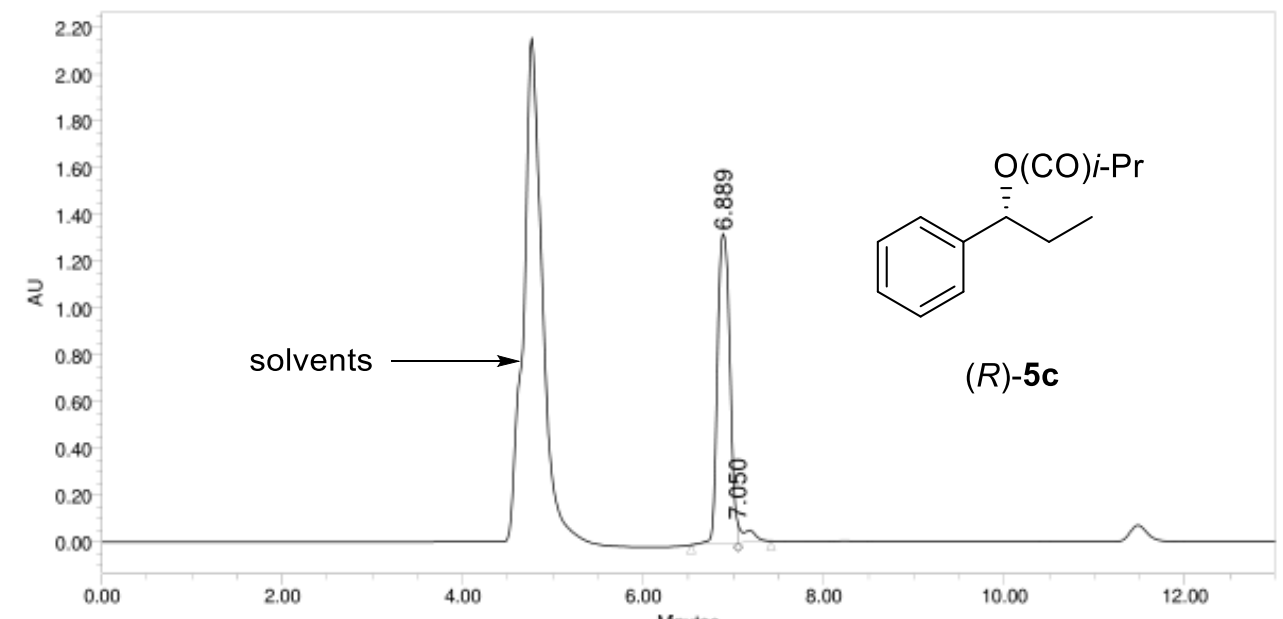

\begin{tabular}{|l|c|r|r|r|}
\hline & RT & Height & \multicolumn{1}{c|}{ Area } & \% Area \\
\hline 1 & 6.889 & 1331572 & 12589392 & 95.19 \\
\hline 2 & 7.050 & 80946 & 635470 & 4.81 \\
\hline
\end{tabular}
Name: ak_825

Column: Chiralpak IC $4.6 \mathrm{~mm} \times 250 \mathrm{~mm}, 5 \mathrm{um}$

Flow: $1.0 \mathrm{~mL} / \mathrm{min}$

Fluent: $2 \%$ IPA, $98 \%$ Heptane

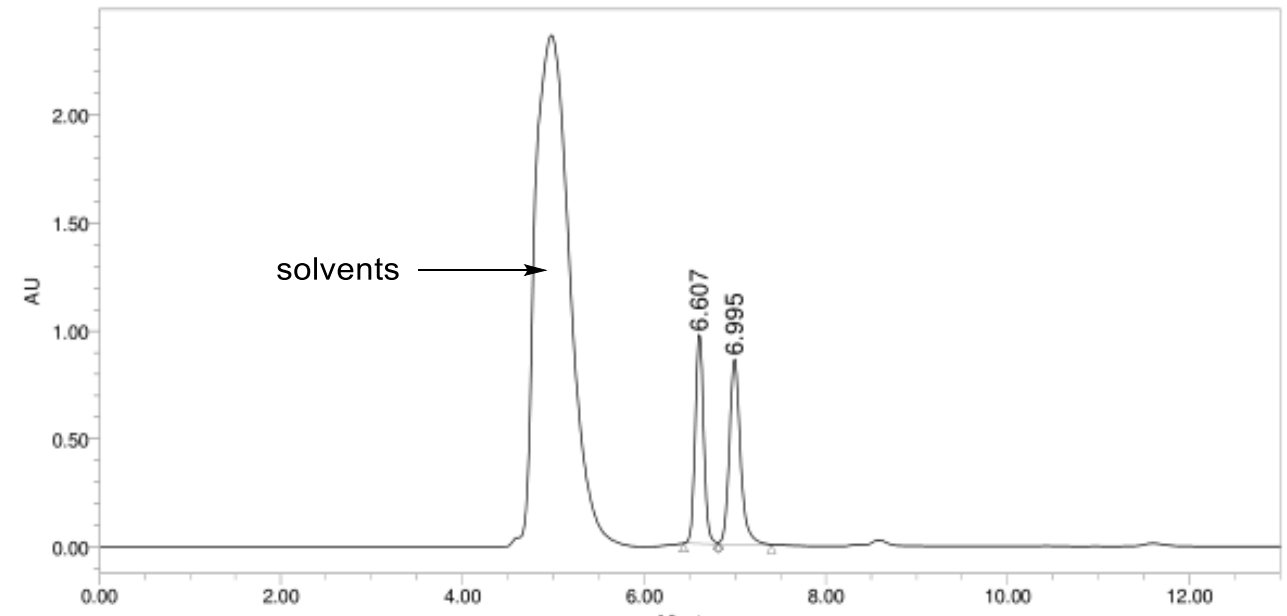


Name: ak_821

Column: Chiralpak IC $4.6 \mathrm{~mm} \times 250 \mathrm{~mm}, 5$ um

Eluent: $10 \%$ MTBE, $90 \%$ Heptane

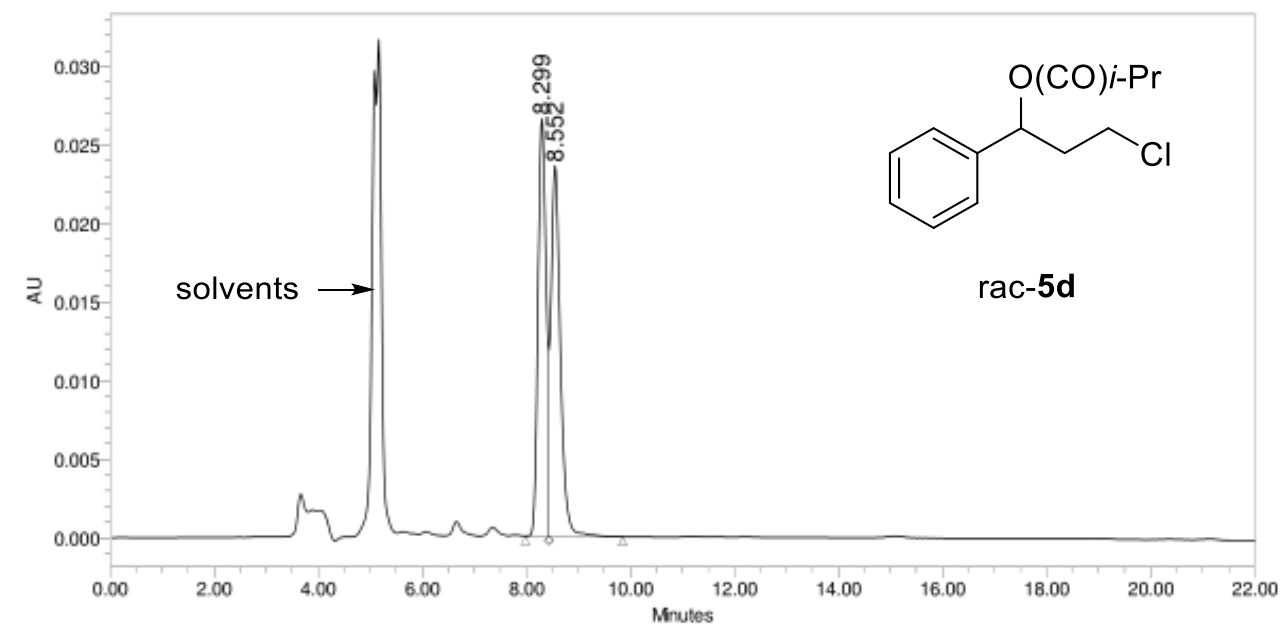

\begin{tabular}{|c|c|c|c|r|}
\hline & RT & Height & Area & $\%$ Area \\
\hline 1 & 8.299 & 26589 & 285047 & 48.68 \\
\hline 2 & 8.552 & 23594 & 300538 & 51.32 \\
\hline
\end{tabular}
Name: ak_845_IC_10\%MTBE 1.0

Column: Chiralpak IC, $4.6 \mathrm{~mm} \times 250 \mathrm{~mm}, 5 \mathrm{um}$

Sample: $1 \mathrm{mg} / \mathrm{mL}$ in 10\% MTBE in Hept

Eluent: $10 \%$ MTBE, $90 \%$ Hept

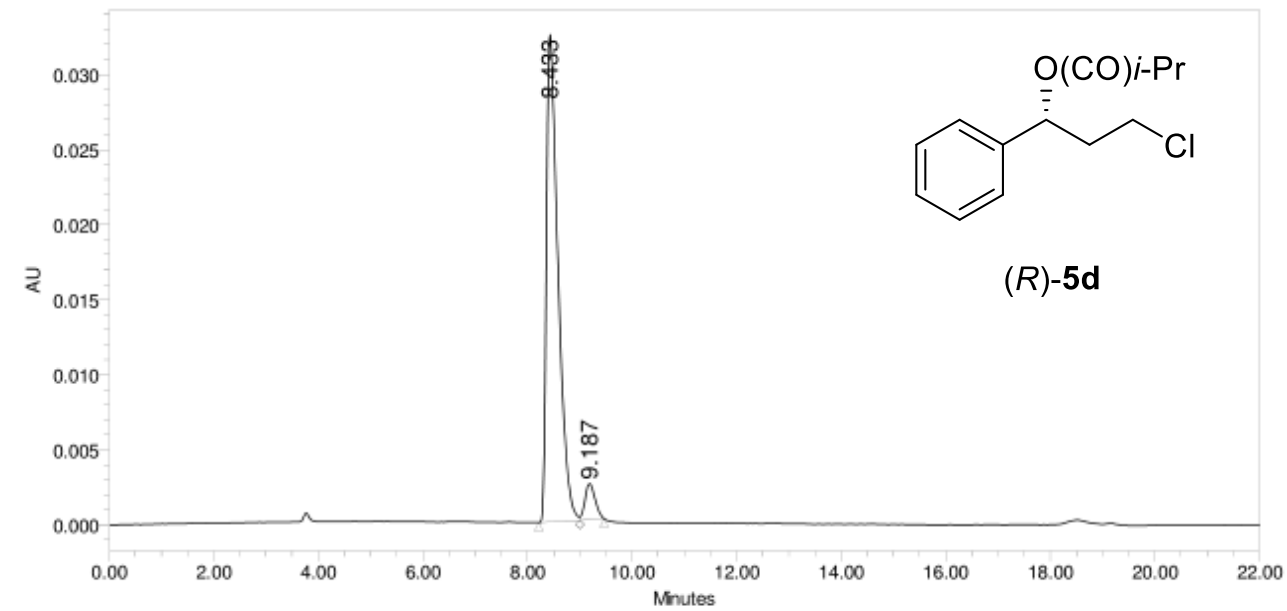

\begin{tabular}{|l|c|r|r|r|}
\hline & RT & Height & Area & \% Area \\
\hline 1 & 8.433 & 32465 & 500120 & 94.02 \\
\hline 2 & 9.187 & 2395 & 31800 & 5.98 \\
\hline
\end{tabular} 
Name: ak_970_rac

k IC $4.6 \mathrm{~mm} \times 250 \mathrm{~mm}, 5 \mathrm{um}$

Flow: $\quad 1.0 \mathrm{~mL} / \mathrm{min}$
Eluent: $5 \%$ IPA, $95 \%$ Heptan

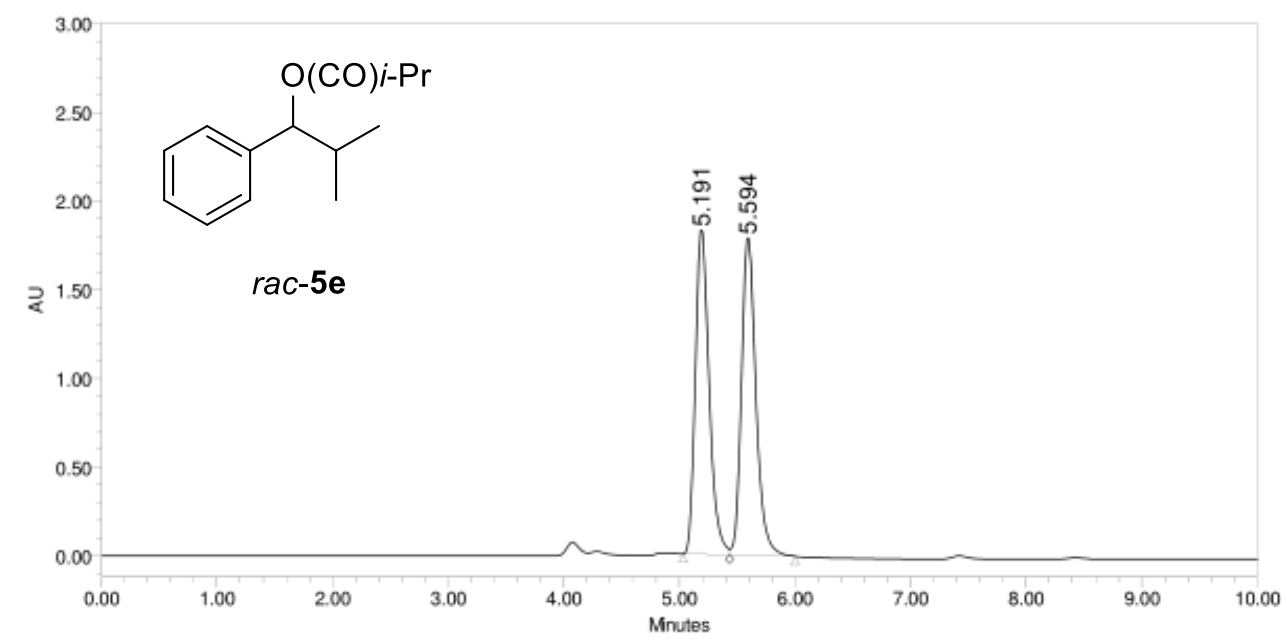

\begin{tabular}{|c|c|c|c|r|}
\hline & RT & Height & Area & \% Area \\
\hline 1 & 5.191 & 1842924 & 15149915 & 49.38 \\
\hline 2 & 5.594 & 1797073 & 15530695 & 50.62 \\
\hline
\end{tabular}

Name: ak_970

Column: Chiralpak IC $4.6 \mathrm{~mm} \times 250 \mathrm{~mm}, 5$ um

Eluent: 5\% IPA, 95\% Heptane

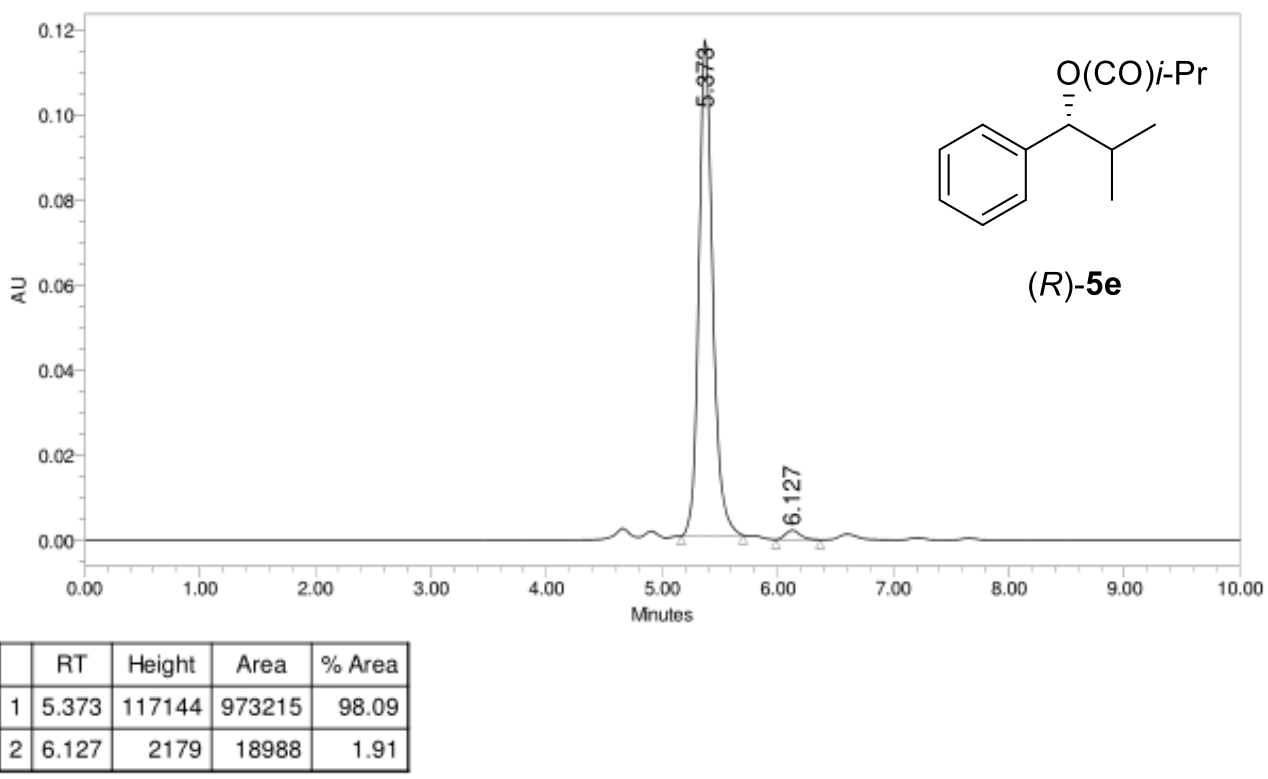


Name: ak_829

Column: Chiralpak IC $4.6 \mathrm{~mm} \times 250 \mathrm{~mm}, 5 \mathrm{um}$

Flow: $1.0 \mathrm{~mL} / \mathrm{min}$

. $2 \%$ IPA, $98 \%$ Heptane
Name: ak_840

Column: Chiralpak IC $4.6 \mathrm{~mm}$ x $250 \mathrm{~mm}, 5 \mathrm{um}$

Flow: $1.0 \mathrm{~mL} / \mathrm{min}$

Eluent: $2 \%$ IPA, $98 \%$ Heptane
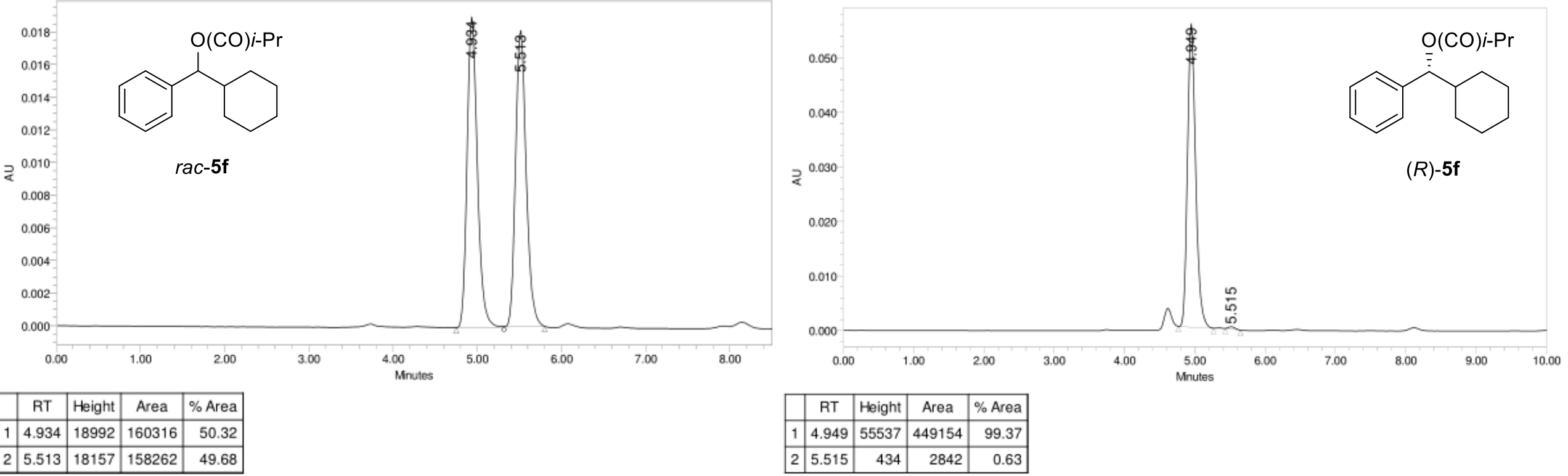
Name: ak_972

Column: Chiralpak IG $4.6 \mathrm{~mm}$ x $250 \mathrm{~mm}, 5$ um

Flow: $1.0 \mathrm{~mL} / \mathrm{min}$

Elont 2\% IPA, 98\% Heptan



Name: ak_977

Column: Chiralpak IG $4.6 \mathrm{~mm}$ x $250 \mathrm{~mm}, 5$ um

Flow: $\quad 1.0 \mathrm{~mL} / \mathrm{min}$
Eluent: $2 \%$ IPA, $98 \%$ Heptane

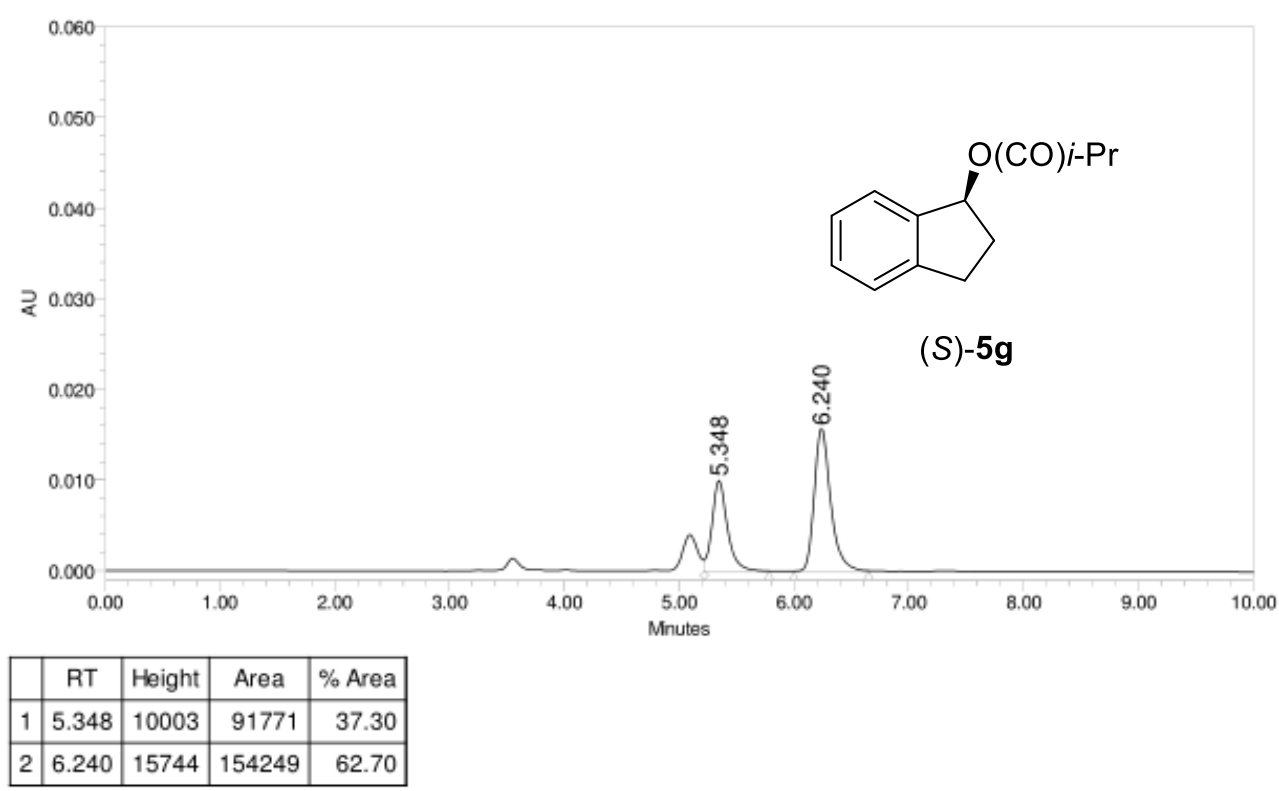


Name: ak_869

Chiralpak IG $4.6 \mathrm{~mm} \times 250 \mathrm{~mm}, 5$ um

Flow. $1.0 \mathrm{~mL} / \mathrm{min}$

Eluent: $5 \%$ IPA, 95\% Heptane

Name: ak_866

Column: Chiralpak IG $4.6 \mathrm{~mm} \times 250 \mathrm{~mm}, 5 \mathrm{um}$

Flow: $\quad 1.0 \mathrm{~mL} / \mathrm{min}$
Eluent: $5 \%$ IPA $95 \%$ Heptan
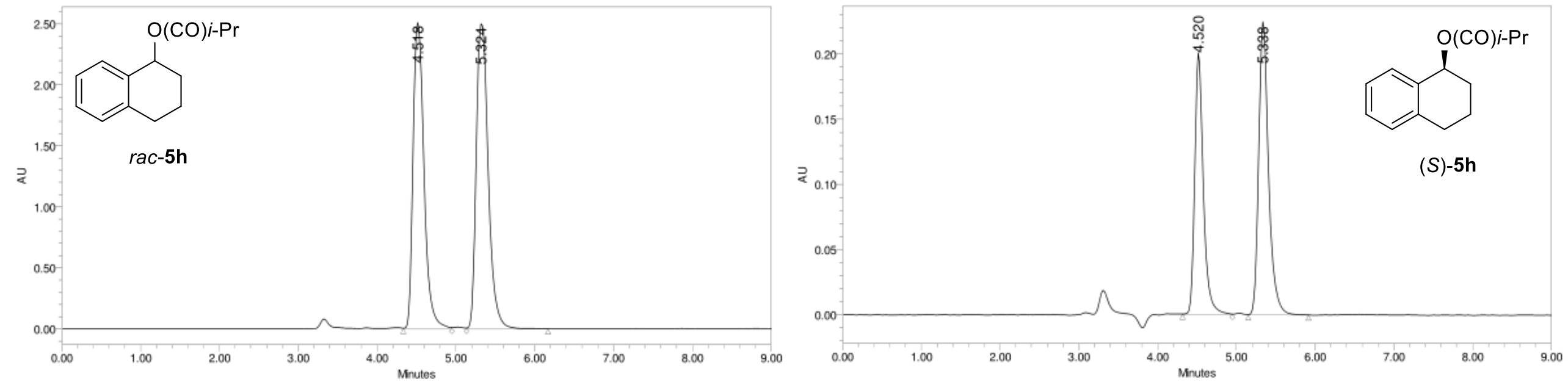

\begin{tabular}{|c|c|c|c|r|}
\hline & RT & Height & Area & \% Area \\
\hline 1 & 4.518 & 2510303 & 24135106 & 47.78 \\
\hline 2 & 5.324 & 2511880 & 26378960 & 52.22 \\
\hline
\end{tabular}

RT Height Area $\%$ Area

\begin{tabular}{|l|l|l|l|l|l|}
\hline 1 & 4.520 & 200125 & 1529583 & 43.82 \\
\hline
\end{tabular}

\begin{tabular}{|l|l|l|l|l|l|}
\hline 2 & 5.338 & 225044 & 1961300 & 56.18 \\
\hline
\end{tabular} 
Name: ak_875

Column: Chiralpak IG $4.6 \mathrm{~mm} \times 250 \mathrm{~mm}, 5 \mathrm{um}$

Feptane

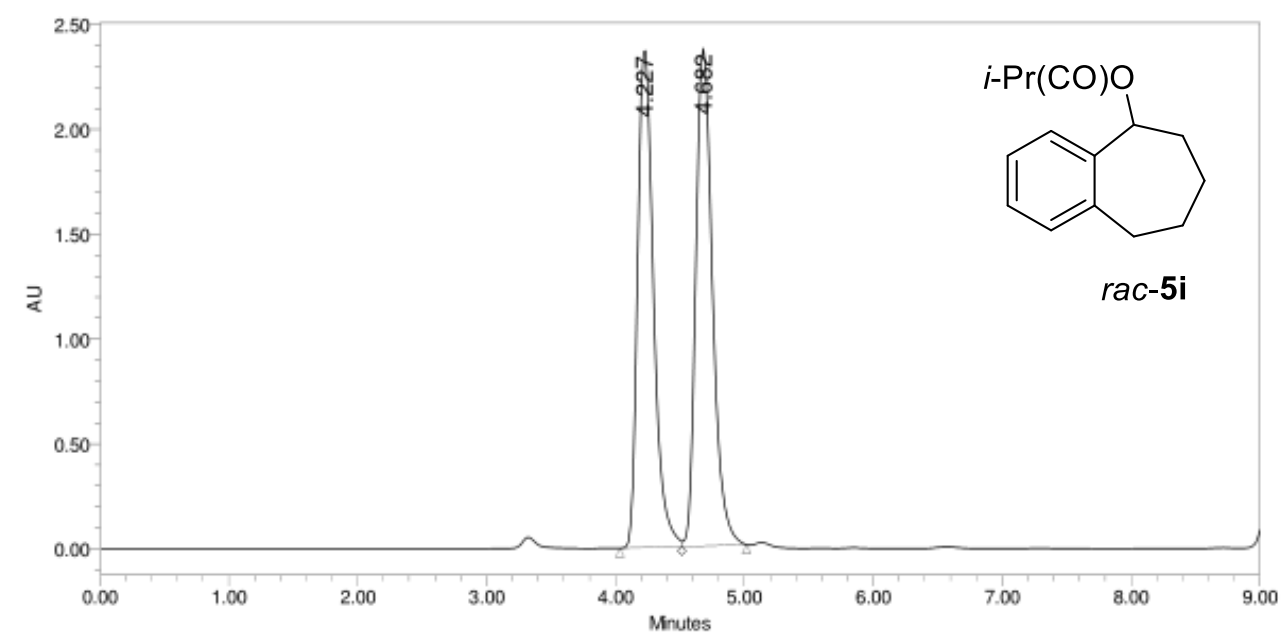

\begin{tabular}{|c|c|c|c|r|}
\hline & RT & Height & Area & \% Area \\
\hline 1 & 4.227 & 2380930 & 20313956 & 48.84 \\
\hline 2 & 4.682 & 2373922 & 21280631 & 51.16 \\
\hline
\end{tabular}

Name: ak_871

Colum: Cralpak IG $4.6 \mathrm{~mm} \times 250 \mathrm{~mm}, 5 \mathrm{um}$

Eluent: 5\% IPA, 95\% Heptane




Name: ak_830

Folum: Chipalpak IC $4.6 \mathrm{~mm} \times 250 \mathrm{~mm}, 5$ um

$\begin{array}{ll}\text { Flow. } & 1.0 \mathrm{~mL} / \mathrm{min} \\ \text { Eluent: } & 10 \% \mathrm{MTBE} 90 \% \text { Heptane }\end{array}$
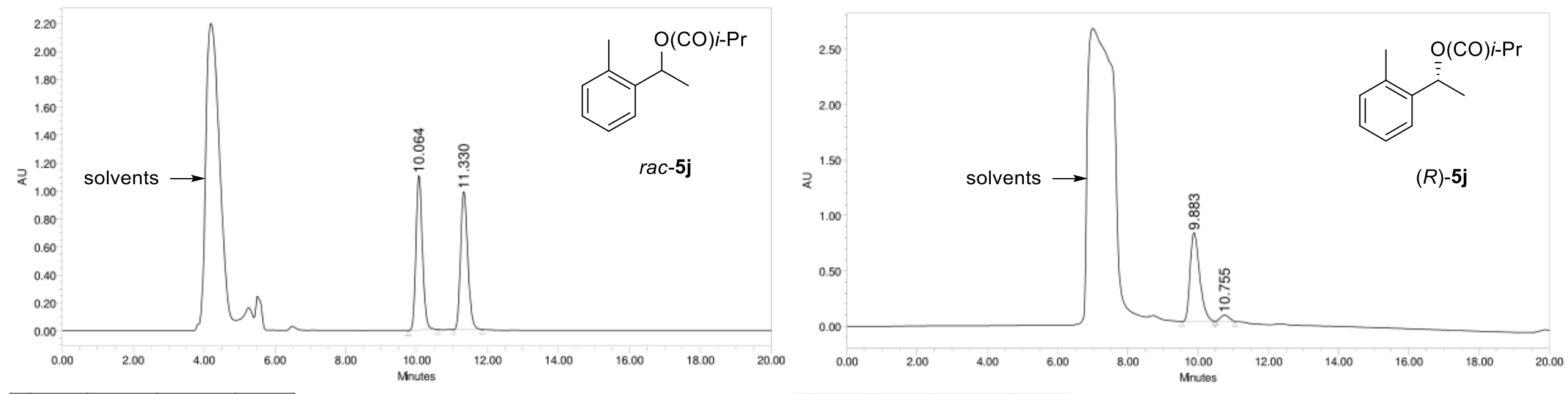

Name: ak_849

Column: Chiralpak IC $4.6 \mathrm{~mm}$ x $250 \mathrm{~mm}, 5$ um

Eluent: $10 \%$ MTBE, $90 \%$ Heptane

\begin{tabular}{|l|r|r|r|r|}
\hline & \multicolumn{1}{|c|}{ RT } & Height & \multicolumn{1}{c|}{ Area } & $\%$ Area \\
\hline 1 & 9.883 & 802875 & 15233835 & 93.86 \\
\hline 2 & 10.755 & 56931 & 997275 & 6.14 \\
\hline
\end{tabular}


Name: ak_831

Column: Chiralpak IC $4.6 \mathrm{~mm} \times 250 \mathrm{~mm}, 5 \mathrm{um}$

$\begin{array}{ll}\text { Flow. } & 1.0 \mathrm{~mL} / \mathrm{min} \\ \text { Eluent: } & 10 \% \mathrm{MTBE}, 90 \% \text { Heptane }\end{array}$
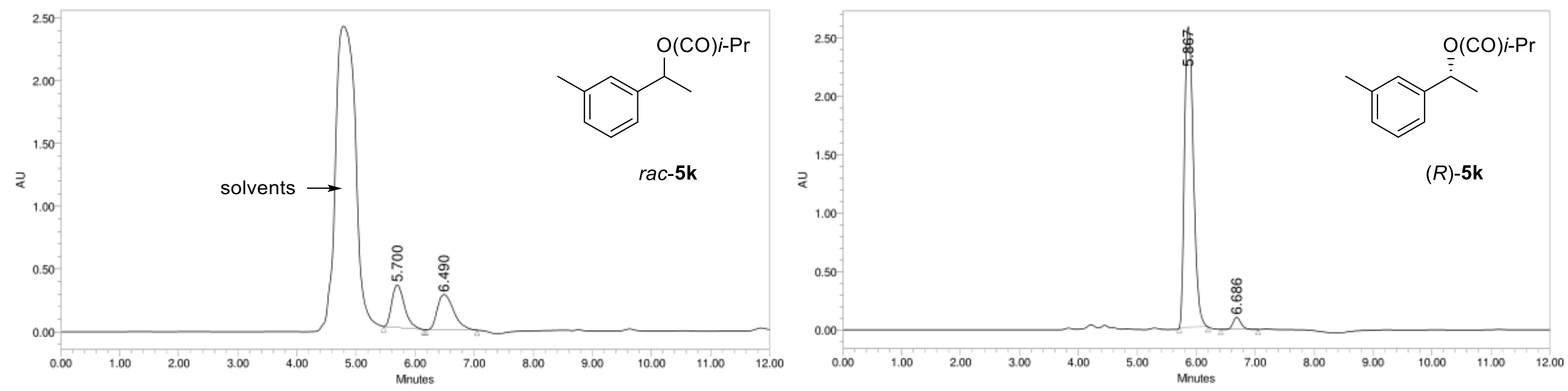

\begin{tabular}{|c|c|c|c|r|}
\hline & RT & Height & Area & \% Area \\
\hline 1 & 5.700 & 339471 & 5045158 & 48.14 \\
\hline 2 & 6.490 & 280274 & 5435955 & 51.86 \\
\hline
\end{tabular}
Name: ak_848

.

Eluent: $10 \% \mathrm{MT}$ min

\begin{tabular}{|r|c|r|r|r|}
\hline & RT & Height & \multicolumn{1}{c|}{ Area } & $\%$ Area \\
\hline 1 & 5.867 & 2577271 & 27098257 & 96.56 \\
\hline 2 & 6.686 & 104126 & 966011 & 3.44 \\
\hline
\end{tabular}


Name: ak 834

Column: Chiralpak IC $4.6 \mathrm{~mm} \times 250 \mathrm{~mm}, 5 \mathrm{um}$

Eluent: $5 \%$ IPA, $95 \%$ Heptan



\begin{tabular}{|c|c|c|c|r|}
\hline & RT & Height & Area & $\%$ Area \\
\hline 1 & 5.204 & 148461 & 1213654 & 49.94 \\
\hline 2 & 6.878 & 122503 & 1216788 & 50.06 \\
\hline
\end{tabular}

Name: ak_842

Column: Chiralpak IC $4.6 \mathrm{~mm} \times 250 \mathrm{~mm}, 5$ um

Eluent: $5 \%$ IPA, 95\% Heptane

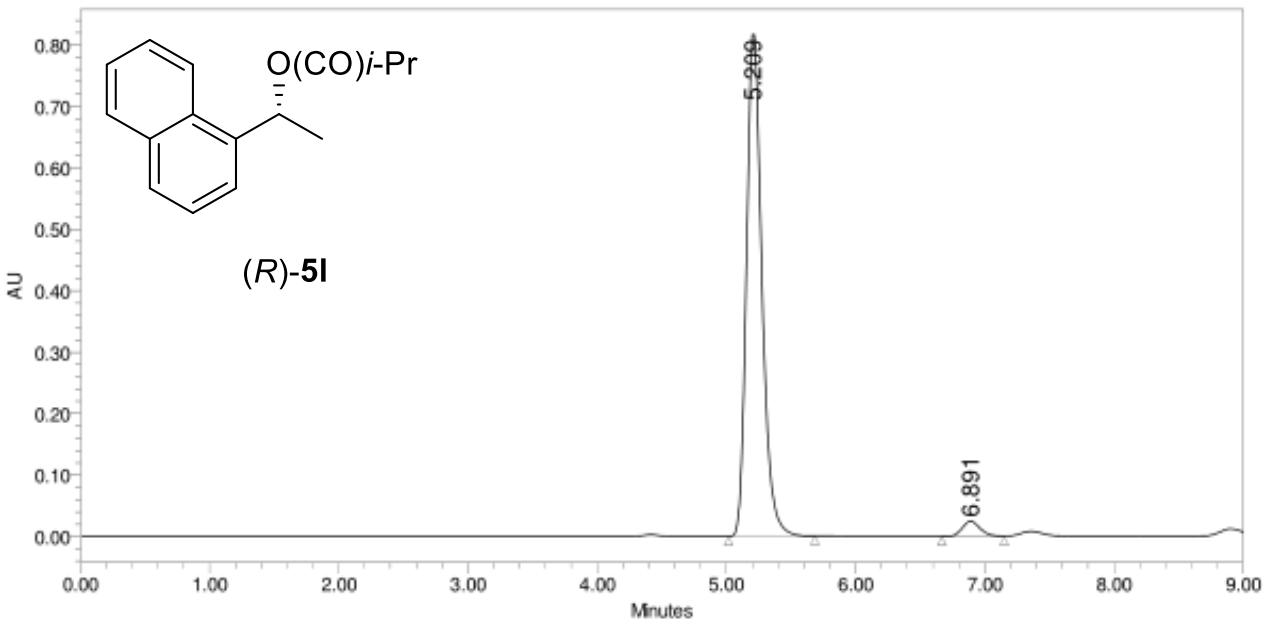

\begin{tabular}{|l|c|r|r|r|}
\hline & RT & Height & Area & $\%$ Area \\
\hline 1 & 5.209 & 824567 & 6770210 & 96.55 \\
\hline 2 & 6.891 & 24851 & 242190 & 3.45 \\
\hline
\end{tabular} 
Name: ak_835

Column: Chiralpak IC $4.6 \mathrm{~mm} \times 250 \mathrm{~mm}, 5$ um

Flow. $\quad 1.0 \mathrm{~mL} / \mathrm{min}$
Eluent: $5 \%$ PPA, $95 \%$ Heptane
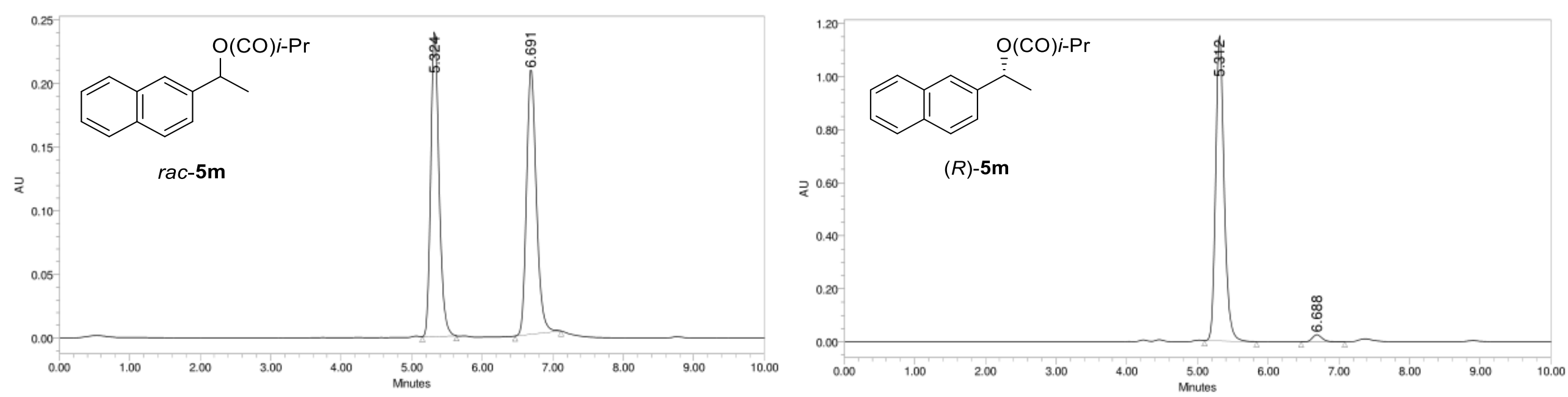

\begin{tabular}{|c|c|c|c|r|}
\hline & RT & Height & Area & $\%$ Area \\
\hline 1 & 5.324 & 240691 & 1964849 & 49.40 \\
\hline 2 & 6.691 & 209000 & 2012797 & 50.60 \\
\hline
\end{tabular}
Name: ak_843

Colum Chiralpak IC $4.6 \mathrm{~mm} \times 250 \mathrm{~mm}$, 5 um

Eluent: 5\% IPA, 95\% Heptane

\begin{tabular}{|c|c|r|r|r|}
\hline & RT & \multicolumn{1}{c|}{ Height } & Area & \% Area \\
\hline 1 & 5.312 & 1153090 & 9645604 & 97.38 \\
\hline 2 & 6.688 & 27010 & 259384 & 2.62 \\
\hline
\end{tabular}


Name: ak_876

pak IG $4.6 \mathrm{~mm} \times 250 \mathrm{~mm}, 5 \mathrm{um}$

Eluent: $0.5 \%$ IPA $99.5 \%$ Heptane

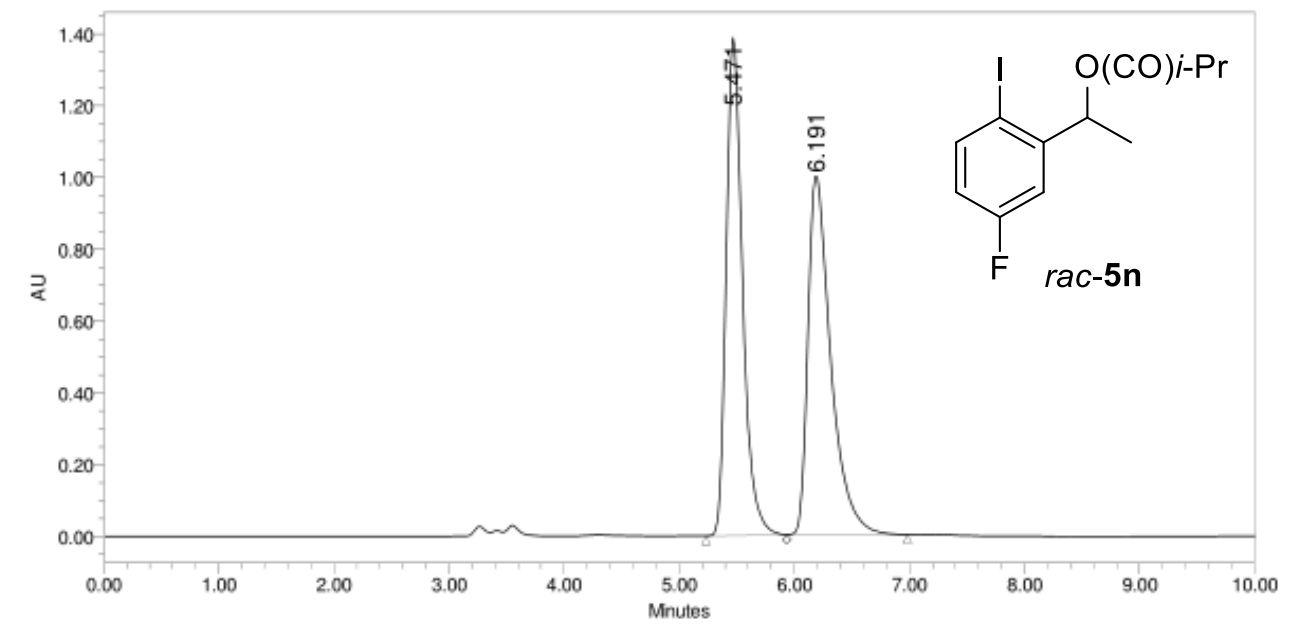

\begin{tabular}{|c|c|c|c|r|}
\hline & RT & Height & Area & $\%$ Area \\
\hline 1 & 5.471 & 1388512 & 13688566 & 49.78 \\
\hline 2 & 6.191 & 1004747 & 13809478 & 50.22 \\
\hline
\end{tabular}
Name: ak_872

Column: Chiralpak IG $4.6 \mathrm{~mm} \times 250 \mathrm{~mm}, 5$ um

Eluent: $0.5 \%$ IPA $99.5 \%$ Heptane

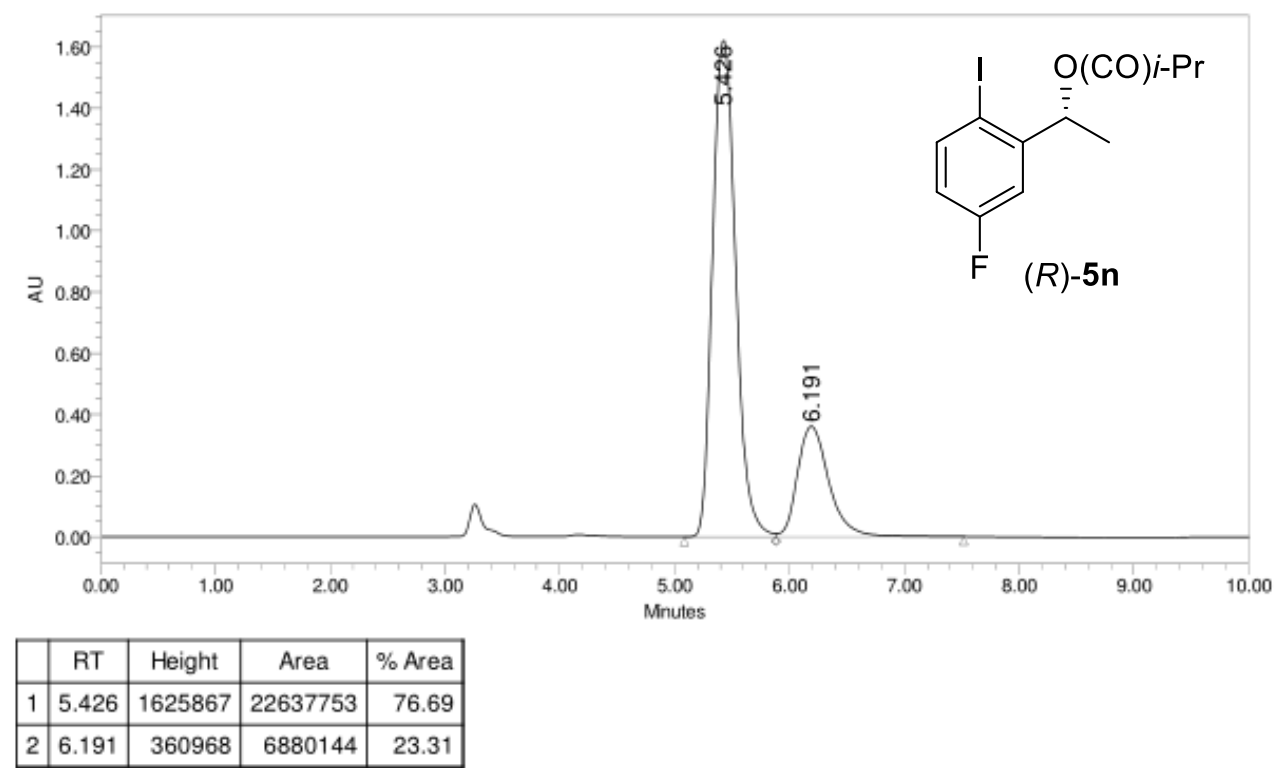


Name: ak_828

Column: Chiralpak IG $4.6 \mathrm{~mm} \times 250 \mathrm{~mm}, 5 \mathrm{um}$

$1.0 \mathrm{~mL} / \mathrm{min}$

Eluent: $5 \%$ IPA, 95\% Heptane



\begin{tabular}{|c|c|c|c|r|}
\hline & RT & Height & Area & \% Area \\
\hline 1 & 4.856 & 23806 & 192825 & 49.38 \\
\hline 2 & 5.833 & 20763 & 197680 & 50.62 \\
\hline
\end{tabular}
Name: ak_937

Column: Chiralpak IG $4.6 \mathrm{~mm} \times 250 \mathrm{~mm}, 5 \mathrm{um}$

Eluent: $5 \%$ IPA, $95 \%$ Heptane

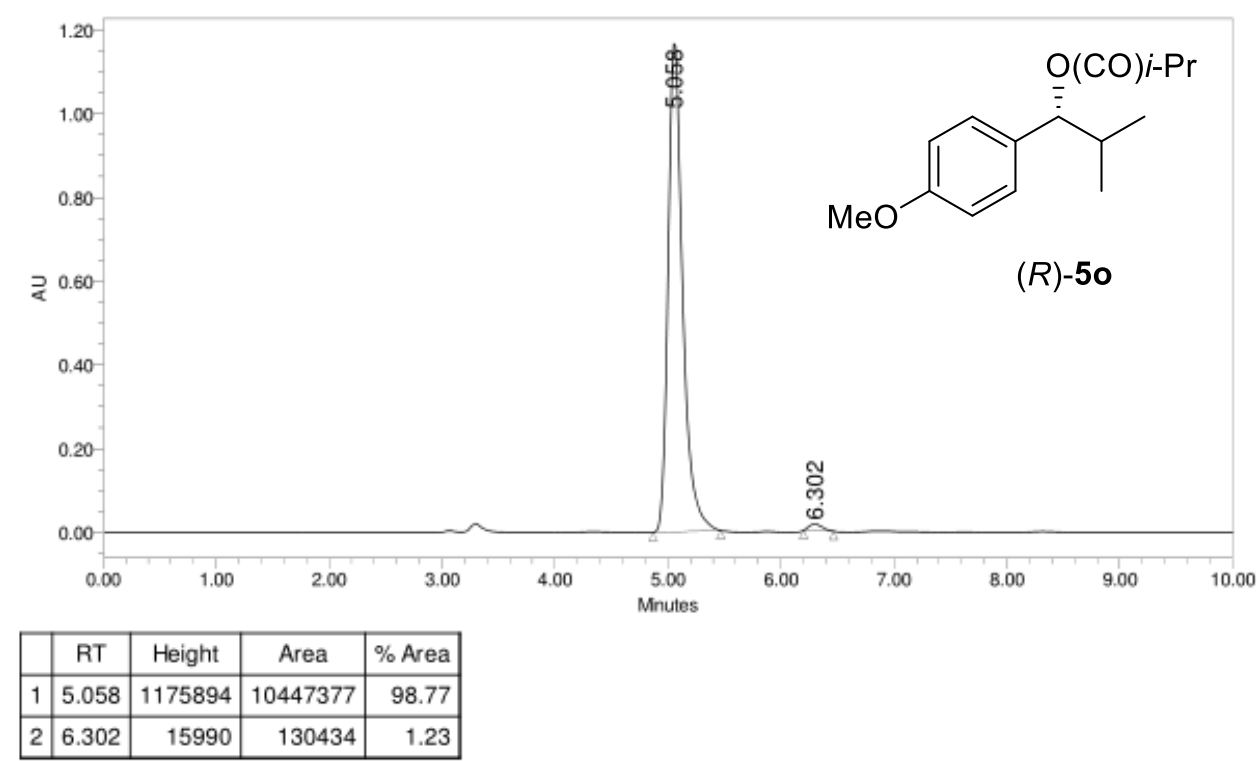


Name: ak_827

Collm Chiralpak IC $4.6 \mathrm{~mm} \times 250 \mathrm{~mm}, 5$ um

Fluent: $10 \%$ MTBE $90 \%$ Heptane

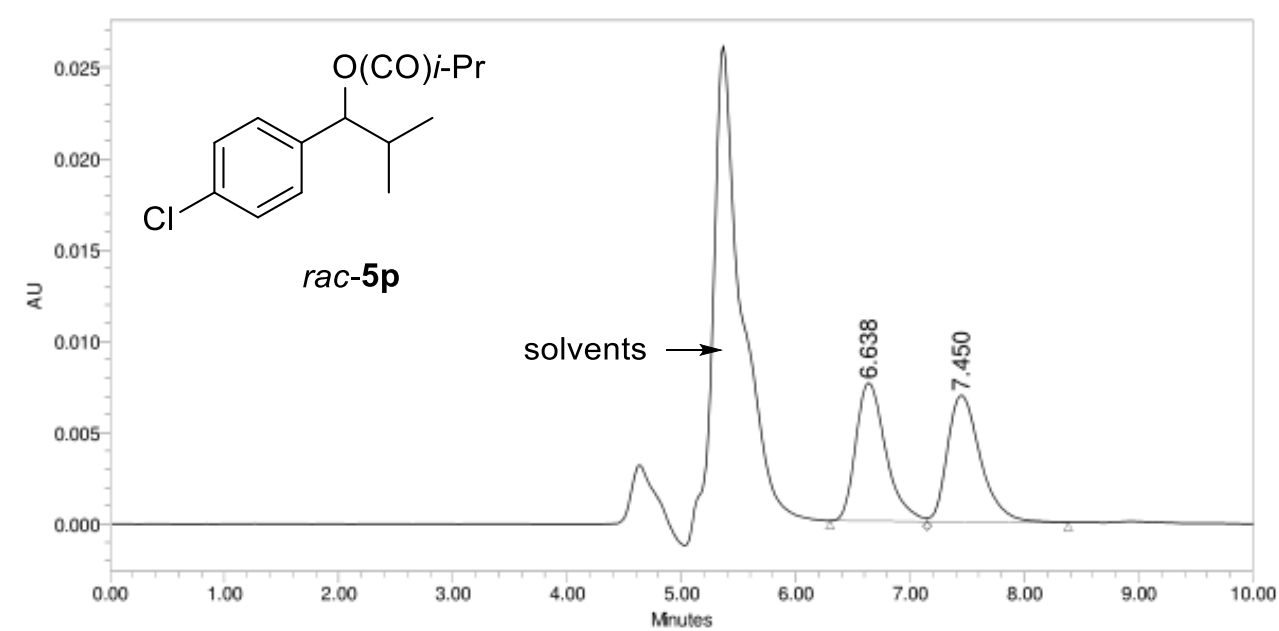

\begin{tabular}{|c|c|r|c|r|}
\hline & RT & Height & Area & $\%$ Area \\
\hline 1 & 6.638 & 7564 & 138524 & 49.88 \\
\hline 2 & 7.450 & 6922 & 139200 & 50.12 \\
\hline
\end{tabular}
Name: ak_971
Column: Chiralpak IC $4.6 \mathrm{~mm} \times 250 \mathrm{~mm}, 5 \mathrm{um}$

Flow. $1.0 \mathrm{~mL} / \mathrm{min}$.

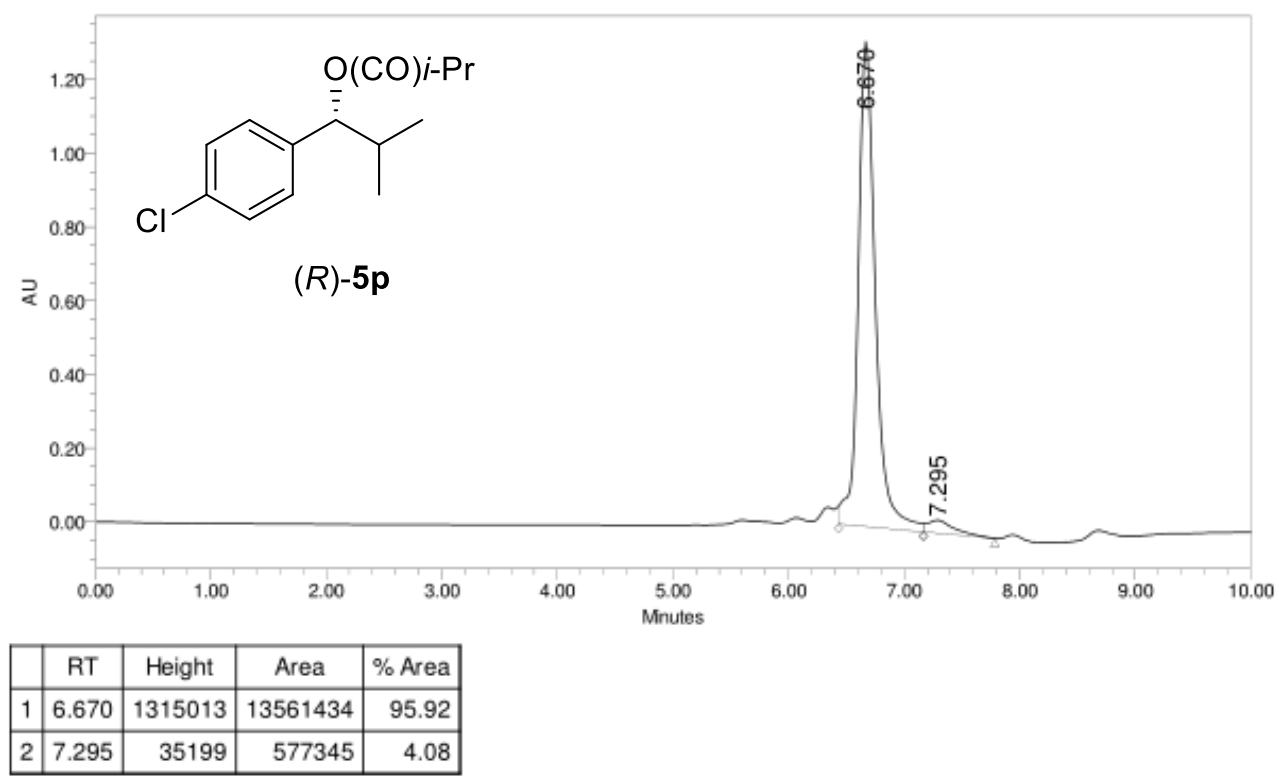


Name: ak_877

Column. Chiralpak IG $4.6 \mathrm{~mm} \times 250 \mathrm{~mm}, 5$ um

Feptane

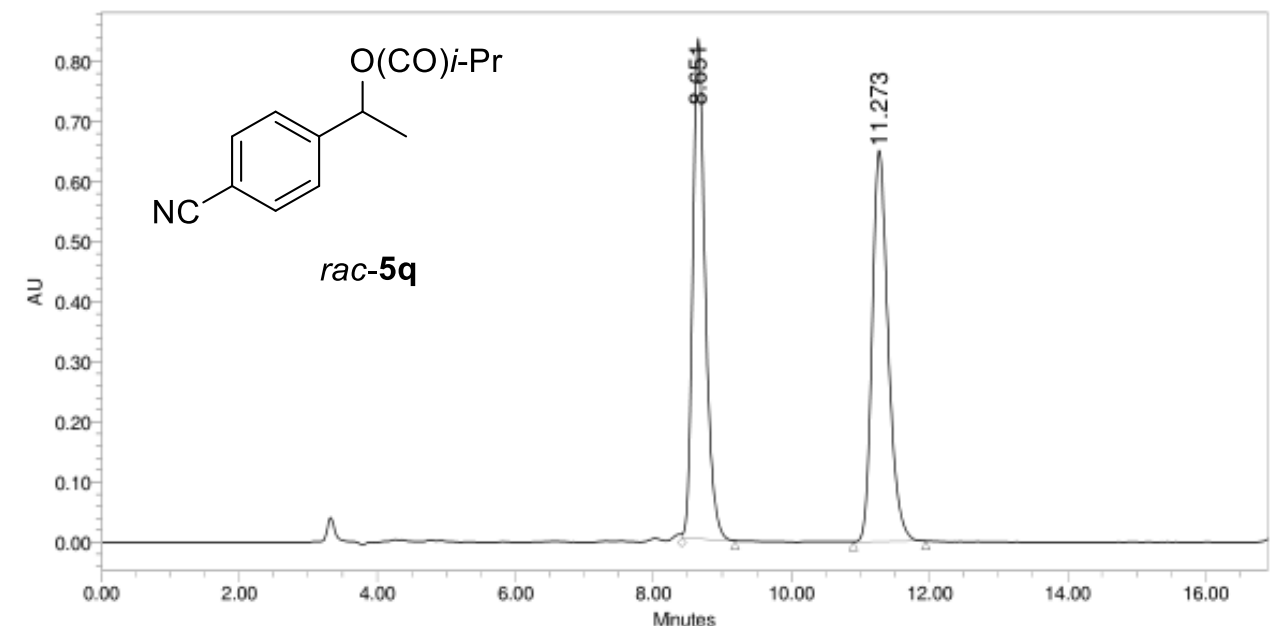

\begin{tabular}{|c|c|c|c|r|}
\hline & \multicolumn{1}{|c|}{ RT } & Height & Area & \% Area \\
\hline 1 & 8.651 & 833112 & 10464247 & 49.92 \\
\hline 2 & 11.273 & 652414 & 10498538 & 50.08 \\
\hline
\end{tabular}

Name: ak_o/s

alpak IG $4.6 \mathrm{~mm} \times 250 \mathrm{~mm}, 5 \mathrm{um}$

Flow. $\quad 1.0 \mathrm{~mL} / \mathrm{min}$
Eluent:
$5 \%$ IPA, $95 \%$ Heptane

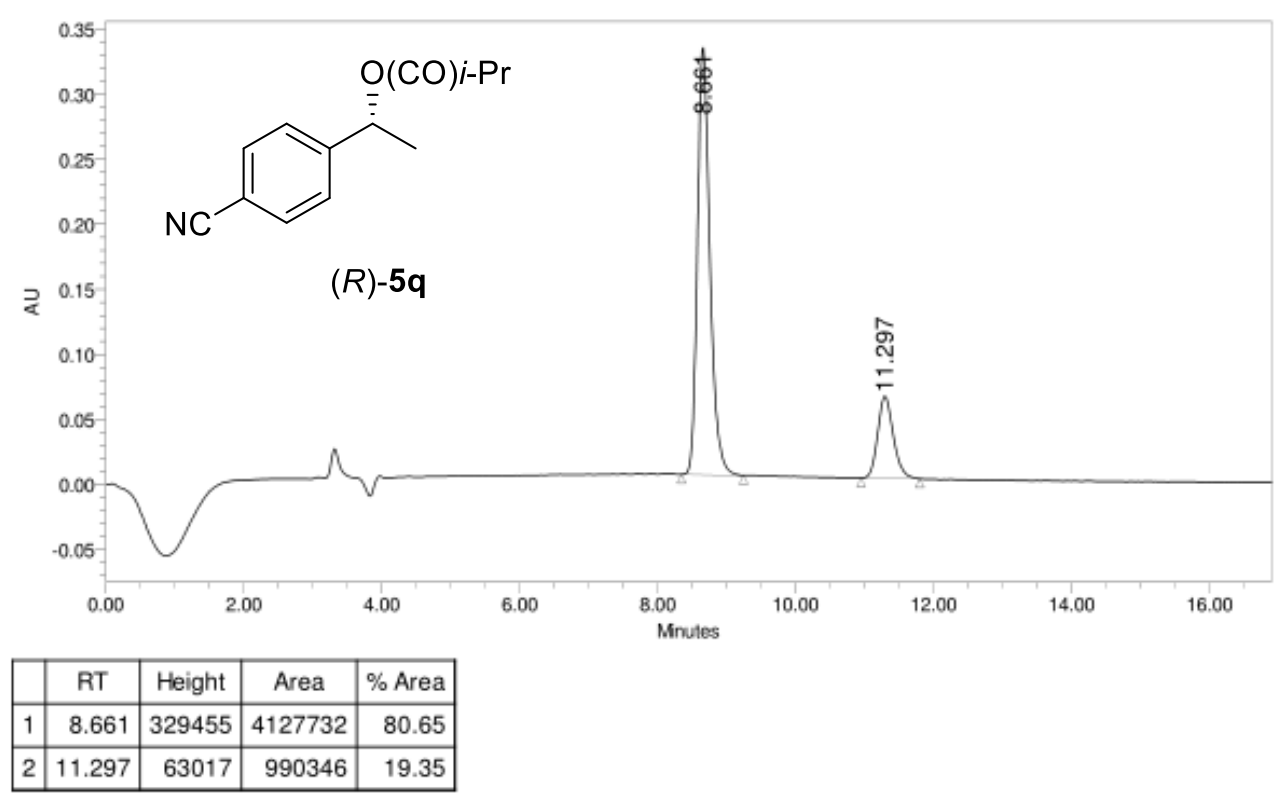


Name: ak_833

Column: Chiralpak IG $4.6 \mathrm{~mm} \times 250 \mathrm{~mm}, 5$ um

Cow. $1.0 \mathrm{~mL}$ min

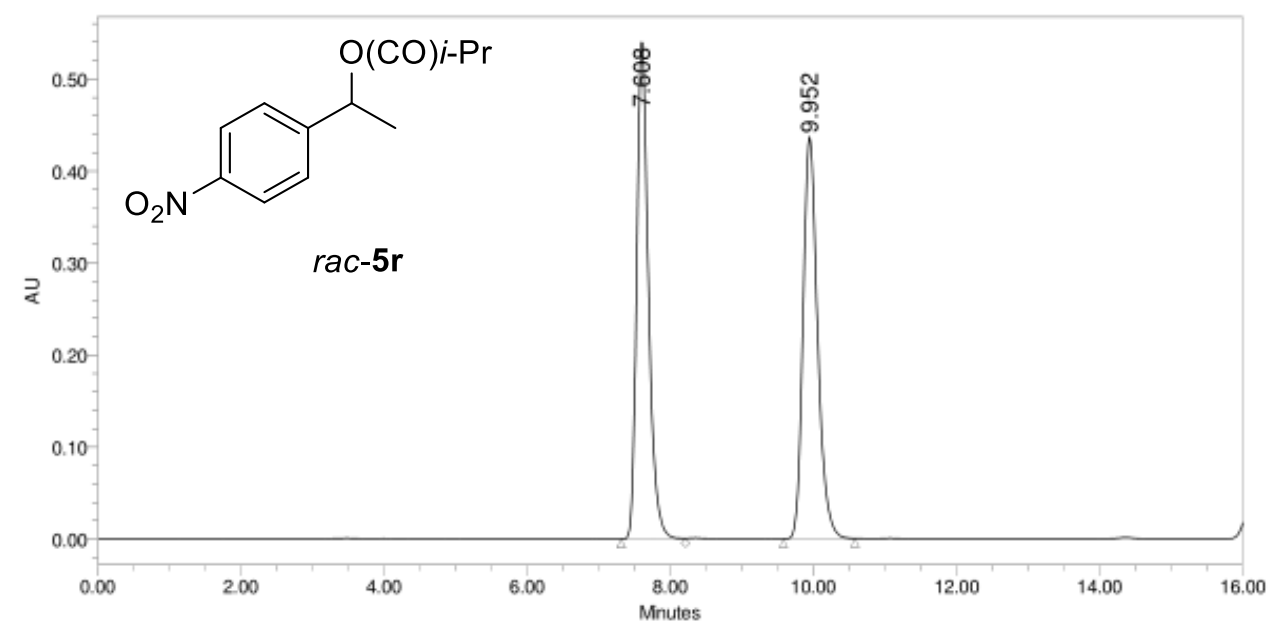

\begin{tabular}{|c|c|c|c|r|}
\hline & RT & Height & Area & $\%$ Area \\
\hline 1 & 7.608 & 543308 & 6171170 & 50.02 \\
\hline 2 & 9.952 & 437197 & 6165596 & 49.98 \\
\hline
\end{tabular}
Name: ak_841

Column: Chiralpak IG $4.6 \mathrm{~mm} \times 250 \mathrm{~mm}, 5$ um

Eluent: $5 \%$ IPA $95 \%$ Heptane

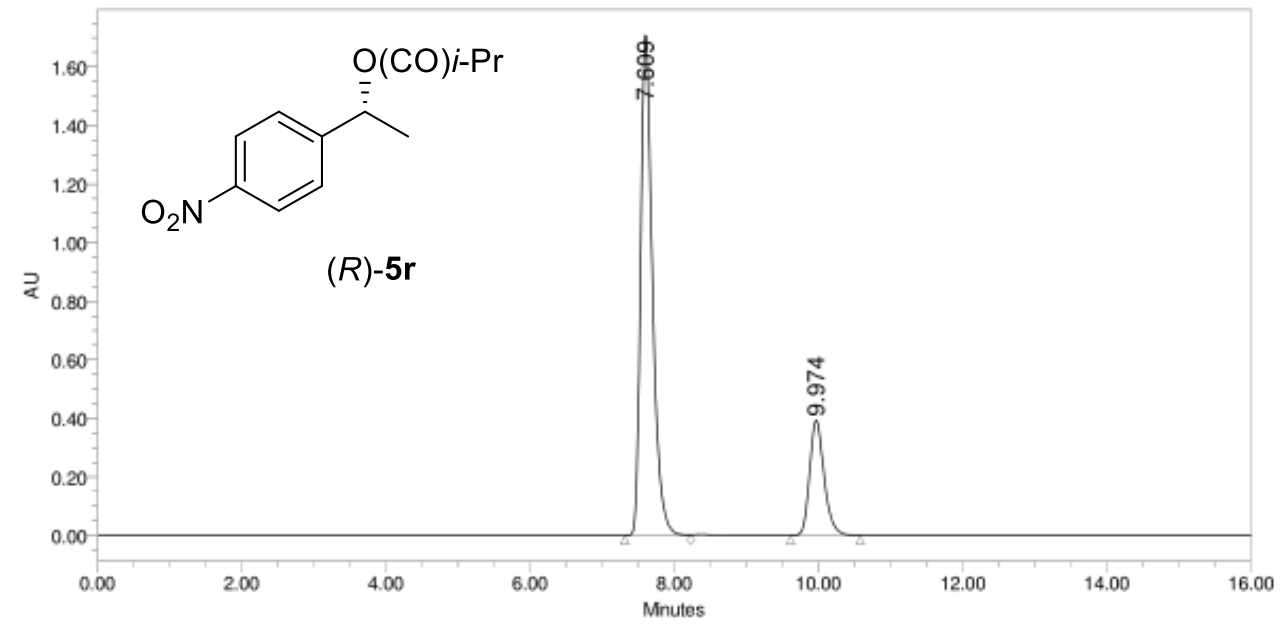

\begin{tabular}{|c|c|c|r|r|}
\hline & RT & Height & \multicolumn{1}{c|}{ Area } & $\%$ Area \\
\hline 1 & 7.609 & 1720811 & 19493263 & 78.05 \\
\hline 2 & 9.974 & 393250 & 5482533 & 21.95 \\
\hline
\end{tabular} 
Name: ak_906

Column. Chiralpak IG $4.6 \mathrm{~mm} \times 250 \mathrm{~mm}, 5 \mathrm{um}$

Eluent: $5 \%$ IPA, 95\% Heptane

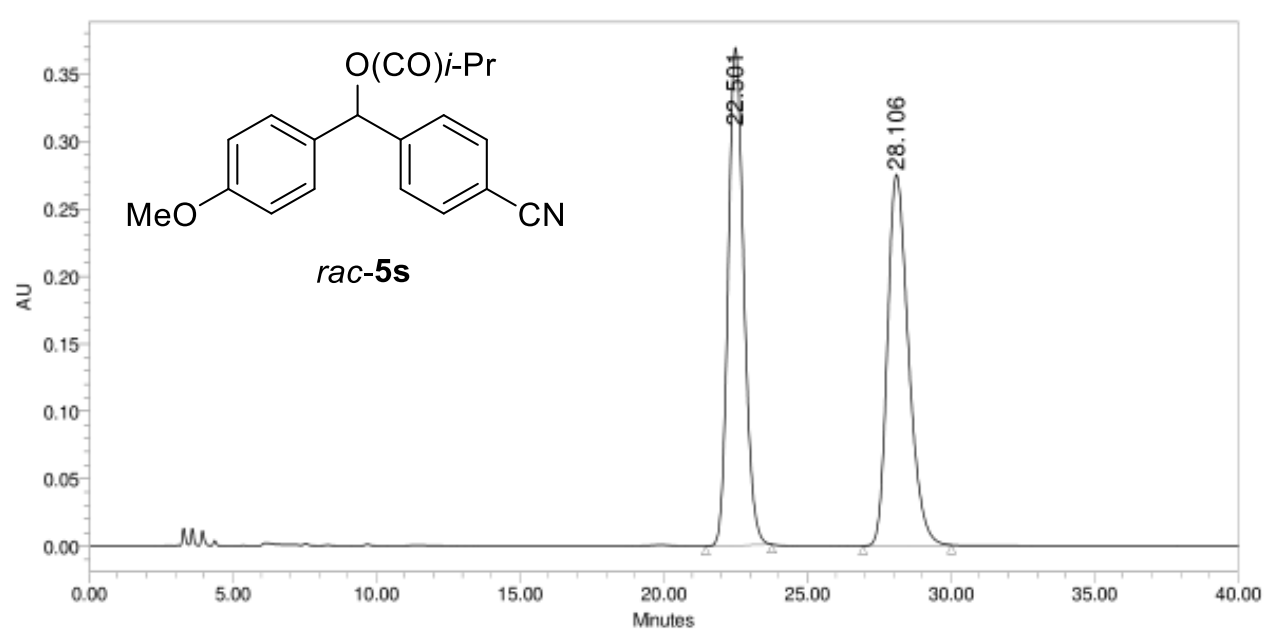

\begin{tabular}{|c|c|c|c|r|}
\hline & RT & Height & Area & \% Area \\
\hline 1 & 22.501 & 369000 & 13969473 & 49.98 \\
\hline 2 & 28.106 & 274932 & 13983097 & 50.02 \\
\hline
\end{tabular}

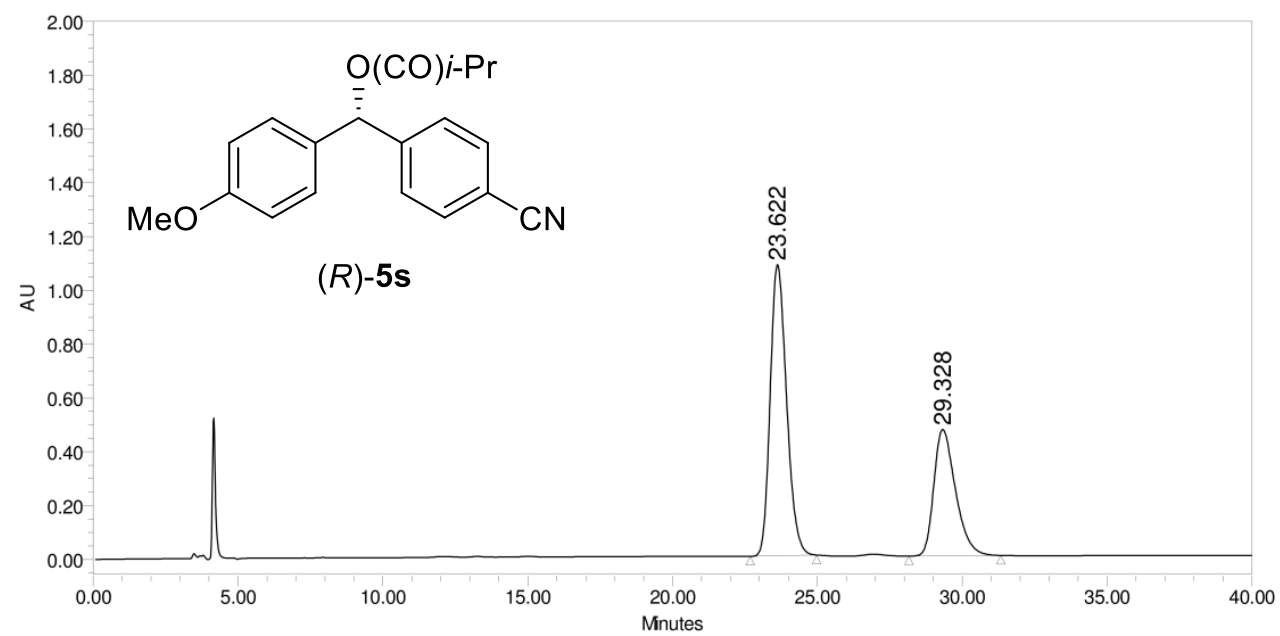

Name: ak 918

Column: Chiralpak IG $4.6 \mathrm{~mm} \times 250 \mathrm{~mm}, 5$ um

$\begin{array}{ll}\text { Flow: } & 1.0 \mathrm{~mL} / \mathrm{min} \\ \text { Eluent: } & 5 \% \mathrm{IPA}, 95 \% \text { Heptan }\end{array}$

\begin{tabular}{|c|c|c|c|r|}
\hline & RT & Height & Area & $\%$ Area \\
\hline 1 & 23.622 & 1084478 & 42888832 & 66.75 \\
\hline 2 & 29.332 & 440018 & 21366053 & 33.25 \\
\hline
\end{tabular} 
Name: Ib_120

Column: Chiralpak IG $4.6 \mathrm{~mm} \times 250 \mathrm{~mm}, 5$ um

Fow.

5\% IPA $95 \%$ Heptan

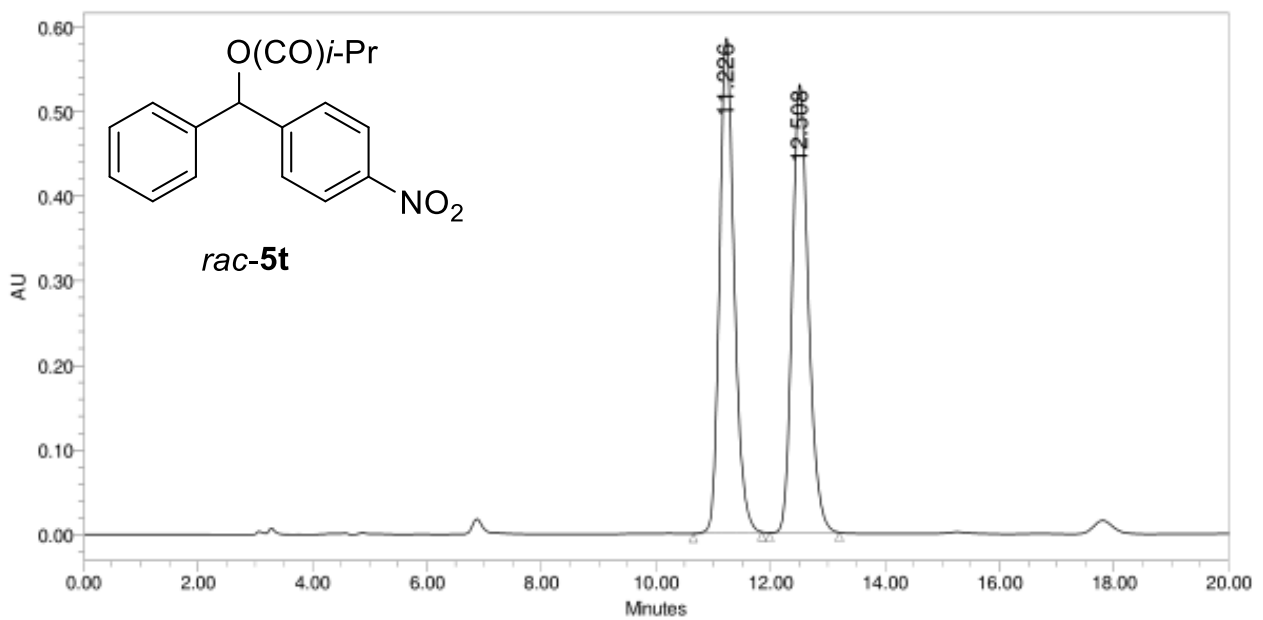

\begin{tabular}{c|c|c|c|r|}
\hline & RT & Height & Area & \% Area \\
\hline 1 & 11.226 & 586480 & 10428052 & 50.13 \\
\hline 2 & 12.508 & 530888 & 10373882 & 49.87 \\
\hline
\end{tabular}
Name: ak_940

Column: Chiralpak IG $4.6 \mathrm{~mm}$ x 250 mm, 5 um

Elow: $1.0 \mathrm{~mL} / \mathrm{min}$
Eluent: $5 \%$ IPA, $95 \%$ Heptane

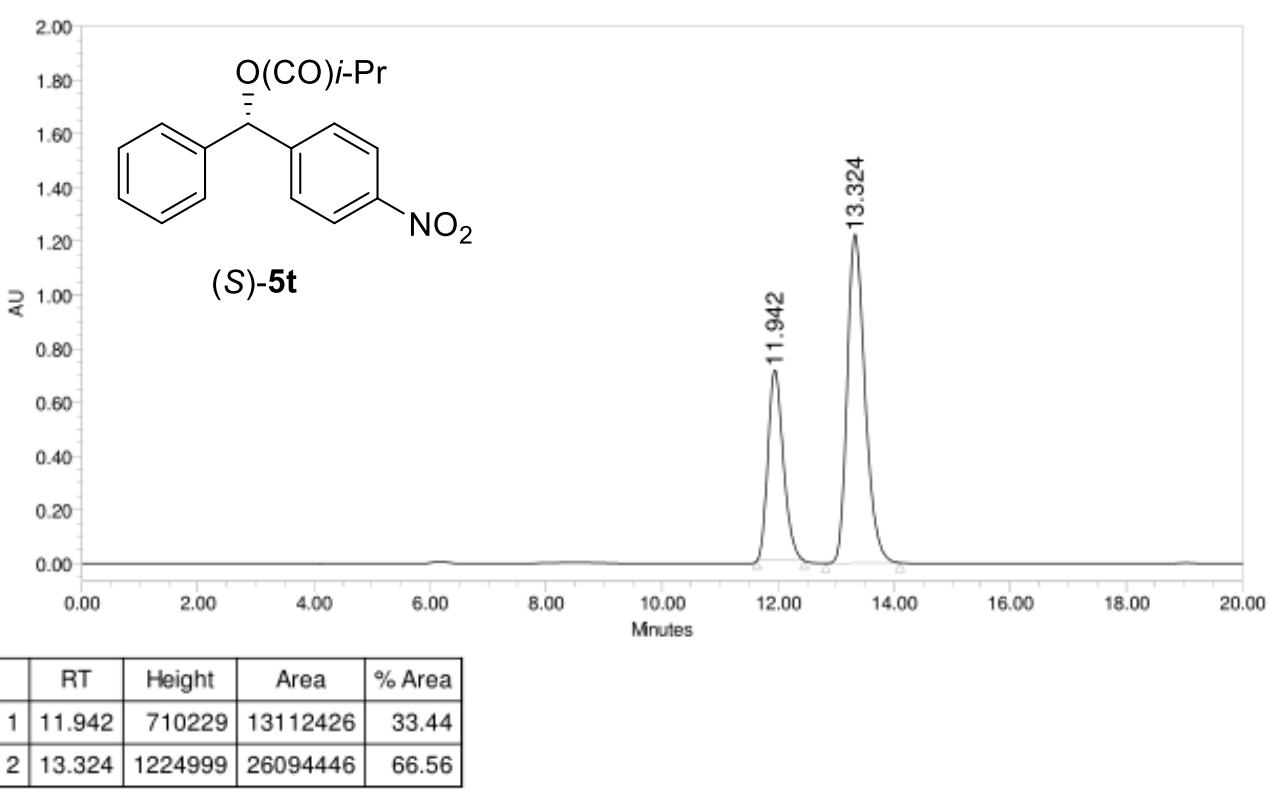


Name: ak 941

Column: Chiralpak IC $4.6 \mathrm{~mm} \times 250 \mathrm{~mm}, 5$ um

Eluent: $2 \%$ IPA, $98 \%$ Heptane



Name: ak_941

Column: Chiralpak IC $4.6 \mathrm{~mm} \times 250 \mathrm{~mm}, 5 \mathrm{um}$

Eluent: $2 \% \mathrm{IPA}, 98 \%$ Heptane




Name: ak_922
Column: Chiralpak IA $4.6 \mathrm{~mm} \times 250 \mathrm{~mm}, 5 \mathrm{um}$

Flow. $1.0 \mathrm{~mL} / \mathrm{min}$
Eluent: $5 \% \mathrm{PA}, 95 \%$ Heptane
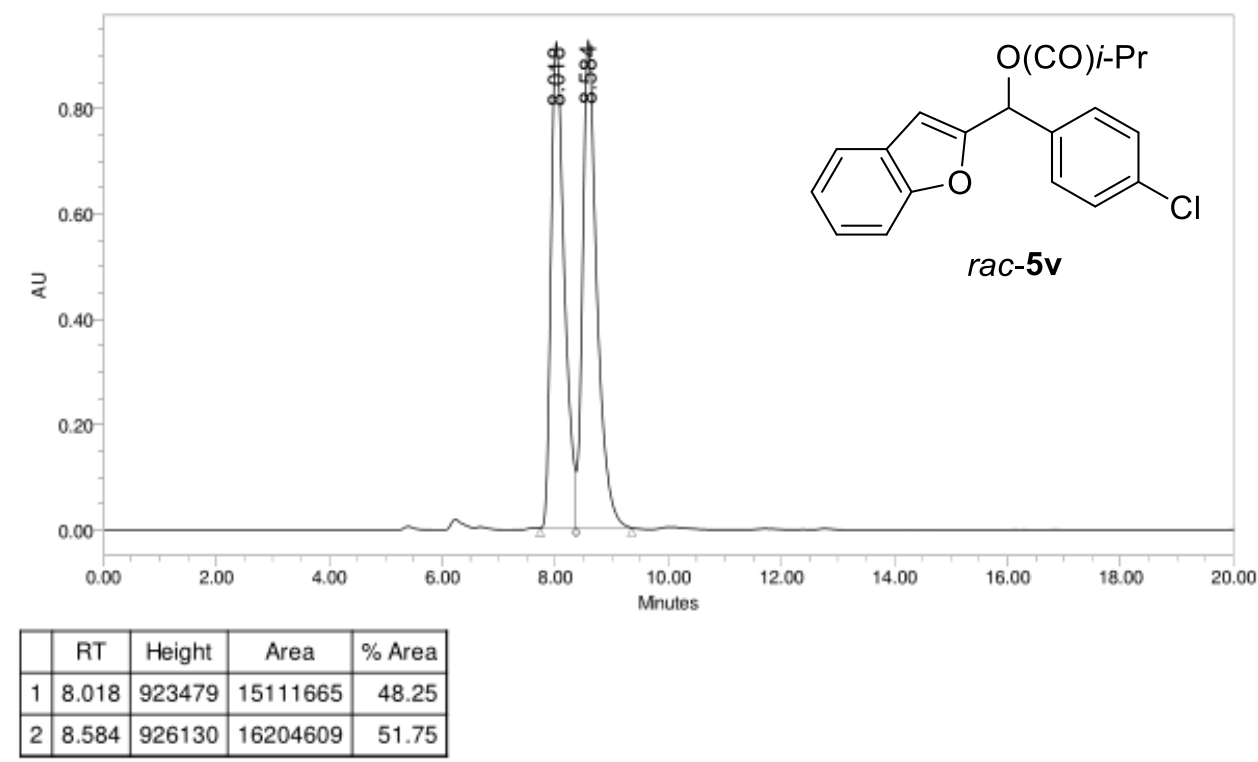

Name: ak_920

Column: Chiralpak IA $4.6 \mathrm{~mm}$ x 250 mm, 5 um

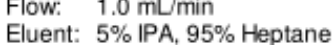

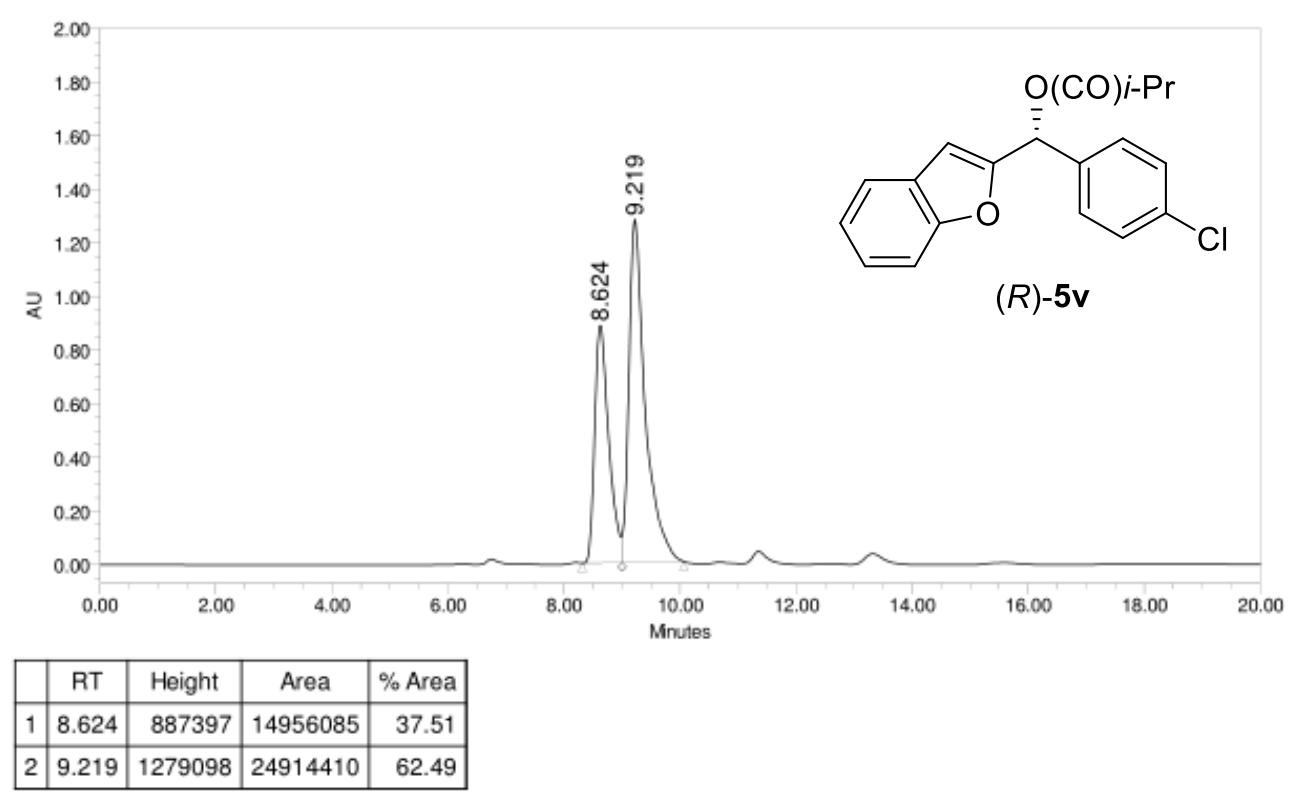


${ }^{1} \mathrm{H}$ NMR, ${ }^{13} \mathrm{C}$ NMR and ${ }^{19} \mathrm{~F}$ NMR data 
${ }^{1} \mathrm{H}$ NMR (300 MHz, $\left.\mathrm{CDCl}_{3}, \mathrm{ppm}\right)$

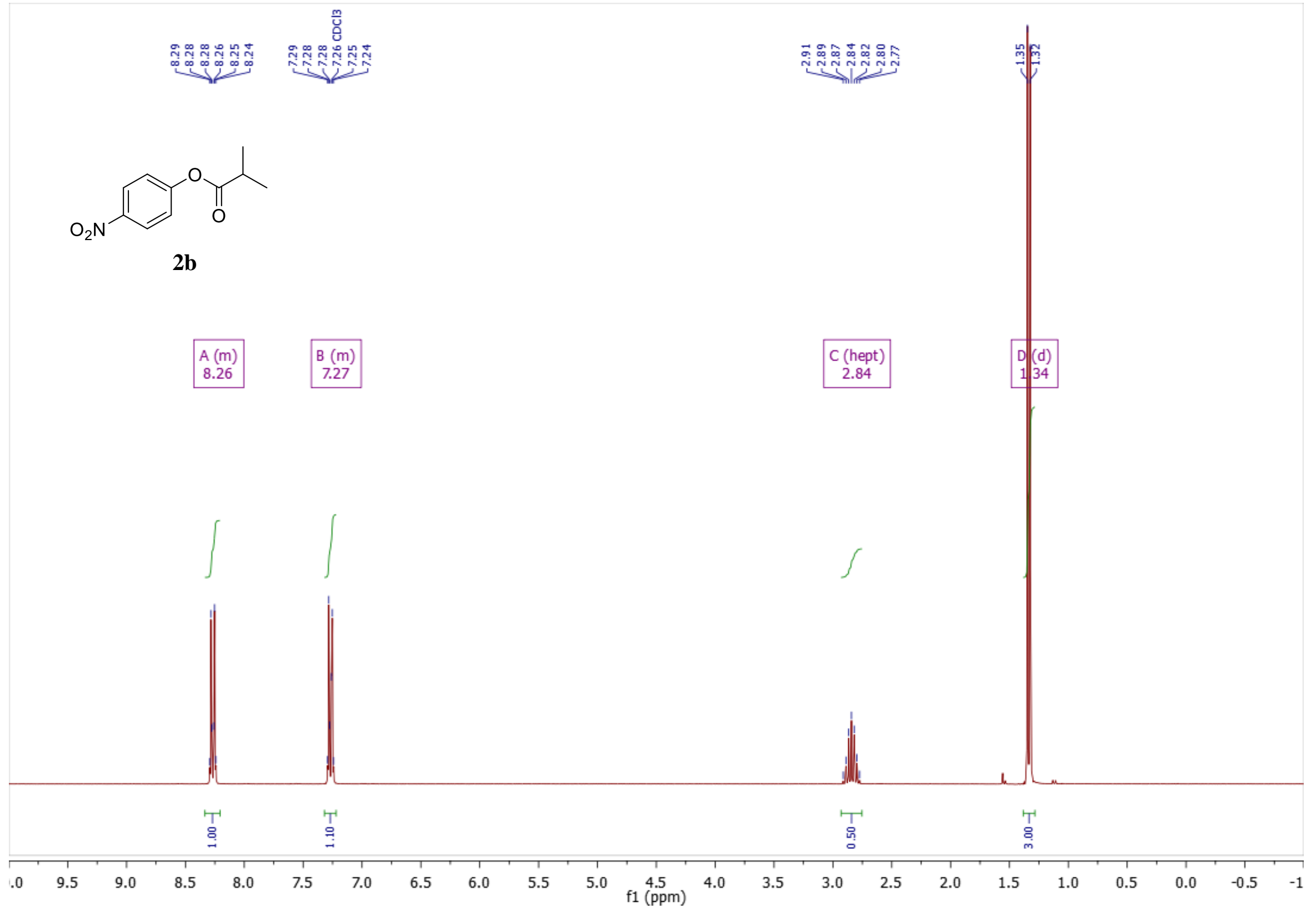


${ }^{1} \mathrm{H}$ NMR (300 MHz, $\left.\mathrm{CDCl}_{3}, \mathrm{ppm}\right)$

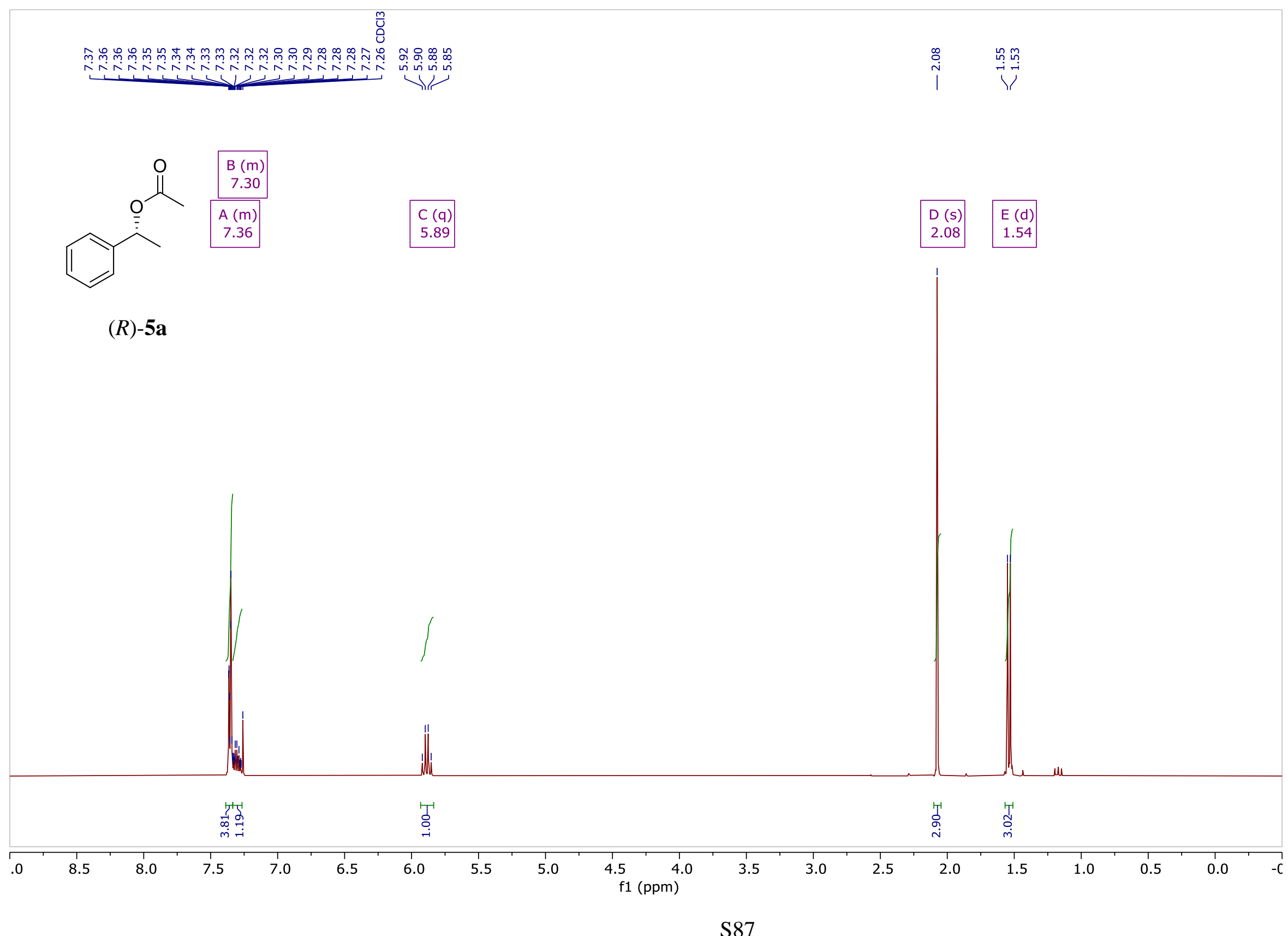


${ }^{1} \mathrm{H}$ NMR (300 MHz, $\left.\mathrm{CDCl}_{3}, \mathrm{ppm}\right)$

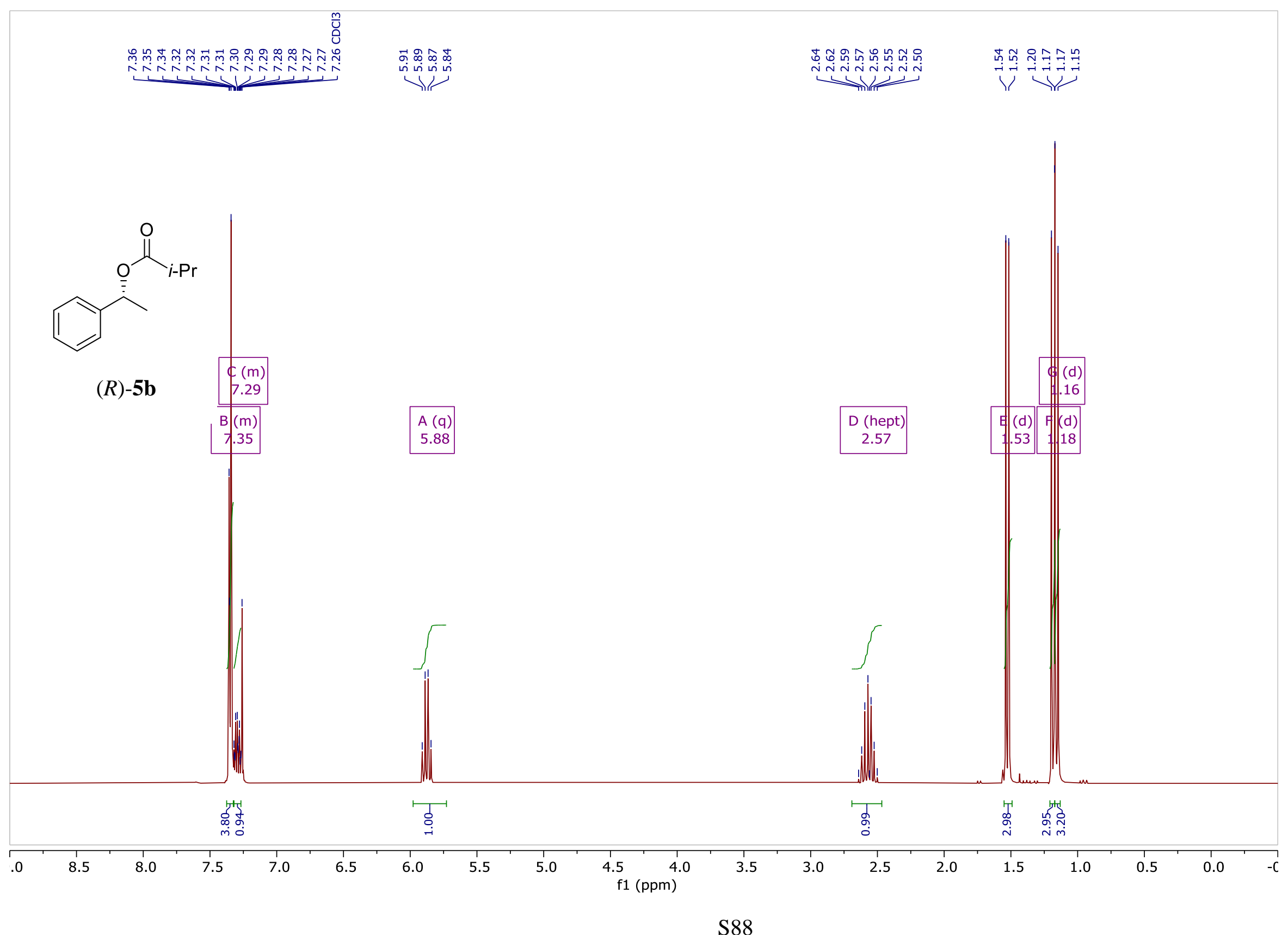


${ }^{1} \mathrm{H}$ NMR (300 MHz, $\left.\mathrm{CDCl}_{3}, \mathrm{ppm}\right)$




${ }^{1} \mathrm{H}$ NMR (300 MHz, $\left.\mathrm{CDCl}_{3}, \mathrm{ppm}\right)$

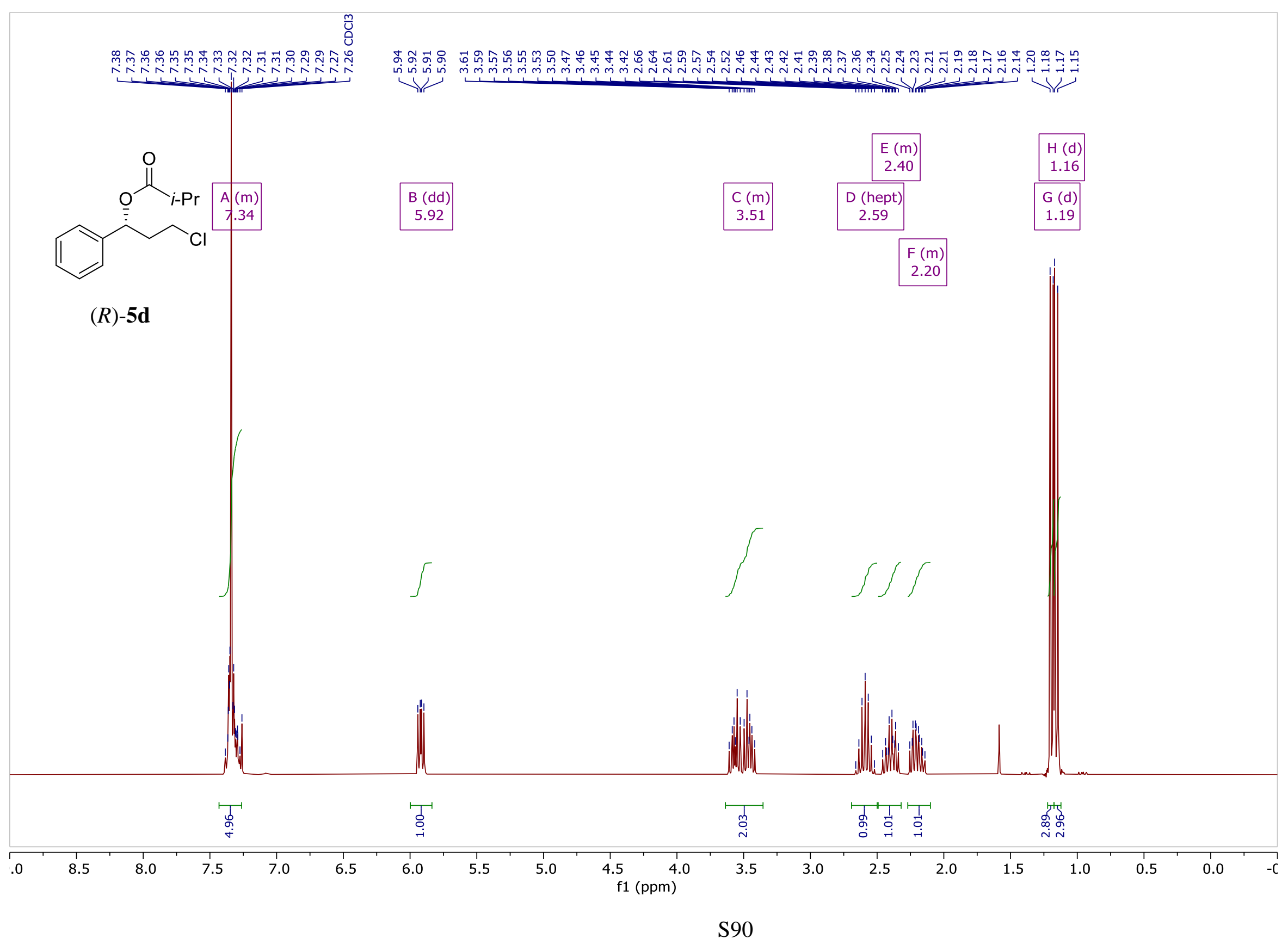


${ }^{13} \mathrm{C}\left\{{ }^{1} \mathrm{H}\right\}$ NMR $\left(75 \mathrm{MHz}, \mathrm{CDCl}_{3}, \mathrm{ppm}\right)$
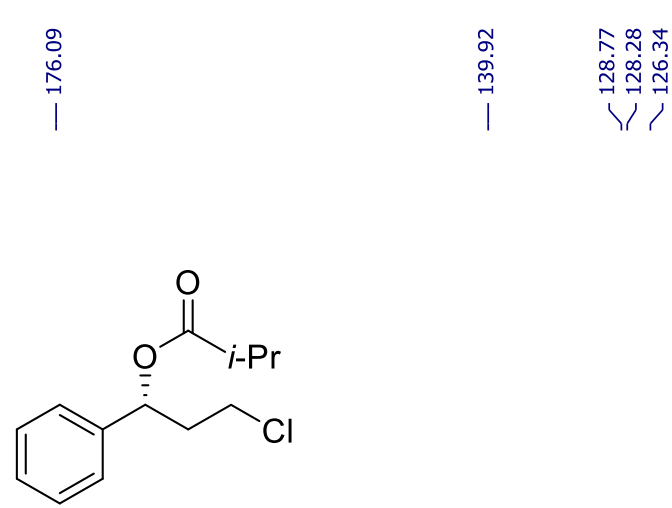

(R)-5d

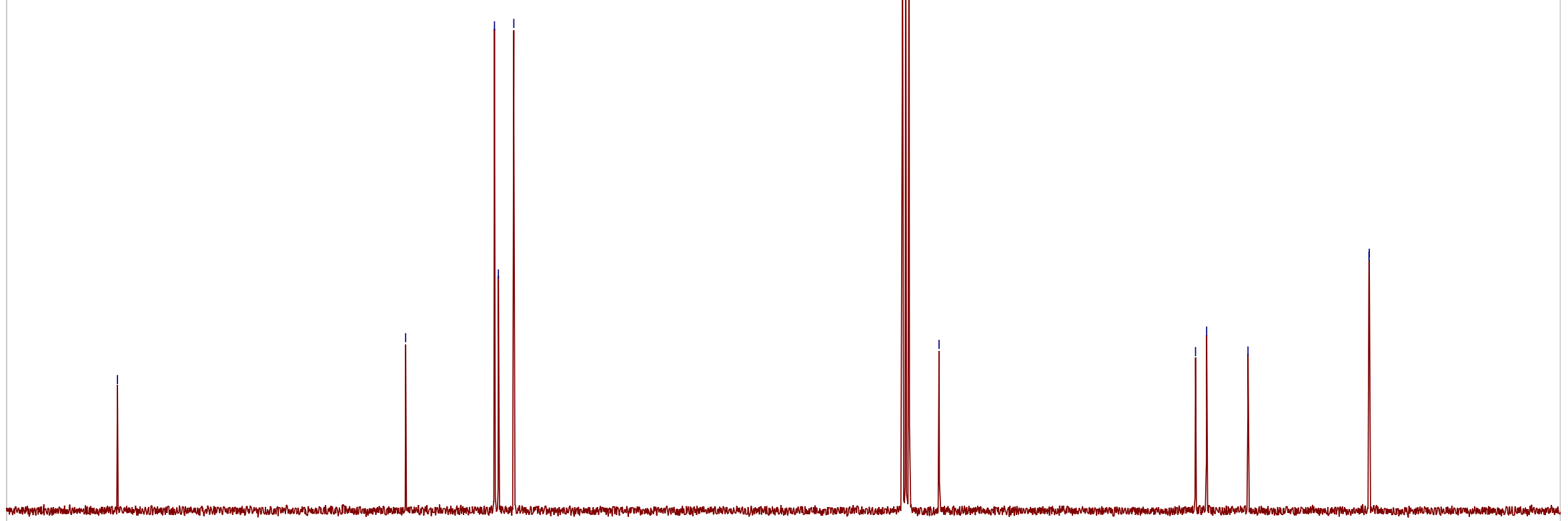


${ }^{1} \mathrm{H}$ NMR (300 MHz, $\left.\mathrm{CDCl}_{3}, \mathrm{ppm}\right)$

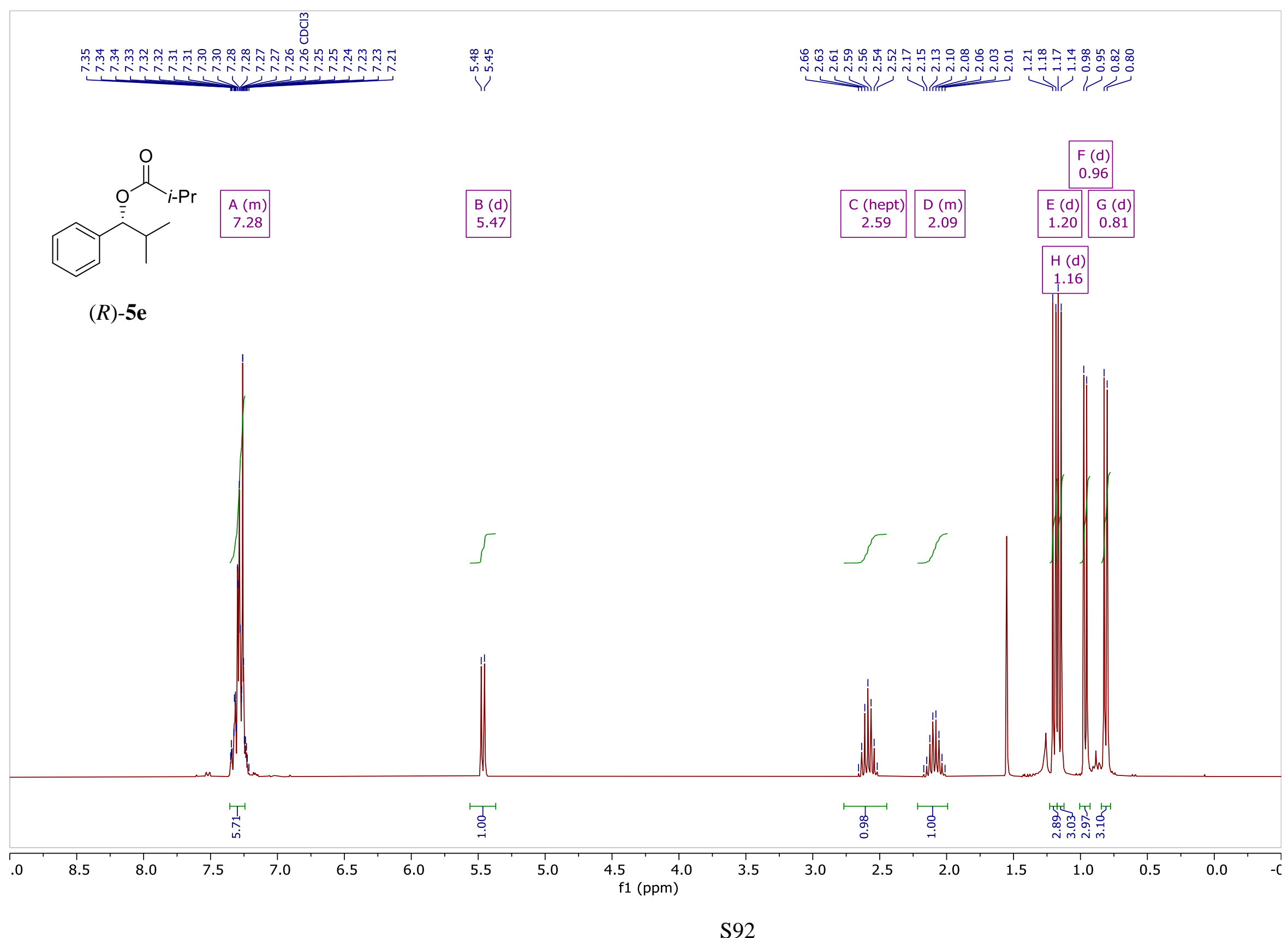


${ }^{1} \mathrm{H}$ NMR (300 MHz, $\left.\mathrm{CDCl}_{3}, \mathrm{ppm}\right)$

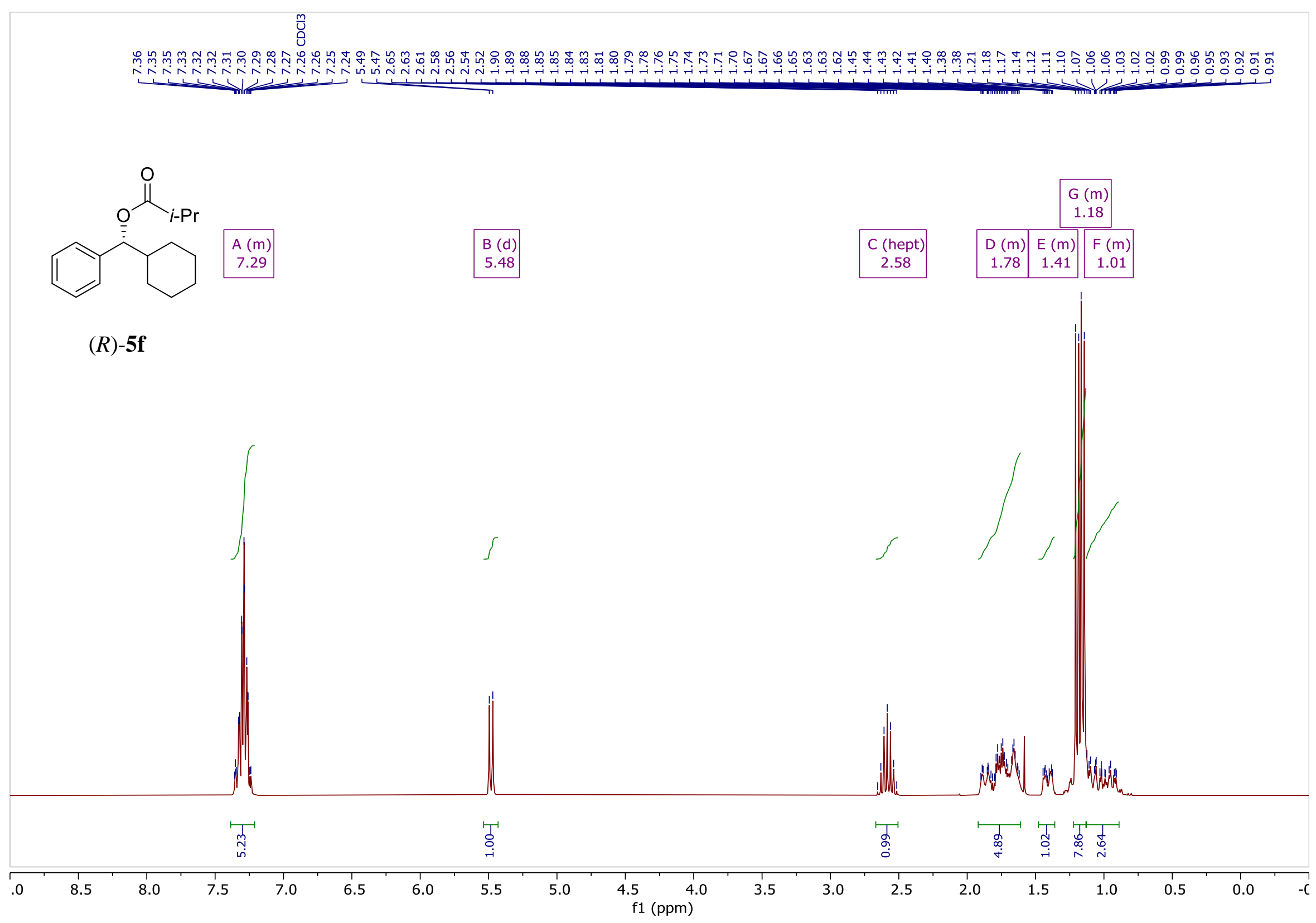


${ }^{13} \mathrm{C}\left\{{ }^{1} \mathrm{H}\right\}$ NMR $\left(75 \mathrm{MHz}, \mathrm{CDCl}_{3}, \mathrm{ppm}\right)$

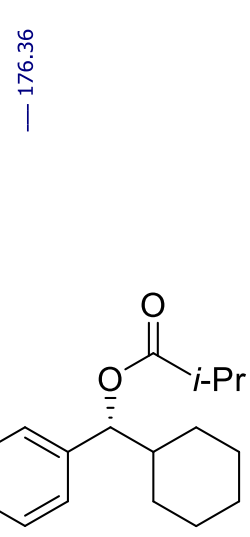

|

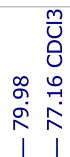

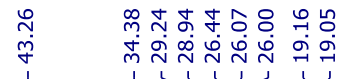

(R)-5f

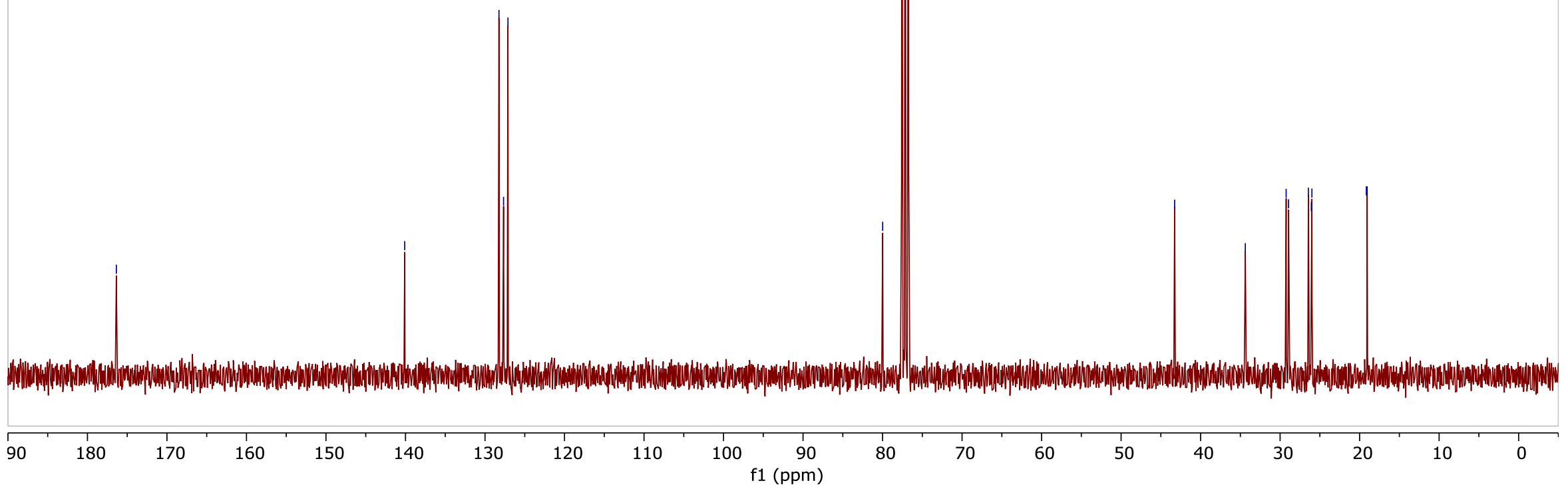


${ }^{1} \mathrm{H}$ NMR (300 MHz, $\left.\mathrm{CDCl}_{3}, \mathrm{ppm}\right)$

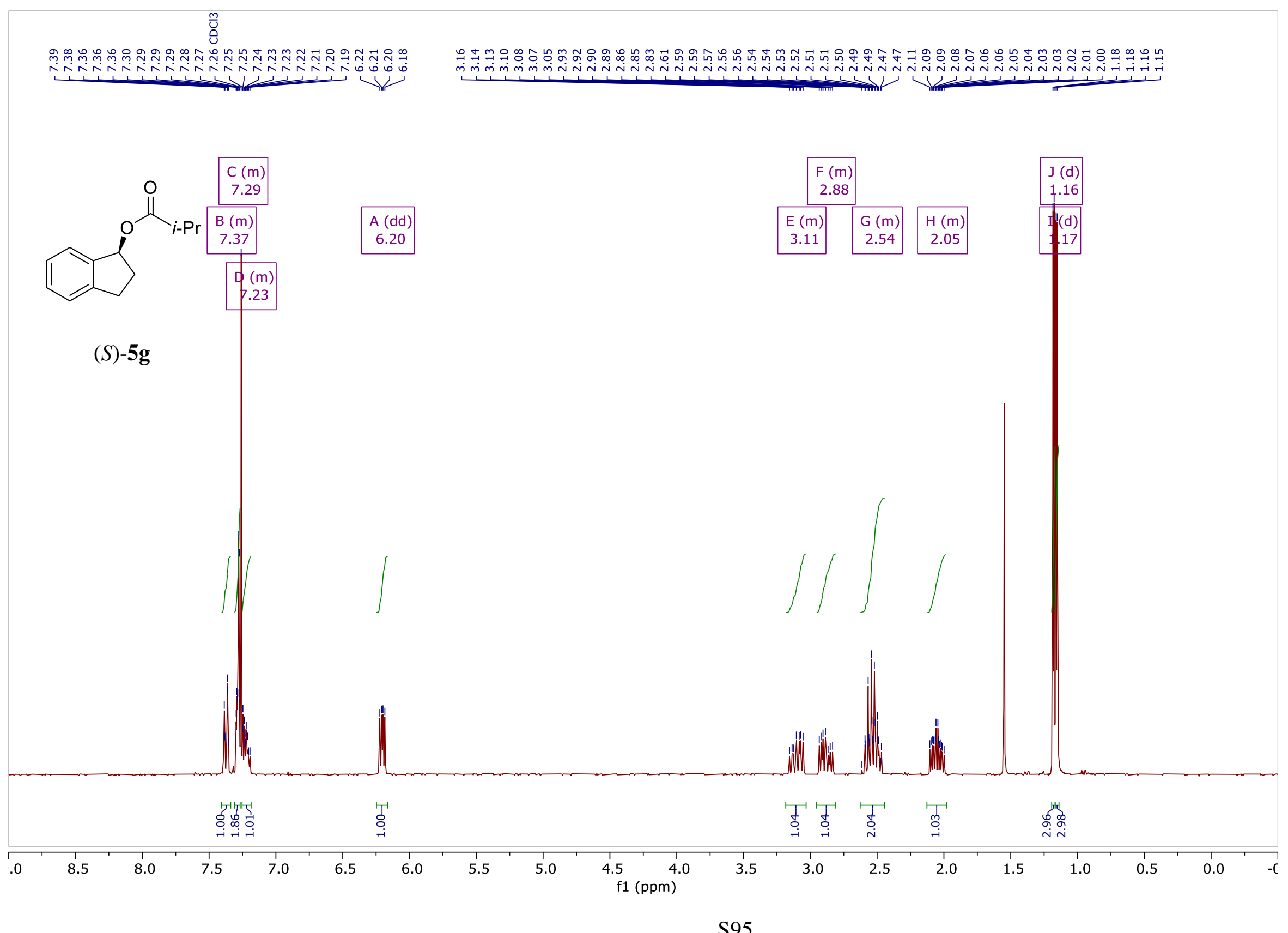


${ }^{1} \mathrm{H}$ NMR (300 MHz, $\left.\mathrm{CDCl}_{3}, \mathrm{ppm}\right)$

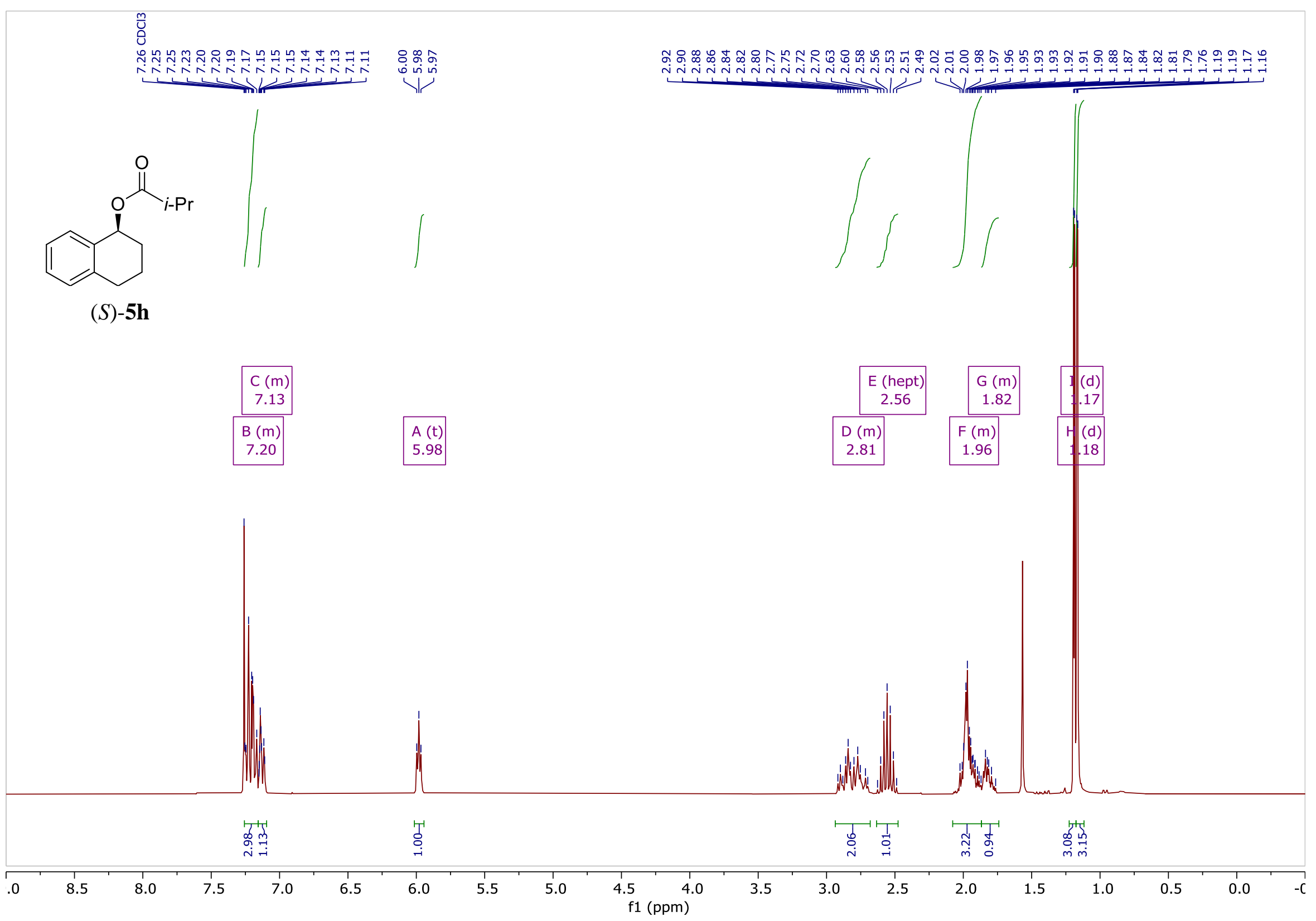


${ }^{1} \mathrm{H}$ NMR (300 MHz, $\left.\mathrm{CDCl}_{3}, \mathrm{ppm}\right)$

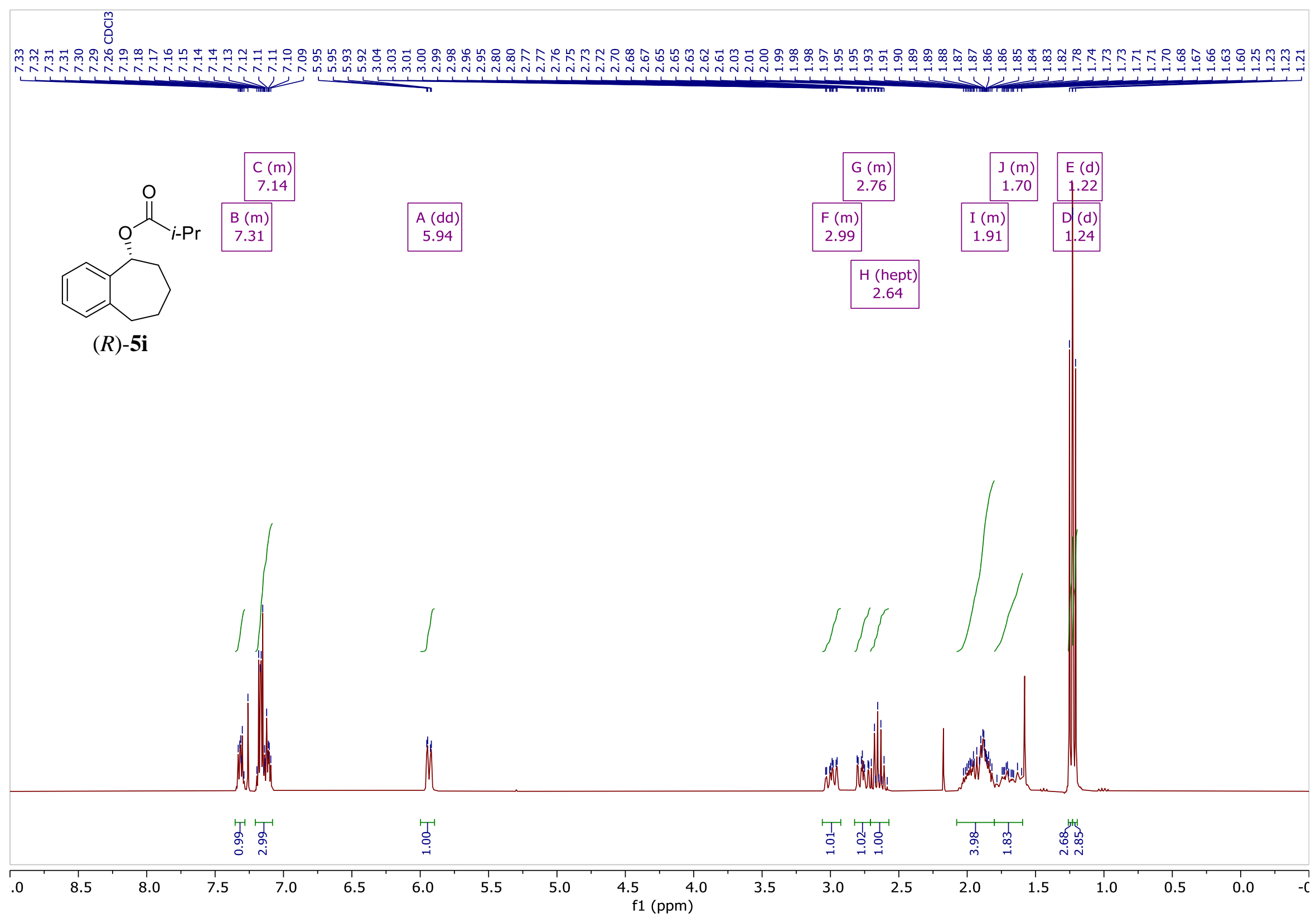


${ }^{13} \mathrm{C}\left\{{ }^{1} \mathrm{H}\right\} \operatorname{NMR}\left(75 \mathrm{MHz}, \mathrm{CDCl}_{3}, \mathrm{ppm}\right)$
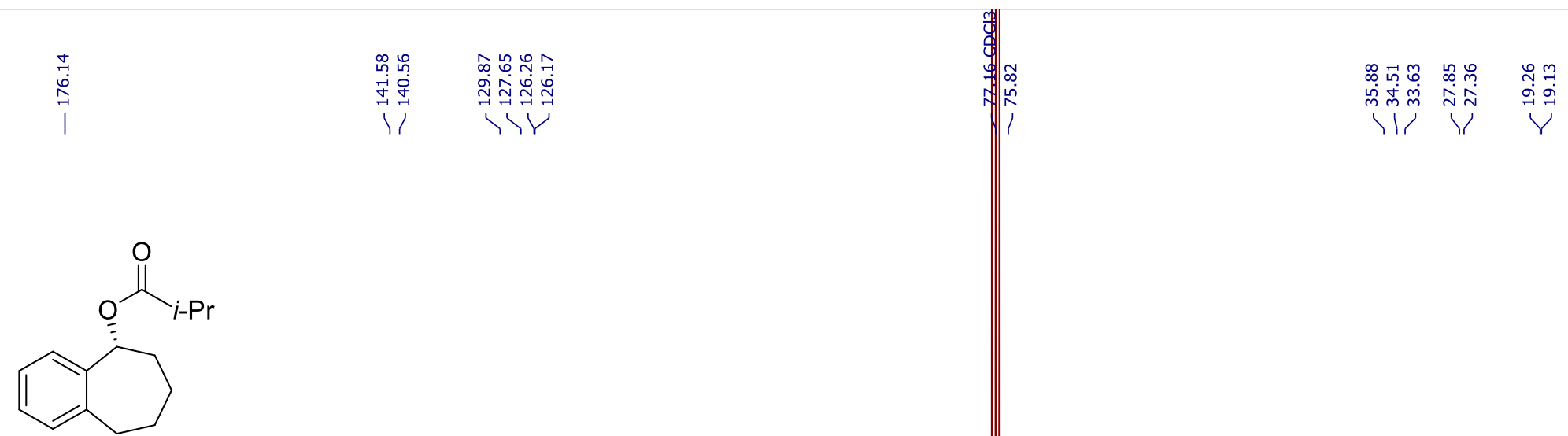

$(R)-5 \mathbf{i}$

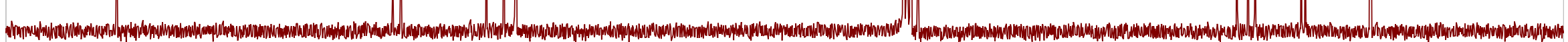


${ }^{1} \mathrm{H}$ NMR (300 MHz, $\left.\mathrm{CDCl}_{3}, \mathrm{ppm}\right)$

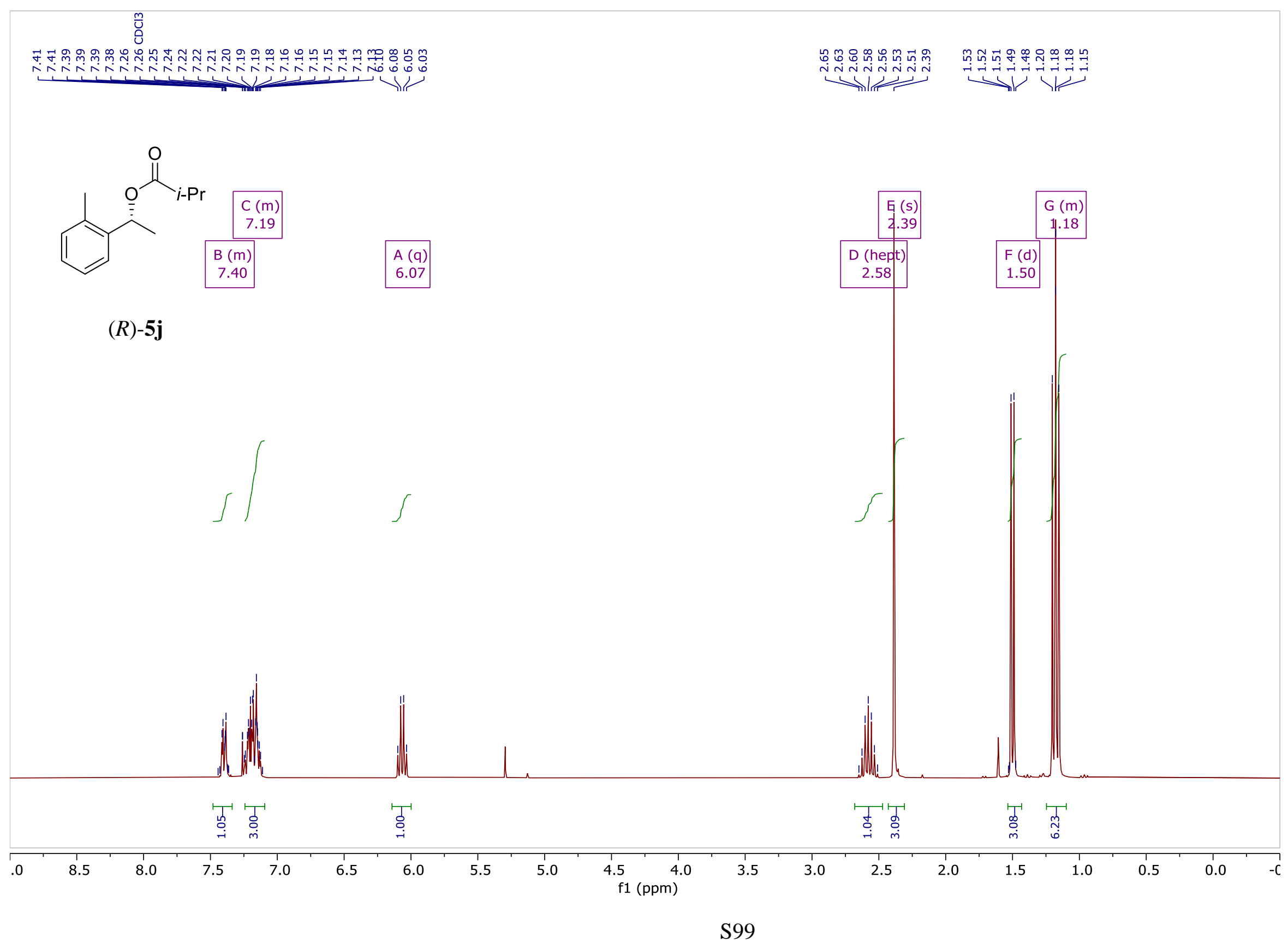


${ }^{13} \mathrm{C}\left\{{ }^{1} \mathrm{H}\right\}$ NMR $\left(75 \mathrm{MHz}, \mathrm{CDCl}_{3}, \mathrm{ppm}\right)$

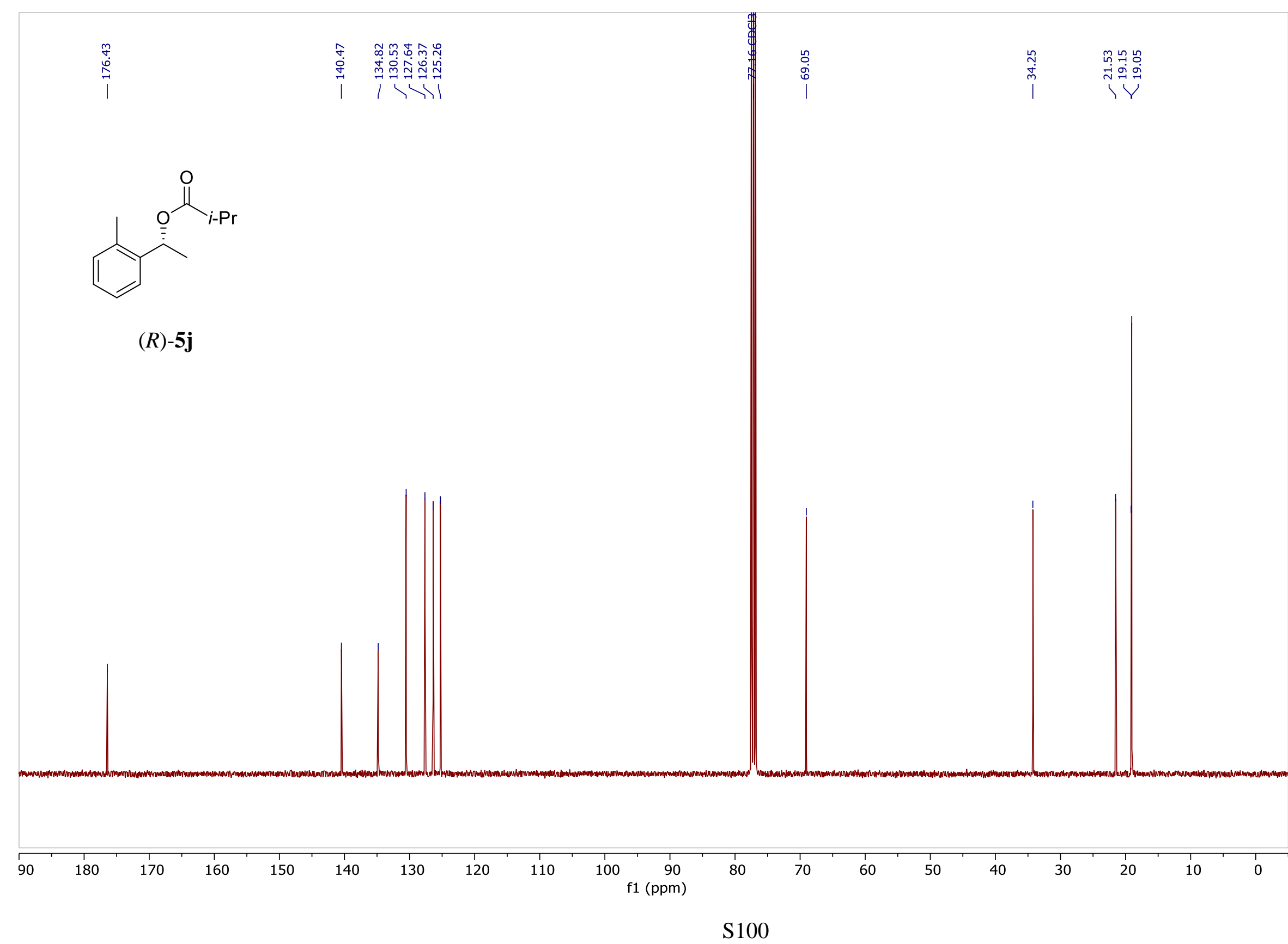


${ }^{1} \mathrm{H}$ NMR (300 MHz, $\left.\mathrm{CDCl}_{3}, \mathrm{ppm}\right)$

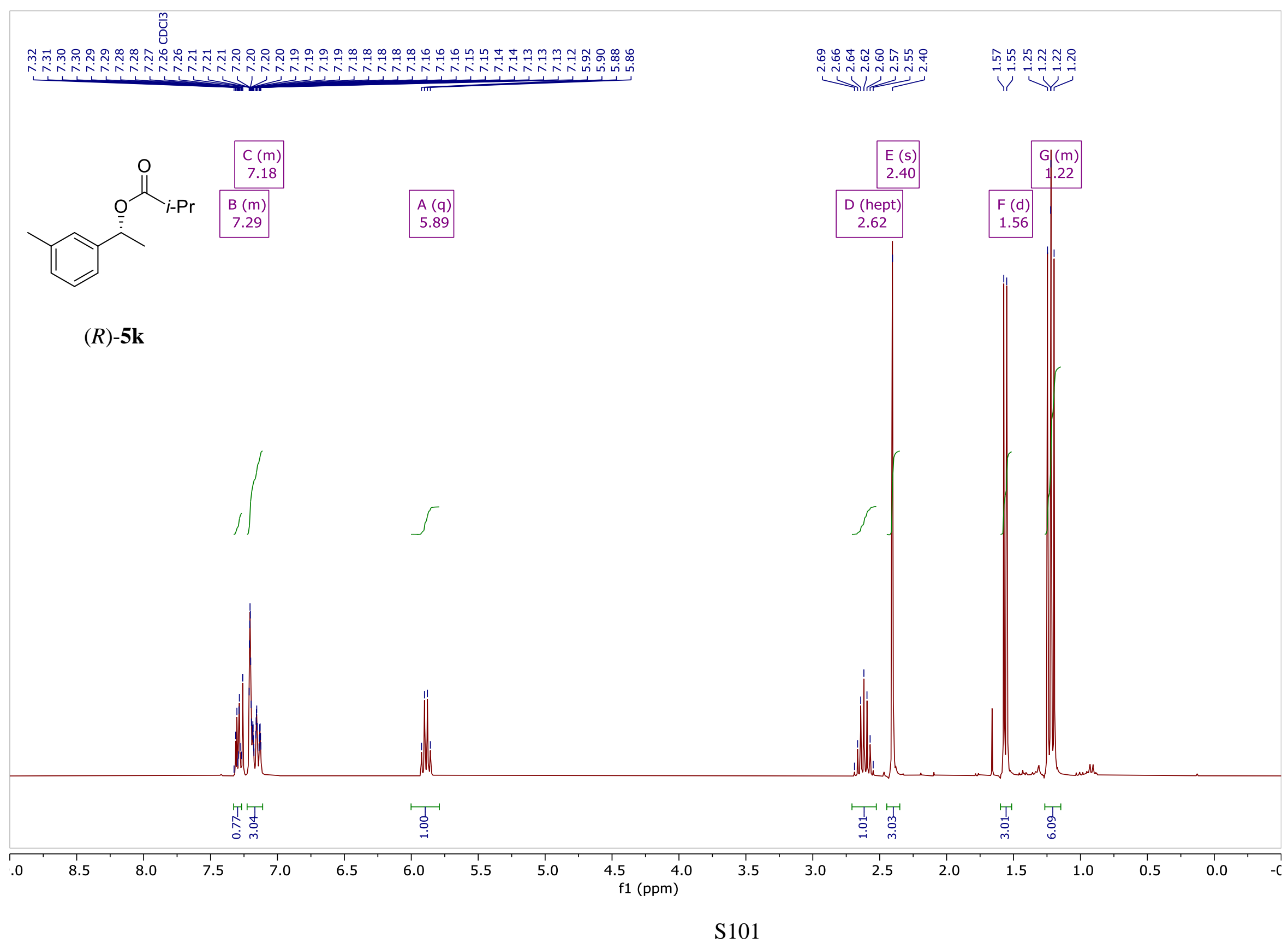


${ }^{13} \mathrm{C}\left\{{ }^{1} \mathrm{H}\right\}$ NMR $\left(75 \mathrm{MHz}, \mathrm{CDCl}_{3}, \mathrm{ppm}\right)$

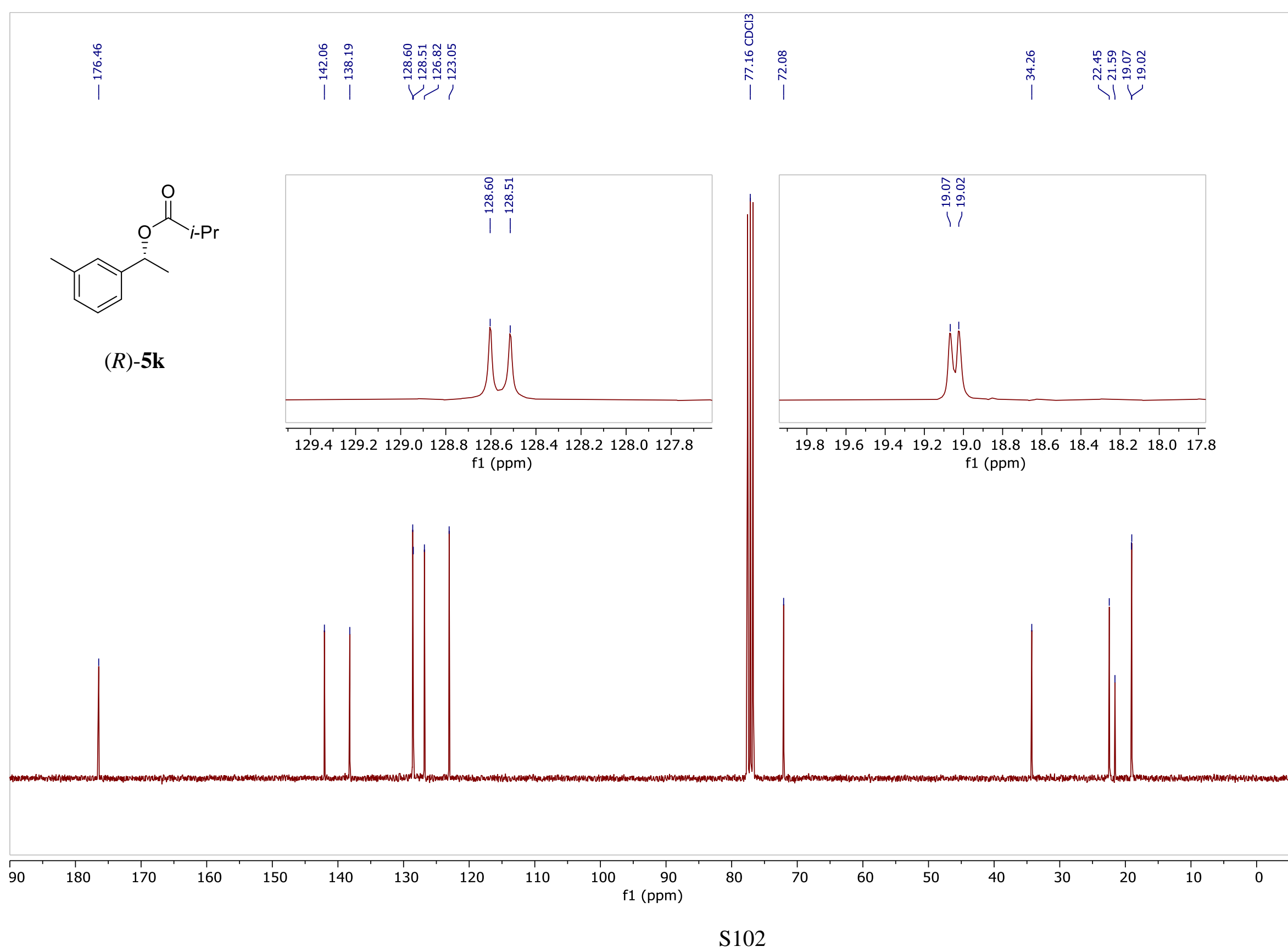


${ }^{1} \mathrm{H}$ NMR (300 MHz, $\left.\mathrm{CDCl}_{3}, \mathrm{ppm}\right)$

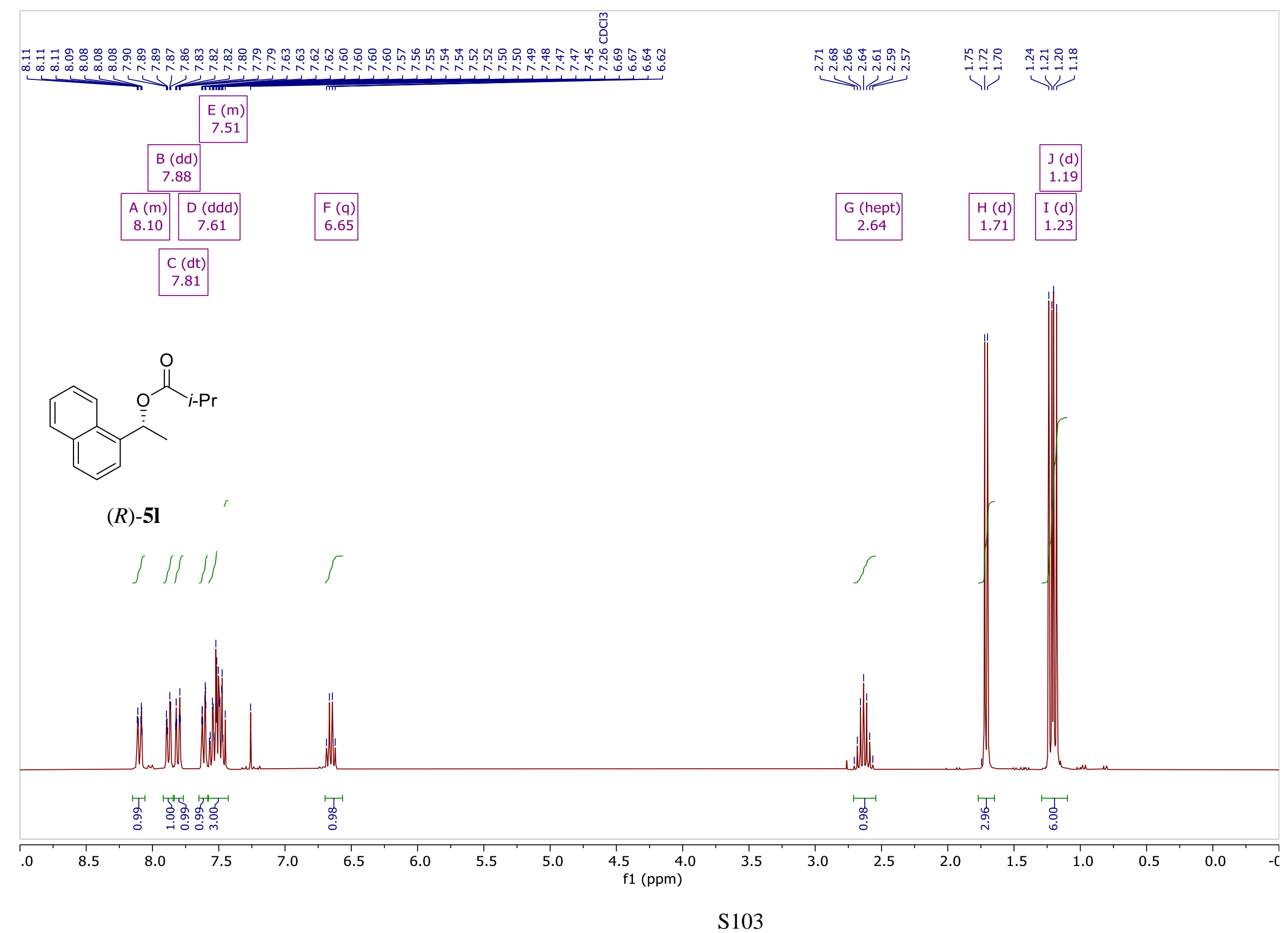


${ }^{1} \mathrm{H}$ NMR (300 MHz, $\left.\mathrm{CDCl}_{3}, \mathrm{ppm}\right)$

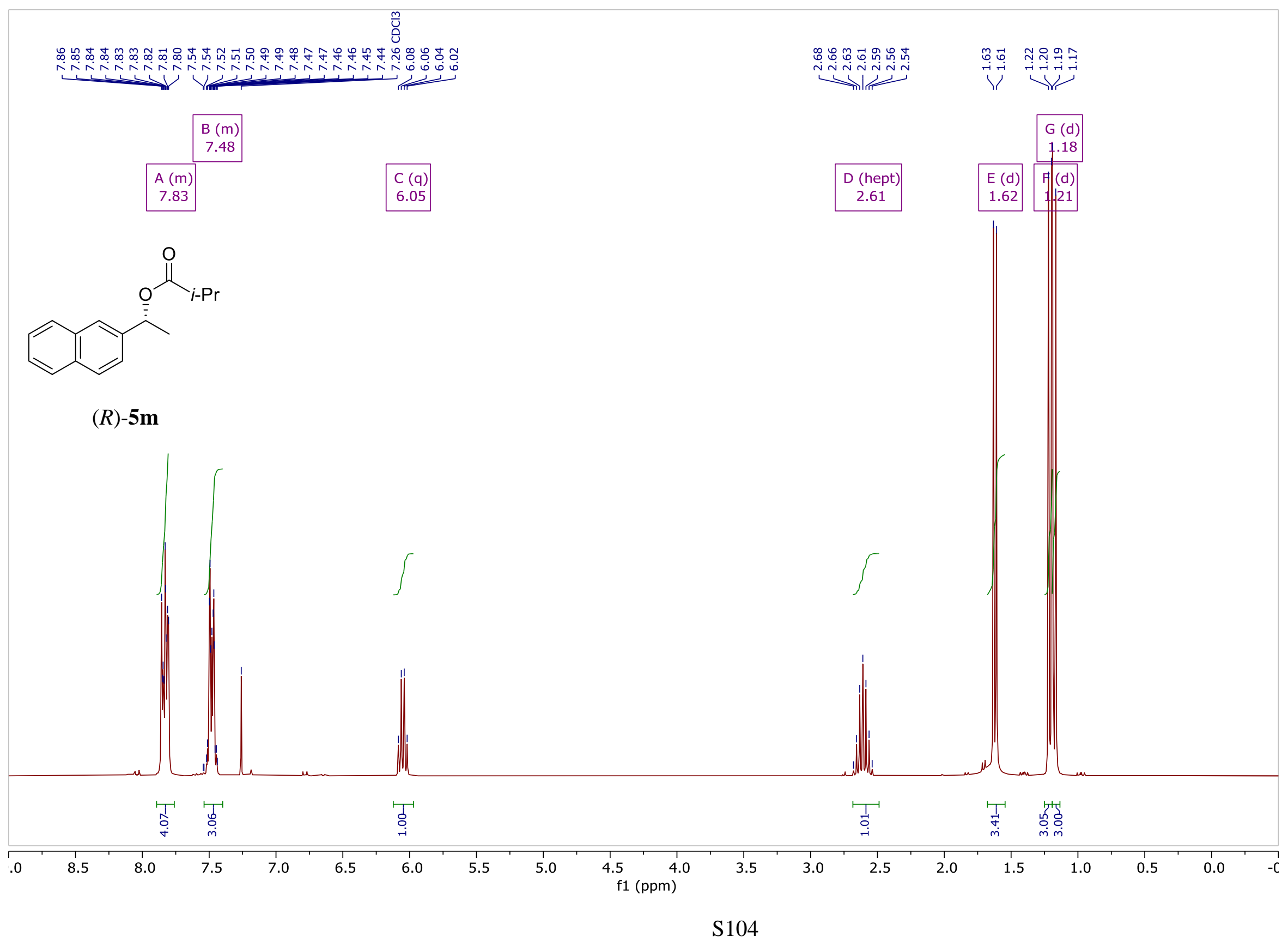


${ }^{1} \mathrm{H}$ NMR (300 MHz, $\left.\mathrm{CDCl}_{3}, \mathrm{ppm}\right)$




${ }^{13} \mathrm{C}\left\{{ }^{1} \mathrm{H}\right\}$ NMR $\left(75 \mathrm{MHz}, \mathrm{CDCl}_{3}, \mathrm{ppm}\right)$





$(R)-5 n$
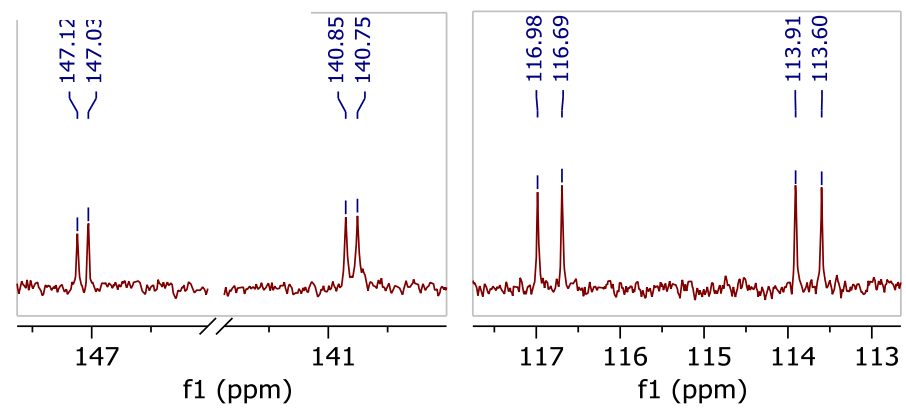

f1 (ppm)
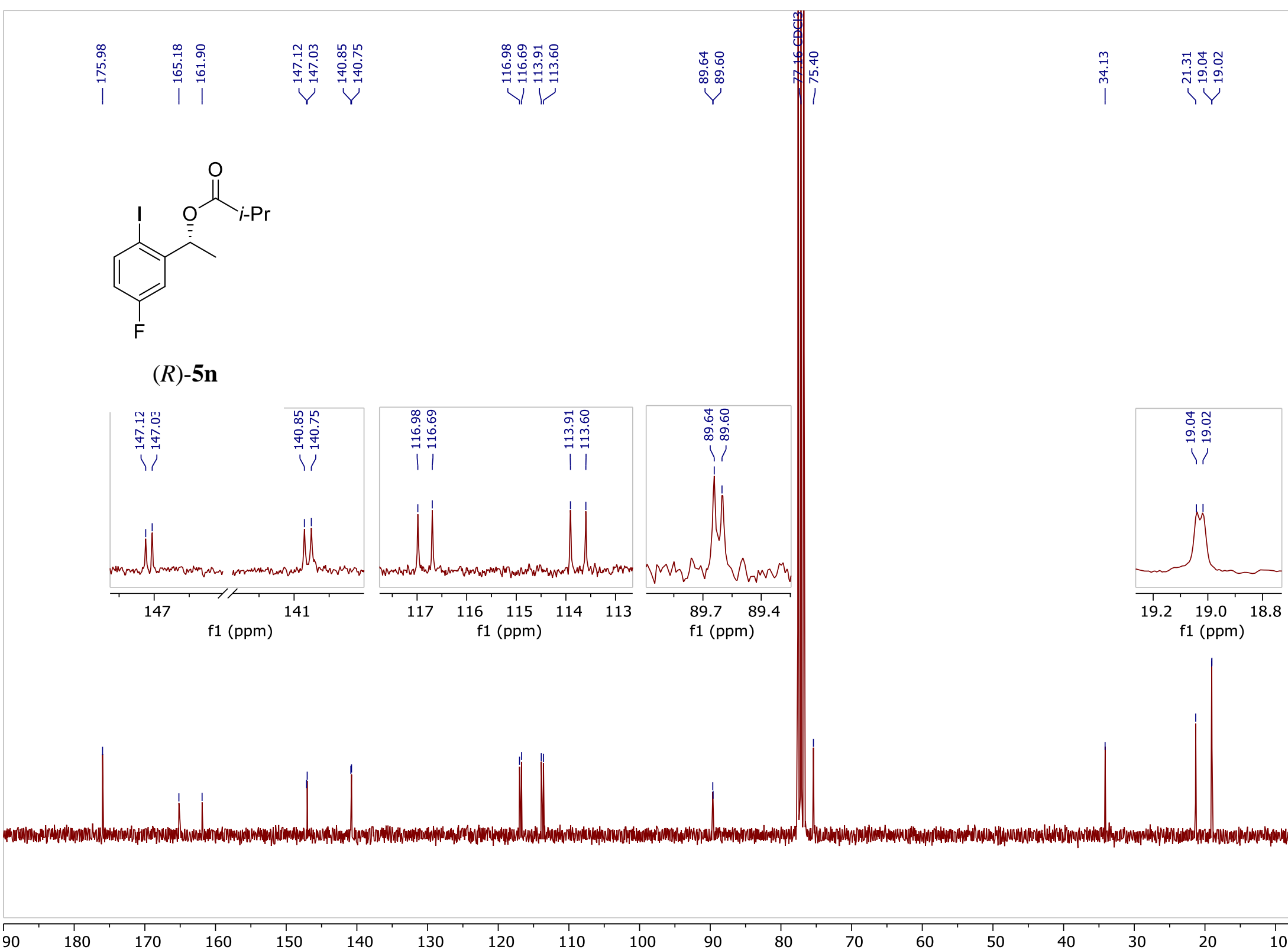

100

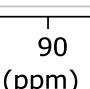

1 (ppm)
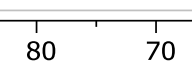

60

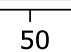

40


10 
${ }^{19} \mathrm{~F} \mathrm{NMR}\left(376 \mathrm{MHz}, \mathrm{CDCl}_{3}, \mathrm{ppm}\right)$

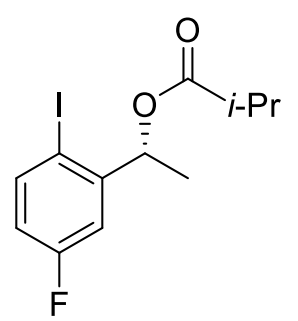

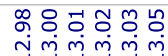

ن

$(R)-5 n$

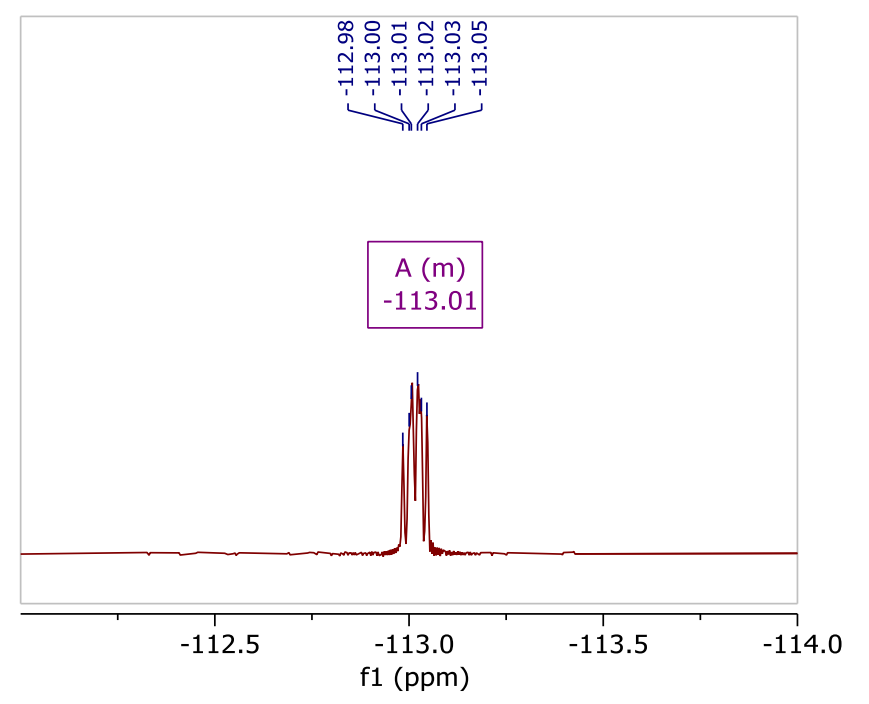


${ }^{1} \mathrm{H}$ NMR (300 MHz, $\left.\mathrm{CDCl}_{3}, \mathrm{ppm}\right)$

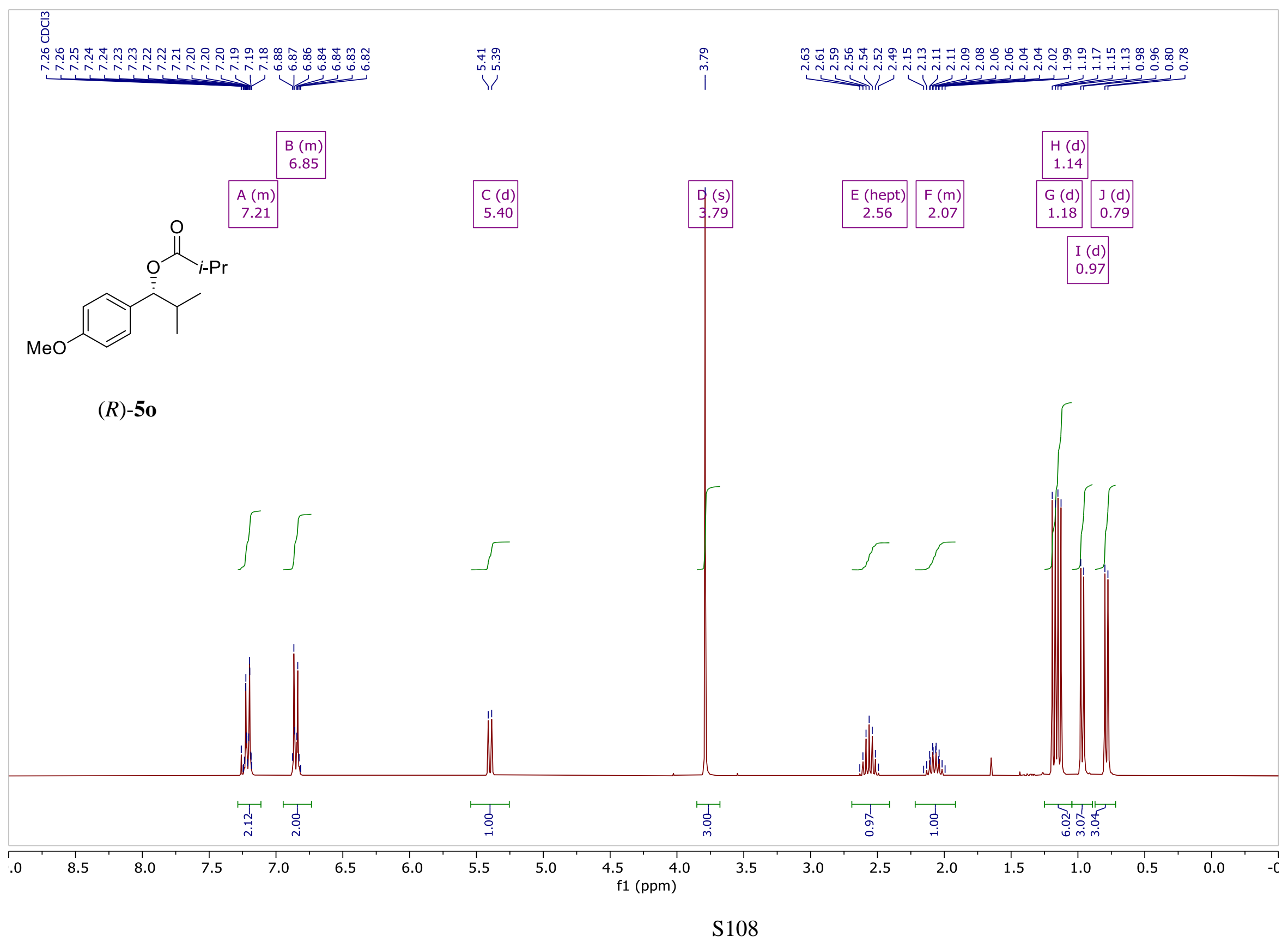


${ }^{13} \mathrm{C}\left\{{ }^{1} \mathrm{H}\right\}$ NMR $\left(75 \mathrm{MHz}, \mathrm{CDCl}_{3}, \mathrm{ppm}\right)$

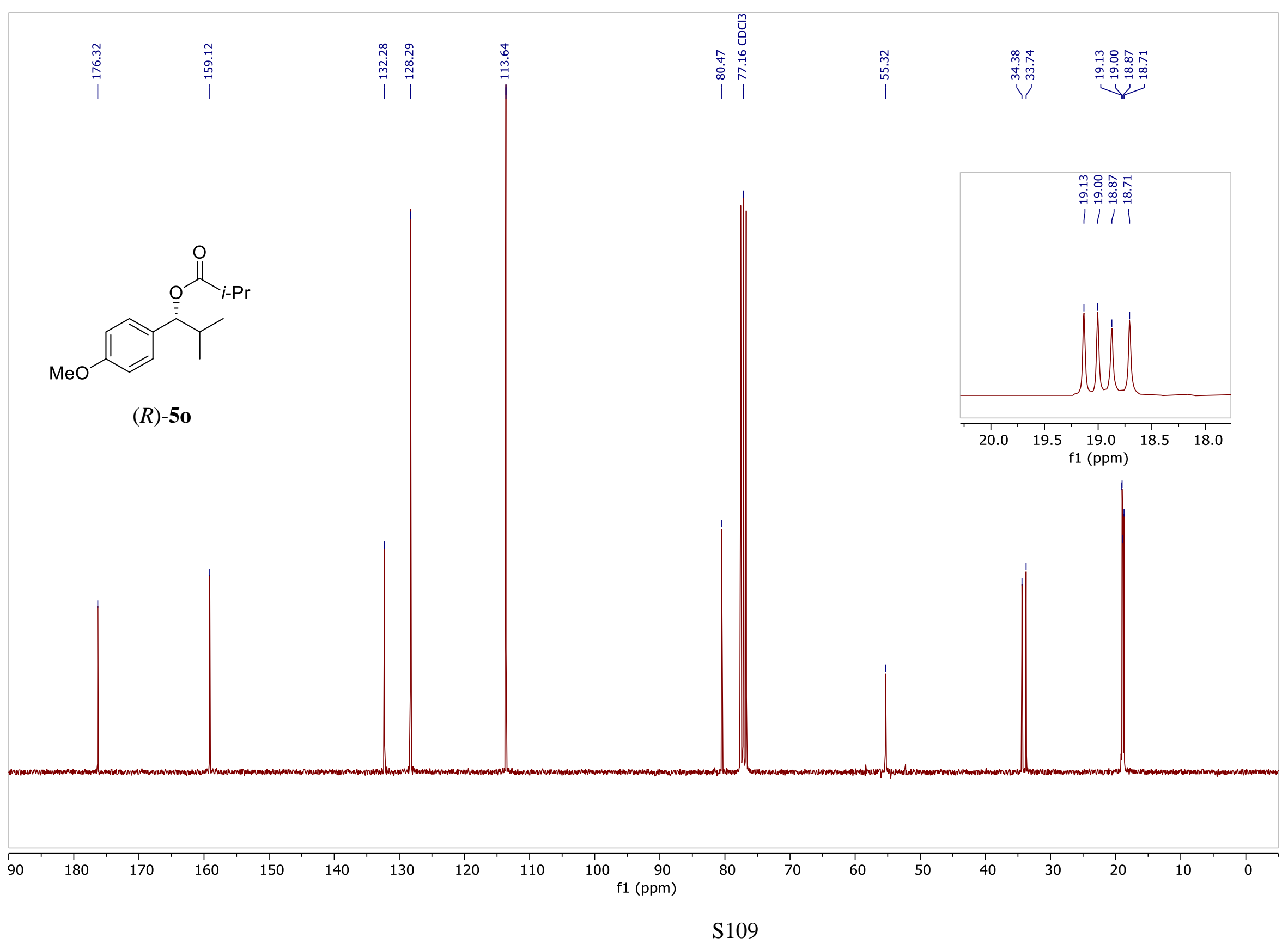


${ }^{1} \mathrm{H}$ NMR (300 MHz, $\left.\mathrm{CDCl}_{3}, \mathrm{ppm}\right)$

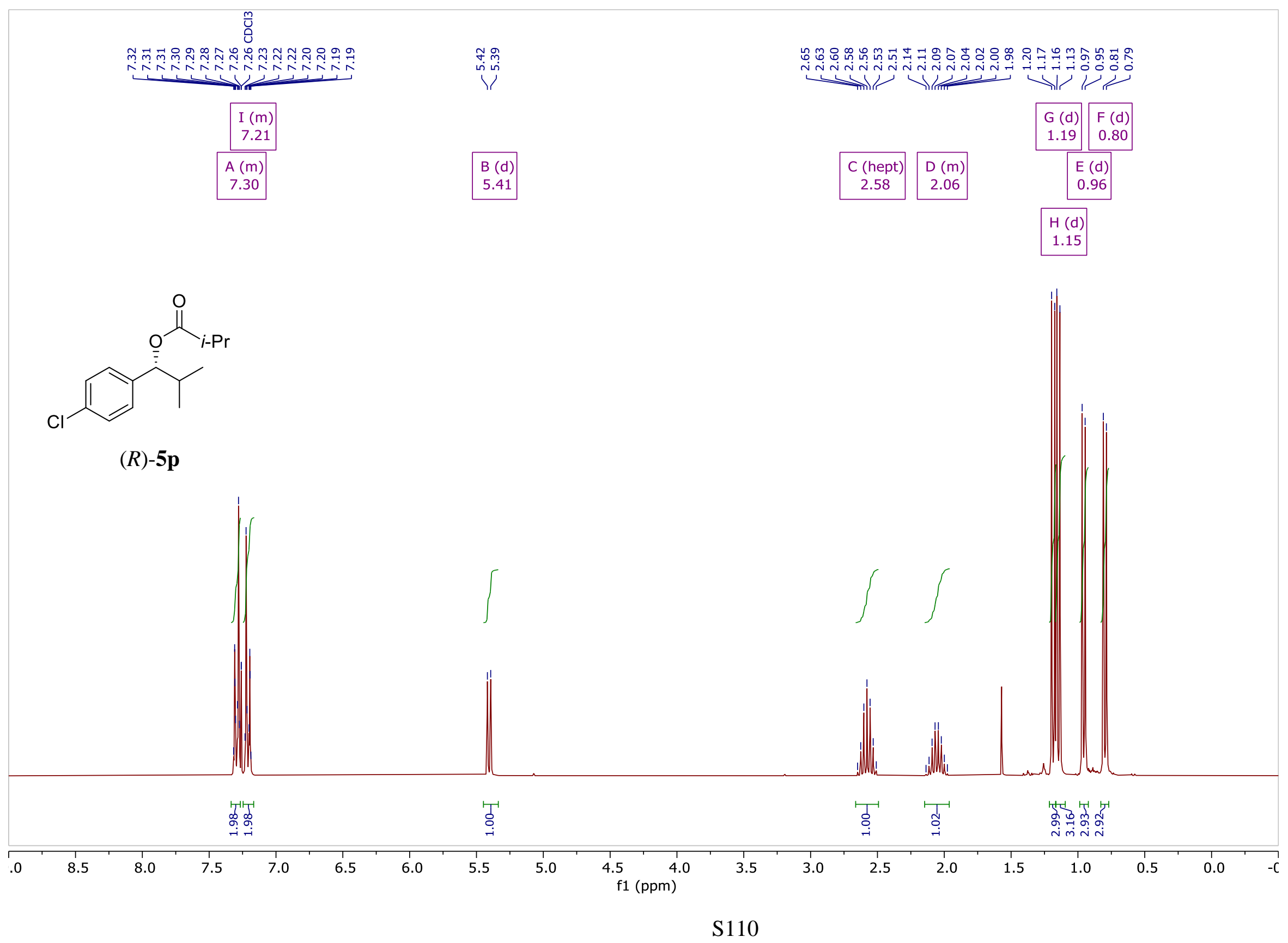


${ }^{13} \mathrm{C}\left\{{ }^{1} \mathrm{H}\right\}$ NMR $\left(75 \mathrm{MHz}, \mathrm{CDCl}_{3}, \mathrm{ppm}\right)$

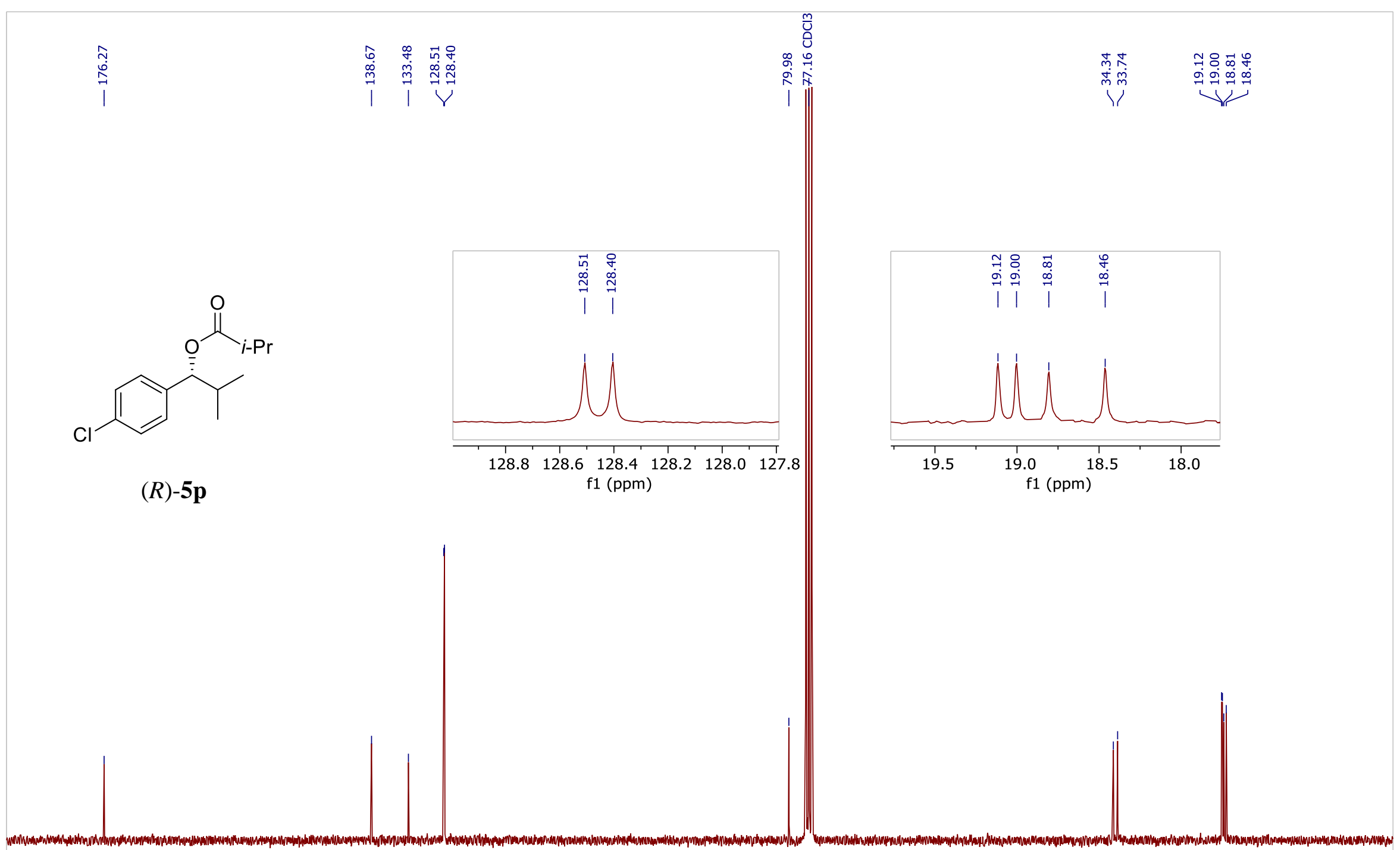


${ }^{1} \mathrm{H}$ NMR (300 MHz, $\left.\mathrm{CDCl}_{3}, \mathrm{ppm}\right)$

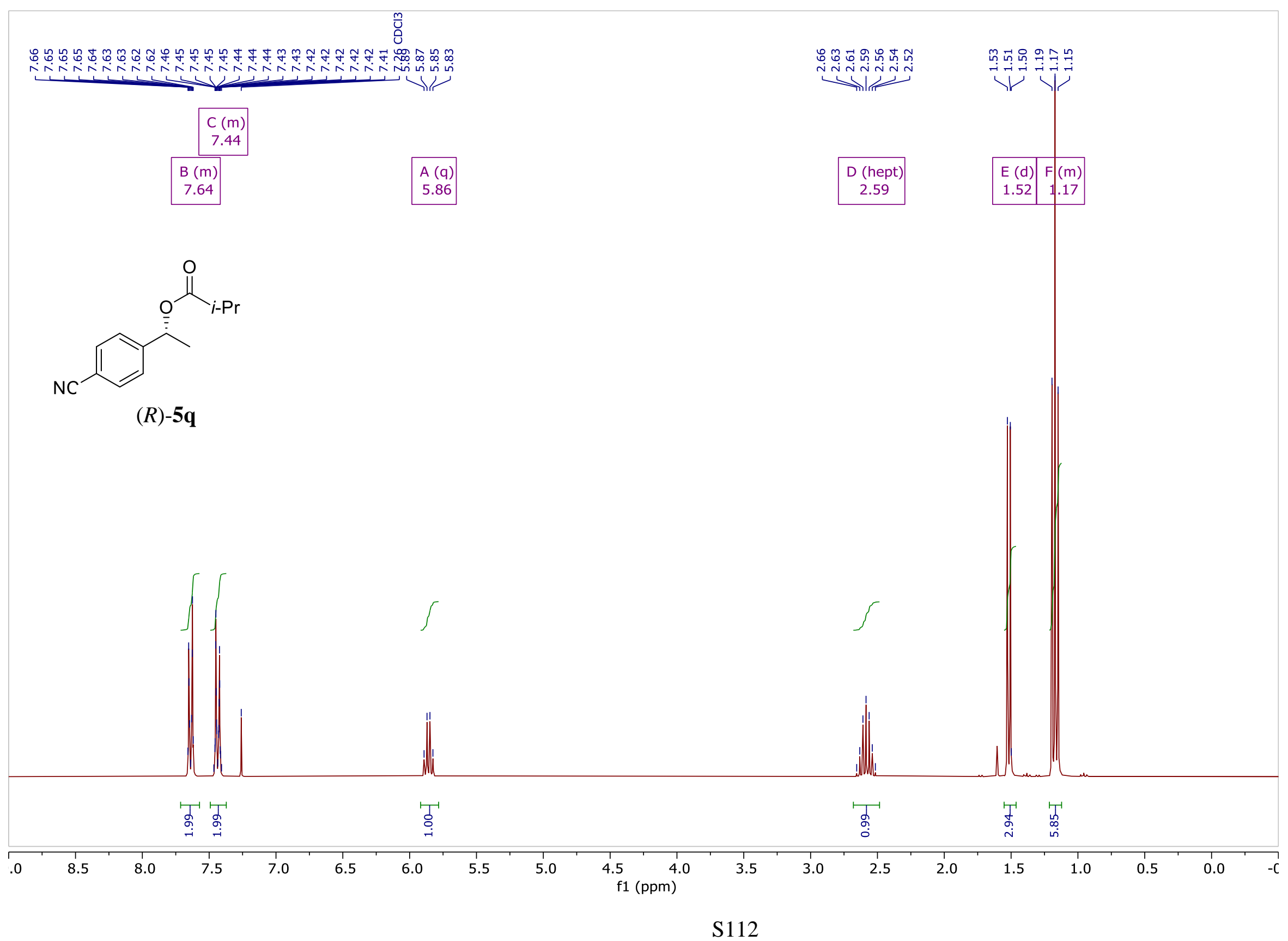


${ }^{13} \mathrm{C}\left\{{ }^{1} \mathrm{H}\right\}$ NMR $\left(75 \mathrm{MHz}, \mathrm{CDCl}_{3}, \mathrm{ppm}\right)$

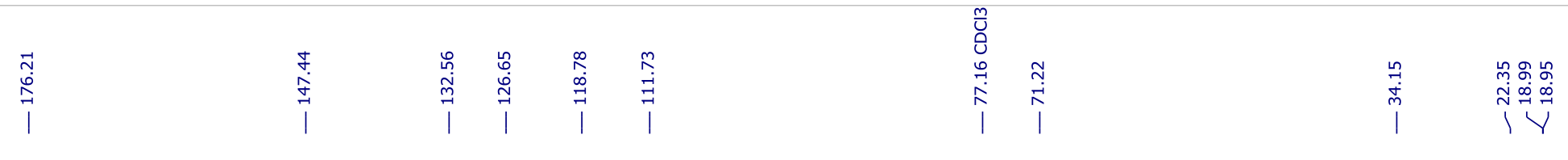

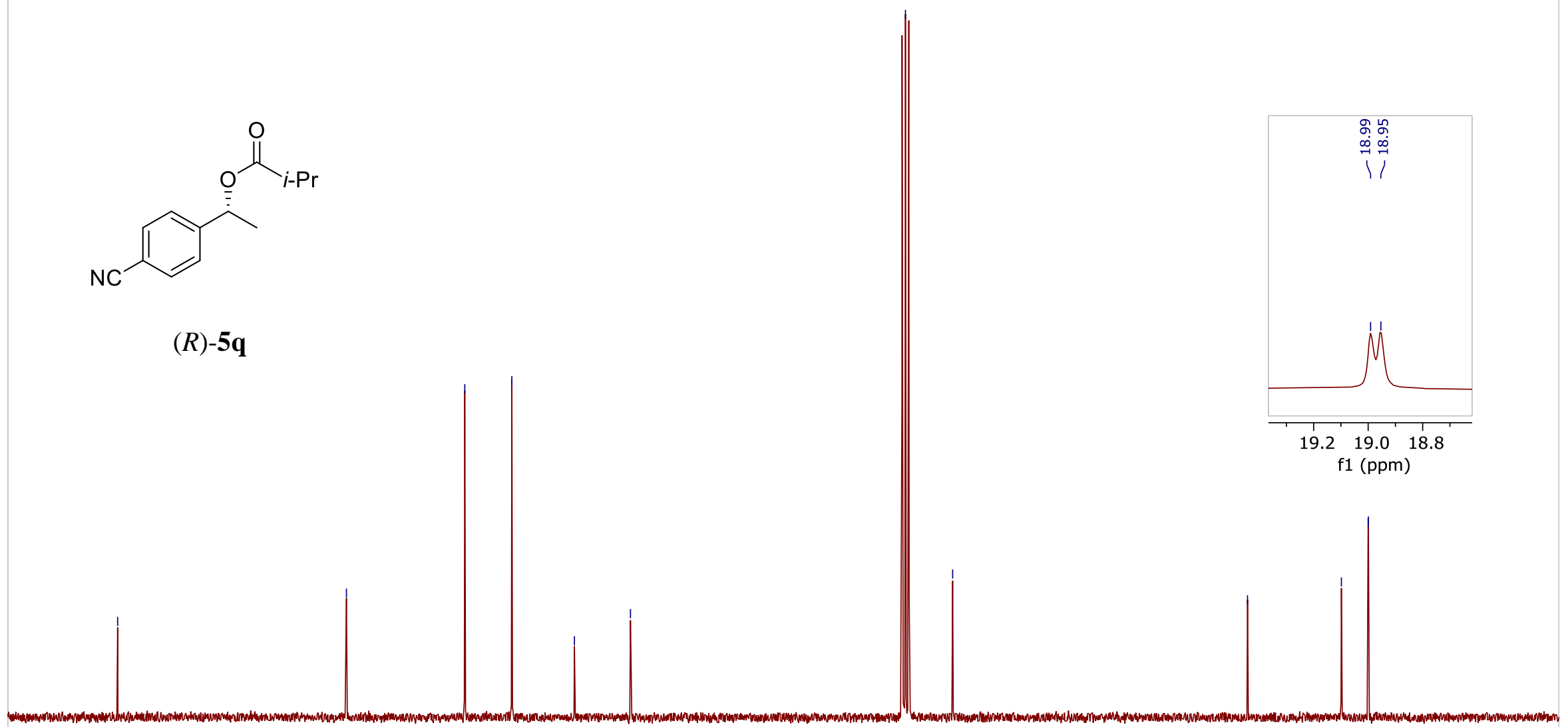


${ }^{1} \mathrm{H}$ NMR (300 MHz, $\left.\mathrm{CDCl}_{3}, \mathrm{ppm}\right)$

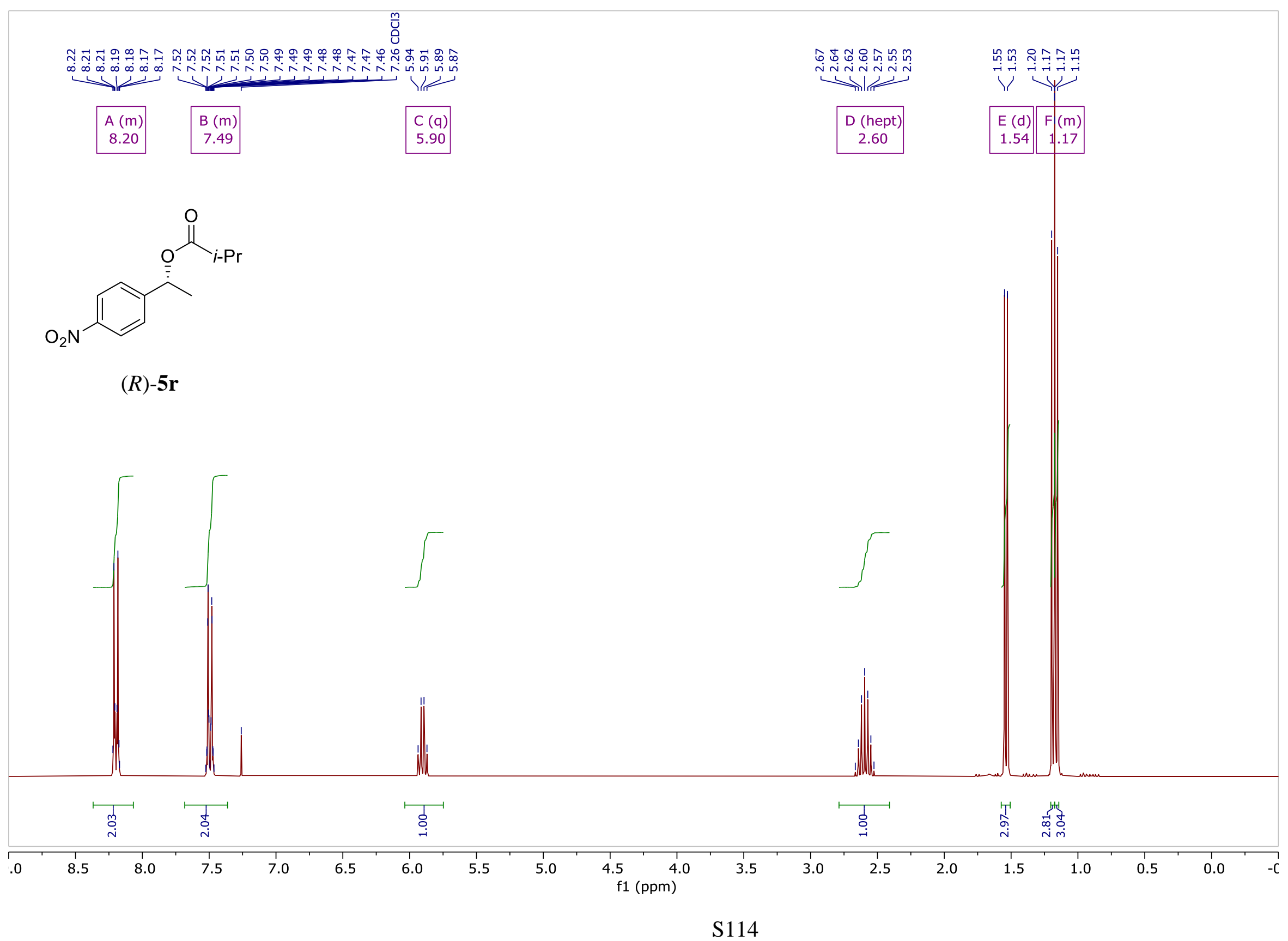


${ }^{13} \mathrm{C}\left\{{ }^{1} \mathrm{H}\right\}$ NMR $\left(75 \mathrm{MHz}, \mathrm{CDCl}_{3}, \mathrm{ppm}\right)$

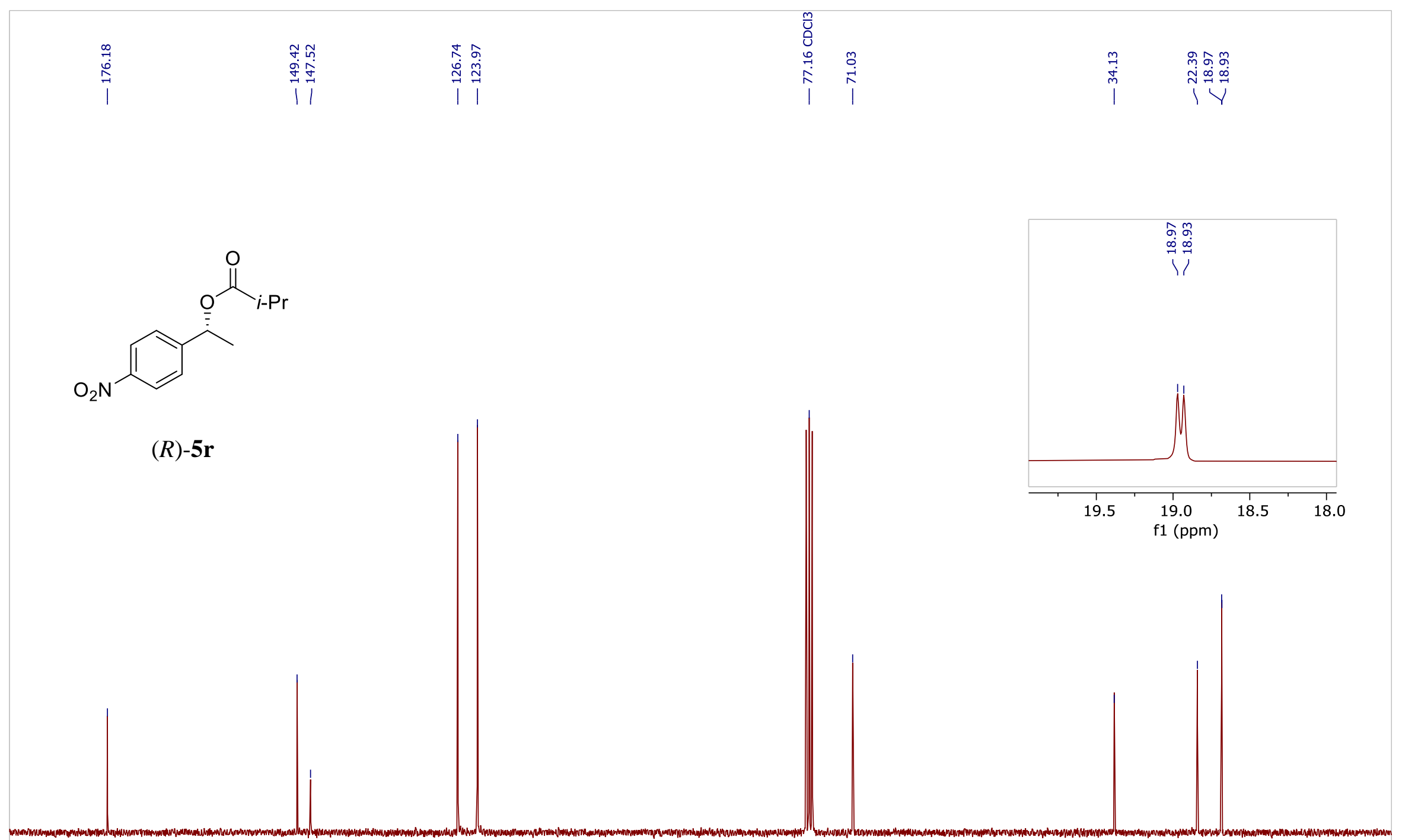

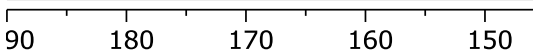
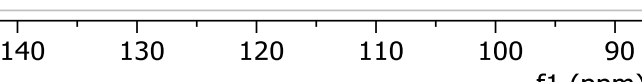

f1 (ppm) 
${ }^{1} \mathrm{H}$ NMR (300 MHz, $\left.\mathrm{CDCl}_{3}, \mathrm{ppm}\right)$

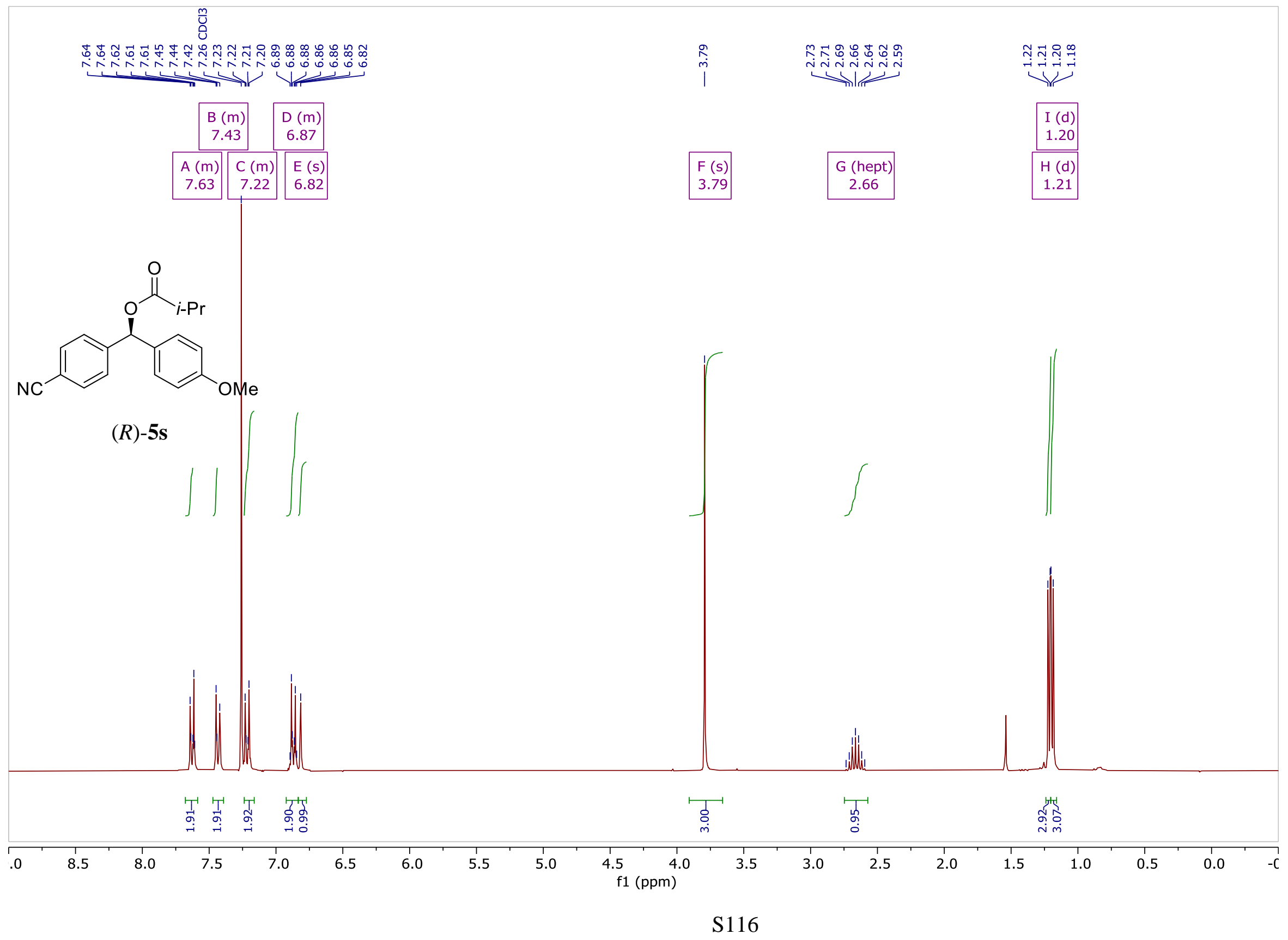


${ }^{13} \mathrm{C}\left\{{ }^{1} \mathrm{H}\right\}$ NMR $\left(75 \mathrm{MHz}, \mathrm{CDCl}_{3}, \mathrm{ppm}\right)$

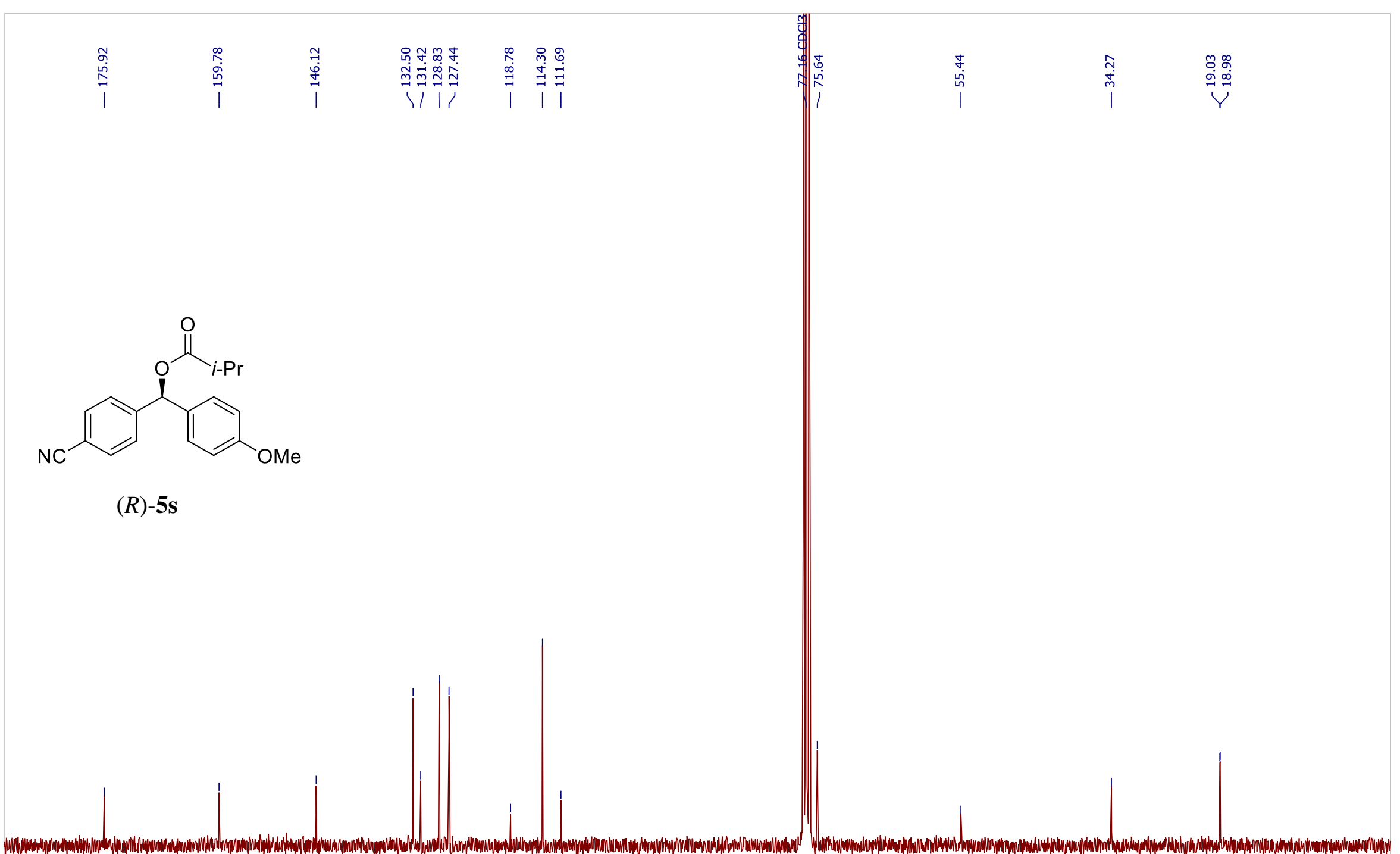

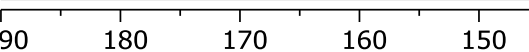
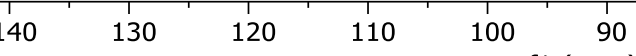

f1 (ppm) 
${ }^{1} \mathrm{H}$ NMR (300 MHz, $\left.\mathrm{CDCl}_{3}, \mathrm{ppm}\right)$

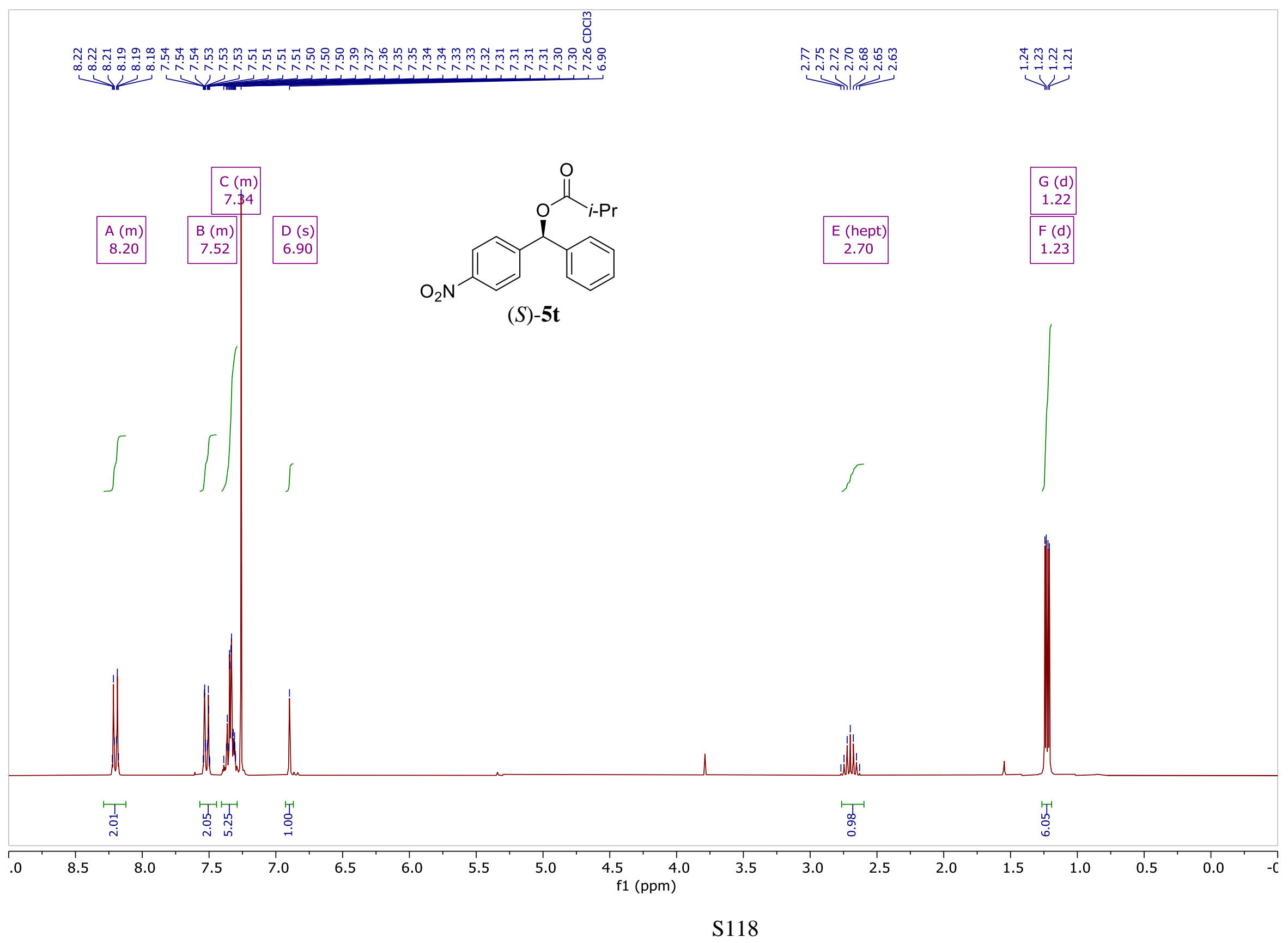


${ }^{13} \mathrm{C}\left\{{ }^{1} \mathrm{H}\right\}$ NMR $\left(75 \mathrm{MHz}, \mathrm{CDCl}_{3}, \mathrm{ppm}\right)$

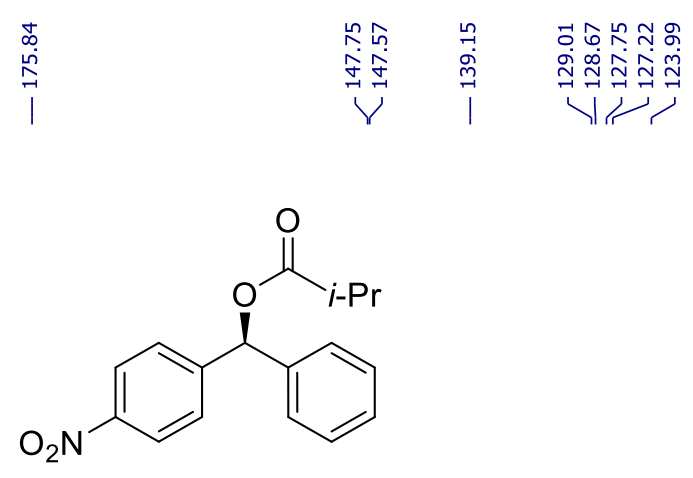

(S)-5t
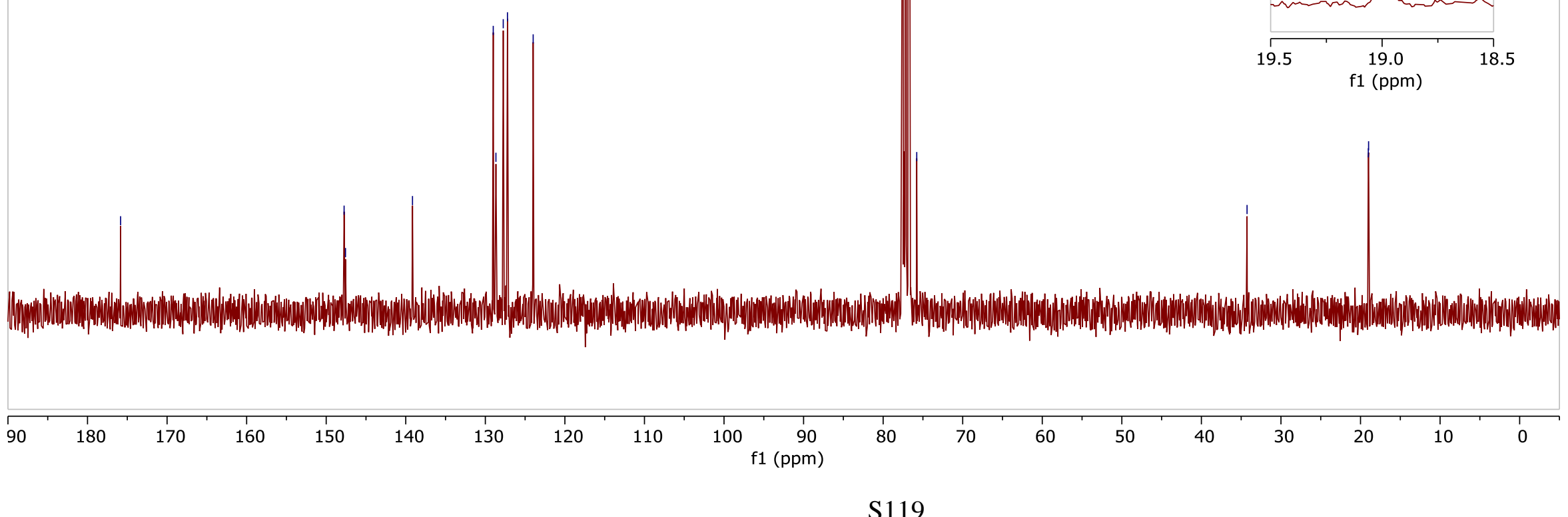
${ }^{1} \mathrm{H}$ NMR (300 MHz, $\left.\mathrm{CDCl}_{3}, \mathrm{ppm}\right)$

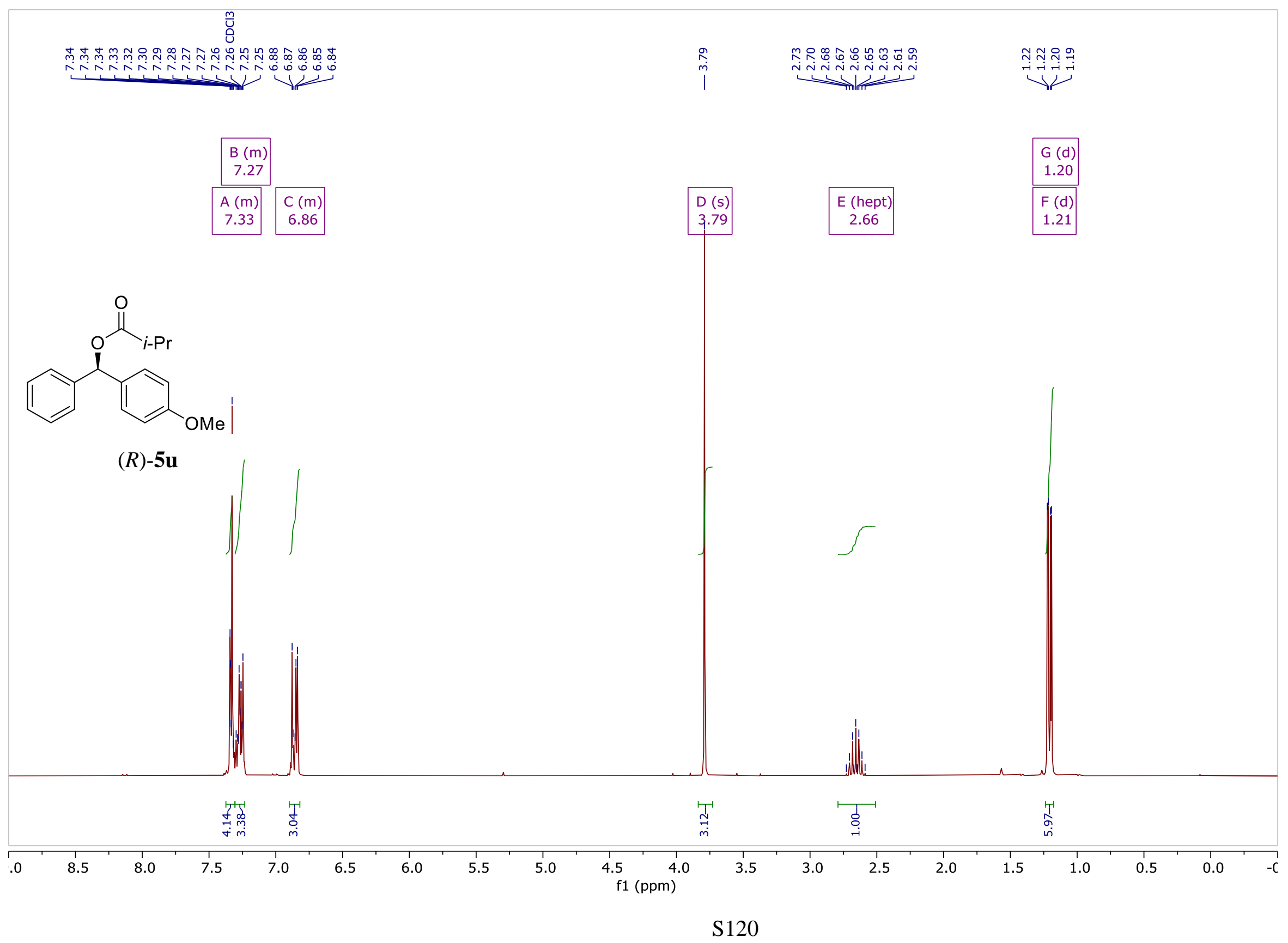


${ }^{1} \mathrm{H}$ NMR (300 MHz, $\left.\mathrm{CDCl}_{3}, \mathrm{ppm}\right)$




${ }^{13} \mathrm{C}\left\{{ }^{1} \mathrm{H}\right\}$ NMR $\left(75 \mathrm{MHz}, \mathrm{CDCl}_{3}, \mathrm{ppm}\right)$



